\title{
Mudança de Estrutura de Representação de Operadores em Morfologia Matemática
}

Ronaldo Fumio Hashimoto

TESE APRESENTADA

$\mathrm{AO}$

INSTITUTO DE MATEMÁTICA E ESTATÍSTICA

DA

UNIVERSIDADE DE SÃO PAULO

PARA

OBTENÇÃO DO GRAU DE DOUTOR

EM

MATEMÁTICA APLICADA

Área de Concentração: Ciência da Computação

Orientador: Prof. Dr. Junior Barrera

—São Paulo, julho de 2000— 


\section{Mudança de Estrutura de Representação de Operadores em Morfologia Matemática}

Este exemplar corresponde à redação final da tese devidamente corrigida e defendida por Ronaldo Fumio Hashimoto e aprovada pela comissão julgadora.

São Paulo, 11 de agosto de 2000.

Banca examinadora:

- Prof. Dr. Junior Barrera (orientador) - IME-USP

- Profa. Dra. Cristina Gomes Fernandes - IME-USP

- Prof. Dr. Gerald Jean Francis Banon - DPI-INPE

- Prof. Dr. Nelson Delfino d'Avila Mascarenhas - DC-UFSCar

- Prof. Dr. Roberto de Alencar Lotufo - DCA-UNICAMP 
"Depois de uma longa jornada, o viajante pára, às vezes, e volvendo os olhos para trás, contempla mais uma vez todo caminho andado, revivendo as emoções passadas. Isto lhe dá novo alento para prosseguir a caminhada corajosamente. Assemelhamo-nos a ele. Já percorremos um trecho do caminho. O mundo da 'ciência' já não é terra tão estranha para nós. Passo por passo, aprendemos a conhecê-la e amá-la melhor." 
Para Angélica e Beatriz 


\section{Agradecimentos}

Tenho certeza de que há muitas pessoas que contribuíram para a realização deste trabalho, e que não é possível enumerá-las todas aqui. Mas, gostaria de agradecer de uma forma muito especial:

Em primeiro lugar, gostaria de agradecer ao meu orientador, professor Junior Barrera, pela sugestão do problema, pela excelente orientação, pelas discussões, pelo apoio nos momentos difíceis, pelos "puxões de orelha" quando necessário, pela paciência e compreensão durante todo o doutorado.

Ao Prof. Serra, que me abriu as portas para passar quase um ano no "Centre de Morfologie Mathématique (CMM) de l'École Nationale Supérieure des Mines de Paris", em Fontainebleau. Foi um período muito enriquecedor, tanto no aspecto humano como profissional.

Ao Prof. Jeulin, pela orientação no trabalho de classificação de texturas de amostras de revestimento de paredes. Ao Antoine Aubert, pela prestativa ajuda e paciência durante o meu estágio no CMM.

Aos professores do Departamento de Ciência da Computação, que contribuíram para a minha formação acadêmica.

À minha esposa Angélica e à minha filha Beatriz, pela compreensão, pelo apoio e pela presença constante durante os momentos cruciais do meu doutorado.

Aos amigos de Fontainebleau, em especial modo às famílias Gérard e Brigitte, Flavio e Sylvie, que sempre se mostraram muito hospitaleiras. Não poderia deixar de citar também a família Pires que, em Fontainebleau, sempre apoiou a minha estadia por lá.

Ao José, que sempre me mostrou que todo trabalho é digno de ser bem feito.

À Mariana, pela demonstração de carinho e pela companhia durante todo este doutorado.

À Dona Mira, pela paciência nas aulas de francês.

A todos meus amigos, que souberam me apoiar durante a realização deste trabalho.

Ao $\mathrm{CNPq}$, pelo apoio financeiro para a realização do doutorado sanduíche na França.

E finalmente, aos membros da banca examinadora, pelas sugestões e contribuições que enriqueceram bastante esta tese.

A todos, meu muito obrigado! 


\section{Resumo}

A área de Visão Computacional tem como objetivo principal a extração de informação a partir de imagens digitais. Uma das técnicas mais promissoras para abordar este problema é a Morfologia Matemática.

O paradigma central da Morfologia Matemática é a decomposição de operadores entre reticulados completos em termos de um conjunto de operadores elementares da Morfologia Matemática, que pode ser descrito através de uma linguagem formal, a Linguagem Morfológica. Uma implementação da Linguagem Morfológica é chamada de Máquina Morfológica e um programa da Máquina Morfológica é uma implementação de um operador para esta máquina.

Assim, resolver um problema de Visão Computacional por Morfologia Matemática pode ser entendido como encontrar uma frase da Linguagem Morfológica (ou equivalentemente, um programa para uma Máquina Morfológica), que seja capaz de extrair a informação desejada.

Para uma frase da Linguagem Morfológica, pode existir um número infinito de outras frases (da Linguagem Morfológica) que são sinônimas, ou seja, diferentes frases podem expressar um mesmo operador.

Um teorema chave em Morfologia Matemática é o seguinte: qualquer operador entre reticulados completos pode ser decomposto em termos de um conjunto de operadores elementares da Morfologia Matemática. Este teorema foi provado pela apresentação de duas expressões canônicas de decomposição, chamadas de sup-decomposição e inf-decomposição, que têm uma estrutura puramente paralela.

Neste trabalho apresentamos resultados no sentido de, dada uma representação canônica de um operador entre reticulados que resolve um problema de Visão Computacional, encontrar uma frase da Linguagem Morfológica que envolva um número mínimo de operadores elementares. Este problema é extremamente complexo e o abordamos utilizando técnicas de Otimização Combinatória (como, por exemplo, método de "branch and bound" e estratégia gulosa).

Como a representação canônica tem uma estrutura puramente paralela e representações seqüenciais são usualmente mais eficientes em máquinas seqüenciais convencionais, apresentamos resultados no sentido de mudar a representação canônica para estruturas seqüenciais. 


\section{Abstract}

The main aim of Computer Vision problems is the extraction of information from digital images. A powerful technique to solve these problems is Mathematical Morphology.

A central paradigm in Mathematical Morphology is the decomposition of operators between Complete Lattices by a set of elementary operators of Mathematical Morphology. This paradigm can be formalized by the use of a formal language, called the Morphological Language. An implementation of the Morphological Language is called Morphological Machine and a program for a Morphological Machine is just an implementation of an operator for this machine.

Therefore, solving a problem of Computer Vision by Mathematical Morphology can be understood as finding a phrase of the Morphological Language (or equivalently, a program for a Morphological Machine) that can extract the desired information.

A phrase of the Morphological Language can have infinite synonymous phrases (of the Morphological Language), that is, different phrases can express the same operator.

A key theorem in Mathematical Morphology is the following: any operator between Complete Lattices can be decomposed by a set of elementary operators of Mathematical Morphology. This theorem was proved by showing two canonical structures called sup-decomposition and inf-decomposition. These canonical structures are strongly parallel.

In this work, we formalize and present results for the following problem: given an operator between Complete Lattices that solves a problem of Computer Vision, from its sup-decompositon (or inf-decompositon), find a synonymous phrase of the Morphological Language that has a minimal number of elementary operators. This problem is extremely complex and we study it by using Combinatorial Optimation techniques (for example, branch and bound method and greedy strategy).

Since the sup-decomposition and inf-decomposition of an operator have parallel structures and sequential representations are usually more efficient for computation in conventional sequential machines, we present techniques for transforming the canonical decompositions into purely sequential decompositions. 


\section{Sumário}

1 Introdução 1

1.1 Visão Computacional . . . . . . . . . . . . . . . . . . . . . 1

1.2 Morfologia Matemática . . . . . . . . . . . . . . . . 1

1.2.1 Decomposição Canônica de Operadores . . . . . . . . . . . . 2

1.2.2 Otimização da Estrutura de Decomposição . . . . . . . . . . . . . 3

1.3 Contribuição e Organização da Tese . . . . . . . . . . . . . . . . . . . . 3

2 Fundamentos Matemáticos $\quad 5$

2.1 Teoria dos Conjuntos . . . . . . . . . . . . . . . 5

2.1.1 Noções Fundamentais de Conjunto . . . . . . . . . . . . . . . 6

2.1.2 Conjunto dos Números Inteiros . . . . . . . . . . . . 7

2.1.3 Operações com Conjuntos . . . . . . . . . . . . . . . . . . 7

2.1.4 Produto Cartesiano . . . . . . . . . . . . . . . . . . . . 8

2.1.5 Relações sobre Conjuntos . . . . . . . . . . . . . . . . 9 9

2.1.6 Classes de Equivalência . . . . . . . . . . . . . . . . . . . 9

2.1.7 Partição de um Conjunto . . . . . . . . . . . . . . . . . . . . 10

2.1 .8 Funções . . . . . . . . . . . . . . . . . . 11

2.2 Teoria dos Grupos . . . . . . . . . . . . . . . . . . . 12

3 Morfologia Matemática $\quad 13$

3.1 Teoria dos Reticulados . . . . . . . . . . . . . . . . . . 13

3.1.1 Conjuntos Parcialmente Ordenados . . . . . . . . . . . . . . . 14

3.1.2 Elementos Notáveis de um Conjunto Parcialmente Ordenado . . . . 14 
3.1.3 Reticulados . . . . . . . . . . . . . . . . 15

3.2 Reticulado de Conjuntos $P(E) \ldots \ldots \ldots \ldots$

3.2 .1 Invariante de um Conjunto . . . . . . . . . . . . . . . 18

3.3 Reticulado dos Operadores . . . . . . . . . . . . . . . . . . . . 19

3.4 Outros Reticulados Estudados . . . . . . . . . . . . . . . . 20

3.4.1 Reticulado de Conjuntos $P(W) \ldots \ldots . \ldots . \ldots 21$

3.4.2 Reticulado de Coleção de Conjuntos $P(P(W))$. . . . . . . . . . 21

3.4.3 Reticulado de Coleção de Intervalos Maximais . . . . . . . . . . . . 21

3.4.4 Reticulado das Funções Binárias . . . . . . . . . . . . . . . . . . . 26

3.4.5 Reticulado dos $W$-Operadores . . . . . . . . . . . . . 26

3.5 Teorema da Decomposição de Operadores . . . . . . . . . . . . . . . 28

3.5.1 Operadores Elementares: Erosão e Dilatação . . . . . . . . . . . . . 28

3.5.2 Decomposição Canônica de $W$-Operadores . . . . . . . . . . . . . 29

3.5.3 Decomposição Minimal de $W$-Operadores . . . . . . . . . . . . 30

3.5.4 Propriedades da Base de um $W$-Operador . . . . . . . . . . . . 31

3.6 Decomposição de Operadores Especiais . . . . . . . . . . . . . . . . . 33

3.6.1 Operadores Crescentes Invariantes por Translação . . . . . . . . . . 33

3.6.2 Aberturas e Fechamentos Invariantes por Translação . . . . . . . . 34

3.7 Mudança de Estrutura de Representação . . . . . . . . . . . . . . . . . . 35

3.7.1 Mudança de Representação de Erosões e Dilatações . . . . . . . . . 35

3.8 Problemas Estudados nesta Tese . . . . . . . . . . . . . . . . . . . . . . 39

3.8.1 Decomposição de Elementos Estruturantes . . . . . . . . . . . . . . 39

3.8.2 Representação Compacta de W-Operadores . . . . . . . . . . . . . 40

3.8.3 Decomposição Seqüencial de $W$-Operadores . . . . . . . . . . . . . 40

4 Decomposição de Elementos Estruturantes $\quad 41$

4.1 Propriedades de uma Decomposição de um Elemento Estruturante . . . . . 41

4.2 Propriedades do Fecho Convexo . . . . . . . . . . . . . . . . . . . 44

4.3 Algumas Medidas Tomadas sobre um Elemento Estruturante . . . . . . . . 46

4.3.1 Lados ou Arestas de um Elemento Estruturante . . . . . . . . . . . 46

4.3.2 Vetor Projeção de um Elemento Estruturante . . . . . . . . . . . . 51 
4.3.3 Vetor Retangular de um Elemento Estruturante . . . . . . . . . . . 53

4.3.4 Limite Inferior de uma Decomposição . . . . . . . . . . . . . . . . . 53

4.4 Conclusão . . . . . . . . . . . . . . . . . . . . . . . . 53

5 Decomposição de Elementos Estruturantes Convexos $\quad 55$

5.1 Algoritmos Clássicos para Decompor Elementos Estruturantes Convexos . 55

5.2 Método Guloso . . . . . . . . . . . . . . . . . . . . . . . . 56

5.3 Decomposição Ótima de Elementos Estruturantes Convexos . . . . . . . . 56

5.3.1 Propriedades Necessárias para o Algoritmo . . . . . . . . . . . . . . 57

5.3.2 Apresentação do Algoritmo Proposto . . . . . . . . . . . . . . . . . 57

5.4 Resultados Experimentais . . . . . . . . . . . . . . . . . . 60

5.5 Corretude do Algoritmo . . . . . . . . . . . . . . . . . 62

5.5.1 Propriedades do Fecho Convexo para Elementos Estruturantes Convexos ......................... 62

5.5.2 A Saída do Algoritmo é uma Decomposição . . . . . . . . . . . . . 63

5.5.3 A Saída do Algoritmo é uma Decomposição Ótima . . . . . . . . . 68

5.6 Complexidade do Algoritmo . . . . . . . . . . . . . . . . . . . . . . 69

5.6.1 Complexidade dos Passos 05 e $06 \ldots \ldots$. . . . . . . . 70

5.6 .2 Complexidade dos Passos 08 e $12 \ldots \ldots$. . . . . . . . 71

5.6 .3 Complexidade do Algoritmo . . . . . . . . . . . . . . . . 72

5.7 Conclusão . . . . . . . . . . . . . . . . . . . 72

5.8 Apêndice do Capítulo $5 \ldots \ldots \ldots \ldots$. . . . . . . . . . . . . . . . . .

5.8.1 Prova da Proposição $5.15 \ldots$. . . . . . . . . . . . . . . . 73

5.8 .2 Prova da Proposição $5.16 \ldots \ldots$. . . . . . . . . . . 78

6 Decomposição de Elementos Estruturantes Simplesmente Conexos 85

6.1 O Algoritmo de Park e Chin . . . . . . . . . . . . . . . . . . 85

6.2 Famílias Infinitas de Elementos Estruturantes . . . . . . . . . . . . 87

6.2.1 Famílias de Elementos Estruturantes Simplesmente Conexos . . . . 88

6.2.2 Um Elemento em qualquer Decomposição não é Simplesmente Conexo 91

6.3 Complexidade do Algoritmo de Park e Chin . . . . . . . . . . . . . . . . 96

6.4 Conclusão . . . . . . . . . . . . . . . . . . . . . 96 
7 Decomposição de Elementos Estruturantes Arbitrários $\quad 97$

7.1 Introdução . . . . . . . . . . . . . . . . . . . . 97

7.1 .1 O Método "Branch and Bound" . . . . . . . . . . . . . . 98

7.1 .2 A Árvore de Decomposição . . . . . . . . . . . . . . . . . . . . . 98

7.1.3 Condições Necessárias para Nós Viáveis . . . . . . . . . . . . . . 100

7.2 Decomposição Ótima de um Elemento Estruturante . . . . . . . . . . . . . 103

7.3 Comparação com Alguns Algoritmos Conhecidos . . . . . . . . . . . . . . 107

7.3.1 Algoritmo de Zhuang e Haralick . . . . . . . . . . . . . . 107

$7.3 .2 \quad$ Algoritmo do Xu . . . . . . . . . . . . . . 107

7.3.3 Algoritmo de Park e Chin . . . . . . . . . . . . . . . . . . . 112

7.4 Resultados Experimentais . . . . . . . . . . . . . . . . . 112

7.4 .1 Discos Euclidianos . . . . . . . . . . . . . . . . 113

7.4.2 Elementos Estruturantes Convexos . . . . . . . . . . . . . . . 114

7.4.3 Elementos Estruturantes Decomponíveis, Conexos e não Conexos, com e sem Buracos . . . . . . . . . . . . . . . . . . 115

7.5 Conclusão . . . . . . . . . . . . . . . . . . . . 117

7.6 Apêndice do Capítulo $7 \ldots \ldots \ldots$. . . . . . . . . . . . . . . . . . . . . . .

7.6.1 Resultados Necessários para a Prova da Proposição 7.13 . . . . . 118

7.6 .2 Prova da Proposição 7.13 . . . . . . . . . . . . . . . . . . 120

8 Representação Compacta de $W$-Operadores 123

8.1 Representação Compacta . . . . . . . . . . . . . . . . . . . . . . . . . . 124

8.2 Representação Compacta para Operadores não Crescentes . . . . . . . . 126

8.3 Representação Compacta para Aberturas e Fechamentos Invariantes por Translação . . . . . . . . . . . . . . . . . . . . . . 128

8.4 Conversão da Sup-Representação para a Representação Compacta . . . . . 130

8.5 Conclusão . . . . . . . . . . . . . . . . . . . . . . 130

9 Sup-Decomposição para Decomposições Seqüenciais 133

9.1 Propriedades de Coleções de Intervalos Maximais . . . . . . . . . . . . . 133

9.2 Equação de Fatoração de Minkowski . . . . . . . . . . . . . . . . . . 135

9.2.1 Limite Superior para $W \ldots \ldots$. . . . . . . . . . . . 135 
9.2 .2 Limite Superior para $\mathbf{X}_{W} \ldots \ldots \ldots \ldots \ldots \ldots$

9.2 .3 Limites Inferiores para $\mathbf{X}_{W} \ldots \ldots \ldots \ldots \ldots \ldots \ldots$

9.3 Simplificação para Coleções de Extremidade Direita Fixa . . . . . . . . . 141

9.3.1 Simplificação do Limite Inferior . . . . . . . . . . . . . . . . 141

9.3.2 Conjuntos viáveis para $C \ldots \ldots \ldots$. . . . . . . . . 144

9.4 Conversão da Sup-Decomposição para Decomposições Seqüenciais … . 146

9.5 O Algoritmo Implementado para Mudança de Estrutura . . . . . . . 148

9.6 Exemplos de Aplicação do Algoritmo . . . . . . . . . . . . . . . . . . . 148

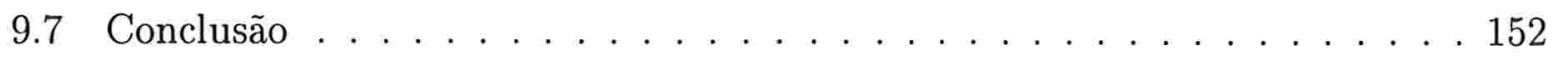

10 Conclusão Final 155

10.1 Conclusões e Contribuições . . . . . . . . . . . . . . . . . . . 155

10.1.1 Decomposição de Elementos Estruturantes Convexos … . . 156

10.1.2 Decomposição de Elementos Estruturantes Simplesmente Conexos . 156

10.1.3 Decomposição de Elementos Estruturantes Arbitrários . . . . . 157

10.1.4 Representação Compacta de $W$-Operadores . . . . . . . . 157

10.1.5 Decomposição Seqüencial de $W$-Operadores . . . . . . . . . . 157

10.2 Perspectivas Futuras . . . . . . . . . . . . . . . . . . . 158

A Classificação de Texturas a partir de Transformações Morfológicas 159

A.1 Introdução . . . . . . . . . . . . . . . . . . . . . . . . 159

A.2 Amostras e Aquisição de Imagens . . . . . . . . . . . . . . . 160

A.3 Medidas Morfológicas . . . . . . . . . . . . . . . . . . . . . . . . 161

A.3.1 A Capacidade de Choquet . . . . . . . . . . . . . . . 161

A.3.2 Medidas Morfológicas . . . . . . . . . . . . . . . . . . . 162

A.4 Introdução à Análise de Correspondências . . . . . . . . . . . . . . . . 165

A.5 Resultados Experimentais . . . . . . . . . . . . . . . . . . 166

A.5.1 Primeira Etapa: Análise das Medidas . . . . . . . . . . . 167

A.5.2 Segunda Etapa: Redução do Número de Variáveis . . . . . . . . 167

A.5.3 Estudo Final . . . . . . . . . . . . . . . . . 167

A.6 Conclusão . . . . . . . . . . . . . . . . . . . . . . . . 168 
A.7 Agradecimentos . . . . . . . . . . . . . . . . . . . 168

B Artigos Publicados e Submetidos a Revistas e Congressos 


\section{Lista de Figuras}

3.1 (a) Um conjunto $A \in \mathcal{P}(E)$. (b) Alguns invariantes de $A$. . . . . . . . . . . . . . 17

3.2 Diagramas que representam algumas coleções de um reticulado completo Booleano. . . 22

3.3 Isomorfismo de reticulado entre $\Pi_{W}$ and $\Pi_{W^{\prime} / W} \ldots \ldots \ldots \ldots$. . . . . . . . . . . . 24

3.4 Os quatro isomorfismos de reticulados. . . . . . . . . . . . . . . . . . . 28

3.5 (a) Os conjuntos $X$ e $B .(b)$ Dilatação de $X$ por $B \ldots \ldots \ldots \ldots$

$3.6 \quad$ (a) Um EE $A$. (b) O fecho convexo de $A \ldots \ldots \ldots$

4.1 Um ponto $y$ e os eixos $\vec{u}_{0}, \vec{u}_{1}, \vec{u}_{2}$ e $\vec{u}_{3} \ldots \ldots \ldots \ldots \ldots$

4.2 Um EE $A$ com os eixos $\vec{u}_{0}, \vec{u}_{1}, \vec{u}_{2}$ e $\vec{u}_{3} \ldots \ldots \ldots \ldots \ldots$

4.3 Ilustração para a demonstração do Lema 4.12 . . . . . . . . . . . . . . . . . . . . 48

4.4 Os oito lados de um EE convexo A . . . . . . . . . . . . . . . . . . . . . . . 48

4.5 Os lados $E_{i}(A)$ e $E_{i+1}(A)$ de um EE $A$. . . . . . . . . . . . . . . . . . . . . 49

4.6 Extremidades do lado $E_{i}(A) \ldots \ldots \ldots \ldots$

4.7 (a) Um EE $A$. (b) O fecho convexo de $A$. (c) O código de cadeia de $C(A)$. (d) $\mathrm{O}$ vetor

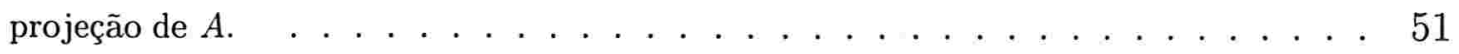

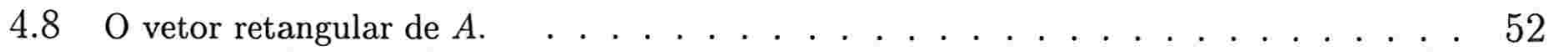

5.1 (a) Um EE $A$. (b) Elementos de $\mathcal{B}(A)$. (c) Saída do algoritmo DecCon. (d) Saída do algoritmo do Xu. . . . . . . . . . . . . . . . 59

5.2 Aplicação do algoritmo DeCCon para um exemplo simples. . . . . . . . . . . . . 60

5.3 Elementos de $\mathcal{R} . \quad \ldots \ldots \ldots \ldots \ldots \ldots \ldots$

$5.4 \quad$ Elementos de $\mathcal{S} \ldots \ldots \ldots \ldots \ldots \ldots \ldots \ldots$

5.5 Todos elementos de $\mathcal{R}_{1}$ são decomponíveis. . . . . . . . . . . . . . 76

5.6 Todos elementos de $\mathcal{S}_{1}$ são decomponíveis. $\ldots \ldots \ldots \ldots$

5.7 Todas possibilidades de $C\left(B_{i}\right) \oplus C\left(B_{k}\right)$ se $C\left(B_{i}\right) \in \mathcal{R}_{2}$ e $C\left(B_{k}\right) \in \mathcal{S}_{2} . \quad \ldots \ldots$. . . 76 
6.1 (a) Um EE $A \quad$ (b) Uma decomposição de $A . \quad \ldots \ldots$. . . . . . . . . . . . . . . . . 86

6.2 Os eixos Cartesianos $\vec{v}_{0}$ e $\vec{v}_{1} \ldots \ldots \ldots \ldots \ldots$

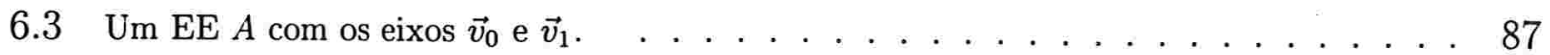

$6.4(a)-(e)$ Os EE's $D_{1}, D_{2}, D_{3}, D_{4}$ e $D_{5} \ldots \ldots \ldots \ldots \ldots \ldots$

$6.5(a)-(c)$ EE's $X_{1}, Y_{1}$ e $Z_{1} \ldots \ldots \ldots \ldots \ldots \ldots \ldots$

$6.6(a)-(d)$ As quatro possibilidades para $X$ e $Y \ldots \ldots \ldots \ldots$

$6.7(a)-(b)$ EE's $i D_{1}$ e $i D_{2} \ldots \ldots \ldots \ldots \ldots \ldots$

$6.8(a) X_{i+1}=\left(X_{i}\right)_{d_{1}} \cup\left(D_{3}\right)_{p_{i}}(b) Y_{i+1}=\left(Y_{i}\right)_{d_{1}} \cup\left(D_{4}\right)_{q_{i}}$ e $(c) Z_{i+1}=\left(Z_{i}\right)_{d_{1}} \cup\left(D_{5}\right)_{q_{i}} . \quad$. . 91

$6.9(a)-(c)$ Os EE's $X_{6}, Y_{7}$ e $Z_{7}$ e suas respectivas decomposições. . . . . . . . . . 95

7.1 Um nó $Y$ na árvore de decomposição. . . . . . . . . . . . . . . . . . . . 99

7.2 (a) Um EE $A$. (b) Seqüência de invariantes de $A$. (c) Árvore de decomposição de $A . \quad$. 99

$7.3(a)-(b)$ Um EE $A$ e $C(A)$. (c) Seqüência de invariantes de $A .(d)-(e) Z$ e $C(Z)$. . . 100

7.4 Aplicação do algoritmo para um exemplo simples. . . . . . . . . . . . . 106

7.5 (a) Um EE convexo. (b) Um EE conexo, decomponível, que contém um buraco. (c) Um EE conexo, decomponível, que não contém buracos. (d) Um EE não conexo, decomponível, que contém alguns buracos. (e) Um EE não conexo, decomponível, que não contém buracos. . . . . . . . . . . . . . . . . . 113

$7.6(a)-(c)$ Discos euclidianos de raios 2,3 e $4 \ldots \ldots \ldots 114$

9.1 Limite superior para $\mathbf{X}_{W} \ldots \ldots \ldots \ldots \ldots$

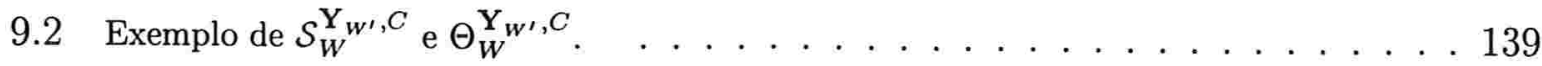

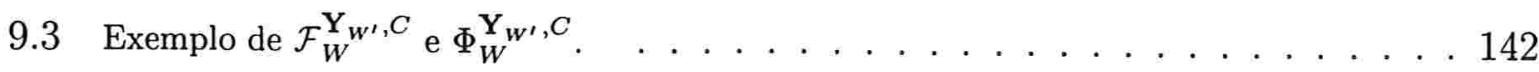

9.4 Limite superior e as soluções para o exemplo apresentado na Figura 9.3. . . . . . . . 143

9.5 Um diagrama da árvore de representação. . . . . . . . . . . . . . . . . . . 147

9.6 Applicação do método proposto para um exemplo simples. . . . . . . . . . . . . . . 149

9.7 Alguns exemplos de transformação da sup-decomposição para decomposições seqüenciais. . . . . . . . . . . . . . . . . . . 152

A.1 (a) Qualidade $Q 1$ ("too early"), (b) Qualidade $Q 2$ ("normal"), (c) Qualidade $Q 3$ ("too late"). . . . . . . . . . . . . . . . . . . . . 160

A.2 Exemplo de uma curva de fechamento (lado esquerdo) e uma curva de abertura (lado direito) por elementos estruturantes quadrados. 
A.3 Exemplo de uma curva de erosão (lado direito) e uma curva de dilatação (lado esquerdo) por segmentos verticais. . . . . . . . . . . . . . . . . . . 164

A.4 Exemplo de "watersheds" . . . . . . . . . . . . . . . . . . . . . . . . 164

A.5 Exemplo da distribuição de picos (parte esquerda) e de vales (parte direita) . . . . . . 165

A.6 Pontuação da classificação para as curvas de erosão e dilatação pelo quadrado e a correspondente representação das variáveis no primeiro plano de fatores. . . . . . . . . . 169

A.7 Pontuação da classificação para as curvas de erosão e dilatação por linhas verticais e a correspondente representação das variáveis no primeiro plano de fatores. . . . . . . 170 


\section{Lista de Tabelas}

5.1 Tempo médio para encontrar uma decomposição ótima e a média do número de pontos nesta decomposição. . . . . . . . . . . . . . . . . . . . . . 6 61

7.1 Discos decomponíveis. . . . . . . . . . . . . . . . . . . . . . . . . . . . . . 114

7.2 Discos que não são decomponíveis. . . . . . . . . . . . . . . . . . . . 115

7.3 Tempo médio para se detectar a solução ótima de EE's convexos. . . . . . . . . . 115

7.4 Tempo para se detectar a solução ótima de alguns EE's convexos. . . . . . . . . . 116

7.5 Tempo médio para encontrar uma decomposição ótima de EE's convexos. . . . . . . 116

7.6 Tempo médio para detectar a primeira solução de alguns EE's. . . . . . . . . . 117

7.7 Tempo para detectar a primeira solução e a solução ótima de alguns EE's. . . . . . . 117

7.8 Tempo para detectar a primeira solução de alguns EE's. . . . . . . . . . . . . . . 118

9.1 Tempo utilizado pelo algoritmo para encontrar todas representações seqüenciais. . . . 153 



\section{Capítulo 1}

\section{Introdução}

\subsection{Visão Computacional}

A área de Visão Computacional tem como objetivo principal a extração de informação a partir de imagens digitais. Historicamente, este problema vem sendo abordado por um conjunto de técnicas de naturezas diversas: filtragem linear, reconhecimento de padrões, teoria da informação, modelagem estatística, etc. $[5,16,31]$. Dentre essas técnicas, uma das mais promissoras é a Morfologia Matemática (MM) [41, 42].

\subsection{Morfologia Matemática}

A MM, fundada por J. Serra e G. Matheron nos anos sessenta, tornou-se uma disciplina muito importante na área de Visão Computacional. Seu domínio de atuação cobre um largo espectro de aplicações em Processamento de Imagens que vai desde o tratamento de imagens microscópicas até o processamento de imagens de satélites, passando por aplicações industriais, médicas, automação de escritório, etc. [41, 42].

O paradigma central da MM é a decomposição de operadores entre reticulados completos em termos de um conjunto de operadores elementares da MM, que pode ser descrito através de uma linguagem formal, a Linguagem Morfológica (LM) [9]. Uma implementação da LM é chamada de Máquina Morfológica (MaqM) [8, 9] e um programa da MaqM é uma implementação de um operador para esta máquina.

Assim, resolver um problema de Visão Computacional por MM pode ser entendido como encontrar uma frase da LM (ou equivalentemente, um programa para uma MaqM), que seja capaz de extrair a informação desejada.

Classicamente, a tarefa de criar programas para MaqM's é realizada empiricamente, baseada na experiência do analista no uso de operadores da MM. Embora esta metodologia 
tenha trazido muitos resultados práticos, que evidenciam a potencialidade da $\mathrm{MM}$, ela restringe o universo de usuários capazes de criarem novos programas para MaqM's aos especialistas em MM.

Para permitir que especialistas em áreas de aplicação (médicos, geólogos, astrônomos, etc.), que não têm grande conhecimento em MM, possam independentemente programar MaqM's, começam a ser pesquisadas técnicas para geração automática de programas para MaqM's $[10,11]$. O princípio básico dessas técnicas consiste em:

(1) descrever os operadores em estruturas de representação de conhecimento de alto nível de abstração;

(2) traduzir automaticamente a descrição de alto nível dos operadores em uma frase da LM;

(3) Buscar uma representação econômica da frase da LM estimada no Passo (2), ou seja, uma outra frase equivalente de tal forma que o número de operações executadas pela MaqM seja menor possível.

A teoria apresentada neste trabalho é útil para efetuar o Passo (3). Para um estudo mais aprofundado dos Passos (1) e (2) veja os trabalhos [10,11, 12].

Para uma frase da LM, podem existir um número infinito de outras frases (da LM) que são sinônimas, ou seja, diferentes frases podem expressar um mesmo operador [12]. Dessa forma, estamos interessados em encontrar uma estrutura de representação otimizada do operador estimado, no sentido de envolver operadores elementares de tal forma que o número de operações executadas pela MaqM seja mínimo. No caso geral, este problema é de complexidade exponencial.

\subsubsection{Decomposição Canônica de Operadores}

Um teorema chave em MM é o seguinte: qualquer operador entre reticulados completos pode ser decomposto em termos de um conjunto de operadores elementares da MM. Este teorema foi provado pela apresentação de duas expressões canônicas de decomposição, que têm uma estrutura puramente paralela $[6,7]$.

Este resultado garante a completude da LM para expressar operadores entre reticulados completos, isto é, ele garante que qualquer operador entre reticulados completos pode ser programável em uma MaqM.

Por outro lado, as formas canônicas de representação nem sempre constituem a estrutura de decomposição mais adequada para representar operadores. De fato, são conhecidos muitos exemplos de decomposição de operadores particulares, com estruturas seqüenciais de operadores elementares de modo que o número de operações executadas pela MaqM é consideravelmente menor $[7,6]$. 


\subsubsection{Otimização da Estrutura de Decomposição}

Os operadores elementares usados na decomposição canônica são caracterizados por uma coleção de intervalos fechados, conhecida como base do operador [12]. Barrera e Salas [12] estudaram operações sobre intervalos fechados para geração automática de frases da LM. Estas operações podem ser utilizadas para construir a base de um operador a partir de uma representação desse operador por uma frase da LM. Uma aplicação deste estudo é verificar se frases da LM são sinônimas, uma vez que qualquer representação pode ser reduzida à base que determina unicamente o operador [12]. Agora, o problema inverso, ou seja, a transformação da representação canônica em outras estruturas de representação que usam operadores elementares de forma que o número de operações na MaqM seja mínimo, no caso geral, é extremamente complexo, mas tem um papel importante em programação automática de MaqM's.

Dois operadores elementares da MM são a dilatação e a erosão. Esses operadores, quando invariantes por translação, são caracterizados por um elemento estruturante, e em algumas MaqM's a eficiência de um programa depende fortemente do tamanho desses elementos estruturantes. Desta forma, muitos pesquisadores têm estudado a decomposição de um elemento estruturante por somas de Minkowski e proposto diferentes algoritmos. $\mathrm{Xu}$ [49] desenvolveu um algoritmo para encontrar uma decomposição ótima de elementos estruturantes convexos e Park e Chin [35] propuseram um algoritmo para elementos estruturantes 8-conexos e sem "buracos". Estudamos o problema de decompor elementos estruturantes e propomos um algoritmo guloso para decompor elementos estruturantes convexos e um outro algoritmo que usa a estratégia de "branch and bound" para decompor elementos estruturantes arbitrários.

\subsection{Contribuição e Organização da Tese}

O problema geral de mudança da representação canônica em outras estruturas de representação que usam operadores elementares de forma que o número de operações na MaqM seja mínimo foi pouco explorado até o presente momento. Na literatura encontramos poucas referências a respeito deste problema. Dessa forma, a principal contribuição deste trabalho é fazer um estudo detalhado e bem acabado deste problema e abrir, ou indicar, caminhos para pesquisas futuras nesta área ainda pouco explorada.

Nesta tese, os capítulos estão organizados da seguinte forma. Seguindo a introdução, neste capítulo, o Capítulo 2 introduz a base matemática para a compreensão da pesquisa desenvolvida nesta tese. No Capítulo 3, descrevemos alguns aspectos do estado da arte em MM no que se refere à decomposição de operadores entre reticulados completos e à transformação de estruturas de decomposição. No Capítulo 4, apresentamos os fundamentos matemáticos para se obter uma decomposição de um elemento estruturante e que servem de apoio para os Capítulos 5, 6 e 7. No Capítulo 5 apresentamos um algoritmo guloso para decompor elementos estruturantes convexos. No Capítulo 6, mostramos que o algoritmo 
de Park e Chin [35] é limitado, pois ele não é capaz de decompor três famílias infinitas de elementos estruturantes decomponíveis simplesmente conexos. No Capítulo 7, apresentamos um algoritmo que usa uma estratégia de "branch and bound" para decompor elementos estruturantes arbitrários. No Capítulo 8, apresentamos uma nova representação mais compacta (ou seja, que utiliza um número menor de operadores elementares) que a decomposição na forma canônica. No Capítulo 9, formalizamos e apresentamos um algoritmo para transformar uma decomposição canônica de um operador (que tem uma estrutura puramente paralela) em representações puramente seqüenciais do mesmo operador (quando elas existem). Finalmente, no Capítulo 10, concluimos e discutimos algumas possíveis direções para pesquisas futuras.

Esta tese possui ainda dois apêndices. No Apêndice A mostramos um exemplo de aplicação de transformações morfológicas para classificação de texturas de revestimento de paredes. E no Apêndice B, apresentamos as publicações decorridas deste trabalho.

A partir do Capítulo 4, todos resultados são contribuições originais desta tese. 


\section{Capítulo 2}

\section{Fundamentos Matemáticos}

Neste capítulo, introduziremos a base matemática necessária para a compreensão da pesquisa desenvolvida nesta tese. Na Seção 2.1, recordaremos algumas definições e propriedades da teoria de conjuntos. Na Seção 2.2, veremos algumas definições da teoria dos grupos.

Assumiremos aqui que o leitor tenha alguma familialidade com conceitos básicos da Teoria da Complexidade Computacional, razão pela qual não incluímos nenhuma seção sobre tais conceitos. Ademais, a terminologia utilizada segue exatamente a de Garey e Johnson [21].

As definições e conceitos que apresentamos neste capítulo não pretendem abranger toda a teoria, mas apenas introduzir os elementos necessários para a compreensão do texto nos capítulos seguintes. Desta forma, somente algumas propriedades serão demonstradas; as provas das propriedades e dos resultados mencionados neste capítulo que possam ser encontradas nas referências citadas não serão apresentadas.

O conteúdo deste capítulo pode ser facilmente compreendido e não é nenhum obstáculo para a leitura desta tese, pois em sua maioria as definições e propriedades são bastante conhecidas na literatura. No entanto, aconselhamos uma rápida leitura do mesmo com o objetivo de se familiarizar com as notações e nomenclaturas utilizadas nos capítulos posteriores.

\subsection{Teoria dos Conjuntos}

Nesta seção, recordaremos algumas propriedades e alguns conceitos básicos da teoria dos conjuntos [17] que serão utilizados ao longo desta tese. Na Subseção 2.1.1, apresentamos as noções fundamentais de conjunto. Na Subseção 2.1.2, apresentamos a notação utilizada nesta tese para o conjunto dos números inteiros. Na Subseção 2.1.3, definimos algumas operações com conjuntos e mostramos algumas de suas propriedades. Na Subseção 2.1.4, 
definimos o produto cartesiano entre dois ou mais conjuntos. Na Subseção 2.1.5, definimos alguns tipos de relações entre conjuntos. Na Subseção 2.1.6, definimos o que são as classes de equivalência de um conjunto e apresentamos algumas de suas propriedades. Na Subseção 2.1.7, definimos o que é uma partição de um conjunto. Finalmente, na Subseção 2.1.8, definimos o que vem a ser uma função.

\subsubsection{Noções Fundamentais de Conjunto}

Um conjunto $A$ é uma coleção de elementos de um determinado universo, denotado genericamente por $U$. Se um elemento $x$ do universo $U$ pertence ao conjunto $A$, então escrevemos $x \in A$ (leia-se, $x$ pertence a $A$ ); caso contrário, $x \notin A$.

Um conjunto finito é um conjunto com um número finito de elementos. Um conjunto que não é finito diz-se infinito. Salvo indicação contrária, usaremos o termo "conjunto" significando "conjunto finito". A cardinalidade de um conjunto finito $A$, denotada por $|A|$, é o número de elementos pertencentes a $A$.

Um conjunto vazio, denotado por $\emptyset$, é um conjunto que não contém nenhum elemento. Um conjunto unitário é um conjunto constituído por um único elemento.

Um conjunto $A$ está contido em um outro conjunto $B$ se, e somente se, todo elemento de $A$ pertence a $B$, isto é, $A \subseteq B \Leftrightarrow(x \in A \Rightarrow x \in B)$. Neste caso, diz-se que $A$ é um subconjunto de $B$.

Dizemos que um conjunto $A$ é um superconjunto de um outro conjunto $B$ se, e somente se, $B$ é um subconjunto de $A$, isto é, $A \supseteq B \Leftrightarrow B \subseteq A$.

Um conjunto $A$ está propriamente contido em um outro conjunto $B$ se, e somente se, $A$ está contido em $B$ e $A$ é diferente de $B$, isto é, $A \subset B \Leftrightarrow(A \subseteq B$ e $A \neq B)$. Neste caso, diz-se que $A$ é um subconjunto próprio de $B$.

Dizemos que um conjunto $A$ é igual a um outro conjunto $B$ se, e somente se, $A \subseteq B$ e $B \subseteq A$.

A proposição seguinte é um consequência imediata da relação de inclusão entre conjuntos.

Proposição 2.1 Sejam $A$ e $B$ dois conjuntos. Se $A \subseteq B$ e $A \neq B$, então $|A|<|B|$.

O conjunto das partes de um conjunto $E$, denotado por $\mathcal{P}(E)$, é o conjunto cujos elementos são todos os subconjuntos de $E$, isto é, $\mathcal{P}(E)=\{X: X \subseteq E\}$. Note que $\emptyset \in \mathcal{P}(E), E \in \mathcal{P}(E)$ e se $|E|=n$, então $|\mathcal{P}(E)|=2^{n}$. 


\subsubsection{Conjunto dos Números Inteiros}

Ao longo deste texto, denotaremos o conjunto dos números inteiros por $\mathbb{Z}$. Dizemos que um número inteiro $x$ é não negativo se, e somente se, $x \geq 0$. O conjunto de todos os inteiros não negativos é denotado por $\mathbb{Z}_{+}$. Um número inteiro $x$ é dito positivo se, e somente se, $x>0$. Denotaremos o conjunto dos números inteiros positivos por $\mathbb{Z}_{+}^{*}$.

\subsubsection{Operações com Conjuntos}

Nesta subseção apresentaremos três operações básicas com conjuntos: complemento, interseç̧ão e união.

Sejam $A$ e $E$ dois conjuntos tais que $A \subseteq E$. O complemento de $A$ em relação a $E$ é o conjunto de todos os elementos de $E$ que não pertencem a $A$, isto é, $A_{E}^{c}=\{x \in E: x \notin A\}$. Quando o conjunto $E$ está claramente definido pelo contexto, escreveremos simplesmente $A^{c}$.

A seguinte proposição [17, p. 48] apresenta duas propriedades da operação complemento.

Proposição 2.2 Sejam $A, B$ e E três conjuntos tais que $A, B \subseteq E$. Então valem as seguintes propriedades do complemento em relação a $E$.

(a) $\left(A^{c}\right)^{c}=A$;

(b) $A \subseteq B \Rightarrow B^{c} \subseteq A^{c}$.

A intersecção de dois conjuntos $A$ e $B$ é o conjunto de todos os elementos que pertencem simultanemente a $A$ e a $B$, isto é, $A \cap B=\{x: x \in A$ e $x \in B\}$.

A interseç̧ão de $n$ conjuntos $A_{1}, A_{2}, \cdots, A_{n}$ é o conjunto de todos elementos que pertencem simultaneamente a todos estes $n$ conjuntos, isto é, $\cap_{i=1}^{n} A_{i}=\left\{x: x \in A_{1}\right.$ e $x \in$ $A_{2}$ e $\cdots$ e $\left.x \in A_{n}\right\}$.

Dizemos que dois conjuntos $A$ e $B$ são disjuntos se, e somente se, $A \cap B=\emptyset$.

A união de dois conjuntos $A$ e $B$ é o conjunto de todos os elementos que pertencem a $A$ ou a $B$, isto é, $A \cup B=\{x: x \in A$ ou $x \in B\}$.

A união de $n$ conjuntos $A_{1}, A_{2}, \cdots, A_{n}$ é o conjunto de todos elementos que pertencem a pelo menos um destes $n$ conjuntos, isto é, $\cup_{i=1}^{n} A_{i}=\left\{x: x \in A_{1}\right.$ ou $x \in A_{2}$ ou $\cdots$ ou $x \in$ $\left.A_{n}\right\}$.

A seguir apresentamos algumas propriedades da intersecção e da união entre conjuntos. As demonstrações destas propriedades podem ser encontradas em [17].

Proposição 2.3 Seja $U$ o conjunto universo. Se $A, B, C \subseteq U$, então valem as seguintes propriedades. 

(a) $A \cap B \subseteq A$ e $A \cap B \subseteq B$
(b) $A \subseteq A \cup B$ e $B \subseteq A \cup B$
(c) $A \subseteq B \Leftrightarrow A \cap B=A$
(d) $A \subseteq B \Leftrightarrow A \cup B=B$
(e) $A \subseteq B \Rightarrow A \cap C \subseteq B \cap C$
(f) $A \subseteq B \Rightarrow A \cup C \subseteq B \cup C$
(g) $A \subseteq B$ e $A \subseteq C \Rightarrow A \subseteq B \cap C$
(i) $A \subseteq C$ e $B \subseteq C \Rightarrow A \cap B \subseteq C$
(h) $A \subseteq B$ e $A \subseteq C \Rightarrow A \subseteq B \cup C$
(k) $A \cap B=B \cap A$
(j) $A \subseteq C$ e $B \subseteq C \Rightarrow A \cup B \subseteq C$
(m) $(A \cap B) \cap C=A \cap(B \cap C)$
(l) $A \cup B=B \cup A$
(o) $A \cap(B \cup C)=(A \cap B) \cup(A \cap C)$
(q) $x \in A \cap B \Leftrightarrow x \in A$ e $x \in B$
(n) $(A \cup B) \cup C=A \cup(B \cup C)$
(p) $A \cup(B \cap C)=(A \cup B) \cap(A \cup C)$
(r) $x \in A \cup B \Leftrightarrow x \in A$ ou $x \in B$

Os dois resultados seguintes (Proposições 2.4 e 2.5) são conseqüências das propriedades distributivas da intersecção e união dadas nas Proposições 2.3.o e 2.3.p.

Proposição 2.4 Sejam $A, X, Y \in \mathcal{P}(E)$ tais que se $A \subseteq X \subseteq Y$, então $\left(A \cup X_{Y}^{c}\right) \cap X=A$.

Prova:

$$
\begin{aligned}
\left(A \cup X^{c}\right) \cap X & =(A \cap X) \cup\left(X^{c} \cap X\right) & & \text { (pela Proposição 2.3.o) } \\
& =A & & \text { (uma vez que } A \subseteq X \text { ). }
\end{aligned}
$$

Proposição 2.5 Sejam $A, X, Y \in \mathcal{P}(E)$ tais que $X \subseteq Y$ e $A \subseteq Y$. Se $A-X=X_{Y}^{c}$, então $(A \cap X) \cup X_{Y}^{c}=A$.

Prova: Primeiramente, provaremos que $A-X=X_{Y}^{c} \Leftrightarrow X_{Y}^{c} \subseteq A$ da seguinte forma.

$$
\begin{aligned}
A-X=X_{Y}^{c} & \Leftrightarrow A \cap X_{Y}^{c}=X_{Y}^{c} \\
& \Leftrightarrow X_{Y}^{c} \subseteq A .
\end{aligned}
$$

Finalmente, $(A \cap X) \cup X_{Y}^{c}=A$, uma vez que

$$
\begin{aligned}
(A \cap X) \cup X_{Y}^{c} & =\left(A \cup X_{Y}^{c}\right) \cap\left(X \cup X_{Y}^{c}\right) & & \text { (pela Proposição 2.3.p) } \\
& =A \cap Y & & \text { (uma vez que } X_{Y}^{c} \subseteq A \text { ) } \\
& =A & & \text { (uma vez que } A \subseteq Y \text { ). }
\end{aligned}
$$

\subsubsection{Produto Cartesiano}

Sejam $A$ e $B$ dois conjuntos. Dados $x \in A$ e $y \in B$, dizemos que $(x, y)$ é, ou forma, um par ordenado. Dizemos que o elemento $x$ é a primeira coordenada e que o elemento $y$ é a segunda coordenada do par ordenado $(x, y)$. 
Generalizando, dados $n$ conjuntos $A_{1}, A_{2}, \cdots, A_{n}$ e elementos $x_{i} \in A_{i}$, para $i \in$ $\{1,2, \cdots, n\}$, dizemos que $\left(x_{1}, x_{2}, \cdots, x_{n}\right)$ é, ou forma, uma $n$-upla ordenada. Cada elemento $x_{i}, i \in\{1,2, \cdots, n\}$, é a $i$-ésima coordenada da $n$-upla $\left(x_{1}, x_{2}, \cdots, x_{n}\right)$.

Sejam $A$ e $B$ dois conjuntos. O produto cartesiano de $A$ por $B$, denotado por $A \times B$, é o conjunto formado por todos pares ordenados $(x, y)$, tais que $x \in A$ e $y \in B$, isto é, $A \times B=\{(x, y): x \in A, y \in B\}$. O quadrado de um conjunto $A$ é o produto cartesiano de $A$ por $A$, isto é, $A^{2}=A \times A$.

Generalizando, o produto cartesiano de $n$ conjuntos $A_{1}, A_{2}, \cdots, A_{n}$, denotado por $A_{1} \times$ $A_{2} \times \cdots \times A_{n}$, é o conjunto formado por todas $n$-uplas ordenadas $\left(x_{1}, x_{2}, \cdots, x_{n}\right)$, onde $x_{i} \in A_{i}, i \in\{1,2, \cdots, n\}$, isto é, $A_{1} \times A_{2} \times \cdots \times A_{n}=\left\{\left(x_{1}, x_{2}, \cdots, x_{n}\right): x_{1} \in A_{1}, x_{2} \in\right.$ $\left.A_{2}, \cdots, \dot{x}_{n} \in A_{n}\right\}$. No caso particular em que $A=A_{1}=A_{2}=\cdots=A_{n}, A^{n}=$ $A_{1} \times A_{2} \times \cdots \times A_{n}$ é a $n$-ésima potência de $A$.

\subsubsection{Relações sobre Conjuntos}

Uma relação binária $R$ de um conjunto $A$ com um conjunto $B$ é uma terna ordenada $R=(G, A, B)$, onde $G \subseteq A \times B$. Dizemos que $x$ está relacionado com $y$ pela relação $R$ se, e somente se, $(x, y) \in G$, isto é, $x R y \Leftrightarrow(x, y) \in G$.

Quando $B=A$, dizemos simplesmente que $R$ é uma relação sobre $A$.

A inversa de uma relação binária $R$ de um conjunto $A$ com um conjunto $B$ é a relação binária $R^{-1}$ de $B$ com $A$ definida como, para todo $x \in A$ e $y \in B, y R^{-1} x \Leftrightarrow x R y$.

Uma relação binária $R$ sobre um conjunto $A$ é dita reflexiva se, e somente se, para todo $x \in A$, temos que $x R x$.

Uma relação binária $R$ sobre um conjunto $A$ é dita simétrica se, e somente se, para todo $x, y \in A$, temos que $x R y \Rightarrow y R x$.

Uma relação binária $R$ sobre um conjunto $A$ é dita anti-simétrica se, e somente se, para todo $x, y \in A$, temos que $x R y$ e $y R x \Rightarrow x=y$.

Uma relação binária $R$ sobre um conjunto $A$ é dita transitiva se, e somente se, para todo $x, y, z \in A$, temos que $x R y$ e $y R z \Rightarrow x R z$.

Uma relação binária $R$ sobre um conjunto $A$ é uma relação de equivalência se, e somente se, ela é reflexiva, simétrica e transitiva.

\subsubsection{Classes de Equivalência}

Sejam $A$ um conjunto e $R$ uma relação de equivalência sobre $A$. Se $x, y \in A$ são tais que $x R y$, então dizemos que $x$ é equivalente a $y$ segundo $R$ e denotaremos $x \equiv_{R} y$. Quando a relação de equivalência está claramente definida pelo contexto, escreveremos simplesmente $x \equiv y$. 
Seja $A$ um conjunto não vazio. Sejam $R$ uma relação de equivalência sobre $A$ e $x \in A$. A classe de equivalência segundo $R$ do elemento $x$ é o conjunto de todos os elementos de $A$ que são equivalentes a $x$ segundo $R$, isto é, $\mathcal{C}_{R}(x)=\left\{y \in A: y \equiv_{R} x\right\}$. Quando a relação de equivalência está claramente definida pelo contexto, escreveremos simplesmente $\mathcal{C}(x)$.

Note que, para todo $x \in A, x \in \mathcal{C}_{R}(x)$, pois pela propriedade reflexiva de $R, x R x$. Logo, $\mathcal{C}_{R}(x)$ nunca é vazia, isto é, $\mathcal{C}_{R}(x) \neq \emptyset$.

Seja $A$ um conjunto não vazio. Sejam $R$ uma relação de equivalência sobre $A$ e $x \in A$. Dizemos que a classe de equivalência $\mathcal{C}_{R}(x)$ é determinada ou definida pelo elemento $x$, o qual por sua vez, é o representante de $\mathcal{C}_{R}(x)$.

A seguinte proposição [17, p. 163] é uma propriedade de classes de equivalência.

Proposição 2.6 Seja $R$ uma relação de equivalência em um conjunto não vazio $A$ e sejam $x, y \in A$. Então valem as seguintes propriedades:

(a) $\mathcal{C}_{R}(x)=\mathcal{C}_{R}(y) \Leftrightarrow x \equiv_{R} y$

(a) $\operatorname{se} \mathcal{C}_{R}(x) \cap \mathcal{C}_{R}(y) \neq \emptyset$, então $\mathcal{C}_{R}(x)=\mathcal{C}_{R}(y)$

Seja $R$ uma relação de equivalência em um conjunto não vazio $A$ e sejam $x, y \in A$. Pela Proposição 2.6a, temos que se $y \in \mathcal{C}_{R}(x)$, então $\mathcal{C}_{R}(x)=\mathcal{C}_{R}(y)$. Portanto, qualquer elemento de $\mathcal{C}_{R}(x)$ é um representante de $\mathcal{C}_{R}(x)$.

Sejam $A$ um conjunto não vazio e $R$ uma relação de equivalência sobre $A$. O conjunto quociente de $A$ pela relação de equivalência $R$ é o conjunto formado por todas as classes de equivalência segundo $R$, isto é, $\mathcal{Q}_{R}(A)=\left\{\mathcal{C}_{R}(x): x \in A\right\}$. Quando a relação de equivalência está claramente definida pelo contexto, escreveremos simplesmente $\mathcal{Q}(A)$.

Denotaremos por $\mathcal{R}_{R}(A)$, ou simplesmente $\mathcal{R}(A)$ quando a relação de equivalência está claramente definida pelo contexto, o conjunto formado por exatamente um representante de cada classe de equivalência de $\mathcal{Q}_{R}(A)$, isto é, $\mathcal{R}_{R}(A)$ é um conjunto tal que $\left|\mathcal{R}_{R}(A)\right|=$ $\left|\mathcal{Q}_{R}(A)\right|$.

\subsubsection{Partição de um Conjunto}

Seja $A$ um conjunto não vazio. Uma partição de $A$ é um conjunto $P(A)$ de partes não vazias de $A$, disjuntas duas a duas e cuja união é $A$, isto é, $P(A) \subseteq \mathcal{P}(A)$ que satisfaz as três seguintes condições:

(a) se $X \in P(A)$, então $X \neq \emptyset$;

(b) se $X_{i}, X_{j} \in P(A)$ e $X_{i} \neq X_{j}$, então $X_{i} \cap X_{j}=\emptyset$;

(c) $\cup\{X: X \in P(A)\}=A$.

A seguinte proposição [17, p. 165] afirma que uma relação de equivalência sobre um conjunto $A$ define uma partição de $A$. 
Proposição 2.7 Dados um conjunto não vazio $A$ e uma relação de equivalência $R$ em $A$, o conjunto quociente $\mathcal{Q}_{R}(A)$ é uma partição de $A$.

\subsubsection{Funções}

Uma função (ou mapeamento ou transformação) de um conjunto $A$ em um outro conjunto $B$ é uma relação binária $f=(F, A, B)$ de $A$ com $B$ que satisfaz as seguintes condições:

(1) $\forall x \in A, \exists y \in B:(x, y) \in F$;

(2) $\forall x \in A$, se $(x, y),(x, z) \in F$, então $y=z$;

Uma função $f$ de $A$ em $B$ é indicada por $f: A \rightarrow B$. Para cada elemento $x \in A$, o único elemento $y \in B$ tal que $(x, y) \in F$ é chamado imagem de $x$ pela função $f$ ou valor da função $f$ em $x$ e é denotado por $y=f(x)$.

Dada uma função $f: A \rightarrow B$, os conjuntos $A$ e $B$ são chamados de domínio e contradomínio da função $f$. O conjunto $\{f(x): x \in A\} \subseteq B$ é chamado imagem da função $f$ e é denotado por $f(A)$.

Uma função $f: A \rightarrow B$ é sobrejetora se, e somente se, para todo $y \in B$, existe um $x \in A$ tal que $f(x)=y$, isto é, $\forall y \in B, \exists x \in A: f(x)=y$.

Uma função $f: A \rightarrow B$ é injetora se, e somente se, para quaisquer dois elementos distintos $x_{1}, x_{2} \in A$, temos imagens distintas $f\left(x_{1}\right), f\left(x_{2}\right) \in B$, isto é, se $x_{1}, x_{2} \in A$ e $x_{1} \neq x_{2}$, então $f\left(x_{1}\right) \neq f\left(x_{2}\right)$.

Uma função $f: A \rightarrow B$ é bijetora se, e somente se, ela é sobrejetora e injetora ao mesmo tempo.

Dada uma função $f: A \rightarrow B$, a relação inversa $f^{-1}$ de $B \operatorname{com} A$ (isto é, $f^{-1}=$ $\left(F^{-1}, B, A\right)$ onde $\left.F^{-1}=\{(y, x):(x, y) \in F\}\right)$, é uma função se, e somente se, $f$ é uma função bijetora. A função $f^{-1}$, se existe, é chamada função inversa de $f$.

Sejam $f: A \rightarrow B$ e $g: B \rightarrow C$. Definimos a composição da função $f$ com a função $g$ a função $g \cdot f: A \rightarrow C \operatorname{como} g \cdot f(x)=g(f(x))$, para todo $x \in A$. A função $g \cdot f$ é também chamada de função composta de $g$ e $f$. Em geral denotamos $g \cdot f$ simplesmente por $g f$ ou $g(f)$.

A seguir, definimos uma função bastante conhecida e muito utilizada nesta tese. A função $\lceil\cdot\rceil$, definida do conjunto dos números reais no conjunto dos números inteiros, é chamada de função teto e ela é definida da seguinte forma: se $x$ é um número real qualquer, então $\lceil x\rceil$ corresponde ao menor inteiro maior ou igual a $x$. Por exemplo, se $y=2.7182$, então $\lceil x\rceil=3$ e $\lceil-x\rceil=-2$. 


\subsection{Teoria dos Grupos}

Um conjunto $G$, munido com uma operação binária + é um grupo, se satisfaz os seguintes axiomas:

(a) $a+(b+c)=(a+b)+c, \quad \forall a, b, c \in G$

(b) $G$ contém um elemento $e$ tal que $e+a=a+e=a$, para todo $a \in G$;

(c) $\forall a \in G$, existe um elemento em $G$, denotado por $-a$, tal que $a+(-a)=$ $(-a)+a=e$.

Um grupo $G$ munido com uma operação binária + será denotado por $(G,+)$. O elemento $e$ que satisfaz a condição $(b)$ é chamado de elemento neutro de $(G,+)$.

Um grupo $(G,+)$ é chamado de grupo abeliano se, e somente se, para quaisquer $a, b \in$ $G, a+b=b+a$. 


\section{Capítulo 3}

\section{Morfologia Matemática}

A MM, iniciada por J. Serra e G. Matheron nos anos sessenta, na "École Nationale Superiéure de Mines de Paris", em Fontainebleau, tornou-se uma disciplina muito importante em Visão Computacional. Seu domínio de atuação cobre um largo espectro de aplicações em que vai desde o tratamento de imagens microscópicas até o processamento de imagens de satélites, passando por aplicações industriais, médicas, automação de escritório, etc. [41, 42]. Um exemplo de aplicação da MM para classificação de texturas de revestimento de paredes pode ser visto no Apêndice A.

O objetivo deste capítulo é descrever alguns aspectos do estado da arte em MM. Na Seção 3.1, recordaremos alguns conceitos e propriedades de teoria dos reticulados. Como os mapeamentos estudados nesta tese estão definidos de $\mathcal{P}(E)$ em $\mathcal{P}(E)$, apresentamos na Seção 3.2 o reticulado de subconjuntos $(\mathcal{P}(E), \subseteq)$ e algumas de suas propriedades. $\mathrm{Na}$ Seção 3.3 definiremos os operadores estudados nesta tese. Além disso, apresentamos algumas definições e propriedades desses operadores. Na Seção 3.4 apresentamos quatro reticulados completos Booleanos estudados nesta tese. Veremos que estes reticulados são isomorfos dois a dois. Na Seção 3.5, abordaremos o estado da arte em MM no que se refere à decomposição de mapeamentos entre reticulados completos e, na Seção 3.6, no que se refere à decomposição de algumas classes de operadores especiais (crescentes e invariantes por translação, etc...). Finalmente, na Seção 3.7, apresentamos o estado da arte no que se refere à transformação de estruturas de representação dos $W$-operadores.

Para o leitor interessado somente nos resultados de decomposição de elementos estruturantes, é suficiente ler somente as Seções 3.2 e 3.7 (Subseção 3.7.1).

\subsection{Teoria dos Reticulados}

Nesta seção, recordaremos alguns conceitos e propriedades de teoria dos reticulados [14, 24] que serão utilizados ao longo desta tese. 


\subsubsection{Conjuntos Parcialmente Ordenados}

Uma relação binária $\leq$ sobre um conjunto não vazio $A$ é uma relação de ordem se, e somente se, ela é reflexiva, anti-simétrica e transitiva.

Exemplo 3.1 Dado um conjunto $E$, a relação de inclusão $\subseteq$ no conjunto $\mathcal{P}(E)$ é uma relação de ordem.

Um conjunto $A$ munido com uma relação de ordem $\leq$ é um conjunto parcialmente ordenado ou, resumidamente, um poset (do inglês, "partial ordered set") e ele é denotado por $(A, \leq)$, ou simplesmente por $A$ quando a relação de ordem está claramente definida pelo contexto.

Exemplo 3.2 Se $E$ é um conjunto tal que $|E|>1$, então $(\mathcal{P}(E), \subseteq)$ é um conjunto parcialmente ordenado.

\subsubsection{Elementos Notáveis de um Conjunto Parcialmente Orde- nado}

\subsubsection{Limitantes Superiores e Inferiores}

Seja $(A, \leq)$ um poset. Sejam $\ell, u \in A$ e $X \subseteq A$. Dizemos que $u$ é um limitante superior de $X$, se para todo $x \in X$ temos que $x \leq u$. Dizemos que $\ell$ é um limitante inferior de $X$ se para todo $x \in X$, temos que $\ell \leq x$. Se $X$ tem ambos um limitante superior e um limitante inferior, então $X$ é chamado um subconjunto limitado de $A$.

\subsubsection{Maior e Menor Elementos}

Sejam $(A, \leq)$ um poset e $X \subseteq A$. Um elemento $a \in X$ é chamado menor elemento de $X$ se $a \leq x$ para todo $x \in X$. Um elemento $b \in X$ é chamado maior elemento de $X$ se $x \leq b$ para todo $x \in X$.

Exemplo 3.3 O menor e o maior elemento do poset $(\mathcal{P}(E), \subseteq)$ são, respectivamente, $\emptyset$ e $E$, uma vez que, para todo elemento $X \in \mathcal{P}(E)$, verifica-se que $\emptyset \subseteq X \subseteq E$.

\subsubsection{Supremo e Ínfimo}

Sejam $A$ um poset e $X \subseteq A$. Se $X$ tem um limitante superior $u \in A$, o menor elemento do conjunto de limitantes superiores de $X$, se existe, é chamado de supremo de $X$ em $A$ e é denotado por $\vee X$. Similarmente, se $X$ tem um limitante inferior $\ell \in A$, o maior elemento 
do conjunto dos limitantes inferiores de $X$, se existe, é chamado de ínfimo de $X$ em $A$ e é denotado por $\wedge X$.

Exemplo 3.4 O infimo e o supremo de um subconjunto $\mathcal{X} \subseteq \mathcal{P}(E)$ são, respectivamente, $\cap\{X: X \in \mathcal{X}\}$ e $\cup\{X: X \in \mathcal{X}\}$.

Note que o supremo e o ínfimo de $X$ podem ou não pertencer a $X$. Se supremo pertence a $X$, então ele é o maior elemento de $X$. Similarmente, se o ínfimo pertence a $X$, então ele é o menor elemento de $X$.

\subsubsection{Reticulados}

Um conjunto parcialmente ordenado $(L, \leq)$ é um reticulado se, e somente se, qualquer conjunto de dois elementos $\{x, y\} \subseteq L$ possui um ínfimo e um supremo, denotados, respectivamente, por $x \wedge y$ e $x \vee y$.

Um sub-reticulado de um reticulado $L$ é um conjunto $A \subseteq L$ tal que, para todo $a, b \in A$, $a \wedge b \in A$ e $a \vee b \in A$.

Um reticulado $L$ é um reticulado completo se, e somente se, qualquer subconjunto de $L$ possui um ínfimo e um supremo em $L$. Qualquer reticulado finito é um reticulado completo.

Exemplo $3.5 O$ poset $(\mathcal{P}(E), \subseteq)$ é um reticulado completo, pois qualquer subconjunto $\mathcal{X} \subseteq \mathcal{P}(E)$ tem um infimo e um supremo dados, respectivamente, por $\wedge \mathcal{X}=\cap\{X: X \in$ $\mathcal{X}\}$ e $\vee \mathcal{X}=\cup\{X: X \in \mathcal{X}\}$.

Um reticulado $L$ é um reticulado distributivo se, e somente se, para todo $x, y, z \in L$, $x \wedge(y \vee z)=(x \wedge y) \vee(x \wedge z)$.

Dado um reticulado $L$ com o menor e maior elementos $\mathcal{O}$ e $\mathcal{I}$, respectivamente. Se $x, y \in L$ são tais que $x \wedge y=\mathcal{O}$ e $x \vee y=\mathcal{I}$, então $y$ é chamado complemento de $x$ e denotamos $y=x^{c}$. O reticulado $L$ é chamado de um reticulado complementado se, e somente se, qualquer elemento de $L$ tem um complemento.

Um reticulado $L$ é um reticulado Booleano se, e somente se, ele é distributivo e complementado.

Exemplo 3.6 O poset $(\mathcal{P}(E), \subseteq)$ é um reticulado Booleano, pois ele é distributivo (isto é, para quaisquer $X, Y, Z \in \mathcal{P}(E), X \cap(Y \cup Z)=(X \cup Y) \cap(X \cup Z))$ e complementado (isto é, para todo $X \in \mathcal{P}(E), X \cap X^{c}=\emptyset$ e $X \cup X^{c}=E$ ).

Teorema 3.7 (Lei de De Morgan) Seja $L$ um reticulado Booleano. Se $a, b \in L$, então $(a \wedge b)^{c}=a^{c} \vee b^{c} e(a \vee b)^{c}=a^{c} \wedge b^{c}$. 


\subsubsection{Princípio da Dualidade}

Seja $(L, \leq)$ um poset. Seja $\geq$ a relação inversa de $\leq$ sobre $L$ (isto é, $x \geq y \Leftrightarrow y \leq x$ ). Diremos que $(L, \geq)$, ou simplesmente $\tilde{L}$, é o poset dual de $(L, \leq)$.

Princípio da Dualidade [24, p. 19]: Se $(L, \leq)$ é um poset, então para toda definição, propriedade, afirmação, etc., referindo-se ao poset $(L, \leq)$ existe uma correspondente dual referindo-se ao poset $(L, \geq)$.

Por exemplo, se para um reticulado $(L, \leq)$ vale a propriedade: $a, b \in L \Rightarrow a \wedge b \leq$ $a \vee b$, então pelo princípio da dualidade, temos a seguinte propriedade dual válida para $o$ reticulado dual de $L: a, b \in \tilde{L} \Rightarrow a \vee b \geq a \wedge b$.

\subsubsection{Isomorfismo de Reticulados}

Sejam $\left(A, \leq_{A}\right)$ e $\left(B, \leq_{B}\right)$ dois reticulados. Seja $f: A \rightarrow B$ uma função bijetora. Dizemos que $f$ é um isomorfismo de reticulado de $A$ em $B$ se, e somente se, para todo $x, y \in A$, temos que $x \leq_{A} y \Leftrightarrow f(x) \leq_{B} f(y)$. Dizemos que $f$ é um dual-isomorfismo se, e somente se, para todo $x, y \in A$, temos que $x \leq_{A} y \Leftrightarrow f(y) \leq_{B} f(x)$.

Dois reticulados $A$ e $B$ são ditos isomorfos (respectivamente, dual-isomorfos) se, e somente se, existe um isomorfismo (respectivamente, dual-isomorfismo) entre eles.

\subsection{Reticulado de Conjuntos $\boldsymbol{P}(\boldsymbol{E})$}

Seja $E$ um conjunto não vazio. Vimos nos Exemplos 3.5 e 3.6 que o poset $(\mathcal{P}(E), \subseteq)$ é um reticulado completo Booleano. Nesta seção veremos algumas definições e propriedades deste reticulado.

Seja $E$ um conjunto não vazio que é um grupo Abeliano com respeito à operação binária denotada por + . O elemento neutro (que é também chamado de origem) de $(E,+)$ é denotado por $o$.

O transposto de um conjunto $X \in \mathcal{P}(E)$ (em relação à origem) é o subconjunto $X^{t} \in$ $\mathcal{P}(E)$ dado por $X^{t}=\{x \in E:-x \in X\}$.

Para todo $X \in \mathcal{P}(E)$ e $h \in E, X_{h}$ denota a translação de $X$ por $h$, isto é,

$$
X_{h}=\{x \in E: x-h \in X\} \text {. }
$$

Sejam $X, Y \in \mathcal{P}(E)$. A adição e subtração de Minkowski de $X$ e $Y$ são, respectivamente, os conjuntos $X \oplus Y \in \mathcal{P}(E)$ e $X \ominus Y \in \mathcal{P}(E)$ dados por $X \oplus Y=\cup\left\{X_{y}: y \in Y\right\}$ e $X \ominus Y=\cap\left\{X_{-y}: y \in Y\right\}$.

Na proposição [24, p. 82] que se segue, apresentamos algumas propriedades da adição de Minkowski. 


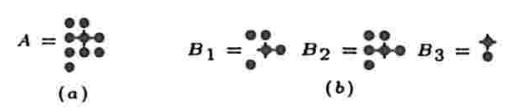

Figura 3.1: (a) Um conjunto $A \in \mathcal{P}(E)$. (b) Alguns invariantes de $A$.

Proposição 3.8 Sejam $X, Y, Z \in \mathcal{P}(E)$ e $h \in E$. Então,

(a) $X \oplus Y=\{x+y \in E: x \in X, y \in Y\}$ [24, p. 81, Eq. 4.20],

(b) $X \oplus\{h\}=X_{h}$ (efeito de translação),

(c) $X_{h} \oplus Y=X \oplus Y_{h}=(X \oplus Y)_{h}$,

(d) $Y \subseteq Z \Rightarrow X \oplus Y \subseteq X \oplus Z$,

(e) $X \oplus(Y \cup Z)=(X \oplus Y) \cup(X \oplus Z)$,

(f) $(X \oplus Y) \oplus Z=X \oplus(Y \oplus Z)$ (associatividade),

(g) $X \subseteq Y \Rightarrow X \oplus Z \subseteq Y \oplus Z$,

(h) $X \oplus Y=Y \oplus X$ (comutatividade).

Na próxima proposição [24, p. 82], apresentamos algumas propriedades da subtração de Minkowski.

Proposição 3.9 Sejam $X, Y, Z \in \mathcal{P}(E)$ e $h \in E$. Então,
(a) $X \ominus Y=\left\{h \in E: Y_{h} \subseteq X\right\}$ [24, p. 81, Eq. 4.22],
(b) $X \ominus\{h\}=X_{-h}$ (efeito de translação),
(c) $X_{h} \ominus Y=X \ominus Y_{-h}=(X \ominus Y)_{h}$,
(d) $Y \subseteq Z \Rightarrow X \ominus Y \supseteq X \ominus Z$,
(e) $X \ominus(Y \cup Z)=(X \ominus Y) \cap(X \ominus Z)$,
(f) $(X \ominus Y) \ominus Z=Y \ominus(X \oplus Z)$,
(g) $X \subseteq Y \Rightarrow X \ominus Z \subseteq Y \ominus Z$.

Como uma conseqüência da Proposição 3.8.c, temos o seguinte resultado.

Proposição 3.10 Se $X, Y \in \mathcal{P}(E)$, então, para todo $h \in E, X \oplus Y=X_{-h} \oplus Y_{h}$.

Prova: Seja $Z=X \oplus Y$. Assim,

$$
\begin{aligned}
X \oplus Y=Z & \Leftrightarrow(X \oplus Y)_{h}=Z_{h} & & \\
& \Leftrightarrow X \oplus Y_{h}=Z_{h} & & \text { (pela Proposição 3.8.c) } \\
& \Leftrightarrow\left(X \oplus Y_{h}\right)_{-h}=Z & & \\
& \Leftrightarrow X_{-h} \oplus Y_{h}=Z & & \text { (pela Proposição 3.8.c). }
\end{aligned}
$$

A adição e subtração de Minkowski satisfazem a seguinte propriedade [24, p. 84], conhecida como relação de adjunção.

Proposição 3.11 Se $X, Y, Z \in \mathcal{P}(E)$, então $X \oplus Y \subseteq Z \Leftrightarrow X \subseteq Z \ominus Y$. 


\subsubsection{Invariante de um Conjunto}

Sejam $X, Y \in \mathcal{P}(E)$. Dizemos que $Y$ é um conjunto invariante, ou simplesmente, um invariante de $X$ se, e somente se, $X=(X \ominus Y) \oplus Y$. Por exemplo, os conjuntos $B_{1}, B_{2}, B_{3}$, apresentados na Figura 3.1.b, são invariantes do conjunto $A$ apresentado na Figura 3.1.a.

Sejam $X, Y \in \mathcal{P}(E)$. Dizemos que $Y$ é um aberto de $X$ se, e somente se, $Y$ é um invariante de $X$.

Os resultados seguintes (Proposições 3.12 e 3.13) fornecem algumas propriedades de uma subtração de Minkowski seguida de uma adição de Minkowski pelo mesmo elemento estruturante. A Proposição 3.12 [24, p. 89, Eq. 4.56] é uma conseqüência imediata da Proposição 3.9.a e da definição de adição de Minkowski. A Proposição 3.13 é uma conseqüência direta da Proposição 3.11, substituindo $X$ por $Z \ominus Y$.

Proposição 3.12 Se $X, Y \in \mathcal{P}(E)$, então $(X \ominus Y) \oplus Y=\cup\left\{Y_{h}: h \in E\right.$ e $\left.Y_{h} \subseteq X\right\}$.

Proposição 3.13 Se $Z, Y \in \mathcal{P}(E)$, então $(Z \ominus Y) \oplus Y \subseteq Z$.

As Proposições 3.14 e 3.15 mostram algumas propriedades de invariantes de um dado conjunto em $\mathcal{P}(E)$. A primeira foi mostrada por Serra [41, p. 53] e a segunda por Zhuang e Haralick [51, Prop. 5].

Proposição 3.14 Sejam $A, X \in \mathcal{P}(E)$. Então, $X$ é um invariante de $A$ se, e somente se, existe $Y \in \mathcal{P}(E)$ tal que $A=Y \oplus X$.

Proposição 3.15 Sejam $A, X, Y \in \mathcal{P}(E)$. Se $A=X \oplus Y$, então $X$ e $Y$ são ambos invariantes de $A$.

Os dois próximos resultados (Proposições 3.16 e 3.17) relacionam invariança com translação. A Proposição 3.16 é uma conseqüência imediata das Proposições 3.10, 3.14 e 3.15; enquanto que a Proposição 3.17 é uma conseqüência da Proposição 3.8.c.

Proposição 3.16 Sejam $X, Y \in \mathcal{P}(E)$. Se $Y$ é um invariante de $X$, então, para todo $h \in E, Y_{h}$ é também um invariante de $X$.

Proposição 3.17 Sejam $X, Y \in \mathcal{P}(E)$ e $h \in E$. Então, $Y$ é invariante de $X$ se, $e$ somente se, $Y$ é invariante de $X_{h}$.

\section{Prova:}




$$
\begin{aligned}
X_{h} & =((X \ominus Y) \oplus Y)_{h} & & \text { (uma vez que } Y \text { é invariante de } X) \\
& =(X \ominus Y)_{h} \oplus Y & & \text { (pela Proposição 3.8.c) } \\
& =\left(X_{h} \ominus Y\right) \oplus Y & & \text { (pela Proposição 3.8.c). }
\end{aligned}
$$

Portanto $Y$ é um invariante de $X_{h}$.

$(\Leftarrow)$

$$
\begin{aligned}
X & =\left(X_{h}\right)_{-h} \\
& =\left(\left(X_{h} \ominus Y\right) \oplus Y\right)_{-h} \\
& =\left((X \ominus Y)_{h} \oplus Y\right)_{-h} \\
& =((X \ominus Y) \oplus Y)_{h-h} \\
& =(X \ominus Y) \oplus Y .
\end{aligned}
$$

Portanto $Y$ é um invariante de $X$.

\subsection{Reticulado dos Operadores}

Um mapeamento de $\mathcal{P}(E)$ em $\mathcal{P}(E)$ é chamado um operador. Os operadores serão denotados por letras gregas minúsculas $\alpha, \beta, \gamma, \ldots \mathrm{O}$ conjunto de todos operadores será denotado por $\Psi$ (letra grega maiúscula). O conjunto $\Psi$ herda a estrutura de reticulado completo Booleano do conjunto $(\mathcal{P}(E), \subseteq)$ quando definimos a relação de ordem $\leq$ sobre os elementos de $\Psi$ como, para quaisquer $\psi_{1}, \psi_{2} \in \Psi$,

$$
\psi_{1} \leq \psi_{2} \quad \Leftrightarrow \quad \psi_{1}(X) \subseteq \psi_{2}(X) \quad(X \in \mathcal{P}(E)) .
$$

O supremo e ínfimo de dois operadores $\psi_{1}$ e $\psi_{2}$ de $\Psi$ verificam, respectivamente, $\left(\psi_{1} \vee \psi_{2}\right)(X)=\psi_{1}(X) \cup \psi_{2}(X)$ e $\left(\psi_{1} \wedge \psi_{2}\right)(X)=\psi_{1}(X) \cap \psi_{2}(X)$, para todo $X \in \mathcal{P}(E)$.

O operador $\nu \in \Psi$ definido por $\nu(X)=X^{c}$, para todo $X \in \mathcal{P}(E)$, é chamado operador de negação.

O operador $\iota \in \Psi$ definido por $\iota(X)=X$, para todo $X \in \mathcal{P}(E)$, é chamado operador identidade. Observe que $\nu \nu=\iota$.

O operador dual de um operador $\psi$, denotado $\psi^{*}$, é dado por $\psi^{*}=\nu \psi \nu$. Observamos que $\left(\psi^{*}\right)^{*}=\psi$, uma vez que $\nu \nu=\iota$.

Um operator $\psi \in \Psi$ é chamado crescente se, e somente se, para todo $X, Y \in \mathcal{P}(E)$, $X \subseteq Y \Rightarrow \psi(X) \subseteq \psi(Y)$.

Um operator $\psi \in \Psi$ é chamado extensivo (respectivamente, anti-extensivo) se, e somente se, $\psi \leq \iota$ (respectivamente, $\iota \leq \psi)$.

Um operator $\psi \in \Psi$ é chamado idempotente se, e somente se, $\psi \psi=\psi$.

A proposição seguinte é uma conseqüência imediata da definição de operador dual.

Proposição 3.18 Seja $\psi \in \Psi$. 
(a) $\psi$ é crescente se, e somente se, $\psi^{*}$ é crescente;

(b) $\psi$ é idempotente se, e somente se, $\psi^{*}$ é idempotente;

(c) $\psi$ é extensivo se, e somente se, $\psi^{*} e ́$ anti-extensivo.

Prova: Prova de $(a)$ : Sejam $X, Y \in \mathcal{P}(E)$.

$(\Rightarrow)$

$$
\begin{aligned}
X \subseteq Y & \Leftrightarrow \nu(Y) \subseteq \nu(X) & & \text { (pela Proposição 2.2.b) } \\
& \Rightarrow \psi(\nu(Y)) \subseteq \psi(\nu(X)) & & \text { (uma vez que } \psi \text { é crescente) } \\
& \Leftrightarrow \nu(\psi(\nu(X))) \subseteq \nu(\psi(\nu(Y))) & & \text { (pela Proposição 2.2.b) } \\
& \Leftrightarrow(\nu \psi \nu)(X) \subseteq(\nu \psi \nu)(Y) & & \\
& \Leftrightarrow \psi^{*}(X) \subseteq \psi^{*}(Y) . & &
\end{aligned}
$$

\section{$(\Leftarrow)$}

Provamos que se $\psi$ é crescente, então $\psi^{*}$ também é crescente. Agora, considere que $\varphi=\psi^{*}$ é crescente. Assim, pela prova anterior, temos que $\varphi^{*}=\left(\psi^{*}\right)^{*}=\psi$ é crescente.

Prova de (b):

$$
\begin{aligned}
\psi \psi=\psi & \Leftrightarrow \nu \psi \psi \nu=\nu \psi \nu \\
& \Leftrightarrow \nu \psi \nu \nu \psi \nu=\nu \psi \nu \quad \text { (uma vez que } \nu \nu=\iota) \\
& \Leftrightarrow(\nu \psi \nu)(\nu \psi \nu)=\nu \psi \nu \\
& \Leftrightarrow \psi^{*} \psi^{*}=\psi^{*} .
\end{aligned}
$$

Prova de $(c)$ :

$$
\begin{aligned}
\iota \leq \psi & \Leftrightarrow \iota \nu \leq \psi \nu \\
& \Leftrightarrow \nu \psi \nu \leq \nu \iota \nu \\
& \Leftrightarrow \psi^{*} \geq \nu \nu \\
& \Leftrightarrow \psi^{*} \leq \iota \quad(\text { uma vez que } \nu \nu=\iota) .
\end{aligned}
$$

Um operator $\psi \in \Psi$ é dito ser uma abertura (respectivamente, um fechamento) se, e somente se, ele é crescente, anti-extensivo e idempotente (respectivamente, crescente, extensivo e idempotente). Note que, pela Proposição 3.18, $\psi$ é uma abertura se, e somente se, $\psi^{*}$ é um fechamento.

\subsection{Outros Reticulados Estudados}

Nas Subseções de 3.4.1 a 3.4.5, apresentamos cinco reticulados completos Booleanos estudados nesta tese. Veremos que os quatro reticulados apresentados nas Seções de 3.4.2 a 3.4.4 são isomorfos dois a dois. Dessa forma, um determinado problema definido em um 
desses reticulados pode ser estudado em qualquer um dos outros e, conseqüentemente, um resultado teórico obtido para um desses reticulados possui um equivalente em cada um dos outros.

\subsubsection{Reticulado de Conjuntos $\boldsymbol{P}(W)$}

Seja $W$ um subconjunto finito de $E$. O par $(\mathcal{P}(W), \subseteq)$ é um reticulado completo Booleano [14]. O maior e o menor elementos de $\mathcal{P}(W)$ são, respectivamente, $W$ e $\emptyset$. O ínfimo e o supremo de $X$ e $Y$ em $\mathcal{P}(W)$ são, respectivamente, $X \cap Y$ e $X \cup Y$. O conjunto complemento de $X \in \mathcal{P}(W)$ com respeito a $W$, denotado $X_{W}^{c}$ ou, simplesmente, $X^{c}$, quando o conjunto $W$ está claramente definido no contexto, é $X_{W}^{c}=\{x \in W: x \notin X\}$.

\subsubsection{Reticulado de Coleção de Conjuntos $P(P(W))$}

Seja $\mathcal{P}(\mathcal{P}(W))$ a coleção de todas subcoleções de $\mathcal{P}(W)$. Elementos de $\mathcal{P}(\mathcal{P}(W)$ ) serão denotados por letras caligráficas maiúsculas da forma $\mathcal{A}, \mathcal{B}, \mathcal{C}, \ldots$ O par $(\mathcal{P}(\mathcal{P}(W)), \subseteq)$ é um reticulado completo Booleano [12], onde a relação $\subseteq$ é definida por, para quaisquer $\mathcal{X}, \mathcal{Y} \in \mathcal{P}(\mathcal{P}(W)), \mathcal{X} \subseteq \mathcal{Y} \Leftrightarrow(X \in \mathcal{X} \Rightarrow Y \in \mathcal{Y})$. O maior e o menor elementos de $(\mathcal{P}(\mathcal{P}(W)), \subseteq)$ são, respectivamente, $\mathcal{P}(W)$ e $\emptyset$. O supremo e o ínfimo de $\mathcal{X}$ e $\mathcal{Y}$ em $\mathcal{P}(\mathcal{P}(W)$ ) são, respectivamente, $\mathcal{X} \cup \mathcal{Y}=\{X \in \mathcal{P}(W): X \in \mathcal{X}$ ou $X \in \mathcal{Y}\}$ e $\mathcal{X} \cap \mathcal{Y}=\{X \in \mathcal{P}(W): X \in \mathcal{X}$ e $X \in \mathcal{Y}\}$. O complemento de $\mathcal{X} \in \mathcal{P}(\mathcal{P}(W))$ com respeito a $\mathcal{P}(W)$ é $\mathcal{X}^{c}=\{X \in \mathcal{P}(W): X \notin \mathcal{X}\}$.

Para todo $\mathcal{X} \in \mathcal{P}(\mathcal{P}(W))$ e $h \in E, \mathcal{X}_{h} \in \mathcal{P}\left(\mathcal{P}\left(W_{h}\right)\right)$ denota a translação de todos elementos de $\mathcal{X}$ por $h$, ou seja, $\mathcal{X}_{h}=\left\{X_{h} \in \mathcal{P}\left(W_{h}\right): X \in \mathcal{X}\right\}$. Sejam $\mathcal{X}, \mathcal{Y} \in \mathcal{P}(\mathcal{P}(W))$. Claramente, se $\mathcal{X} \subseteq \mathcal{Y}$, então, para todo $h \in E, \mathcal{X}_{h} \subseteq \mathcal{Y}_{h}$. Usando este fato, temos a seguinte propriedade facilmente verificável.

Proposição 3.19 Sejam $\mathcal{X}, \mathcal{Y} \in \mathcal{P}(\mathcal{P}(W))$ e $C \in \mathcal{P}(E)$. Se $\mathcal{X} \subseteq \mathcal{Y}$, então

(a) $\cup\left\{\mathcal{X}_{h}: h \in C\right\} \subseteq \cup\left\{\mathcal{Y}_{h}: h \in C\right\}$,

(b) $\cap\left\{\mathcal{X}_{h}: h \in C\right\} \subseteq \cap\left\{\mathcal{Y}_{h}: h \in C\right\}$.

\subsubsection{Reticulado de Coleção de Intervalos Maximais}

Sejam $A, B \in \mathcal{P}(W)$ tais que $A \subseteq B$. Um intervalo contido em $\mathcal{P}(W)$ de extremidades $A$ e $B$ é o elemento $[A, B]$ de $\mathcal{P}(\mathcal{P}(W))$ dado por $[A, B]=\{X \in \mathcal{P}(W): A \subseteq X \subseteq B\}$. Os conjuntos $A$ e $B$ são chamados, respectivamente, as extremidades esquerda e direita do intervalo $[A, B]$. Para todos os pares $(A, B)$ tais que $A \nsubseteq B,[A, B]$ representa a coleção vazia.

Dado um elemento $\mathcal{X} \in \mathcal{P}(\mathcal{P}(W))$, podemos construir inúmeras coleções de intervalos. Por exemplo, $\{[X, X] \in \mathcal{P}(\mathcal{P}(W)): X \in \mathcal{X}\}$ ou $\{[A, B] \in \mathcal{P}(\mathcal{P}(W)):[A, B] \subseteq \mathcal{X}\}$. Note 

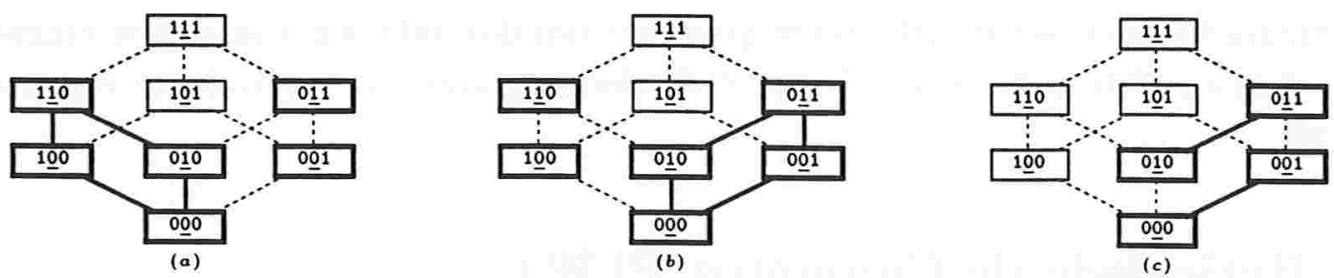

Figura 3.2: Diagramas que representam algumas coleções de um reticulado completo Booleano.

que uma coleção de intervalos é um subconjunto de $\mathcal{P}(\mathcal{P}(W)$ ), mas um subconjunto de $\mathcal{P}(\mathcal{P}(W))$ não é necessariamente uma coleção de intervalos. Nesta seção, vamos definir uma coleção de intervalos maximais e uma relação de ordem $\leq$ entre seus elementos. Veremos também que essa coleção munida com a relação $\leq$ é um reticulado completo Booleano.

Subconjuntos de $\mathcal{P}(\mathcal{P}(W))$ que são coleções de intervalos serão denotados por letras maiúsculas em negrito como $\mathbf{A}_{W}, \mathbf{B}_{W}, \mathbf{C}_{W}, \ldots$, ou, simplesmente, $\mathbf{A}, \mathbf{B}, \mathbf{C}, \ldots$, se o conjunto $W$ está claramente definido no contexto.

Dada uma coleção de intervalos $\mathbf{X}$ contidos em $\mathcal{P}(W)$, um intervalo $[A, B] \in \mathbf{X}$ é chamado intervalo maximal em $\mathbf{X}$ se, e somente se, não existe um outro intervalo $\left[A^{\prime}, B^{\prime}\right] \in$ $\mathbf{X}$, distinto de $[A, B]$, tal que $[A, B] \subseteq\left[A^{\prime}, B^{\prime}\right]$.

A coleção de todos intervalos maximais de $\mathbf{X}$ é denotada por $\operatorname{Max}(\mathrm{X})$. É claro que, se todos intervalos em $\mathbf{X}$ são maximais, então $\mathbf{X}=\operatorname{Max}(\mathbf{X})$.

Seja $\mathcal{X}$ um elemento de $\mathcal{P}(\mathcal{P}(W))$. A coleção de todos intervalos maximais que estão contidos em $\mathcal{X}$ é denotada $\mathbf{M}(\mathcal{X})$, ou seja, $\mathbf{M}(\mathcal{X})=\operatorname{Max}(\{[A, B] \subseteq \mathcal{P}(W):[A, B] \subseteq \mathcal{X}\})$.

Denotaremos por $\mathcal{U}(\mathrm{X})$ a coleção de todos elementos de $\mathcal{P}(W)$ que são elementos dos intervalos em $\mathbf{X}$, isto é, $\mathcal{U}(\mathbf{X})=\{X \in \mathcal{P}(W): X \in[A, B],[A, B] \in \mathbf{X}\}$.

Note que para qualquer $\mathcal{X} \subseteq \mathcal{P}(W), \mathcal{U}(\operatorname{Max}(\mathcal{X}))=\mathcal{X}$. Assim, qualquer coleção $\mathcal{X} \subseteq \mathcal{P}(W)$ pode ser representada por seus intervalos maximais.

Por simplicidade de notação, representaremos os subconjuntos de $W$ por sucessões de 's, 0's e 1's, onde '.' representa que o ponto não pertence a $W$, ' 0 ' denota que o ponto pertence a $W$ mas não pertence ao subconjunto e ' 1 ' significa que ele pertence ambos ao subconjunto e a $W$. Além disso, a origem nessa representação é mostrada por um caractere sublinhado. Por exemplo, se $W$ é o conjunto $\{(-1,0),(1,0)\}$, o subconjunto $\{(1,0)\}$ é representado por $0 \dot{\bullet} 1$

As subcoleções de um reticulado finito Booleano podem ser representadas por diagramas como os apresentados na Figura 3.2. As caixas representam os elementos do reticulado, e os segmentos de linhas tracejadas (isto é, arestas tracejadas) representam a ordem parcial. As caixas em negrito representam os elementos da subcoleção. Os intervalos maximais dentro de uma subcoleção são representados por arestas em negrito. 
Para uma maior clareza, se dois intervalos maximais de uma coleção têm uma aresta em comum, então eles serão representados em dois diagramas distintos.

Exemplo 3.20 Seja $W=1 \underline{11}$ e seja $\mathcal{X}=\{\underline{0} \underline{0}, 1 \underline{0} 0,0 \underline{10}, 0 \underline{0} 1,1 \underline{10}, 0 \underline{11}\}$. A coleção de todos intervalos maximais contidos em $\mathcal{X}$ é $\mathbf{M}(\mathcal{X})=\{[0 \underline{0} 0,1 \underline{10}],[0 \underline{0} 0,0 \underline{11}]\}$. As Figuras 3.2.a e 3.2.b mostram $\mathbf{M}(\mathcal{X})$.

Note que $\mathbf{M}(\mathcal{U}(\mathbf{X}))$ não é necessariamente igual a $\operatorname{Max}(\mathrm{X})$. Por exemplo, se $\mathbf{Y}=$ $\{[0 \underline{0} 0,0 \underline{0} 0],[0 \underline{0} 0,0 \underline{0} 1],[0 \underline{10} 0,0 \underline{11}]\}$, a coleção de intervalos maximais em Y é $\operatorname{Max}(\mathrm{Y})=$ $\{[0 \underline{0} 0,0 \underline{0} 1],[0 \underline{10} 0,0 \underline{1} 1]$,$\} e \mathbf{M}(\mathcal{U}(\mathbf{Y}))=\{[0 \underline{0} 0,0 \underline{11}]\}$. A Figura 3.2.c apresenta $\mathcal{U}(\mathrm{Y})$ e $\operatorname{Max}(\mathbf{Y})$.

Denotaremos por $\Pi_{W}$ o conjunto $\{\mathbf{M}(\mathcal{X}): \mathcal{X} \subseteq \mathcal{P}(W)\}$. Definimos a ordem parcial $\leq$ sobre os elementos de $\Pi_{W}$, estabelecendo que, para todo $\mathrm{X}, \mathrm{Y} \in \Pi_{W}$,

$$
\mathbf{X} \leq \mathbf{Y} \Leftrightarrow \forall[A, B] \in \mathbf{X}, \exists\left[A^{\prime}, B^{\prime}\right] \in \mathbf{Y}:[A, B] \subseteq\left[A^{\prime}, B^{\prime}\right]
$$

$\mathrm{O}$ poset $\left(\Pi_{W}, \leq\right)$ constitui um reticulado completo Booleano [12]. O supremo e o ínfimo de $\mathrm{X}$ e $\mathrm{Y}$ em $\Pi_{W}$ verificam, respectivamente, $\mathrm{X} \sqcup \mathrm{Y}=\mathrm{M}(\mathcal{U}(\mathrm{X}) \cup \mathcal{U}(\mathrm{Y}))$ e $\mathrm{X} \sqcap$ $\mathbf{Y}=\mathbf{M}(\mathcal{U}(\mathrm{X}) \cap \mathcal{U}(\mathbf{Y}))$. O complemento de $\mathrm{X} \in \Pi_{W}$ é denotado por $\overline{\mathrm{X}}$ e verifica $\overline{\mathrm{X}}=$ $\mathbf{M}\left((\mathcal{U}(\mathbf{X}))^{c}\right)$. Essas expressões valem, pois o mapeamento $\mathbf{M}(\cdot)$, definido de $\mathcal{P}(\mathcal{P}(W))$ em $\Pi_{W}$, é um isomorfismo de reticulado [12]. A inversa do mapeamento $\mathbf{M}(\cdot)$ é o mapeamento $\mathcal{U}(\cdot)[12]$. Com isto, podemos enunciar a seguinte proposição [12].

Proposição 3.21 O mapeamento $\mathbf{M}: \mathcal{P}(\mathcal{P}(W)) \rightarrow \Pi_{W}$, é um isomorfismo de reticulado entre $\mathcal{P}(\mathcal{P}(W))$ e $\Pi_{W}$. A inversa de $\mathbf{M}$ é o mapeamento $\mathcal{U}: \Pi_{W} \rightarrow \mathcal{P}(\mathcal{P}(W))$.

A Figura 3.4 apresenta um diagrama que mostra graficamente o isomorfismo de reticulado entre $(\mathcal{P}(\mathcal{P}(W)), \subseteq)$ e $\left(\Pi_{W}, \leq\right)$.

Para todo $\mathbf{X} \in \Pi_{W}$ e $h \in E, \mathbf{X}_{h} \in \Pi_{W_{y}}$ denota a translação de todos intervalos de $\mathbf{X}$ por $h$, isto é, $\mathbf{X}_{h}=\left\{\left[A_{h}, B_{h}\right] \in \Pi_{W_{h}}:[A, B] \in \mathbf{X}\right\}$.

Proposição 3.22 Se $\mathbf{X} \in \Pi_{W}$ e $h \in E$, então $(\mathbf{M}(\mathcal{U}(\mathbf{X})))_{h}=\mathbf{M}\left((\mathcal{U}(\mathrm{X}))_{h}\right)$.

Prova: Primeiramente, provaremos que $\mathcal{U}\left(\mathrm{X}_{h}\right)=(\mathcal{U}(\mathrm{X}))_{h}$. De fato,

$$
\begin{aligned}
D \in \mathcal{U}\left(\mathrm{X}_{h}\right) & \Leftrightarrow \exists[A, B] \in \mathbf{X}_{h}: D \in[A, B] \\
& \Leftrightarrow \exists[A, B] \in \mathbf{X}: D_{-h} \in[A, B] \\
& \Leftrightarrow D_{-h} \in \mathcal{U}(\mathbf{X}) \\
& \Leftrightarrow D \in(\mathcal{U}(\mathbf{X}))_{h} .
\end{aligned}
$$

Agora, uma vez que $\mathcal{U}\left(\mathrm{X}_{h}\right)=(\mathcal{U}(\mathrm{X}))_{h}$, então $\mathbf{M}\left(\mathcal{U}\left(\mathrm{X}_{h}\right)\right)=\mathbf{M}\left((\mathcal{U}(\mathrm{X}))_{h}\right)$ e, como $\mathbf{M}(\cdot)$ é a inversa de $\mathcal{U}(\cdot), \mathrm{X}_{h}=\mathbf{M}\left((\mathcal{U}(\mathrm{X}))_{h}\right)$.

Finalmente, uma vez que $\mathbf{X}=\mathbf{M}(\mathcal{U}(\mathrm{X}))$, então $(\mathbf{M}(\mathcal{U}(\mathrm{X})))_{h}=\mathbf{M}\left((\mathcal{U}(\mathrm{X}))_{h}\right)$. 


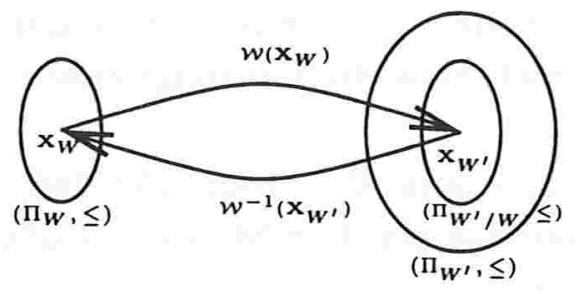

Figura 3.3: Isomorfismo de reticulado entre $\Pi_{W}$ and $\Pi_{W^{\prime} / W}$.

O próximo resultado é uma conseqüência imediata do isomorfismo de reticulado entre $(\mathcal{P}(\mathcal{P}(W)), \subseteq)$ e $\left(\Pi_{W}, \leq\right)$.

Proposição 3.23 Se $\mathrm{X}, \mathrm{Y}, \mathrm{Z} \in \Pi_{W}$ e $h \in E$, então
(a) $\mathrm{X} \sqcup \mathrm{Y}=\mathrm{Y} \sqcup \mathrm{X}$,
(b) $(\mathrm{X} \sqcup \mathrm{Y}) \sqcup \mathrm{Z}=\mathrm{X} \sqcup(\mathrm{Y} \sqcup \mathrm{Z})$,
(d) $\mathbf{X} \sqcap \mathrm{Y}=\mathrm{Y} \sqcap \mathbf{X}$,
(c) $(\mathrm{X} \sqcup \mathrm{Y})_{h}=\mathrm{X}_{h} \sqcup \mathrm{Y}_{h}$,
(e) $(\mathrm{X} \sqcap \mathrm{Y}) \sqcap \mathrm{Z}=\mathrm{X} \sqcap(\mathrm{Y} \sqcap \mathrm{Z})$,
(f) $(\mathbf{X} \sqcap \mathbf{Y})_{h}=\mathbf{X}_{h} \sqcap \mathbf{Y}_{h}$.

Prova: As propriedades $(a),(b),(d)$ e $(e)$ são imediatas, uma vez que $\left(\Pi_{W}, \leq\right)$ é um reticulado completo. Provaremos agora a propriedade $(c)$.

$$
\begin{aligned}
\left(\mathrm{X}_{h} \sqcup \mathrm{Y}_{h}\right) & =\mathrm{M}\left(\mathcal{U}\left(\mathrm{X}_{h}\right) \cup \mathcal{U}\left(\mathrm{Y}_{h}\right)\right) & & \text { (pela propriedade de isomorfismo) } \\
& =\mathbf{M}\left((\mathcal{U}(\mathbf{X}))_{h} \cup(\mathcal{U}(\mathbf{Y}))_{h}\right) & & \\
& =\mathbf{M}\left((\mathcal{U}(\mathbf{X}) \cup \mathcal{U}(\mathbf{Y}))_{h}\right) & & \\
& =(\mathbf{M}(\mathcal{U}(\mathbf{X}) \cup \mathcal{U}(\mathbf{Y})))_{h} & & \text { (pela Proposição 3.22) } \\
& =(\mathbf{X} \cup \mathbf{Y})_{h} & & \text { (pela propriedade de isomorfismo). }
\end{aligned}
$$

Analogamente à prova da propriedade $(c)$, pode-se provar a propriedade $(f)$.

Sejam $W, W^{\prime} \in \mathcal{P}(E)$ tais que $W \subseteq W^{\prime}$. Definimos o conjunto $\Pi_{W^{\prime} / W} \subseteq \Pi_{W^{\prime}}$ como $\Pi_{W^{\prime} / W}=\left\{\mathrm{X} \in \Pi_{W^{\prime}}: A^{\prime} \subseteq W\right.$ e $\left.B^{\prime}-W=W^{c}, \forall\left[A^{\prime}, B^{\prime}\right] \in \mathbf{X}\right\}$.

Proposição 3.24 Sejam $W^{\prime}, W \in \mathcal{P}(E)$ tais que $W \subseteq W^{\prime}$. O mapeamento $\mathcal{W}(\cdot)$, de $\left(\Pi_{W}, \leq\right)$ em $\left(\Pi_{W^{\prime} / W}, \leq\right)$, definido por

$$
\mathcal{W}\left(\mathrm{X}_{W}\right)=\left\{\left[A^{\prime}, B^{\prime}\right] \subseteq \mathcal{P}\left(W^{\prime}\right): A^{\prime}=A \text { e } B^{\prime}=B \cup W^{c},[A, B] \in \mathbf{X}_{W}\right\}
$$

constitui um isomorfismo de reticulado entre $\left(\Pi_{W}, \leq\right)$ e $\left(\Pi_{W^{\prime} / W}, \leq\right)$. A inversa do mapeamento $\mathcal{W}(\cdot)$ é o mapeamento $\mathcal{W}^{-1}(\cdot)$, de $\Pi_{W^{\prime} / W}$ em $\Pi_{W}$, definido por

$$
\mathcal{W}^{-1}\left(\mathrm{X}_{W^{\prime}}\right)=\left\{[A, B] \subseteq \mathcal{P}(W): A=A^{\prime} \text { and } B=B^{\prime} \cap W,\left[A^{\prime}, B^{\prime}\right] \in \mathbf{X}_{W^{\prime}}\right\}
$$

Prova: Se $\mathbf{X}_{W} \in \Pi_{W}$ e $[X, Y] \in \mathrm{X}_{W}$, então $X \subseteq Y \subseteq W$. Provaremos a bijeção entre $\Pi_{W}$ e $\Pi_{W^{\prime} / W}$. Primeiro, mostraremos que $\mathcal{W}(\cdot)$ é uma função injetora, isto é, para todo $\mathrm{X}_{W} \in \Pi_{W}$, temos que $\mathcal{W}^{-1}\left(\mathcal{W}\left(\mathrm{X}_{W}\right)\right)=\mathbf{X}_{W}$. Assim, 


$$
\begin{aligned}
& \mathcal{W}^{-1}\left(\mathcal{W}\left(\mathrm{X}_{W}\right)\right)=\left\{[A, B] \subseteq \mathcal{P}(W): A=A^{\prime}, B=B^{\prime} \cap W,\left[A^{\prime}, B^{\prime}\right] \in \mathcal{W}\left(\mathrm{X}_{W}\right)\right\} \\
& =\left\{[A, B] \subseteq \mathcal{P}(W): A=A^{\prime}, B=B^{\prime} \cap W,\right. \\
& \left.A^{\prime}=X, B^{\prime}=Y \cup W^{c},[X, Y] \in \mathrm{X}_{W}\right\} \\
& \text { (pela definição de } \mathcal{W}(\cdot) \text { ) } \\
& =\left\{[A, B] \subseteq \mathcal{P}(W): A=X, B=\left(Y \cup W^{c}\right) \cap W,[X, Y] \in \mathbf{X}_{W}\right\} \\
& =\left\{[A, B] \subseteq \mathcal{P}(W): A=X, B=Y,[X, Y] \in \mathrm{X}_{W}\right\} \\
& \text { (pela Proposição 2.4, uma vez que } Y \subseteq W \text { ) } \\
& =\left\{[A, B] \subseteq \mathcal{P}(W):[A, B] \in \mathbf{X}_{W}\right\} \\
& =\mathbf{X}_{W} \text {. }
\end{aligned}
$$

Agora, vamos provar que $\mathcal{W}(\cdot)$ é uma função sobrejetora, isto é, para todo $\mathrm{X}_{W^{\prime}} \in$ $\Pi_{W^{\prime} / W}$, temos que $\mathcal{W}\left(\mathcal{W}^{-1}\left(\mathrm{X}_{W^{\prime}}\right)\right)=\mathrm{X}_{W^{\prime}}$. Dessa forma,

$$
\begin{aligned}
& \mathcal{W}\left(\mathcal{W}^{-1}\left(\mathrm{X}_{W^{\prime}}\right)\right)=\left\{\left[A^{\prime}, B^{\prime}\right] \subseteq \mathcal{P}\left(W^{\prime}\right): A^{\prime}=A, B^{\prime}=B \cup W^{c},[A, B] \in \mathcal{W}^{-1}\left(\mathrm{X}_{W^{\prime}}\right)\right\} \\
& =\left\{\left[A^{\prime}, B^{\prime}\right] \subseteq \mathcal{P}\left(W^{\prime}\right): A^{\prime}=A, B^{\prime}=B \cup W^{c},\right. \\
& \left.A=X^{\prime}, B=Y^{\prime} \cap W,\left[X^{\prime}, Y^{\prime}\right] \in \mathrm{X}_{W^{\prime}}\right\} \\
& \text { (pela definição de } \mathcal{W}^{-1}(\cdot) \text { ) } \\
& =\left\{\left[A^{\prime}, B^{\prime}\right] \subseteq \mathcal{P}\left(W^{\prime}\right): A^{\prime}=X^{\prime}, B^{\prime}=\left(Y^{\prime} \cap W\right) \cup W^{c},\left[X^{\prime}, Y^{\prime}\right] \in \mathrm{X}_{W^{\prime}}\right\} \\
& =\left\{\left[A^{\prime}, B^{\prime}\right] \subseteq \mathcal{P}\left(W^{\prime}\right): A^{\prime}=X^{\prime}, B^{\prime}=Y^{\prime},\left[X^{\prime}, Y^{\prime}\right] \in \mathrm{X}_{W^{\prime}}\right\} \\
& \text { (pela Proposição 2.5, uma vez que } Y^{\prime} \subseteq W^{\prime} \text { e } Y^{\prime}-W=W^{c} \text { ) } \\
& =\left\{\left[A^{\prime}, B^{\prime}\right] \subseteq \mathcal{P}\left(W^{\prime}\right):\left[A^{\prime}, B^{\prime}\right] \in \mathbf{X}_{W^{\prime}}\right\} \\
& =\mathrm{X}_{W^{\prime}} \text {. }
\end{aligned}
$$

Logo, uma vez que $\mathcal{W}(\cdot)$ é injetora e sobrejetora, então, $\mathcal{W}(\cdot)$ é bijetora. Agora, vamos mostrar que $\mathcal{W}(\cdot)$ preserva a ordem parcial. Sejam $\mathbf{X}, \mathbf{Y} \in \Pi_{W}$.

$$
\begin{aligned}
\mathbf{X} \leq \mathbf{Y} & \Leftrightarrow \forall[A, B] \in \mathbf{X}, \exists[X, Y] \in \mathbf{Y}:[A, B] \subseteq[X, Y] \\
& \Leftrightarrow \forall[A, B] \in \mathbf{X}, \exists[X, Y] \in \mathbf{Y}:\left[A, B \cup W^{c}\right] \subseteq\left[X, Y \cup W^{c}\right] \\
& \Leftrightarrow \forall\left[A, B^{\prime}\right] \in \mathcal{W}(\mathbf{X}), \exists\left[X, Y^{\prime}\right] \in \mathcal{W}(\mathbf{Y}):\left[A, B^{\prime}\right] \subseteq\left[X, Y^{\prime}\right] \\
& \Leftrightarrow \mathcal{W}(\mathbf{X}) \leq \mathcal{W}(\mathbf{Y}) .
\end{aligned}
$$

Sejam $W, W^{\prime} \in \mathcal{P}(E)$ tais que $W \subseteq W^{\prime}$. A Figura 3.3 mostra um desenho que descreve graficamente o isomorfismo de reticulado entre $\left(\Pi_{W}, \leq\right)$ e $\left(\Pi_{W^{\prime} / W}, \leq\right)$.

Como conseqüência da Proposição 3.24, se $W \subseteq W^{\prime}$, então podemos mudar a representação de uma coleção de intervalos maximais $\mathrm{X}_{W} \in \Pi_{W}$ para $\mathrm{X}_{W^{\prime}} \in \Pi_{W^{\prime} / W} \subseteq \Pi_{W^{\prime}}$, e vice-versa.

Sejam $\mathrm{X}_{W} \in \Pi_{W}$ e $C \in \mathcal{P}(E)$. A adição e subtração de Minkowski de $\mathrm{X}_{W}$ por $C$ são, respectivamente, as coleções de intervalos maximais $\mathrm{X}_{W} \oplus C \in \Pi_{W \oplus C} \mathrm{e} \mathrm{X}_{W} \ominus C \in \Pi_{W \oplus C^{t}}$ dadas por $\mathbf{X}_{W} \oplus C=\sqcup\left\{\left(\mathbf{X}_{h}\right)_{W \oplus C}: h \in C\right\}$ e $\mathbf{X}_{W} \ominus C=\sqcap\left\{\left(\mathbf{X}_{-h}\right)_{W \oplus C^{t}}: h \in C\right\}$. 
Tecnicamente, a coleção $\mathrm{X}_{W} \oplus C \in \Pi_{W \oplus C}$ pode ser construída de seguinte forma. Para cada $h \in C$, faça a translação dos intervalos em $\mathbf{X}$ por $h$, para obter a coleção $\left(\mathrm{X}_{h}\right)_{W_{h}} \in \Pi_{W_{h}}$. Uma vez que $W_{h} \subseteq W \oplus C$, para todo $h \in C$, mude a representação de $\left(\mathrm{X}_{h}\right)_{W_{h}} \in \Pi_{W_{h}}$ para $\left(\mathrm{X}_{h}\right)_{W \oplus C} \in \Pi_{W \oplus C}$. Finalmente, obtenha o supremo das coleções $\left(\mathrm{X}_{h}\right)_{W \oplus C}$, para todo $h \in C$. A coleção $\mathrm{X} \ominus C$ é construída de forma similar. Por exemplo, sejam $W=1 \underline{11}$ e $\mathrm{X}_{W}=\{[\underline{0} \underline{0}, 0 \underline{0} 1],[0 \underline{0} 1,0 \underline{11}],[0 \underline{1} 1,1 \underline{11}]\}$. Se $C=1 \underline{10}$, então

$$
\begin{aligned}
\mathrm{X}_{W} \oplus C= & \{[00 \underline{0} 0,10 \underline{0} 1],[00 \underline{0} 1,10 \underline{1} 1],[00 \underline{1} 1,11 \underline{1} 1]\} \sqcup \\
& \{[00 \underline{0} 0,00 \underline{1} 1],[00 \underline{10} 0,01 \underline{1} 1],[01 \underline{10}, 11 \underline{11}]\} \\
= & \{[00 \underline{1} 0,01 \underline{1} 1],[00 \underline{0} 0,00 \underline{1} 1],[01 \underline{10}, 11 \underline{1} 1], \\
& {[00 \underline{0} 0,10 \underline{0} 1],[00 \underline{1} 1,11 \underline{1} 1],[00 \underline{0} 1,10 \underline{1} 1]\} }
\end{aligned}
$$

$\mathrm{e}$

$$
\begin{aligned}
\mathrm{X}_{W} \ominus C= & \{[0 \underline{0} 00,0 \underline{0} 11],[0 \underline{0} 10,0 \underline{1} 11],[0 \underline{11} 10,1 \underline{111}]\} \sqcap \\
& \{[0 \underline{0} 00,1 \underline{0} 01],[0 \underline{0} 01,1 \underline{0} 11],[\underline{0} 11,1 \underline{111}]\} \\
= & \{[0 \underline{1} 11,1 \underline{111}],[0 \underline{0} 11,0 \underline{1} 11],[0 \underline{0} 00,0 \underline{0} 01],[0 \underline{0} 01,0 \underline{0} 11]\}
\end{aligned}
$$

\subsubsection{Reticulado das Funções Binárias}

O conjunto de todas funções $f: \mathcal{P}(W) \rightarrow\{0,1\}$, denotado por $\{0,1\}^{\mathcal{P}(W)}$, é chamado de conjunto de funções binárias definidas sobre $\mathcal{P}(W)$. Definimos a ordem parcial $\leq$ sobre os elementos de $\{0,1\}^{\mathcal{P}(W)}$, estabelecendo que, para quaisquer $f, g \in\{0,1\}^{\mathcal{P}(W)}$, $f \leq g \Leftrightarrow f(X) \leq f(X)$, para todo $X \in \mathcal{P}(W)$. O par $\left(\{0,1\}^{\mathcal{P}(W)}, \leq\right)$ constitui um reticulado completo Booleano [12].

Um isomorfismo entre os reticulados $\left(\Pi_{W}, \leq\right)$ e $\left(\{0,1\}^{\mathcal{P}(W)}, \leq\right)$ é dada pela próxima proposição [12].

Proposição 3.25 Seja $F$ um mapeamento de $\Pi_{W}$ em $\{0,1\}^{\mathcal{P}(W)}$ definido por, para todo $X \in \mathcal{P}(W)$,

$$
F(\mathrm{X})(X)= \begin{cases}1 & \text { se } X \in \mathcal{U}(\mathrm{X}) \\ 0 & \text { caso contrário }\end{cases}
$$

constitui um isomorfismo de reticulado entre $\left(\Pi_{W}, \leq\right)$ e $\left(\{0,1\}^{\mathcal{P}(W)}, \leq\right)$. A sua inversa é definida por

$$
F^{-1}(f)=\mathbf{M}(\{X \in \mathcal{P}(W): f(X)=1\}) .
$$

A Figura 3.4 apresenta um diagrama que mostra graficamente o isomorfismo de reticulado entre $\left(\Pi_{W}, \leq\right)$ e $\left(\{0,1\}^{\mathcal{P}(W)}, \leq\right)$.

\subsubsection{Reticulado dos $W$-Operadores}

Um operador $\psi \in \Psi$ é chamado invariante por translação (IT) se, e somente se, para todo $x \in E$ e $X \in \mathcal{P}(E), \psi\left(X_{x}\right)=\psi(X)_{x}$. 
Seja $W$ um subconjunto finito de $E$. Um operador $\psi \in \Psi$ é chamado localmente definido (LD) na janela $W$ se, e somente se, para todo $x \in E$ e $X \in \mathcal{P}(E), x \in \psi(X) \Leftrightarrow$ $x \in \psi\left(X \cap W_{x}\right)$.

Um operador $\psi \in \Psi$ é chamado um $W$-operador se, e somente se, ele é IT e LD em uma janela $W$. O conjunto de todos $W$-operadores é denotado por $\Psi_{W}$. O par $\left(\Psi_{W}, \leq\right)$ constitui um sub-reticulado do reticulado $(\Psi, \leq)[12]$.

$\mathrm{Na}$ proposição [12] seguinte, apresentamos algumas propriedades de composições de $W$-operadores em janelas distintas.

Proposição 3.26 Sejam $\psi_{1}$ e $\psi_{2}$ dois $W$-operadores $L D$ 's em $W_{1}$ e $W_{2}$, respectivamente. Então,

(a) $\psi_{1} \vee \psi_{2}$ e $\psi_{1} \wedge \psi_{2}$ são $L D$ 's em $W_{1} \cup W_{2}$;

(b) $\psi_{2} \psi_{1}$ é $L D$ em $W_{1} \oplus W_{2}$;

(c) $\nu \psi_{1}, \psi_{1} \nu, \psi_{1} \iota, \iota \psi_{1}$ são $L D$ 's em $W_{1}$.

Note que, pela Proposição 3.26.c, os operadores $\nu$ e $\iota$ são neutros em relação ao tamanho da janela. Assim, obviamente, $\psi$ é um $W$-operador LD em $W$, se e somente se, $\psi^{*}$ é também um $W$-operador LD em $W$.

A seguir, estudamos alguns isomorfismos de reticulados envolvendo $\left(\Psi_{W}, \leq\right)$. Para isso, precisamos fazer a seguinte definição.

O núcleo ou kernel de um operator $\psi \in \Psi_{W}$ é o conjunto $\mathcal{K}_{W}(\psi) \in \mathcal{P}(W)$ dado por $\mathcal{K}_{W}(\psi)=\{X \in \mathcal{P}(W): o \in \psi(X)\}$.

Um isomorfismo entre os reticulados $(\mathcal{P}(\mathcal{P}(W)), \subseteq)$ e $\left(\Psi_{W}, \leq\right)$ é dado pela seguinte proposição [12].

Proposição 3.27 O mapeamento $\mathcal{K}_{W}(\cdot)$ de $\left(\Psi_{W}, \leq\right)$ em $(\mathcal{P}(\mathcal{P}(W)), \subseteq)$ constitui um isomorfismo de reticulado entre $\left(\Psi_{W}, \leq\right)$ e $(\mathcal{P}(\mathcal{P}(W)), \subseteq)$. A inversa do mapeamento $\mathcal{K}_{W}(\cdot)$ é o mapeamento $\mathcal{K}_{W}^{-1}(\cdot)$, onde $\mathcal{K}_{W}^{-1}(\cdot)$ é definido como $\mathcal{K}_{W}^{-1}(\mathcal{X})(X)=\left\{x \in E: X_{-x} \cap W \in\right.$ $\mathcal{X}\}$.

Um isomorfismo entre os reticulados $\left(\Psi_{W}, \leq\right)$ e $\left(\{0,1\}^{\mathcal{P}(W)}, \leq\right)$ é dado pela próxima proposição [12].

Proposição $3.28 O$ mapeamento $T$ de $\Psi_{W}$ em $\{0,1\}^{\mathcal{P}(W)}$ definido como, para todo, $X \in$ $\mathcal{P}(E)$,

$$
T(\psi)(X)= \begin{cases}1 & \text { se } o \in \psi(X) \\ 0 & \text { caso contrário }\end{cases}
$$

constitui um isomorfismo de reticulado entre $\left(\Psi_{W}, \leq\right)$ e $\left(\{0,1\}^{\mathcal{P}(W)}, \leq\right)$. A inversa do mapeamento $T$ é o mapeamento $T^{-1}$, definido como, para todo $X \in \mathcal{P}(E), T^{-1}(f)(X)=$ $\left\{x \in E: f\left(X_{-x} \cap W\right)=1\right\}$. 


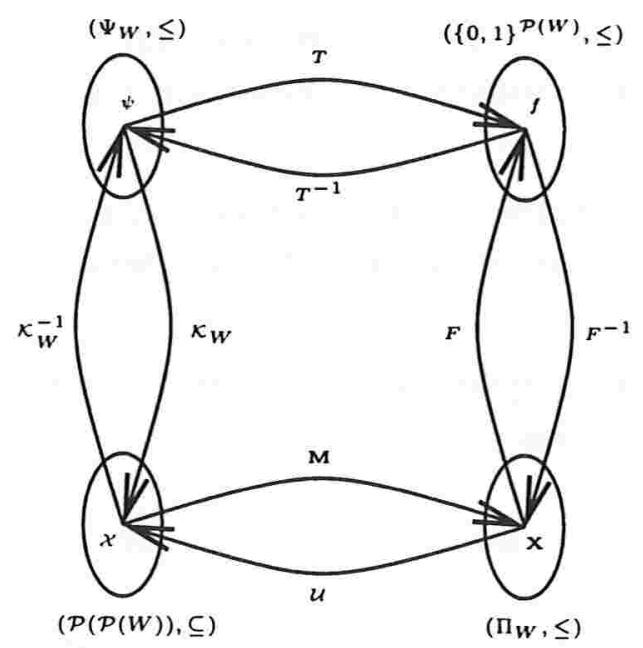

Figura 3.4: Os quatro isomorfismos de reticulados.

A Figura 3.4 apresenta um diagrama que mostra graficamente os isomorfismos entre os reticulados $(\mathcal{P}(\mathcal{P}(W)), \subseteq),\left(\Pi_{W}, \leq\right),\left(\{0,1\}^{\mathcal{P}(W)}, \leq\right)$ e $\left(\Psi_{W}, \leq\right)$.

Como estes reticulados são isomorfos dois a dois, temos que um determinado problema definido em um desses reticulados pode ser estudado em qualquer um dos outros. Assim, um resultado teórico em um desses reticulados possui um equivalente em cada um dos outros reticulados.

\subsection{Teorema da Decomposição de Operadores}

Um primeiro estudo sobre decomposição de operadores foi introduzido em um trabalho publicado por Matheron [32] onde foi estabelecido que qualquer operador IT e crescente é um supremo de operadores elementares denominados erosões, ou dualmente, um ínfimo de operadores elementares denominados dilatações. Uma generalização deste resultado para operadores IT's, mas não necessariamente crescentes, foi obtida por Banon e Barrera [6].

Nesta seção apresentaremos o estado da arte sobre decomposição de $W$-operadores. A maioria dos resultados apresentados nesta seção para $W$-operadores foram obtidos por Barrera e Salas [12].

\subsubsection{Operadores Elementares: Erosão e Dilatação}

Seja $C \in \mathcal{P}(E)$. A dilatação e erosão por $C$ são os operadores $\delta_{C}$ e $\varepsilon_{C}$ definidos por, para todo $X \in \mathcal{P}(E), \delta_{C}(X)=X \oplus C$ e $\varepsilon_{C}(X)=X \ominus C$. Se $C$ é um subconjunto finito, dizemos que ele é um elemento estruturante (EE). 
Devemos observar que $\delta_{C}$ e $\varepsilon_{C}$ são operadores IT's [24, p. 85][8, p. 71, Prop. 4.14]. Além disso, se $W$ é um subconjunto finito de $E$ e $C \in \mathcal{P}(W)$, então $\delta_{C}$ e $\varepsilon_{C}$ são $W$ operadores [12]. A seguir, apresentamos algumas propriedades de erosões e dilatações.

A próxima proposição [24, p. 85$]$ mostra que a dilatação e a erosão comutam, respectivamente, com a união e a intersecção.

Proposição 3.29 Se $A, B, C \in \mathcal{P}(E)$, então $\delta_{C}(A \cup B)=\delta_{C}(A) \cup \delta_{C}(B)$ e $\varepsilon_{C}(A \cap B)=$ $\varepsilon_{C}(A) \cap \varepsilon_{C}(B)$.

A proposição [24, p. 84, Eq. 4.41] seguinte mostra que o operador dual de uma erosão é uma dilatação e vice-versa.

Proposição $3.30 S e C \in \mathcal{P}(E)$, então $\delta_{C}^{*}=\varepsilon_{C^{t}}$ e $\varepsilon_{C}^{*}=\delta_{C^{t}}$.

$\mathrm{Na}$ proposição [12] seguinte, apresentamos uma propriedade de composição de um $W$-operador com uma dilatação ou com uma erosão.

Proposição 3.31 Se $\psi$ é um $W$-operador $L D$ em $W$, então os operadores $\delta_{C} \psi$ e $\varepsilon_{C} \psi$ são $L D$ 's, respectivamente, em $W \oplus C^{t}$ e $W \oplus C$.

Devemos observar que a Proposição 3.31 fornece o pior caso para o crescimento da janela na composição de $W$-operadores. Pode acontecer que uma composição, de dois operadores LD's em uma mesma janela $W$, produza um operador que ainda é LD em $W$. Por exemplo, se $\delta_{A} \varepsilon_{A}$ é LD em $W$, então $\left(\delta_{A} \varepsilon_{A}\right)\left(\delta_{A} \varepsilon_{A}\right)$ é também LD em $W$, uma vez que $\left(\delta_{A} \varepsilon_{A}\right)\left(\delta_{A} \varepsilon_{A}\right)=\delta_{A} \varepsilon_{A}$.

\subsubsection{Decomposição Canônica de $W$-Operadores}

Seja $W \subseteq E, W$ finito, e sejam $A, B \in \mathcal{P}(W)$ tais que $A \subseteq B$. O operador $\lambda_{A, B}^{W} \in \Psi_{W}$, definido por, para todo $X \in \mathcal{P}(E), \lambda_{A, B}^{W}(X)=\left\{x \in E: A \subseteq X_{-x} \cap W \subseteq B\right\}$, é denominado operador sup-gerador, ou simplesmente, sup-gerador. O operador $\mu_{A, B}^{W} \in \Psi_{W}$, definido por, para todo $X \in \mathcal{P}(E), \mu_{A, B}^{W}(X)=\left\{x \in E: X_{-x} \cap A^{t} \neq \emptyset\right.$ ou $\left(X_{-x} \cap W^{t}\right) \cup B^{t} \neq$ $\left.W^{t}\right\}$, é denominado operador inf-gerador, ou simplesmente, inf-gerador.

A seguinte proposição $[6,12]$ é uma propriedade dos operadores sup-geradores e infgeradores.

Proposição 3.32 Sejam $A, B \in \mathcal{P}(W)$ tais que $A \subseteq B$. Então, $\lambda_{A, B}^{W}=\varepsilon_{A} \wedge \nu \delta_{\left(B^{c}\right)^{t}} e$ $\mu_{A, B}^{W}=\delta_{A} \vee \nu \varepsilon_{\left(B^{c}\right)^{t}}$, onde o complemento de $B$ é em relação a $W$.

O próximo resultado $[6,12]$ mostra como é o núcleo de um sup-gerador $\lambda_{A, B}^{W}$. 
Proposição 3.33 O núcleo de um sup-gerador é um intervalo, isto é, $\mathcal{K}\left(\lambda_{A, B}^{W}\right)=[A, B]$.

A seguinte proposição [12] mostra que o operador dual de um sup-gerador é um infgerador e vice-versa.

Proposição 3.34 Se $C \in \mathcal{P}(E)$, então $\left(\lambda_{A, B}^{W}\right)^{*}=\mu_{A^{t}, B^{t}}^{W^{t}}$ e $\left(\mu_{A, B}^{W}\right)^{*}=\lambda_{A^{t}, B^{t}}^{W^{t}}$.

Banon e Barrera [6] mostraram uma conseqüência interessante da Proposição 3.32. Quando $B=W$ o operador sup-gerador $\lambda_{A, B}^{W}$ e o inf-gerador $\mu_{A, B}^{W}$ coincidem, respectivamente, com os operadores erosão e dilatação por $A$, isto é, $\lambda_{A, W}^{W}=\varepsilon_{A}$ e $\mu_{A, W}^{W}=\delta_{A}$.

Para não sobrecarregar a notação, denotaremos os operadores $\lambda_{A, B}^{W}$ e $\mu_{A, B}^{W}$ simplesmente por $\lambda_{A, B}$ e $\mu_{A, B}$, se o conjunto $W$ está claramente definido no contexto.

A decomposição de $W$-operadores em termos de sup-geradores (ou equivalentemente, em termos de operadores elementares) é apresentada pela sup-representação dada pelo seguinte teorema $[6,12]$.

Teorema 3.35 Se $\psi \in \Psi_{W}$, então $\psi=\bigvee\left\{\lambda_{A, B}:[A, B] \subseteq \mathcal{K}_{W}(\psi)\right\}$.

A decomposição de $W$-operadores em termos de inf-geradores é apresentada pela infrepresentação dada pelo seguinte teorema $[6,12]$.

Teorema 3.36 Se $\psi \in \Psi_{W}$, então $\psi=\wedge\left\{\mu_{A^{t}, B^{t}}^{W^{t}}:[A, B] \subseteq \mathcal{K}_{W}\left(\psi^{*}\right)\right\}$.

Pelo Teorema 3.35 (ou equivalentemente, pelo Teorema 3.36), o conjunto formado (i) pelos operadores erosão e dilatação, (ii) o operador de negação e (iii) as operações de intersecção e união é suficiente para representar qualquer $W$-operador. Este é o principal paradigma da MM e que pode ser formalizada pelo uso de uma linguagem formal, chamada aqui de linguagem morfológica (LM), cujo vocabulário compreende os operadores erosão e dilatação, o operador de negação, e as operações de intersecção e união. Esta linguagem é completa (isto é, ela é suficiente para descrever qualquer $W$-operador) e expressiva (isto é, a grande maioria dos $W$-operadores podem ser descritos por frases que usam relativamente poucas palavras). Uma frase da LM é denominada uma representação de um operador. Uma implementação da LM é chamada de máquina morfológica (MaqM) e um programa da MaqM é uma implementação de um operador para esta máquina.

\subsubsection{Decomposição Minimal de $W$-Operadores}

Um primeiro estudo sobre decomposição minimal de operadores IT's e crescentes, para decomposição apresentada por Matheron, foi introduzido por Maragos [30]. Seguindo 
a mesma idéia, Banon e Barrera [6] apresentaram uma generalização mostrando uma decomposição minimal para operadores IT's (não necessariamente crescentes).

A decomposição canônica de um $W$-operador (Teorema 3.35 ou 3.36) representa um resultado teórico bastante interessante. No entanto, com uma simples verificação, podemos observar que existem redundâncias nesta decomposição. Basta verificar que, se existem dois intervalos $[A, B]$ e $\left[A^{\prime}, B^{\prime}\right]$ contidos em $\mathcal{K}_{W}(\psi)$ tais que $[A, B] \subseteq\left[A^{\prime}, B^{\prime}\right]$, então o intervalo $[A, B]$ é redundante, pois

$$
\begin{aligned}
{[A, B] \subseteq\left[A^{\prime}, B^{\prime}\right] } & \Rightarrow \lambda_{A, B} \leq \lambda_{A^{\prime}, B^{\prime}} \\
& \Rightarrow \lambda_{A, B} \vee \lambda_{A^{\prime}, B^{\prime}}=\lambda_{A^{\prime}, B^{\prime}} .
\end{aligned}
$$

Assim, podemos definir um outro conjunto de intervalos equivalente ao núcleo, de cardinalidade menor ou igual, que também caracteriza a decomposição.

Para todo operador $\psi \in \Psi_{W}$, a base de $\psi$, denotada por $\mathbf{B}_{W}(\psi)$, ou simplesmente $\mathbf{B}(\psi)$ se $W$ está claramente definido no contexto, é a coleção de todos intervalos maximais contidos em $\mathcal{K}_{W}(\psi)$, isto é, $\mathbf{B}_{W}(\psi)=\mathbf{M}\left(\mathcal{K}_{W}(\psi)\right)$.

Observe que a cardinalidade da base de um $W$-operador $\psi$ é sempre menor ou igual à cardinalidade do seu núcleo.

A seguinte proposição mostra a base de um sup-gerador $\lambda_{A, B}^{W}$ e é uma conseqüência imediata da Proposição 3.33 .

Proposição 3.37 A base de um sup-gerador é composta por um único intervalo, isto é, $\mathbf{B}\left(\lambda_{A, B}^{W}\right)=\{[A, B]\}$.

O seguinte teorema [12] mostra que a decomposição de um $W$-operador $\psi$, dada pelo Teorema 3.35, pode ser simplificada usando a sua base em vez do seu núcleo.

Teorema 3.38 Se $\psi \in \Psi_{W}$, então $\psi=\bigvee\left\{\lambda_{A, B}:[A, B] \in \mathbf{B}_{W}(\psi)\right\}$.

A decomposição dada pelo Teorema 3.36 também pode ser simplificada [12].

Teorema 3.39 Se $\psi \in \Psi_{W}$, então $\psi=\bigwedge\left\{\mu_{A^{t}, B^{t}}^{W^{t}}:[A, B] \in \mathbf{B}_{W}\left(\psi^{*}\right)\right\}$.

A decomposição do operador $\psi \in \Psi_{W}$ dada nos Teoremas 3.38 e 3.39 são chamadas, respectivamente, de sup-representação ou sup-decomposição (minimal) e inf-representação ou inf-decomposição (minimal) de $\psi$.

\subsubsection{Propriedades da Base de um $W$-Operador}

Nesta seção, apresentaremos algumas propriedades da base de um $W$-operador. Com estas propriedades será possível encontrar a base de um $W$-operador $\psi$ a partir de qualquer representação de $\psi$. 
Dado um operador $\psi \in \Psi_{W}$ e um elemento $h \in E$, o operador $\psi_{h}$ é LD em $W_{h}$. O resultado seguinte [12] mostra como construir a base de $\psi_{h}$ a partir da base de $\psi$.

Proposição 3.40 Se $\psi \in \Psi_{W}$ e $h \in E$, então $\mathbf{B}_{W_{h}}\left(\psi_{h}\right)=\left(\mathbf{B}_{W}(\psi)\right)_{-h}$.

Dado um operador $\psi \in \Psi_{W}$ e $C \in \mathcal{P}(E)$, pela Proposição 3.31, os operadores $\delta_{C} \psi$ e $\varepsilon_{C} \psi$ são LD's, respectivamente, em $W \oplus C^{t}$ e $W \oplus C$. A proposição seguinte [12] mostra como construir as bases de $\delta_{C} \psi$ and $\varepsilon_{C} \psi$ a partir da base de $\psi$.

Proposição 3.41 Se $\psi \in \Psi_{W}$ e $C \in \mathcal{P}(E)$, então

$$
\begin{aligned}
& \mathbf{B}_{W \oplus C^{t}}\left(\delta_{C} \psi\right)=\mathbf{B}_{W}(\psi) \oplus C^{t}, \\
& \mathbf{B}_{W \oplus C}\left(\varepsilon_{C} \psi\right)=\mathbf{B}_{W}(\psi) \ominus C^{t} .
\end{aligned}
$$

Dados dois $W$-operadores $\psi_{1}, \psi_{2} \in \Psi_{W}$ tais que $\psi_{1}$ e $\psi_{2}$ são LD's, respectivamente, em $W_{1}$ e $W_{2}$, então, pela Proposição 3.26.a, os operadores $\psi_{1} \vee \psi_{2}$ e $\psi_{1} \wedge \psi_{2}$ são LD's em $W_{1} \cup W_{2}$. A proposição seguinte [12] mostra como construir as bases de $\psi_{1} \vee \psi_{2}$ e $\psi_{1} \wedge \psi_{2}$ a partir das bases de $\psi_{1}$ e $\psi_{2}$.

Proposição 3.42 Se $\psi_{1}$ e $\psi_{2}$ são dois $W$-operadores LD's, respectivamente, em $W_{1}$ e $W_{2}$, então

$$
\begin{aligned}
& \mathbf{B}_{W_{1} \cup W_{2}}\left(\psi_{1} \vee \psi_{2}\right)=\mathbf{B}_{W_{1}}\left(\psi_{1}\right) \sqcup \mathbf{B}_{W_{2}}\left(\psi_{2}\right), \\
& \mathbf{B}_{W_{1} \cup W_{2}}\left(\psi_{1} \wedge \psi_{2}\right)=\mathbf{B}_{W_{1}}\left(\psi_{1}\right) \sqcap \mathbf{B}_{W_{2}}\left(\psi_{2}\right) .
\end{aligned}
$$

Na proposição seguinte [12], mostramos como construir a base da composição de um $W$-operador $\psi$ com o operador de negação, a partir da base de $\psi$.

Proposição 3.43 Se $\psi \in \Psi_{W}$, então $\mathbf{B}_{W}(\nu \psi)=\overline{\mathbf{B}_{W}(\psi)}$.

Com os resultados apresentados nas Proposições 3.41, 3.42 e 3.43 podemos construir a base de qualquer $W$-operador $\psi$ a partir de uma representação de $\psi$, ou seja, de uma frasa da LM que representa $\psi$ [12]. A idéia é utilizar estes resultados recursivamente para construir passo a passo (incrementalmente) a base de $\psi$. Neste processo, a condição inicial é a base do operador identidade, $\mathbf{B}_{W}(\iota)=\{[\{o\}, W]\}$. Um estudo mais aprofundado deste resultado pode ser encontrado em [12].

Como conseqüência da construção incremental da base de um operador $\psi$ a partir de qualquer representação $\psi$, podemos verificar se duas frases da LM são equivalentes ou sinônimas, uma vez que estrutura de decomposição, dada pelo Teorema 3.38, caracteriza unicamente um $W$-operador, isto é, se duas representações de operadores têm a mesma base, então estas elas representam, de fato, o mesmo operador.

Para finalizar esta seção, apresentamos mais uma propriedade da base de $W$-operadores [12]. Seja $\psi \in \Psi_{W}$. Através desta propriedade podemos construir a base de $\psi^{*}$ a partir da base de $\psi$. 
Proposição 3.44 Seja $I=\{1,2, \cdots, n\}$ um conjunto de indices. Seja $\psi \in \Psi_{W}$. Se $\mathrm{B}(\psi)=\left\{\left[A_{i}, B_{i}\right]: i \in I\right\}$, então a base de $\psi^{*} e ́$

$$
\mathbf{B}\left(\psi^{*}\right)=\overline{\sqcup\left\{\left\{\left[B_{i}^{c}, A_{i}^{c}\right]\right\}: i \in I\right\}} .
$$

\subsection{Decomposição de Operadores Especiais}

Para algumas classes de operadores, devido a certas propriedades algébricas, podemos obter estruturas de decomposição mais simples do que a decomposição canônica. Nesta seção, apresentamos as decomposições de duas classes particulares de operadores obtidas por Matheron. Na Subseção 3.6.1 apresentamos a decomposição de operadores crescentes e IT's. Na Subseção 3.6.2, apresentamos a decomposição das aberturas e fechamentos IT's.

\subsubsection{Operadores Crescentes Invariantes por Translação}

Seja $\psi \in \Psi_{W}$ um operador crescente. A definição de operadores crescentes implica que seu núcleo tem a propriedade de herança, ou seja, para todo $X, Y \in \mathcal{P}(W)$ tais que $X \subseteq Y$, se $X \in \mathcal{K}(\psi)$, então $Y \in \mathcal{K}(\psi)$. Logo, para todo $[A, B] \in \mathbf{B}(\psi)$, temos que $B=W$ e os operadores $\lambda_{A, B}$ e $\mu_{A, B}$ das famílias de sup-geradores e inf-geradores dos Teoremas 3.35 e 3.36 tornam-se, respectivamente, erosões e dilatações [6]. Dessa forma, temos o seguinte resultado.

Teorema 3.45 Seja $\psi \in \Psi_{W}$. Se $\psi$ é crescente, então

$$
\begin{aligned}
& \text { (a) } \psi=\bigvee\left\{\varepsilon_{A}:[A, W] \in \mathbf{B}_{W}(\psi)\right\} \\
& \text { (b) } \psi=\bigwedge\left\{\delta_{A^{t}}:[A, W] \in \mathbf{B}_{W}\left(\psi^{*}\right)\right\} .
\end{aligned}
$$

Se $W=E$, a classe dos $W$-operadores é ela mesma a classe de operadores IT's, e sob esta condição, o resultado de decomposição apresentado no Teorema 3.45 é o resultado clássico da decomposição de operadores crescentes e IT's obtida por Matheron [32].

Como a base de um operador $\psi \in \Psi_{W}$ se simplifica no caso em que $\psi$ é crescente, ou seja, a extremidade direita de todo intervalo na base de $\psi$ é a própria janela $W$, então a Proposição 3.44 pode ser facilmente simplificada para o seguinte resultado.

Proposição 3.46 Seja $I=\{1,2, \cdots, n\}$ um conjunto de índices. Seja $\psi \in \Psi_{W}$ um operador crescente. Se $\mathbf{B}(\psi)=\left\{\left[A_{i}, W\right]: i \in I\right\}$, então a base de $\psi^{*}$ é

$$
\mathbf{B}\left(\psi^{*}\right)=\sqcap\left\{[\{a\}, W]: a \in A_{i}^{c} \text { e } i \in I\right\} .
$$




\subsubsection{Aberturas e Fechamentos Invariantes por Translação}

Para cada $A \in \mathcal{P}(W)$, a abertura morfológica $\gamma_{A}$ (respectivamente, fechamento morfológico $\phi_{A}$ ) é o operador construído pela composição da erosão $\varepsilon_{A}$ (respectivamente, da dilatação $\delta_{A}$ ) com a dilatação $\delta_{A}$ (respectivamente, da erosão $\varepsilon_{A}$ ), ou seja, $\gamma_{A}=\delta_{A} \varepsilon_{A}$ (respectivamente, $\phi_{A}=\varepsilon_{A} \delta_{A}$ ).

O núcleo de uma abertura morfológica é dado pela seguinte proposição.

Proposição 3.47 Se $A \in \mathcal{P}(W)$, então $\mathcal{K}_{W}\left(\gamma_{A}\right)=\left\{X \in \mathcal{P}(W): A_{-h} \subseteq X\right.$ e $\left.h \in A\right\}$.

Prova: Pela definição de $\gamma_{A}$ e pela Proposição 3.12, temos que, para todo $X \in \mathcal{P}(E)$, $\gamma_{A}(X)=\cup\left\{A_{h}: h \in E\right.$ e $\left.A_{h} \subseteq X\right\}$. Assim, para todo $Y \in \mathcal{P}(W)$,

$$
\begin{aligned}
Y \in \mathcal{K}\left(\gamma_{A}\right) & \Leftrightarrow o \in \gamma_{A}(Y) \\
& \Leftrightarrow o \in \cup\left\{A_{h}: h \in E \text { e } A_{h} \subseteq Y\right\} \\
& \Leftrightarrow \exists h \in E: o \in A_{h} \text { e } A_{h} \subseteq Y \\
& \Leftrightarrow \exists h \in E: h \in A \text { e } A_{-h} \subseteq Y \\
& \Leftrightarrow \exists h \in A: A_{-h} \subseteq Y \\
& \Leftrightarrow Y \in\left\{X \in \mathcal{P}(W): A_{-h} \subseteq X \text { e } h \in A\right\} .
\end{aligned}
$$

Uma abertura morfológica (respectivamente, um fechamento morfológico) é independente da translação, isto é, para todo $h \in E, \gamma_{A}=\gamma_{A_{h}}$ (respectivamente, $\phi_{A}=\phi_{A_{h}}$ ).

Um invariante de um operador $\psi \in \Psi$ é um subconjunto $X \in \mathcal{P}(E)$ tal que $\psi(X)=X$. O conjunto de todos invariantes de $\psi$ será denotado por $\operatorname{Inv}(\psi)$, isto é, $\operatorname{Inv}(\psi)=\{X \in$ $\mathcal{P}(E): \psi(X)=X\}$.

Matheron [32, Prop. 7.1.3] provou a seguinte decomposição para aberturas e fechamentos IT's.

Teorema 3.48 Seja $\psi \in \Psi$.

(a) Se $\psi$ é uma abertura IT, então $\psi=\bigvee\left\{\gamma_{A}: A \in \operatorname{Inv}(\psi)\right\}$

(b) Se $\psi$ é um fechamento IT, então $\psi=\bigwedge\left\{\phi_{A^{t}}: A \in \operatorname{Inv}\left(\psi^{*}\right)\right\}$.

Devemos alertar o leitor para não confundir a definição de invariante de um conjunto (definido na Subseção 3.2.1) com invariante de um operador (definido acima). Lembramos também que na Subseção 3.2.1, definimos que um conjunto $A \in \mathcal{P}(W)$ é um aberto de $B \in \mathcal{P}(W)$ se, e somente se, $A$ é um invariante de $B$. Os invariantes (ou equivalentemente, os abertos) de um determinado conjunto têm a seguinte propriedade [41, p. 54].

Proposição 3.49 Sejam $A, B \in \mathcal{P}(W)$. Se $A$ é um invariante de $B$, então $\gamma_{B} \leq \gamma_{A} e$ $\phi_{A} \leq \phi_{B}$.

A propriedade dada pela Proposição 3.49 permite a construção de granulometrias [43] e, por esta razão, esta propriedade é chamada de granulometria de absorção. 


\subsection{Mudança de Estrutura de Representação}

Para um $W$-operador $\psi$ podem existir inúmeras frases sinônimas da LM que o representem. Na Subseção 3.5.4, vimos algumas propriedades para construir incrementalmente a base de um operador $\psi$ a partir de uma representação de $\psi$. Nesta seção apresentaremos o estado da arte no que se refere ao problema de transformação de representação de um operador $\psi$, a partir de sua base, para encontrar frases da LM que implementem eficientemente o operador $\psi$. Este problema é extremamente complexo e pode ser visto como o problema inverso da construção incremental da base.

A escolha de "boas" representações de um operador depende da arquitetura da máquina na qual o operador será implementado. Em geral, decomposições seqüênciais são mais adequadas para máquinas convencionais, enquanto que decomposições híbridas (seqüencial-paralela) são mais adequadas para máquinas paralelas.

Atualmente, na literatura, existem poucos artigos que estudaram o problema geral de transformação de representação de um operador, uma vez que se trata de um problema extremamente difícil. Um primeiro trabalho, feito por Jones [27], foi o estudo de mudança de representação de operadores IT's e crescentes. O problema estudado no artigo do Jones pode ser enunciado da seguinte forma: dada a sup-decomposição de um operador IT e crescente $\psi_{1}$, encontrar todos subconjuntos possíveis para $C \in \mathcal{P}(E)$ e de todas possíveis sup-decomposições dos operadores $\psi_{2}$ e $\psi_{3}$, também IT's e crescentes, tais que verifiquem uma das seguintes equações:
(a) $\psi_{1}=\delta_{C} \psi_{2} \vee \psi_{3}$
(b) $\psi_{1}=\delta_{C} \psi_{2} \wedge \psi_{3}$
(c) $\psi_{1}=\varepsilon_{C} \psi_{2} \vee \psi_{3}$
(d) $\psi_{1}=\varepsilon_{C} \psi_{2} \wedge \psi_{3}$.

No trabalho do Jones são apenas apresentados, para cada equação, alguns limites superiores e inferiores para o subconjunto $C$ e para as sup-decomposições de $\psi_{2}$ e $\psi_{3}$, com o objetivo de diminuir o espaço de busca das soluções das equações apresentadas acima. No entanto, ele não fez um estudo detalhado do problema, apresentando os resultados de uma maneira bastante intrincada.

\subsubsection{Mudança de Representação de Erosões e Dilatações}

Uma vez que, no caso geral, o problema de encontrar a melhor representação de um operador é extremamente difícil, restringimos o estudo das propriedades da sup-decomposição para certas classes de operadores. De fato, existe um número considerável de estudos desse problema para a família das erosões e dilatações.

Uma propriedade de dilatações e erosões é que elas podem ter uma decomposição seqüencial [41, p. 47]. 


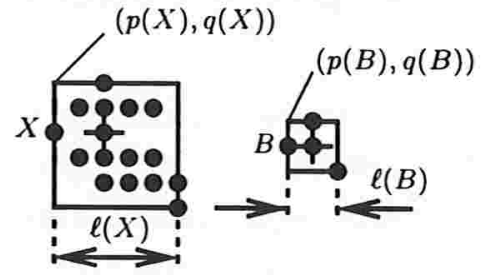

(a)

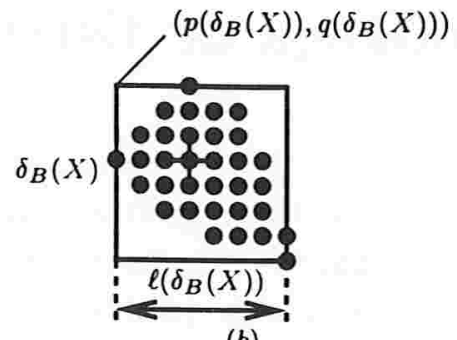

(b)

Figura 3.5: (a) Os conjuntos $X$ e $B$. (b) Dilatação de $X$ por $B$.

Proposição 3.50 Sejam $A, B_{1}, B_{2}, \cdots, B_{n} \in \mathcal{P}(E)$. Então, $\delta_{A}=\delta_{B_{1}} \delta_{B_{2}} \cdots \delta_{B_{n}}$ e $\varepsilon_{A}=$ $\varepsilon_{B_{1}} \varepsilon_{B_{2}} \cdots \varepsilon_{B_{n}}$ se, e somente se, $A=B_{1} \oplus B_{2} \oplus \cdots \oplus B_{n}$.

Teoricamente, a decomposição seqüencial de erosões e dilatações pode ser considerada a partir de dois pontos de vista. Por um lado, considerando a sup-decomposição (respectivamente, inf-decomposição), uma erosão (respectivamente, dilatação) é o operador mais simples que pode ser representado, uma vez que a sup-decomposição (respectivamente, inf-decomposição) de uma erosão (respectivamente, dilatação) é uma erosão (respectivamente, dilatação) [24, p. 86, Teorema 4.15] (veja também o Teorema 3.45). Por outro lado, a composição de erosões (respectivamente, dilatações) é equivalente à erosão (respectivamente, dilatação) de adições acumuladas de Minkowski de EE's que caracterizam as erosões (respectivamente, dilatações) [41, p. 47] (veja também a Proposição 3.50). Portanto, o problema de transformar a sup-decomposição de uma erosão (respectivamente, inf-decomposição de uma dilatação) em uma decomposição seqüencial é equivalente a encontrar uma decomposição do EE que o caracteriza em termos de adições de Minkowski.

Para o estudo de decomposição de erosões e dilatações, consideraremos $E=\mathbb{Z}^{2}$ e o ponto $o=(0,0)$ como a origem de $\mathbb{Z}^{2}$. O quadrado $3 \times 3$ centrado na origem de $\mathbb{Z}^{2}$, isto é, o conjunto $\{-1,0,1\}^{2}=\{-1,0,1\} \times\{-1,0,1\}$, é chamado de quadrado elementar.

Seja $X \subseteq \mathbb{Z}^{2}$ e seja $n$ um inteiro em $\mathbb{Z}_{+}$. A sucessão de $n-1$ adições de Minkowski $((X \oplus X) \oplus \cdots \oplus X)$ é denotada por $n X$. Essa notação é estendida para $n=0$ estabelecendo que $0 X=\{o\}$.

Sejam $X, B \subseteq \mathbb{Z}^{2}$. Para se computar uma dilatação $\left(\delta_{B}(X)\right)$ ou uma erosão $\left(\varepsilon_{B}(X)\right)$, adotaremos a seguinte estrutura de dados para armazenar $X$ e $B$.

Seja $\ell(X)$ (respectivamente, $\ell(B)$ ) o comprimento do menor quadrado que contém $X$ (respectivamente, o comprimento do menor quadrado que contém $B)$ e $(p(X), q(X))$ (respectivamente, $(p(B), q(B))$ ) o ponto correspondente à extremidade superior esquerda deste quadrado. Por exemplo, para o conjunto $X$ na Figura 3.5. $a, \ell(X)=5, p(X)=-2$ e $q(X)=2$.

O EE $B$ será representado por uma estrutura de dados composta pelo valor $\ell(B)$, pela coordenada do ponto $(p(B), q(B))$ e uma lista ligada que contém os valores das 
coordenadas de cada ponto pertencente a $B$. Por exemplo, a estrutura de dados para armazenar o EE $B$ da Figura 3.5. $a$ contém os valores $\ell(B)=2, p(B)=-1, q(B)=1 \mathrm{e}$ uma lista ligada contendo os pontos $(0,1),(-1,0),(0,0)$ e $(1,-1)$.

A estrutura de dados para armazenar a imagem de entrada é composta por uma matriz que contém valores 0 's e 1's $(X[i, j]=1$ se, e somente se, o ponto $(i, j)$ pertence a $X)$, pelo comprimento $\ell(X)$ e pela coordenada do ponto $(p(X), q(X))$.

Pode-se mostrar que $\ell\left(\delta_{B}(X)\right)=\ell(X)+\ell(B), p\left(\delta_{B}(X)\right)=p(X)+p(B)$ e $q\left(\delta_{B}(X)\right)=$ $q(X)+q(B)$ (veja Proposição 4.17). Por exemplo, na Figura 3.j.b,

$$
\begin{aligned}
& \ell\left(\delta_{B}(X)\right)=\ell(X)+\ell(B)=5+2=7 \\
& \text { - } p\left(\delta_{B}(X)\right)=p(X)+p(B)=-2+(-1)=-3 ; \\
& \text { - } q\left(\delta_{B}(X)\right)=q(X)+q(B)=2+1=3 .
\end{aligned}
$$

Apresentamos a seguir um algoritmo simples para computar uma dilatação.

Entrada: Um conjunto $X \subseteq \mathbb{Z}^{2}$ e um EE $B \subseteq \mathbb{Z}^{2}$.

Saída: $\mathrm{O}$ conjunto $C \subseteq \mathbb{Z}^{2}$ correspondente à dilatação $\delta_{B}(X)$.

01: $\quad / *$ Inicialização do conjunto $C * /$

02: $\quad p(C)=p(X)+p(B) ; q(C)=q(X)+q(B) ;$

03: $\quad \ell(C)=\ell(X)+\ell(B)$;

04: $\quad$ ara cada $i$ de $p(C)$ a $p(C)+\ell(C)$ faça

05: $\quad$ para cada $j$ de $p(C)$ a $q(C)+\ell(C)$ faça

06: $\quad C[i, j] \leftarrow 0$;

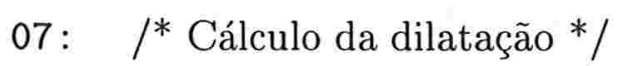

08: $\quad$ para cada $(u, v)$ da lista de pontos de $B$ faça

09: $\quad$ para cada $i$ de $p(X)$ a $p(X)+\ell(X)$ faça

10: $\quad$ para cada $j$ de $p(X)$ a $q(X)+\ell(X)$ faça

11: $\quad C[i, j] \leftarrow C[i, j]$ OR $X[i+u, j+v]$

A operação OR no Passo 11 denota a operação de "ou" lógico.

Assim, a complexidade de tempo para se computar $\delta_{B}(X)$ é igual a $\Theta\left((\ell(B)+\ell(X))^{2}+\right.$ $\left.|B| \cdot \ell(X)^{2}\right)$.

A decomposição seqüencial de EE's tem implicação na complexidade das implementações da erosão e dilatação. Normalmente, a implementação de seqüências de erosões e dilatações é mais rápida e simples do que a equivalente implementação direta.

Por exemplo, se $B$ é o quadrado elementar, facilmente podemos verificar que, para 


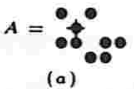

(a)

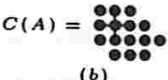

(b)

Figura 3.6: (a) Um EE $A$. (b) O fecho convexo de $A$.

$n>0,|n B|=(2 n+1)^{2}$ e $\ell(n B)=2 n+1$. Uma vez que $|B|=9$ e $\ell(B)=2$, então a complexidade para computar $\delta_{B}(X)$ é $\Theta\left(m^{2}\right)$, onde $m=\ell(X)$. Assim, as complexidades para computar $\delta_{n B}$ e $\delta_{B} \delta_{B} \cdots \delta_{B}$ são, respectivamente, $\Theta\left(n^{2} \cdot m^{2}\right)$ e $\Theta\left(n \cdot m^{2}+n^{2} \cdot m+\right.$ $\left.n^{3}\right)$. Observe que se $n=O\left(m^{2}\right)$, então a implementação seqüencial é melhor que a implementação direta. Além disso, a implementação seqüencial é mais eficiente para máquinas com "hardware" especializado onde em geral há uma operação em "hardware" para executar rapidamente dilatações e erosões por EE's que são subconjuntos do quadrado elementar.

Na prática, podemos considerar que o conjunto $X \subseteq \mathbb{Z}^{2}$ tem sempre uma dimensão constante (por exemplo, $256 \times 256$ pixels) e, portanto, qualquer operação de translação, união e intersecção em $X$ é feito em tempo constante. Dessa forma, podemos calcular o número de operações efetuadas para se computar uma dilatação $\delta_{B}(X)$ levando em conta somente o número de operações uniões (ou equivalentemente, o número de pontos de $B$ ). Assim, neste caso, a medida de complexidade para se computar uma dilatação $\delta_{B}(X)$ (respectivamente, uma erosão $\varepsilon_{B}(X)$ ) depende somente da cardinalidade de $B$.

Voltemos ao exemplo em que $B$ é o quadrado elementar. Uma vez que $|B|=9$, então a computação de $\delta_{B}(X)$ é feito em tempo constante. Como, para $n>0,|n B|=(2 n+1)^{2}$. então, as complexidades para computar $\delta_{n B}(X)$ e $\delta_{B} \delta_{B} \cdots \delta_{B}(X)$ são, respectivamente, $(2 n+1)^{2}$ e $9 \cdot n$. De fato, a complexidade de computar erosões e dilatações por um EE decomposto como seqüência de adições de Minkowski em termos de subconjuntos do quadrado elementar, em uma máquina convencional, foi quantitativamente estudado por Maragos [30, p. 77], que mostrou exemplos onde a complexidade de tempo de algoritmos que implementam erosões e dilatações foram, de quadráticas, na implementação direta, para linear, na decomposição do EE por adições de Minkowski.

Alguns outros exemplos de como a complexidade de tempo de implementações de erosões e dilatações diminui, quando se usa decomposição seqüencial em vez de usar a implementação direta, podem ser encontrados em [8, p. 162].

Muitos pesquisadores [51, 38, 49, 28, 34, 35, 50, 1, 20, 19, 47, 45, 44, 33] têm estudado o problema de decomposição de um EE $A$ como seqüência de adições de Minkowski de subconjuntos de cardinalidade menores que $A$ e propuseram diferentes algoritmos para gerarem tais decomposições.

Zhuang and Haralick [51] apresentaram um algoritmo baseado em uma árvore de busca para decomposição de um EE arbitrário, onde todos elementos na decomposição têm um número $k$ pré-estabelecido e fixo de pontos.

Devemos observar que nem todos EE's têm decomposição seqüêncial por adições de 
Minkowski [49]. Além disso, o problema geral de decomposição de EE's é NP-completo [33]. No entanto, sabe-se que todo EE convexo têm uma decomposição seqüêncial [49]. Um EE convexo é definido a seguir.

O fecho convexo $C(A)$ de um EE $A$ é a intersecção de todos semi-planos que contém $A$. Nesta tese, vamos supor que os subconjuntos estão representados em uma grade quadrada e consideraremos que somente os semi-planos com inclinações 0, 45, 90 e 135 graus para construir o fecho convexo (para um exemplo, veja a Figura 3.6). Um EE $A$ é dito convexo se, e somente se, $A=C(A)$.

Em 1991, Xu [49] apresentou um algoritmo para decomposição de EE's convexos em termos de um número mínimo de subconjuntos convexos do quadrado elementar.

Park e Chin [35] apresentaram uma extensão do algoritmo de Xu para decompor EE's simplesmente conexos (isto é, um EE 8-conexo que não contém buracos ${ }^{1}$ ), onde todos elementos na decomposição são também simplesmente conexos. Observe que todo EE convexo é também simplesmente conexo.

\subsection{Problemas Estudados nesta Tese}

Após uma explanação de alguns aspectos do estado da arte em MM, apresentaremos, nesta seção, os problemas que foram estudados nesta tese. A partir do próximo capítulo, todos resultados apresentados são contribuições originais desta tese. Acreditamos que com esses resultados foram acrescentados alguns avanços no estado da arte em MM no que se refere à transformação de estruturas de decomposição.

\subsubsection{Decomposição de Elementos Estruturantes}

Nesta tese, estudamos o problema de decompor EE's convexos, simplesmente conexos e arbitrários. Os detalhes são dados nas subseções a seguir.

\subsubsection{Decomposição de Elementos Estruturantes Convexos}

Para o caso de EE's convexos, avançamos no estado da arte em MM apresentando no Capítulo 5 um novo algoritmo que usa uma estratégia gulosa para decompor tais EE's.

\subsubsection{Decomposição de Elementos Estruturantes Simplesmente Conexos}

Para o caso de EE's simplesmente conexos, mostramos, no Capítulo 6, que o algoritmo de Park e Chin [35] para decompor tais EE's não decompõe três famílias infinitas de EE's

\footnotetext{
${ }^{1}$ Um estudo aprofundado sobre conexidade e topologia digital pode ser encontrado em [29].
} 
decomponíveis simplesmente conexos.

\subsubsection{Decomposição de Elementos Estruturantes Arbitrários}

No caso geral, apresentamos no Capítulo 7 um algoritmo que usa uma estratégia de "branch and bound" para decompor EE's arbitrários.

\subsubsection{Representação Compacta de $W$-Operadores}

Nesta tese, no Capítulo 8, estudamos o problema de representação de operadores e introduzimos uma nova representação mais compacta (ou seja, que utiliza um número menor de operadores elementares) que a decomposição na forma canônica. Este estudo é apresentado de uma maneira construtiva para se obter algoritmos simples e não sofisticados que convertem a representação canônica de um operador para tal representação compacta.

Considerando os $W$-operadores que são anti-extensivos e idempotentes em um sentido estrito, obtivemos uma simplificação para a sua representação compacta. Além disso, colocando a hipótese de que os $W$-operadores são também crescentes, obtivemos uma realização minimal da representação clássica de Matheron para as aberturas e fechamentos IT's a partir da representação compacta simplificada.

\subsubsection{Decomposição Seqüencial de $W$-Operadores}

Vimos que a sup-decomposição de um operador tem uma estrutura puramente paralela que não é eficiente para implementação em máquinas convencionais seqüenciais. Assim, no Capítulo 9, estudamos e formalizamos o problema de transformar as sup-decomposições em decomposições puramente seqüenciais (quando elas existem). Uma versão especializada deste problema é o problema clássico de encontrar decomposições seqüenciais para erosões e dilatações.

Propomos uma teoria que consiste na formulação e solução de equações discretas em reticulados completos, cujo espaço de soluções tem uma forte natureza combinatória. Essas técnicas foram desenvolvidas para $W$-operadores em geral, depois, especializadas para $W$-operadores crescentes e, posteriormente, aplicadas sobre operadores que podem ser construídos a partir de composições de dilatações e erosões.

Os resultados obtidos para $W$-operadores em geral são uma extensão de alguns resultados obtidos por Jones [27] para operadores crescentes IT's e introduzem alguns novos limites superiores e inferiores para o caso particular de $W$-operadores crescentes. 


\section{Capítulo 4}

\section{Decomposição de Elementos Estruturantes}

Neste capítulo serão dados os fundamentos matemáticos que foram estudados na nossa pesquisa para se obter uma decomposição de um dado EE. A maioria das idéias apresentadas neste capítulo são originais e servem de apoio para os Capítulos 5, 6 e 7.

$\mathrm{Na}$ Seção 4.1, veremos que encontrar uma decomposição de um EE $A$ equivale a procurar uma subseqüência de uma dada seqüência construída a partir de $A$. Na Seção 4.2, apresentaremos algumas propriedades do fecho convexo. $\mathrm{Na}$ Seção 4.3, introduziremos algumas medidas feitas sobre EE's e apresentaremos um limite inferior para o número de subconjuntos para uma decomposição de um EE.

\subsection{Propriedades de uma Decomposição de um Ele- mento Estruturante}

Nesta seção, estabeleceremos uma equivalência entre o problema de encontrar uma decomposição de um EE $A$ e o problema de encontrar uma subseqüência dentro de uma dada seqüência construída a partir de $A$. Para isso, devemos antes apresentar algumas definições e propriedades da adição de Minkowski.

Dado um EE $A$, uma seqüência de subconjuntos de $A$ é uma sucessão de subconjuntos de $A$ em uma determinada ordem pré-estabelecida. Por exemplo, se $B_{1}, B_{2}, B_{3}$, $B_{4}, B_{5}, B_{6}, B_{7}$ são subconjuntos distintos de $A$, então $\left[B_{7}, B_{1}, B_{1}, B_{1}, B_{2}, B_{2}, B_{3}, B_{1}, B_{4}\right.$, $B_{5}, B_{2}, B_{6}$ ] é uma seqüencia de subconjuntos de $A$. Nesta tese, consideraremos somente seqüências de subconjuntos finitas.

Sejam $\mathrm{R}=\left[R_{1}, R_{2}, \cdots, R_{m}\right]$ e $\mathrm{S}=\left[S_{1}, S_{2}, \cdots, S_{n}\right]$ duas seqüências de subconjuntos de um dado EE $A$. Dizemos que R é uma subseqüência de $\mathrm{S}$ se, e somente se, para todo $R_{j} \in \mathrm{R}$, existe $S_{\pi(j)} \in \mathrm{S}$, tal que $R_{j}=S_{\pi(j)}$ (isto é, $\mathrm{R}=\left[S_{\pi(1)}, S_{\pi(2)}, \cdots, S_{\pi(m)}\right]$ ), onde 
$\pi(j)$ é um índice em $\{1,2,3, \cdots, n\}$ e $\pi(1)<\pi(2)<\pi(3)<\cdots<\pi(m)$. Por exemplo, se $B_{1}, B_{2}, B_{3}, B_{4}, B_{5}, B_{6}$ são subconjuntos distintos de $A$, então $\left[B_{1}, B_{1}, B_{4}, B_{2}\right]$ é uma subseqüência de $\left[B_{6}, B_{1}, B_{1}, B_{1}, B_{2}, B_{2}, B_{3}, B_{1}, B_{4}, B_{5}, B_{2}\right]$, mas $\left[B_{1}, B_{1}, B_{4}, B_{1}\right]$ não é.

Dado um EE $A$ e duas seqüências de subconjuntos de $A$, digamos $\mathrm{S}$ e $\mathrm{R}$, a concatenação das seqüências $\mathrm{S}$ e $\mathrm{R}$, denotada por $\mathrm{S} \cdot \mathrm{R}$, é a seqüência formada pelos elementos de $\mathrm{S}$ seguida pelos elementos de R. Por exemplo, se $\mathrm{S}=\left[B_{1}, B_{2}\right]$ e $\mathrm{R}=\left[B_{1}, B_{3}\right]$ são duas seqüências de subconjuntos de $A$, então a concatenação $\mathrm{T}=\mathrm{S} \cdot \mathrm{R}$ é a seqüência $\mathrm{T}=\left[B_{1}, B_{2}, B_{1}, B_{3}\right]$.

Dizemos que um EE $A$ tem uma decomposição seqüencial (ou $A$ é decomponível) se existe uma seqüência $\left[B_{1}, B_{2}, \cdots, B_{n}\right]$ de subconjuntos do quadrado elementar tal que $A=B_{1} \oplus B_{2} \oplus \cdots \oplus B_{n}$. A seqüência $\left[B_{1}, B_{2}, \cdots, B_{n}\right]$ é chamada uma decomposição seqüencial de $A$.

Uma decomposição seqüencial de um EE pode ser particionada em duas subseqüências: de forma e de translação. A subseqüência de forma representa a forma do $\mathrm{EE}$ e ela é formada por subconjuntos que têm pelo menos dois elementos. A subseqüencia de translação define a posição do $E E$ em $\mathbb{Z}^{2}$ e ela é formada por subconjuntos unitários. A subseqüência de forma $\left[B_{1}, \cdots, B_{k}\right]$ é chamada de decomposição de forma (ou simplesmente, decomposição) de $A$ e o número inteiro $k$ é o comprimento de uma decomposição de $A$. Dizemos que uma decomposição de $A$ é ótima se, e somente se, $k$ é o menor valor possível para uma decomposição de $A$.

O resultado seguinte é uma conseqüência imediata da Proposição 3.15.

Proposição 4.1 Seja $A$ um EE. Se $\left[B_{1}, B_{2}, \cdots, B_{k}\right]$ é uma decomposição de $A$, então cada $B_{i}$ é um invariante de $A$.

Prova: Uma vez que $\left[B_{1}, B_{2}, \cdots, B_{k}\right]$ é uma decomposição de $A$, então existe $h \in \mathbb{Z}^{2}$ tal que $A=\left(B_{1} \oplus B_{2} \oplus \cdots \oplus B_{k}\right)_{h}$ ou $A=\left(B_{1} \oplus B_{2} \oplus \cdots \oplus B_{k}\right) \oplus\{h\}$. Assim, pela propriedade de comutatividade e associatividade da adição de Minkowski e pela Proposição 3.15, cada $B_{i}(i=1,2, \cdots, k)$ é um invariante de $A$.

Sejam $A$ e $X$ dois EE's tais que $X$ é um invariante de $A$. A multiplicidade de $X$ com respeito a $A$ é o maior inteiro positivo $n$ tal que $n X$ é ainda um invariante de $A$. Por exemplo, a multiplicidade de $B_{1}, B_{2}$ e $B_{3}$, os conjuntos apresentados na Figura 3.1.b, com respeito a $A$, o conjuntos apresentado na Figura 3.1.a, é 1, uma vez que, para todo $i \in\{1,2,3\}, 2 B_{i}$ não é um invariante de $A$. Observe que conjuntos unitários têm multiplicidade infinita com respeito a qualquer EE $A$.

Introduziremos agora uma relação de equivalência sobre uma coleção genérica $\mathcal{X}$ de subconjuntos de $\mathbb{Z}^{2}$ (isto é, $\mathcal{X} \in \mathcal{P}\left(\mathbb{Z}^{2}\right)$ ). Sejam $X$ e $Y$ dois elementos de $\mathcal{X}$. Dizemos que $X$ e $Y$ são equivalentes sob translação se, e somente se, um pode ser obtido a partir de uma translação do outro, isto é, $X \equiv Y$ se, e somente se, existe $h \in \mathbb{Z}^{2}$ tal que $X_{h}=Y$.

Uma vez que a equivalência sob translação é uma relação de equivalência, o conjunto 
de suas classes de equivalência (isto é, os conjuntos compostos exatamente por todos elementos equivalentes em $\mathcal{X}$ ) constitui uma partição de $\mathcal{X}$.

Denotaremos por $\mathcal{Q}(\mathcal{X})$ o conjunto de todas as classes de equivalência (sob translação) em $\mathcal{X}$ (isto é, o conjunto quociente de $\mathcal{X}$ ). Denotaremos por $\mathcal{R}(\mathcal{X})$ o conjunto composto por exatamente um representante de cada classe de equivalência em $\mathcal{Q}(\mathcal{X})$, isto é, $\mathcal{R}(\mathcal{X})$ é o conjunto tal que $|\mathcal{R}(\mathcal{X})|=|\mathcal{Q}(\mathcal{X})|$.

O conjunto de todos os subconjuntos do quadrado elementar que têm pelo menos dois pontos é denotado por $\mathcal{E}=\left\{B \subseteq\{-1,0,1\}^{2}:|B| \geq 2\right\}$. Denotaremos por SeqQ $=$ $\left[B_{1}, B_{2}, \cdots, B_{n}\right]$ a seqüência formada por todos elementos de $\mathcal{R}(\mathcal{E})$ em uma determinada ordem.

Dado um EE $A$, o conjunto de todos elementos de $\mathcal{R}(\mathcal{E})$ (ou equivalentemente, de todos elementos da seqüência SeqQ) que são invariantes de $A$ é denotado por $\mathcal{B}(A)=\{B \in$ $\mathcal{R}(\mathcal{E}): B$ é um invariante de $A\}$. Por exemplo, o conjunto $\mathcal{B}(A)$ para o EE $A$ apresentado na Figura 3.1.a é $\mathcal{B}(A)=\left\{B_{1}, B_{2}, B_{3}\right\}$, onde $B_{1}, B_{2}$ e $B_{3}$ são os EE's apresentados na Figura 3.1.b.

Dado um EE $A$, a seqüência composta por todos elementos de $\mathcal{B}(A)$ que é uma subseqüência de $\mathrm{SeqQ}=\left[B_{1}, B_{2}, \cdots, B_{n}\right]$ é denotada por SeqB $[A]$. Por exemplo, considere os EE's apresentados na Figura 3.1.b. Se $\left[B_{1}, B_{2}, B_{3}\right]$ é uma subseqüência de SeqQ, então a seqüência SeqB $[A]$ para o $\mathrm{EE} A$ apresentado na Figura 3.1.a é SeqB $[A]=\left[B_{1}, B_{2}, B_{3}\right]$. Neste exemplo, note que $\left[B_{1}, B_{3}, B_{2}\right]$ não é uma subseqüência de SeqQ, e portanto $\operatorname{SeqB}[A] \neq$ $\left[B_{1}, B_{3}, B_{2}\right]$.

Proposição 4.2 Sejam $A$ um EE e $X$ um elemento da seqüência SeqB $[A]$. Se $n$ é a multiplicidade de $X$ com respeito a $A$, então qualquer decomposição de $A$ contém no máximo $n$ elementos iguais a $X$.

Prova: Suponha que exista uma decomposição seqüêncial de $A$ que contenha $m>n$ elementos iguais a $X$, isto é, $A=m X \oplus B_{1} \oplus B_{2} \oplus \cdots \oplus B_{k}$. Pela Proposição 3.15, $m X$ é um invariante de $A$. Mas isto contradiz a definição de multiplicidade de $A$. Logo, qualquer decomposição de $A$ contém no máximo $n$ elementos iguais a $X$.

Seja $X$ um EE e $n$ um inteiro não negativo. Se $n \neq 0$, então a seqüência formada pela sucessão de $n$ conjuntos $X$ é denotada por $\operatorname{Seq}[X, n]$, ou seja, $\operatorname{Seq}[X, n]=[X, X, \cdots, X]$. Se $n=0$, Seq $[X, 0]$ denota a seqüência vazia.

Seja $A$ um EE. Seja SeqB $[A]=\left[B_{1}, B_{2}, \cdots, B_{k}\right]$ e seja $n_{i}$ a multiplicidade de $B_{i}$ com respeito a $A(i=1, \cdots, k)$. A seqüência de invariantes de $A$ é a seqüência $\operatorname{Seq} \operatorname{lnv}[A]=$ $\operatorname{Seq}\left[B_{1}, n_{1}\right] \cdot \operatorname{Seq}\left[B_{2}, n_{2}\right] \cdots \operatorname{Seq}\left[B_{k}, n_{k}\right]$. Por exemplo, a seqüência $\left[B_{1}, B_{2}, B_{3}\right]$ (conjuntos apresentados na Figura 3.1.b) é a seqüencia de invariantes do EE $A$ apresentado na Figura 3.1.a, uma vez que, para $i \in\{1,2,3\}$, a multiplicidade de cada conjunto $B_{i}$ é 1 .

O teorema seguinte é uma conseqüência imediata das Proposições 4.1 e 4.2 . 
Teorema 4.3 Se A é um EE, então A tem uma decomposição seqüêncial se, e somente se, existe uma subseqüência de Seqlnv $[A]$ que é uma decomposição de $A$.

Prova: $(\Rightarrow)$ Seja $\left[B_{1}, B_{2}, \cdots, B_{n}\right]$ uma decomposição seqüencial de $A$. Seja $\left[D_{1}, D_{2}, \cdots\right.$, $\left.D_{k}\right]$ a subseqüência de $\left[B_{1}, B_{2}, \cdots, B_{n}\right]$ que é a decomposição de $A$. Pela Proposição 4.1, cada $D_{i}$ é um invariante de $A$, e, uma vez que todo $D_{i}$ tem pelo menos dois pontos, então, para cada $i \in\{1,2, \cdots, k\}$, existe $X_{r_{i}} \in \operatorname{SeqB}[A]$ tal que $X_{r_{i}} \equiv D_{i}$. Sem perda de generalidade, podemos supor que $r_{i}=i$, para $i \in\{1,2, \cdots, k\}$. Claramente, a seqüência $\left[X_{1}, X_{2}, \cdots, X_{k}\right]$ é uma decomposição de $A$.

Por construção de Seqlnv[A], qualquer elemento de SeqB $[A]$ aparece em Seqlnv $[A]$. Assim, todo elemento distinto da seqüência $\left[X_{1}, X_{2}, \cdots, X_{k}\right]$ aparece em Seqlnv $[A]$. Sejam $Y_{1}, Y_{2}, \cdots, Y_{\ell}$ todos elementos distintos de $\left[X_{1}, X_{2}, \cdots, X_{k}\right]$ tais que, para todo $j \in$ $\{1,2, \cdots, \ell-1\}, Y_{j}$ aparece antes que $Y_{j+1}$ em Seqlnv $[A]$. Para todo $j \in\{1,2, \cdots, \ell\}$, seja $m_{j}$ o número de ocorrências de $Y_{j}$ em $\left[X_{1}, X_{2}, \cdots, X_{k}\right]$. Evidentemente, a seqüência Seq $\left[Y_{1}, m_{1}\right] \cdots \operatorname{Seq}\left[Y_{\ell}, m_{\ell}\right]$ é uma decomposição de $A$.

Note que cada $Y_{j}$ em Seq $\left[Y_{1}, m_{1}\right] \cdots \operatorname{Seq}\left[Y_{\ell}, m_{\ell}\right]$ é um elemento de Seqlnv $[A]$ e, para todo $j \in\{1,2, \cdots, \ell-1\}, Y_{j}$ aparece antes que $Y_{j+1}$ em Seqlnv $[A]$. Assim, para provar que a seqüência Seq $\left[Y_{1}, m_{1}\right] \cdots \operatorname{Seq}\left[Y_{\ell}, m_{\ell}\right]$ é uma subseqüência de Seqlnv $[A]$, temos que mostrar que $m_{j} \leq n_{j}$, onde $n_{j}$ é o número de ocorrências de $Y_{j}$ em Seqlnv[A]. De fato, pela construção de Seqlnv $[A], n_{j}$ é a multiplicidade de $Y_{j}$ com respeito a $A$, assim, pela Proposição 4.2 , qualquer decomposição seqüêncial de $A$ contém no máximo $n_{j}$ elementos iguais a $Y_{j}$, e, portanto, $m_{j} \leq n_{j}$.

$(\Leftarrow)$ Claramente, se existe uma subseqüência de Seqlnv $[A]$ que é uma decomposição de $A$, então $A$ tem uma decomposição seqüencial.

\subsection{Propriedades do Fecho Convexo}

Nesta seção apresentaremos algumas propriedades básicas (Lemas 4.4 a 4.9) do fecho convexo que serão utilizadas nesta tese.

Lema 4.4 Se $A, B \subseteq \mathbb{Z}^{2}$, então

(a) $A \subseteq C(A)$,

(b) $A \subseteq B \Rightarrow C(A) \subseteq C(B)$,

(c) $(C(A))_{h}=C\left(A_{h}\right)$, para todo $h \in \mathbb{Z}^{2}$.

Prova: Sejam $\mathcal{A}$ e $\mathcal{B}$ o conjunto de todos os semiplanos em $\mathbb{Z}^{2}$ que contém, respectivamente, $A$ e $B$. 
Uma vez que $C(A)$ é a intersecção de todos os semiplanos que contém $A$, então, pela propriedade da intersecção dada pela Proposição 2.3.i, $C(A) \subseteq A$. Isto prova $(a)$.

Para provar (b), seja $X \in \mathcal{B}$. Uma vez que $X \supseteq B \supseteq A$, então $X \in \mathcal{A}$. Assim, $\mathcal{B} \subseteq \mathcal{A}$. Desta forma,

$$
\begin{aligned}
\left(\bigcap_{Y \in \mathcal{B}} Y\right) \cap\left(\bigcap_{X \in \mathcal{A}} X\right)=\bigcap_{X \in \mathcal{A}} X & \Leftrightarrow C(B) \cap C(A)=C(A) \\
& \Leftrightarrow C(A) \subseteq C(B) \quad \text { (pela Proposição 2.3.c) }
\end{aligned}
$$

Finalmente, para provar $(c)$, seja $h \in \mathbb{Z}^{2}$ e seja $\mathcal{H}$ o conjunto de todos os semiplanos que contêm $A_{h}$. Assim, $H \in \mathcal{H} \Leftrightarrow H \supseteq A_{h} \Leftrightarrow H_{-h} \supseteq A \Leftrightarrow H_{-h} \in \mathcal{A}$. Logo,

$$
(C(A))_{h}=\left(\bigcap_{H \in \mathcal{A}} H\right)_{h}=\left(\bigcap_{H \in \mathcal{A}} H_{h}\right)=\left(\bigcap_{H_{-h} \in \mathcal{A}} H\right)=\left(\bigcap_{H \in \mathcal{H}} H\right)=C\left(A_{h}\right) .
$$

Lema 4.5 Se $A, B \subseteq \mathbb{Z}^{2}$, então
(a) $C(A) \cup C(B) \subseteq C(A \cup B)$,
(b) $C(A) \cap C(B) \supseteq C(A \cap B)$.

Prova: Vamos provar (a). Pela Proposição 2.3.b, temos que $A \subseteq A \cup B$ e $B \subseteq A \cup B$. Assim, pelo Lema 4.4.b, $C(A) \subseteq C(A \cup B)$ e $C(B) \subseteq C(A \cup B)$. Logo, pela Proposição 2.3.j, $C(A) \cup C(B) \subseteq C(A \cup B)$. De maneira similar pode-se demonstrar a Propriedade $(b)$.

Lema 4.6 Se $A \subseteq \mathbb{Z}^{2}$, então $C(C(A))=C(A)$.

Prova: Por um lado, pelos Lemas 4.4.a e 4.4.b, é fácil ver que $C(A) \subseteq C(C(A))$. Por outro lado, $C(C(A)) \subseteq C(A)$. De fato, seja $\mathcal{A}$ o conjunto de todos semiplanos que contêm A. Logo,

$$
\begin{array}{rlr}
C(C(A)) & =C\left(\bigcap_{X \in \mathcal{A}} X\right) & \\
& \subseteq \bigcap_{X \in \mathcal{A}} C(X) & \text { (pelo Lema 4.5.b) } \\
& =\bigcap_{X \in \mathcal{A}} X \quad(\text { uma vez que } C(X)=X, \text { para todo } X \in \mathcal{A}) \\
& =C(A) .
\end{array}
$$

Portanto, $C(C(A))=C(A)$.

Lema 4.7 Se $A, B \subseteq \mathbb{Z}^{2}$, então $C(A) \oplus B \subseteq C(A \oplus B)$.

\section{Prova:}




$$
\begin{array}{rlrl}
C(A) \oplus B & =\bigcup_{b \in B}(C(A))_{b} & \\
& =\bigcup_{b \in B} C\left(A_{b}\right) & & \text { (pelo Lema 4.4.c) } \\
& \subseteq C\left(\bigcup_{b \in B} A_{b}\right) & & \text { (pelo Lema 4.j.a) } \\
& =C(A \oplus B) . &
\end{array}
$$

Lema 4.8 Se $A, B \subseteq \mathbb{Z}^{2}$, então $C(A \oplus C(B)) \subseteq C(A \oplus B)$.

Prova: Pelo Lema 4.7, $A \oplus C(B)=C(B) \oplus A \subseteq C(A \oplus B)$. Pelos Lemas 4.4.b e 4.6, $C(A \oplus C(B)) \subseteq C(C(A \oplus B))=C(A \oplus B)$.

Lema 4.9 Se $A, B \subseteq \mathbb{Z}^{2}$, então $C(C(A) \oplus C(B))=C(A \oplus B)$.

Prova: Primeiro, vamos provar que $C(A \oplus B) \subseteq C(C(A) \oplus C(B))$. Pelo Lema 4.4.a, $A \subseteq C(A)$ e $B \subseteq C(B)$. Então, pela Proposição 3.8.g, $A \oplus B \subseteq C(A) \oplus B$ e, pela Proposição 3.8.d, $C(A) \oplus B \subseteq C(A) \oplus C(B)$. Logo, $A \oplus B \subseteq \bar{C}(A) \oplus C(B)$ e, pelo Lema 4.4.b, $C(A \oplus B) \subseteq C(C(A) \oplus C(B))$.

Finalmente, provaremos que $C(C(A) \oplus C(B)) \subseteq C(A \oplus B)$. Pelo Lema 4.7, $C(A) \oplus$ $C(B) \subseteq C(A \oplus C(B))$. Assim,

$$
\begin{aligned}
C(C(A) \oplus C(B)) & \subseteq C(C(A \oplus C(B))) & & \text { (pelo Lema 4.4.b) } \\
& =C(A \oplus C(B)) & & \text { (pelo Lema 4.6) } \\
& \subseteq C(A \oplus B) & & \text { (pelo Lema 4.8). }
\end{aligned}
$$

Portanto, $C(C(A) \oplus C(B))=C(A \oplus B)$.

\subsection{Algumas Medidas Tomadas sobre um Elemento Estruturante}

Nesta seção, definiremos duas medidas (Subseções 4.3.2 e 4.3.3) tomadas sobre EE's que serão utilizadas largamente nesta tese. Para isso, precisamos de algumas definições e resultados apresentados na Subseção 4.3.1. Como aplicação dessas medidas, na Subseção 4.3.4, apresentamos um limite inferior para o comprimento de uma decomposição de um EE.

\subsubsection{Lados ou Arestas de um Elemento Estruturante}

Sejam $\vec{u}_{0}, \vec{u}_{1}, \vec{u}_{2}$ e $\vec{u}_{3}$ os eixos Cartesianos que passam pela origem de $\mathbb{Z}^{2}$ e que tenham, respectivamente, as inclinações, $-90,-45,0$ e 45 graus (veja a Figura 4.1). Para um 


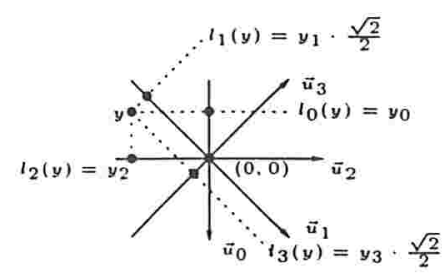

Figura 4.1: Um ponto $y$ e os eixos $\vec{u}_{0}, \vec{u}_{1}, \vec{u}_{2}$ e $\vec{u}_{3}$.

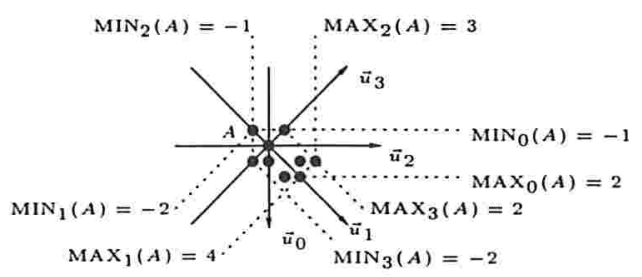

Figura 4.2: Um EE $A$ com os eixos $\vec{u}_{0}, \vec{u}_{1}, \vec{u}_{2}$ e $\vec{u}_{3}$.

dado ponto $x \in \mathbb{Z}^{2}$, sejam $l_{0}(x), l_{1}(x), l_{2}(x)$ e $l_{3}(x)$ as projeções ortogonais de $x$ sobre os eixos Cartesianos $\vec{u}_{0}, \vec{u}_{1}, \vec{u}_{2}$ e $\vec{u}_{3}$, respectivamente. Observe que estas projeções são números inteiros nas direções de -90 e 0 graus e números reais (proporcionais a $\frac{\sqrt{2}}{2}$ ) nas direções de -45 e 45 graus. Dado um ponto $x \in \mathbb{Z}^{2}$, denotaremos por $x_{0}, x_{1}, x_{2}$ e $x_{3}$ as projeções ortogonais normalizadas do ponto $x$ sobre os eixos Cartesianos $\vec{u}_{0}$, $\vec{u}_{1}$, $\vec{u}_{2}$ e $\vec{u}_{3}$, respectivamente, dadas por $x_{0}=l_{0}(x), x_{1}=l_{1}(x) \cdot \sqrt{2}, x_{2}=l_{2}(x)$ e $x_{3}=$ $l_{3}(x) \cdot \sqrt{2}$, respectivamente. Por exemplo, as projeções ortogonais normalizadas do ponto $y=(-5,2) \in \mathbb{Z}^{2}$ são $y_{0}=-2, y_{1}=-7, y_{2}=-5$ e $y_{3}=-3$ (veja a Figura 4.1 ).

Seja $A$ um EE. Para $i=0,1,2,3$, sejam $\operatorname{MAX}_{i}(A)$ e $\operatorname{MIN}_{i}(A)$, respectivamente, as projeções ortogonais normalizadas máxima e mínima sobre o eixo Cartesiano $\vec{u}_{i}$ dos pontos de $A$, isto é, $\operatorname{MAX}_{i}(A)=\max \left\{x_{i}: x \in A\right\}$ e $\operatorname{MIN}_{i}(A)=\min \left\{x_{i}: x \in A\right\}$. Por exemplo, as projeções ortogonais normalizadas máxima e mínima do EE $A$ apresentado na Figura 4.2 são, respectivamente, $\operatorname{MAX}_{0}(A)=2, \operatorname{MAX}_{1}(A)=4, \operatorname{MAX}_{2}(A)=3, \operatorname{MAX}_{3}(A)=2 \mathrm{e}$ $\operatorname{MIN}_{0}(A)=-1, \operatorname{MIN}_{1}(A)=-2, \operatorname{MIN}_{2}(A)=-1, \operatorname{MIN}_{3}(A)=-2$.

Os Lemas de 4.10 a 4.12 fornecem algumas propriedades de $\operatorname{MAX}_{i}(A)$ e $\operatorname{MIN}_{i}(A)$ de um dado $\mathrm{EE} A$.

Lema 4.10 Se $A$ e $B$ são EE's, então, para todo $i \in\{0,1,2,3\}$,

$$
\operatorname{MAX}_{i}(A \oplus B)=\operatorname{MAX}_{i}(A)+\operatorname{MAX}_{i}(B) e \operatorname{MIN}_{i}(A \oplus B)=\operatorname{MiN}_{i}(A)+\operatorname{MiN}_{i}(B) .
$$

Prova: $\operatorname{MAX}_{i}(A \oplus B)=\max \left\{x_{i}: x \in A \oplus B\right\}=\max \left\{a_{i}+b_{i}: a \in A, b \in B\right\}=\max \left\{a_{i}:\right.$ $a \in A\}+\max \left\{b_{i}: b \in B\right\}=\operatorname{MAX}_{i}(A)+\operatorname{MAX}_{i}(B)$. De maneira similar, podemos demonstrar que $\operatorname{MIN}_{i}(A \oplus B)=\operatorname{MIN}_{i}(A)+\operatorname{MIN}_{i}(B)$.

O próximo lema é uma conseqüência imediata do Lema 4.10. 


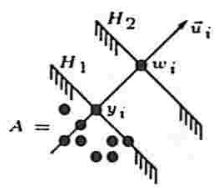

Figura 4.3: Ilustração para a demonstração do Lema 4.12 .

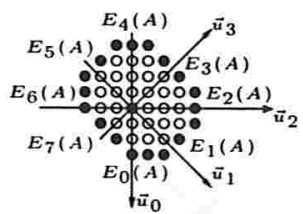

Figura 4.4: Os oito lados de um EE convexo $A$.

Lema 4.11 Se $X$ é um $E E$ e $h$ é um ponto em $\mathbb{Z}^{2}$, então $\operatorname{MAX}_{i}\left(X_{h}\right)=\operatorname{MAX}_{i}(X)+h_{i}$ $e \operatorname{MIN}_{i}\left(X_{h}\right)=\operatorname{MIN}_{i}(X)+h_{i}$.

Prova: Pela Proposição 3.8.b, temos que $X_{h}=X \oplus\{h\}$. Assim, pelo Lema 4.10, $\operatorname{MAX}_{i}\left(X_{h}\right)=\operatorname{MAX}_{i}(X \oplus\{h\})=\operatorname{MAX}_{i}(X)+\operatorname{MAX}_{i}(h)=\operatorname{MAX}_{i}(X)+h_{i}$. Analogamente, $\operatorname{MIN}_{i}\left(X_{h}\right)=\operatorname{MIN}_{i}(X)+h_{i}$

Lema 4.12 Se $A$ é um EE, então, para todo $i \in\{0,1,2,3\}$,

$$
\operatorname{MAX}_{i}(C(A))=\operatorname{MAX}_{i}(A) \text { e } \operatorname{MIN}_{i}(C(A))=\operatorname{MIN}_{i}(A) .
$$

Prova: Para todo $i=0,1,2,3$, pela definição de $\operatorname{MAX}_{i}(A)$, existe $y \in A$ tal que $y_{i}=$ $\operatorname{MAX}_{i}(A)$. Por um lado, $\operatorname{MAX}_{i}(A) \leq \operatorname{MAX}_{i}(C(A))$, uma vez que, pelo Lema 4.4.a, $A \subseteq C(A)$. Por outro lado, $\operatorname{MAX}_{i}(A) \geq \operatorname{MAX}_{i}(C(A))$. De fato, suponha por absurdo que $\operatorname{MAX}_{i}(A)<\operatorname{MAX}_{i}(C(A))$. Então, existe $w \in C(A)$ tal que $\operatorname{MAX}_{i}(A)<w_{i}$. Sejam $H_{1}=\left\{x \in \mathbb{Z}^{2}: x_{i} \leq \operatorname{MAX}_{i}(A)\right\}$ e $H_{2}=\left\{x \in \mathbb{Z}^{2}: x_{i} \leq w_{i}\right\}$. Claramente, $H_{1}$ e $H_{2}$ são dois semiplanos que contém $A$ e $w \notin H_{1}$ (veja a Figura 4.3). Uma vez que $C(A)$ é a intersecção de todos semiplanos que contém $A$, então, $w \notin C(A)$. Mas isto contradiz a escolha de $w \in C(A)$. Portanto, $\operatorname{MAX}_{i}(A) \geq \operatorname{MAX}_{i}(C(A))$. A prova de que $\operatorname{MIN}_{i}(C(A))=\operatorname{MIN}_{i}(A)$ pode ser feita de uma maneira similar.

Seja $A$ um EE. Definimos a seguir os oito lados ou arestas de $A$, denotados por $E_{0}(A), E_{1}(A), E_{2}(A), \cdots, E_{7}(A)$, da seguinte forma. Para $i \in\{0,1,2,3\}, E_{i}(A)$ e $E_{i+4}(A)$ são os conjuntos contendo todos pontos de $C(A)$ que têm, respectivamente, a mesma projeção ortogonal normalizada máxima e mínima sobre o eixo $\vec{u}_{i}$, isto é, $E_{i}(A)=\{x \in$ $\left.C(A): x_{i}=\operatorname{MAX}_{i}(A)\right\}$ e $E_{i+4}(A)=\left\{x \in C(A): x_{i}=\operatorname{MIN}_{i}(A)\right\}$ (veja a Figura 4.4 para um exemplo). Note que o eixo $u_{i}$ é perpendicular aos lados $E_{i}(A)$ e $E_{i+4}(A)$ (veja a Figura 4.4). 


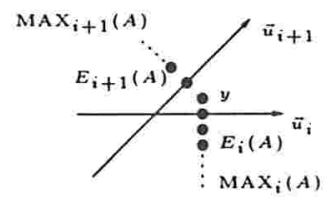

Figura 4.5: Os lados $E_{i}(A)$ e $E_{i+1}(A)$ de um EE $A$.

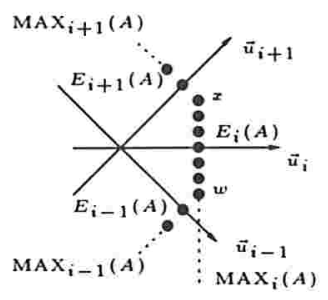

Figura 4.6: Extremidades do lado $E_{i}(A)$.

Pela construção de $E_{i}(A), i \in\{0,1,2, \cdots, 7\}$, e, pelos Lemas 4.6 e 4.12 , é evidente que $E_{i}(A)=E_{i}(C(A)) \subseteq C(A)$.

Dado um EE $A$, o próximo lado de $E_{i}(A)$ é $E_{i+1}(A)$, se $i<7$, ou $E_{0}(A)$, se $i=7$. O lado anterior de $E_{i}(A)$ é $E_{i-1}(A)$, se $i>0$, ou $E_{7}(A)$, se $i=0$. Por exemplo, os próximos lados de $E_{1}(A)$ e $E_{7}(A)$ são, respectivamente, $E_{2}(A)$ e $E_{0}(A)$; os lados anteriores de $E_{5}(A)$ e $E_{0}(A)$ são, respectivamente, $E_{4}(A)$ e $E_{7}(A)$. Por simplicidade de notação, denotaremos $E_{i-1}(A)$ e $E_{i+1}(A)$, respectivamente, o próximo lado e o lado anterior de $E_{i}(A)$.

Lema 4.13 Se $A$ é um $E E$, então, para todo $i \in\{0,1, \cdots, 7\},\left|E_{i}(A) \cap E_{i+1}(A)\right|=1$.

Prova: Suponha que $i=1$ ou 2 (os outros casos, ou seja, para $i=0,3,4,5,6,7$, podem ser provados de uma maneira similar).

Considere o sistema de coordenadas formado pelos eixos Cartesianos $\vec{u}_{i}$ e $\vec{u}_{i+1}$ (veja a Figura 4.5). Neste sistema, qualquer ponto $x \in \mathbb{Z}^{2}$ pode ser unicamente representado por um par ordenado $\left(x_{i}, x_{i+1}\right)$.

Claramente, $E_{i}(A) \cup E_{i+1}(A)$ é um subconjunto 8-conexo de $C(A)$ (veja a Figura 4.5). Assim, pelas definições de $E_{i}(A)$ e $E_{i+1}(A)$, existe um ponto $y \in E_{i}(A) \cup E_{i+1}(A)$ tal que $y_{i}=\operatorname{MAX}_{i}(A)$ e $y_{i+1}=\operatorname{MAX}_{i+1}(A)$ (veja Figura 4.5). Assim, também pelas definições de $E_{i}(A)$ e $E_{i+1}(A), y \in E_{i}(A)$ e $y \in E_{i+1}(A)$. Resta-nos mostrar que este ponto é único. Suponha que existam dois pontos $y, z \in E_{i}(A) \cap E_{i+1}(A)$. Neste caso, $y_{i}=z_{i}$ (uma vez que $\left.y, z \in E_{i}(A)\right)$ e $y_{i+1}=z_{i+1}$ (uma vez que $y, z \in E_{i+1}(A)$ ). Assim, $y=\left(y_{i}, y_{i+1}\right)=$ $\left(z_{i}, z_{i+1}\right)=z \mathrm{e}$, portanto, $\left|E_{i}(A) \cap E_{i+1}(A)\right|=1$.

Dado um EE $A$, é fácil ver que, para todo $i \in\{0,1 . \cdots, 7\}, E_{i}(A)$ é uma linha formada por pontos consecutivos de $C(A)$ nas direções com inclinações $-90,-45,0$ ou 45 graus. 
Assim, cada $E_{i}(A)$ contém no máximo dois pontos que chamaremos extremidades de $E_{i}(A)$. Mais formalmente, as duas extremidades de $E_{i}(A)$ são os pontos $x, y \in E_{i}(A)$ tais que $x \in E_{i+1}(A)$ e $y \in E_{i-1}(A)$. Por exemplo, na Figura 4.6, os pontos $x$ e $w$ são as duas extremidades de $E_{i}(A)$.

Lema 4.14 Se $A$ e $B$ são EE's, então, para todo $i \in\{0,1, \cdots, 7\}, E_{i}(A \oplus B)=E_{i}(A) \oplus$ $E_{i}(B)$.

Prova: Suponha que $i=1$ ou 2 (os outros casos, ou seja, para $i=0,3,4,5,6,7$, podem ser demonstrados de uma maneira similar).

Primeiro, vamos provar que $E_{i}(A) \oplus E_{i}(B) \subseteq E_{i}(A \oplus B)$.

Pela definição de $E_{i}(A \oplus B), x \in E_{i}(A \oplus B)$ se, e somente se, $x \in C(A \oplus B)$ e $x_{i}=$ $\operatorname{MAX}_{i}(A \oplus B)$. Assim, para provar que $E_{i}(A) \oplus E_{i}(B) \subseteq E_{i}(A \oplus B)$, devemos mostrar que, se $x \in E_{i}(A) \oplus E_{i}(B)$, então $x \in C(A \oplus B)$ e $x_{i}=\operatorname{MAX}_{i}(A \oplus B)=\operatorname{MAX}_{i}(A)+\operatorname{MAX}_{i}(B)$ (pelo Lemma 4.10).

Uma vez que $E_{i}(A) \subseteq C(A)$ e $E_{i}(B) \subseteq C(B)$, então, pela Proposição 3.8.d, $E_{i}(A) \oplus$ $E_{i}(B) \subseteq E_{i}(A) \oplus C(B) \subseteq C(A) \oplus C(B)$. Assim, pelos Lemas 4.4.a e 4.9, $E_{i}(A) \oplus$ $E_{i}(B) \subseteq C(A \oplus B)$. Resta-nos mostrar que $x_{i}=\operatorname{MAX}_{i}(A)+\operatorname{MAX}_{i}(B)$. Pela definição da adição de Minkowski, se $x \in E_{i}(A) \oplus E_{i}(B)$, então existem $y \in E_{i}(A)$ e $z \in E_{i}(B)$ tais que $x=y+z$. Pela definição de $E_{i}(A), y \in E_{i}(A)$ se, e somente se, $y \in C(A)$ e $y_{i}=\operatorname{MAX}_{i}(A)$. Analogamente, $z \in E_{i}(B)$ se, e somente se, $z \in C(B)$ e $z_{i}=\operatorname{MAX}_{i}(B)$. Assim, uma vez que $x=y+z$, então $x_{i}=y_{i}+z_{i}=\operatorname{MAX}_{i}(A)+\operatorname{MAX}_{i}(B)$. Logo, $E_{i}(A) \oplus E_{i}(B) \subseteq E_{i}(A \oplus B)$.

Agora, provaremos que $E_{i}(A \oplus B) \subseteq E_{i}(A) \oplus E_{i}(B)$.

Por um lado, por definição, os lados $E_{i}(A)$ e $E_{i}(B)$ são linhas formadas por pontos consecutivos de $C(A)$ e $C(B)$, respectivamente, em uma mesma direção com inclinação de -45 ou 0 graus (uma vez que $i=1$ ou 2). Além disso, o eixo Cartesiano $u_{i}$ é perpendicular a $E_{i}(A)$ e $E_{i}(B)$. Então, pela definição da adição de Minkowski, $E_{i}(A) \oplus E_{i}(B)$ é uma linha formada por um segmento de pontos consecutivos de $C(A) \oplus C(B)$ na mesma direção que $E_{i}(A)$ e $E_{i}(B)$. Assim, o eixo Cartesiano $u_{i}$ é também perpendicular a $E_{i}(A) \oplus E_{i}(B)$.

Por outro lado, por definição, $E_{i}(A \oplus B)$ é uma linha formada por um segmento consecutivo de pontos de $C(A \oplus B)$ na mesma direção que $E_{i}(A)$ e $E_{i}(B)$. Dessa forma, o eixo Cartesiano $u_{i}$ é também perpendicular a $E_{i}(A \oplus B)$.

Assim, se as extremidades de $E_{i}(A \oplus B)$ pertencem a $E_{i}(A) \oplus E_{i}(B)$, então, obviamente, $E_{i}(A \oplus B) \subseteq E_{i}(A) \oplus E_{i}(B)$.

Sejam $t$ e $z$ as extremidades de $E_{i}(A \oplus B)$ tais que $\{t\}=E_{i-1}(A \oplus B) \cap E_{i}(A \oplus B)$ e $\{z\}=E_{i}(A \oplus B) \cap E_{i+1}(A \oplus B)$. Vamos mostrar que $z, t \in E_{i}(A) \oplus E_{i}(B)$. Para isso, sejam $x$ e $y$ extremidades de, respectivamente, $E_{i}(A)$ e $E_{i}(B)$ tais que $\{x\}=E_{i}(A) \cap E_{i+1}(A)$ e $\{y\}=E_{i}(B) \cap E_{i+1}(B)$. 


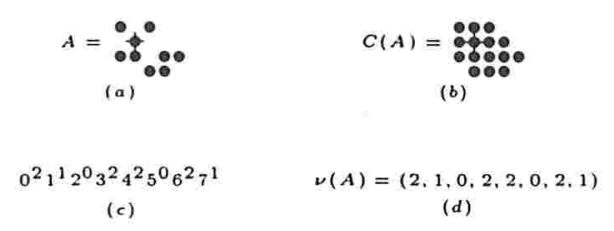

Figura 4.7: (a) Um EE $A$. (b) O fecho convexo de $A$. (c) O código de cadeia de $C(A)$. (d) $\mathrm{O}$ vetor projeção de $A$.

No sistema de coordenadas formado pelos eixos Cartesianos $\vec{u}_{i}$ e $\vec{u}_{i+1}$, temos que $x=\left(x_{i}, x_{i+1}\right), y=\left(y_{i}, y_{i+1}\right)$ e $z=\left(z_{i}, z_{i+1}\right)$.

Uma vez que $x \in E_{i}(A), y \in E_{i}(B), z \in E_{i}(A \oplus B)$ e $i=1$ ou 2, então, $x_{i}=\operatorname{MAX}_{i}(A)$, $y_{i}=\operatorname{MAX}_{i}(B)$ e $z_{i}=\operatorname{MAX}_{i}(A \oplus B)$. Assim, pelo Lema 4.10, $z_{i}=\operatorname{MAX}_{i}(A \oplus B)=$ $\operatorname{MAX}_{i}(A)+\operatorname{MAX}_{i}(B)=x_{i}+y_{i}$. Analogamente, $z_{i+1}=x_{i+1}+y_{i+1}$. Logo, $z=\left(z_{i}, z_{i+1}\right)=$ $\left(x_{i}, x_{i+1}\right)+\left(y_{i}, y_{i+1}\right)=x+y$.

De maneira similar, se $r$ e $s$ são, respectivamente, extremidades de $E_{i}(A)$ e $E_{i}(B)$ tais que $\{r\}=E_{i-1}(A) \cap E_{i}(A)$ e $\{s\}=E_{i-1}(B) \cap E_{i}(B)$, então $t=r+s$.

Portanto, uma vez que $E_{i}(A) \oplus E_{i}(B)=\left\{u+v: u \in E_{i}(A), v \in E_{i}(B)\right\}$, então $z=x+y$ e $t=r+s$ pertencem a $E_{i}(A) \oplus E_{i}(B)$.

Lema 4.15 Se A e B são EE's, então, para todo $i \in\{0,1, \cdots, 7\},\left|E_{i}(A \oplus B)\right|=\left|E_{i}(A)\right|+$ $\left|E_{i}(B)\right|-1$.

Prova: Uma vez que $E_{i}(A)$ e $E_{i}(B)$ são linhas formadas por segmentos de pontos consecutivos, respectivamente, de $C(A)$ e $C(B)$, ambas com uma mesma direção de inclinação -90, $-45,0$ ou 45 graus, então, pela definição da adição de Minkowski, é fácil ver que a cardinalidade de $E_{i}(A) \oplus E_{i}(B)$ é igual a $\left|E_{i}(A)\right|+\left|E_{i}(B)\right|-1$. Portanto, $\left|E_{i}(A \oplus B)\right|=$ $\left|E_{i}(A)\right|+\left|E_{i}(B)\right|-1$, uma vez que, pelo Lema 4.14, $E_{i}(A \oplus B)=E_{i}(A) \oplus E_{i}(B)$.

\subsubsection{Vetor Projeção de um Elemento Estruturante}

O vetor projeção de um dado EE $A$ é o vetor $\nu(A)=\left(\nu_{0}(A), \nu_{1}(A), \cdots, \nu_{7}(A)\right) \in \mathbb{Z}^{8}$ tal que cada uma de suas coordenadas corresponde ao valor $\left|E_{i}(A)\right|-1$ (veja as Figuras 4.7.a, 4.7.b e 4.7.d) Mais formalmente, o vetor projeção do $\operatorname{EE} A$ é $\nu(A)=\left(\nu_{0}(A), \nu_{1}(A), \cdots, \nu_{7}(A)\right)$, onde $\nu_{i}(A)=\left|E_{i}(A)\right|-1$.

Note que o vetor projeção é independente de translação, ou seja, $\nu(A)=\nu\left(A_{h}\right)$, para todo $h \in \mathbb{Z}^{2}$. Observe também que, para todo EE $A, \nu(A)=\nu(C(A))$, uma vez que, por definição, $E_{i}(A)=E_{i}(C(A))$. 


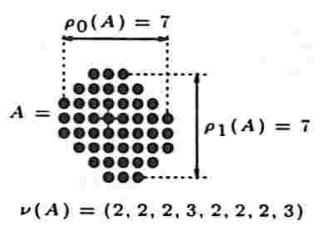

Figura 4.8: $O$ vetor retangular de $A$.

Kanungo e Haralick [28] estudaram algumas propriedades de decomposição de EE's convexos e 4-conexos, onde usaram um esquema de codificação de borda muito similar com o vetor projeção definido acima.

Sabe-se que o código de cadeia ("chain code") [18][22, p. 484] descreve completamente a forma de um EE convexo. Sem perda de generalidade, assumiremos que o código de cadeia de um EE convexo começa na direção de 0 graus e percorre o EE em sentido anti-horário. Assim, o código de cadeia de um EE convexo pode ser representado por uma seqüência de números entre 0 e $7: 0^{n_{0}} 1^{n_{1}} \cdots 7^{n_{7}}$, onde $i^{n_{i}}\left(n_{i} \geq 0\right)$ é a seqüência de números $i$ definida da seguinte forma: $i$ repete $n_{i}$ vezes se $n_{i}>0$ ou $i^{n_{i}}$ é uma sequência vazia se $n_{i}=0$. Por exemplo, o código de cadeia do EE apresentado na Figura 4.7.b é mostrado na in Figura 4.7.c. Podemos facilmente ver que o vetor projeção de um dado EE $A$ e o código de cadeia de $C(A)$ são definições equivalentes (veja as Figuras 4.T.c e 4.7.d).

Dado um EE convexo $A$, um algoritmo $O(|A|)$ para encontrar o código de cadeia de $A$ (ou equivalentemente, o vetor projeção de $A$ ) pode ser visto em [36, p. 143].

O próximo resultado fornece uma importante propriedade do vetor projeção de um dado EE. O mesmo resultado para EE's convexos que são 4-conexos pode ser encontrado em [28].

Proposição 4.16 Sejam $A, X$ e $Y$ EE's. Se $A \equiv X \oplus Y$, então $\nu(A)=\nu(X)+\nu(Y)$.

Prova: Uma vez que o vetor projeção é independente de translação, então, para todo $i \in\{0,1, \cdots, 7\}$,

$$
\begin{aligned}
\nu_{i}(A) & =\nu_{i}(X \oplus Y) \\
& =\left|E_{i}(X \oplus Y)\right|-1 \\
& =\left(\left|E_{i}(X)\right|+\left|E_{i}(Y)\right|-1\right)-1 \\
& =\left(\left|E_{i}(X)\right|-1\right)+\left(\left|E_{i}(Y)\right|-1\right) \\
& =\nu_{i}(X)+\nu_{i}(Y) .
\end{aligned}
$$

Portanto, $\nu(A)=\nu(X)+\nu(Y)$. 


\subsubsection{Vetor Retangular de um Elemento Estruturante}

$\mathrm{O}$ vetor retangular de um EE $A$ é o vetor $\rho(A)=\left(\rho_{0}(A), \rho_{1}(A)\right) \in \mathbb{Z}^{2}$ tal que, $\rho_{0}(A)=$ $\nu_{3}(A)+\nu_{4}(A)+\nu_{5}(A)$ e $\rho_{1}(A)=\nu_{1}(A)+\nu_{2}(A)+\nu_{3}(A)$. Por exemplo, o vetor retangular do EE $A$ apresentado na Figura 4.8 é $\rho(A)=(7,7)$. Em outras palavras, as coordenadas do vetor retangular de um EE $A$ são os comprimentos dos lados do menor retângulo que contém $A$. Note que, para todo subconjunto $B$ do quadrado elementar, $\rho(B) \leq(2,2)$, isto é, $\rho_{0}(B) \leq 2$ e $\rho_{1}(B) \leq 2$.

Uma vez que o vetor projeção é independente de translação, então o vetor retangular também o é, ou seja, para todo $h \in \mathbb{Z}^{2}, \rho(A)=\rho\left(A_{h}\right)$. Uma vez que, para todo EE $A$, $\nu(A)=\nu(C(A))$, temos que $\rho(A)=\rho(C(A))$.

O próximo resultado é uma conseqüência imediata da definição de vetor retangular e da Proposição 4.16.

Proposição 4.17 Sejam $A, X$ e $Y$ EE's. Se $A \equiv X \oplus Y$, então $\rho(A)=\rho(X)+\rho(Y)$.

\subsubsection{Limite Inferior de uma Decomposição}

O teorema seguinte fornece um limite inferior para o comprimento de uma decomposição de um EE. Um resultado análogo também pode ser encontrado em [49].

Teorema 4.18 Seja A um EE. Se A tem uma decomposição, então uma decomposição de $A$ contém pelo menos lower $(A)=\left\lceil\max \left\{\rho_{0}(A), \rho_{1}(A)\right\} / 2\right\rceil$ elementos.

Prova: Seja $\left[B_{1}, B_{2}, \cdots, B_{m}\right]$ uma decomposição de $A$. Então, $A \equiv B_{1} \oplus B_{2} \oplus \cdots \oplus B_{m}$, e, conseqüentemente, existe $h \in \mathbb{Z}^{2}$ tal que $A=\left(B_{1} \oplus B_{2} \oplus \cdots \oplus B_{m}\right)_{h}$. Para todo $i=$ $1,2, \cdots, m$, seja $S_{i}$ o quadrado $3 \times 3$ que contém $B_{i}$. Claramente, $A \subseteq\left(S_{1} \oplus S_{2} \oplus \cdots \oplus S_{m}\right)_{h}$ e o vetor retangular de $\left(S_{1} \oplus S_{2} \oplus \cdots \oplus S_{m}\right)_{h}$ é $\rho\left((m S)_{h}\right)=\rho(m S)=(2 m, 2 m)$, onde $S$ é o quadrado elementar.

Logo, uma vez que $A \subseteq\left(S_{1} \oplus S_{2} \oplus \cdots \oplus S_{m}\right)_{h}$, então $\rho_{0}(A) \leq 2 m$ e $\rho_{1}(A) \leq 2 m$. Assim, $\max \left\{\rho_{0}(A), \rho_{1}(A)\right\} \leq 2 m$ e, portanto, $m \geq\left\lceil\max \left\{\rho_{0}(A), \rho_{1}(A)\right\} / 2\right\rceil$.

Dado um EE $A$, o comprimento de uma decomposição ótima $A$ deve ser maior ou igual ao limite inferior estabelecido pelo Teorema 4.18. Claramente, se o comprimento de uma decomposição de $A$ é igual a esse limite inferior, então essa decomposição é ótima. No caso de EE's convexos, mostraremos no capítulo seguinte que esse limite inferior é justo.

\subsection{Conclusão}

Neste capítulo, apresentamos algumas propriedades e definições que serão utilizadas nos Capítulos 5, 6 e 7 . 
Vimos que o problema de encontrar uma decomposição de um EE $A$ é equivalente ao problema de encontrar uma subseqüência de uma seqüência de subconjuntos do quadrado elementar construída a partir de $A$. Apresentamos também algumas propriedades básicas do fecho convexo e introduzimos duas importantes medidas sobre um EE: o vetor projeção e o vetor retangular. Finalmente, explorando uma propriedade do vetor retangular, estabelecemos um limite inferior para o comprimento de uma decomposição de um EE. 


\section{Capítulo 5}

\section{Decomposição de Elementos Estruturantes Convexos}

Este capítulo apresenta um algoritmo guloso simples para decompor um EE convexo como seqüência de adições de Minkowski de subconjuntos do quadrado elementar. A técnica proposta está baseada em propriedades algébricas e geométricas da adição de Minkowski. Além da simplicidade do algoritmo, a vantagem desta técnica sobre outros algoritmos conhecidos [49, 34] é que o algoritmo proposto gera uma seqüência mínima de subconjuntos não necessariamente convexos, o que significa que a decomposição contém subconjuntos de menor cardinalidade e, conseqüentemente, em comparação com os algoritmos conhecidos que geram somente subconjuntos convexos, obtemos implementações mais eficientes das correspondentes dilatações e erosões.

Na Seção 5.1 descrevemos suscintamente dois algoritmos conhecidos para decompor EE's convexos. Na Seção 5.2, descrevemos resumidamente o método guloso para resolver problemas de Otimização Combinatória; e na Seção 5.3, apresentamos o algoritmo proposto. Resultados experimentais, prova de corretude e análise computacional do algoritmo estão apresentados, respectivamente, nas Seções 5.4, 5.5 e 5.6. Finalmente, na Seção 5.7, apresentamos uma conclusão deste capítulo.

\subsection{Algoritmos Clássicos para Decompor Elementos Estruturantes Convexos}

Nesta seção serão descritos dois algoritmos clássicos para decompor EE's convexos. Uma restrição forte desses algoritmos é que todos subconjuntos do quadrado elementar que estão na decomposição gerada por esses algoritmos são convexos.

Em 1991, Xu [49] apresentou um algoritmo para decomposição de EE's convexos em termos de um número mínimo de subconjuntos convexos do quadrado elementar. O 
algoritmo do $\mathrm{Xu}$ [49] pode ser dividido em dois principais passos:

Passo 1 : uma decomposição de um EE convexo é encontrada resolvendo-se um sistema de equações lineares com um número fixo de variáveis;

Passo 2 : aplica-se um intricado processo de otimização, subdividido em vários complexos subcasos, para juntar, por adições de Minkowski, os subconjuntos convexos encontrados no Passo 1.

Em 1994, Park e Chin [34] desenvolveram um novo algoritmo mais simples, que é uma extensão do Passo 1 do algoritmo do Xu, para encontrar uma decomposição ótima de EE's convexos. No entanto, o algoritmo deles continua utilizando, como processo de otimização, o Passo 2 do algoritmo do Xu.

Para finalizar esta seção, apresentamos a complexidade de tempo desses algoritmos. Dado um EE convexo $A$, a complexidade de tempo de cada um desses dois algoritmos é $O\left(n^{2}\right)$, onde $n=\max \left\{\rho_{0}(A), \rho_{1}(A)\right\}$. Veremos, neste capítulo, que a complexidade de tempo do algoritmo guloso proposto é $O\left(n^{3}\right)$. A vantagem de nosso algoritmo em relação a estes algoritmos é que ele é simples e a decomposição ótima encontrada por ele pode conter subconjuntos do quadrado elementar que não sejam convexos.

\subsection{Método Guloso}

Para alguns problemas de Otimização Combinatória é possível aplicar uma estratégia gulosa [15, p. 329][4, p. 157] para se obter uma solução ótima. O método guloso consiste em encontrar uma seqüência de soluções parciais (que são máximos ou mínimos locais do problema) que nos levam a uma solução ótima do problema (ou seja, a um máximo ou mínimo global do problema). Por exemplo, se o problema é maximizar uma dada função e é conhecido que uma solução parcial cujo valor digamos seja 25 , e este valor pode crescer para 30, 28 ou 45, então a solução de valor 45 é a próxima solução parcial.

Quando um problema de Otimização Combinatória pode ser resolvido por uma estratégia gulosa, o correspondente algoritmo é chamado de algoritmo guloso e, normalmente, eles são bastante simples. No entanto, na maioria dos casos, provar a corretude desses algoritmos pode ser uma tarefa não muito trivial.

\subsection{Decomposição Ótima de Elementos Estruturan- tes Convexos}

Esta seção apresenta o algoritmo guloso proposto para a decomposição de EE's convexos (veja a Subseção 5.3.2). Mas antes, introduziremos na Subseção 5.3.1, algumas proprie- 
dades necessárias para a apresentação do algoritmo. Para isto, faremos uso de algumas propriedades já vistas nos Capítulos 3 e 4 .

\subsubsection{Propriedades Necessárias para o Algoritmo}

A seguinte proposição [49] afirma que todo EE convexo tem uma decomposição seqüencial.

Proposição 5.1 Se A é um EE convexo, então A tem uma decomposição seqüencial.

Como uma conseqüência da Proposição 3.15, temos que a relação de invariança é transitiva.

Proposição 5.2 Sejam X, Y e Z EE's. Se $X$ é invariante de $Y$ e $Y$ é invariante de $Z$, então $X$ é um invariante de $Z$.

Prova: Uma vez que $X$ é invariante de $Y$ e $Y$ é invariante de $Z$, então $Y=(Y \ominus X) \oplus X$ e $Z=(Z \ominus Y) \oplus Y=(Z \ominus Y) \oplus((Y \ominus X) \oplus X)=((Z \ominus Y) \oplus(Y \ominus X)) \oplus X$. Assim, pela Proposição $3.15, X$ é um invariante de $Z$.

Como uma conseqüência das Proposições 3.14 e 4.16, temos o seguinte corolário.

Corolário 5.3 Sejam $A$ e $X$ dois EE's. Se existe um $i \in\{0,1, \cdots, 7\}$ tal que $\nu_{i}(A)<$ $\nu_{i}(X)$, então $X$ não é um invariante de $A$.

Prova: Suponha por absurdo que $X$ é um invariante de $A$. Assim, pela Proposição 3.14, existe um EE $Y$ tal que $A=Y \oplus X$. Dessa forma, pela Proposição 4.16, $\nu(A)=\nu(Y)+$ $\nu(X)$ e, portanto, para todo $i \in\{0,1, \cdots, 7\}, \nu_{i}(A) \geq \nu_{i}(X)$. Mas isto é uma contradição, pois por hipótese, existe $i \in\{0,1, \cdots, 7\}$ tal que $\nu_{i}(A)<\nu_{i}(X)$. Portanto, $X$ não é um invariante de $A$.

Note que o Corolário 5.3 fornece somente uma condição necessária para verificar se um EE $X$ é um invariante de $A$. Assim, o fato de que $\nu_{i}(A) \geq \nu_{i}(X)$, para todo $i \in$ $\{0,1, \cdots, 7\}$, não implica que $X$ é um invariante de $A$.

\subsubsection{Apresentação do Algoritmo Proposto}

O algoritmo proposto, chamado de DECCoN, está baseado em uma simples idéia que apresentamos a seguir. A seqüência SeqQ $=\left[B_{1}, B_{2}, \cdots, B_{n}\right]$ (veja sua definição na página 43), mencionada no Passo 01 do algoritmo, pode ser construída antes de rodar DECCoN. No início de cada iteração, temos uma solução parcial $\mathrm{S}_{j}(A)=\left[C_{1}, C_{2}, \cdots, C_{j}\right]$ e um conjunto 
$Y_{j}=A \ominus C_{1} \ominus C_{2} \ominus \cdots \ominus C_{j}$ (primeira iteração: Passos 02 e 03; próximas iterações: Passos 11 e 12). Se $\rho\left(Y_{j}\right)=(0,0)$, então o algoritmo pára e $\mathrm{S}_{j}(A)=\left[C_{1}, C_{2}, \cdots, C_{j}\right]$ é a decomposição ótima de $A$ (Passos 05 e 15). Caso contrário, um elemento $B_{i}$ de SeqQ é selecionado. Se $B_{i}$ não é um invariante de $Y_{j}$ (Passos 06 e 08), então o algoritmo seleciona o próximo elemento $B_{i+1}$ de SeqQ e uma nova iteração começa (Passos 07, 09 e 05). Caso contrário, $B_{i}$ é usado para se obter uma nova solução parcial $\mathrm{S}_{j+1}(A)=\mathrm{S}_{j}(A) \cdot\left[B_{i}\right]$ (Passo 11) e um novo conjunto $Y_{j+1}=Y_{j} \ominus B_{i}$ (Passo 12) e, em seguida, uma nova interação é iniciada (Passo 05).

Algoritmo DeCCon:

Entrada: Um EE convexo $A$ tal que $|A| \geq 2$.

Saída: Uma decomposição ótima de $A$.

01: Seja SeqQ $=\left[B_{1}, B_{2}, \cdots, B_{n}\right]$.

02: $\quad \mathrm{S}_{0}(A) \leftarrow[] / *$ seqüência vazia */

03: $\quad Y_{0} \leftarrow A$

$04: \quad i \leftarrow 1 ; j \leftarrow 0$

05: $\quad$ se $\rho\left(Y_{j}\right)=(0,0)$ então vá para o Passo 15

06: $\quad$ se existe $k \in\{0,1, \cdots, 7\}$ tal que $\nu_{k}\left(Y_{j}\right)<\nu_{k}\left(B_{i}\right)$ então

07: $\quad i \leftarrow i+1$ e vá para o Passo $05 / *$ pelo Corolário 5.3, $B_{i}$ não é um invariante de $Y_{j}^{*} /$

08: $\quad$ se $B_{i}$ não é um invariante de $Y_{j}$ então

09: $\quad i \leftarrow i+1$ e vá para o Passo 05

10: $\quad / * B_{i}$ é um invariante de $Y_{j}^{*} /$

11: $\mathrm{S}_{j+1}(A) \leftarrow \mathrm{S}_{j}(A) \cdot\left[B_{i}\right]$

12: $\quad Y_{j+1} \leftarrow Y_{j} \ominus B_{i}$

13: $j \leftarrow j+1$

14: vá para o Passo 05

15: Devolva a seqüência $\mathrm{S}_{j}(A)$.

Dependendo da ordem escolhida para construir a seqüência $\operatorname{SeqQ}=\left[B_{1}, B_{2}, \cdots, B_{n}\right]$, diferentes decomposições podem ser obtidas pelo algoritmo DeCCon. Nós ordenamos os elementos da sequiência SeqQ em ordem decrescente segundo a soma das coordenadas dos vetores retangulares de cada elemento em SeqQ, isto é, $\rho_{0}\left(B_{1}\right)+\rho_{1}\left(B_{1}\right) \geq \rho_{0}\left(B_{2}\right)+$ $\rho_{1}\left(B_{2}\right) \geq \cdots \geq \rho_{0}\left(B_{n}\right)+\rho_{1}\left(B_{n}\right)$, e, ao mesmo tempo, se $\rho_{0}\left(B_{i}\right)+\rho_{1}\left(B_{i}\right)=\rho_{0}\left(B_{j}\right)+\rho_{1}\left(B_{j}\right)$, e $\left|B_{i}\right| \leq\left|B_{j}\right|$, então $B_{i}$ aparece antes de $B_{j}$ em SeqQ. Por exemplo, na Figura 5.1.b apresentamos os invariantes do EE $A$ (apresentado na Figura 5.1.a) segundo a ordem 


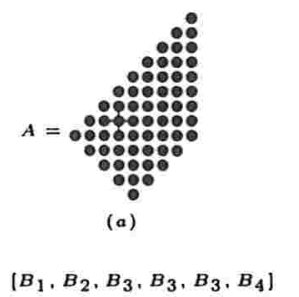

(c)

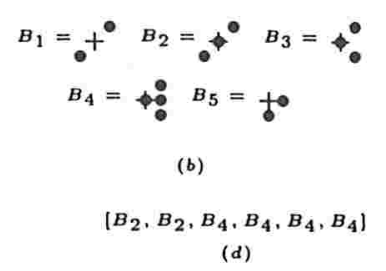

(c) Saída do algoritmo DecCon. (d) Saída do

Figura 5.1: $(a)$
algoritmo do Xu.

escolhida para construir SeqQ. Nesta figura, observe que $\rho_{0}\left(B_{1}\right)+\rho_{1}\left(B_{1}\right)=\rho_{0}\left(B_{2}\right)+$ $\rho_{1}\left(B_{2}\right)>\rho_{0}\left(B_{3}\right)+\rho_{1}\left(B_{3}\right)=\rho_{0}\left(B_{4}\right)+\rho_{1}\left(B_{4}\right)>\rho_{0}\left(B_{5}\right)+\rho_{1}\left(B_{5}\right),\left|B_{1}\right| \leq\left|B_{2}\right|$ e $\left|B_{3}\right| \leq\left|B_{4}\right|$.

Segundo esta ordenação, a cada iteração, o algoritmo DECCoN escolhe o EE em SeqQ cuja a soma de coordenadas do seu vetor retangular é a maior possível (e no caso dessa soma ser igual, então escolhe-se o EE de cardinalidade menor possível) para colocar na solução parcial. Esta escolha caracteriza a estratégia gulosa do algoritmo. Além disso, o algoritmo DECCON prefere escolher EE's não convexos em vez de EE's convexos para a decomposição de $A$, uma vez que EE's não convexos têm menos pontos do que os convexos. Assim, como a complexidade de tempo dos algoritmos que implementam erosões e dilatações dependem do número de pontos dos EE's que o caracterizam, então o algoritmo DECCON tem uma grande vantagem em relação ao algoritmo desenvolvido por $\mathrm{Xu}$, uma vez que, pelo algoritmo do $\mathrm{Xu}$, todos os elementos encontrados são subconjuntos convexos do quadrado elementar [49].

Por exemplo, nas Figuras 5.1.c e 5.1.d estão apresentados, respectivamente, as saídas do algoritmo proposto e do algoritmo do Xu. Neste particular exemplo, a diferença é somente de quatro pontos, mas para EE's maiores, esta diferença pode ser considerável (veja a Seção 5.4).

Na Figura 5.2, mostramos uma simples aplicação do algoritmo DECCoN para encontrar uma decomposição do EE convexo $A$ (apresentado na Figura 5.2.a). A Figura 5.2.b apresenta os invariantes de $A$ que são subconjuntos do quadrado elementar. $\mathrm{O}$ vetor projeção, o vetor retangular e o limite inferior de $A$ são mostrados na Figura 5.2.c. A Figura 5.2.d apresenta a seqüência vazia $S_{0}(A)$ e o conjunto $Y_{0}=A$ que são inicializados, respectivamente, nos Passos 02 e 03. O primeiro invariante de $Y_{0}$ em SeqQ (esta seqüência é previamente construída antes de rodar o algoritmo) é $B_{1}$. A Figura 5.2.e apresenta o conjunto $Y_{1}=Y_{0} \ominus B_{1}$ (construído no Passo 12), e a solução parcial $S_{1}(A)=\left[B_{1}\right]$ (construída no Passo 11). De uma maneira similar, a Figura 5.2.f apresenta o conjunto $Y_{2}=Y_{1} \ominus B_{2}$ (construído no Passo 12), onde $B_{2}$ é o próximo elemento da seqüência SeqQ que é invariante de $Y_{1}$, e a solução parcial $S_{2}(A)=\left[B_{1}, B_{2}\right]$ (construída no Passo 11). As Figuras 5.2.g, 5.2.h and 5.2.i apresentam os conjuntos $Y_{3}=Y_{2} \ominus B_{3}, Y_{4}=Y_{3} \ominus B_{3}$ e $Y_{5}=Y_{4} \ominus B_{3}$ (construídos no Passo 12), onde $B_{3}$ é o próximo elemento da seqüência SeqQ que é invariante de $Y_{3}, Y_{4}$ e $Y_{5}$; e as soluções parciais $S_{3}(A), S_{4}(A)$ e $S_{5}(A)$ (cons- 


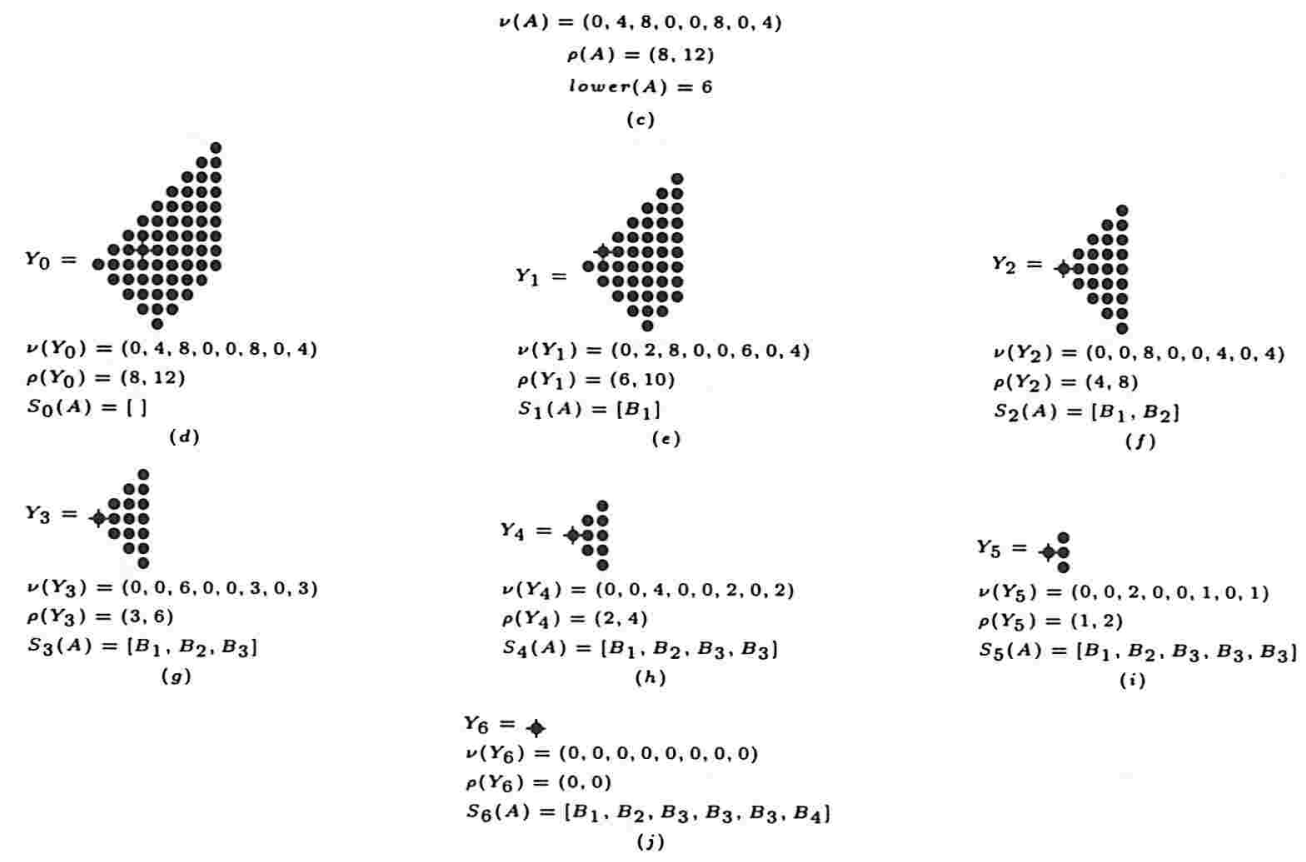

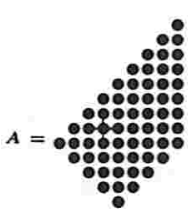

(a)

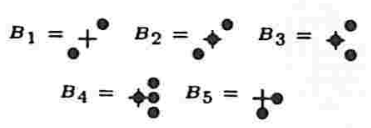

(b)

Figura 5.2: Aplicação do algoritmo DECCon para um exemplo simples.

truídas no Passo 11). Na Figura 5.2.j, a solução parcial $S_{6}(A)$ e o conjunto $Y_{6}=Y_{5} \ominus B_{4}$ são construídos nos Passos 11 e 12, respectivamente, uma vez que $B_{4}$ é o próximo elemento na seqüência SeqQ que é invariante de $Y_{5}$. Além disso, uma vez que $Y_{6}$ é um conjunto unitário, então $\rho\left(Y_{6}\right)=(0,0)$, e, conseqüentemente, o algoritmo pára. Como $A \equiv B_{1} \oplus B_{2} \oplus B_{3} \oplus B_{3} \oplus B_{3} \oplus B_{4}$, então $S_{6}(A)$ é uma decomposição ótima do EE convexo $A$, uma vez que lower $(A)=6$. Vale a pena observar aqui que a solução $S_{6}(A)$ é uma subseqüência de Seqlnv $[A]=\left[B_{1}, B_{2}, B_{2}, B_{3}, B_{3}, B_{3}, B_{4}, B_{4}, B_{4}, B_{4}, B_{5}, B_{5}\right]$.

\subsection{Resultados Experimentais}

Nesta seção, apresentaremos alguns resultados experimentais da aplicação do algoritmo do $\mathrm{Xu}$ e do algoritmo proposto e compararemos o desempenho desses algoritmos para encontrar decomposições ótimas de EE's convexos.

Estes experimentos foram executados usando uma máquina com processador Pentium III, $450 \mathrm{MHz}$. O tempo de processamento da CPU foi medido em segundos (s). 


\begin{tabular}{|c|c|c|c|c|}
\hline & \multicolumn{2}{|c|}{$\begin{array}{c}\text { Tempo Médio para } \\
\text { encontrar uma } \\
\text { solução ótima }\end{array}$} & \multicolumn{2}{c|}{$\begin{array}{c}\text { Número médio de } \\
\text { pontos na } \\
\text { solução ótima }\end{array}$} \\
\hline $\begin{array}{c}\text { Subconjuntos do } \\
\text { retângulo }\end{array}$ & $\begin{array}{c}\text { Algoritmo } \\
\text { do Xu }\end{array}$ & $\begin{array}{c}\text { Algoritmo } \\
\text { DecCoN }\end{array}$ & $\begin{array}{c}\text { Algoritmo } \\
\text { do Xu }\end{array}$ & $\begin{array}{c}\text { Algoritmo } \\
\text { DecCoN }\end{array}$ \\
\hline $20 \times 21$ & $0.22 \mathrm{~s}$ & $0.23 \mathrm{~s}$ & 58 & 32 \\
$38 \times 40$ & $0.23 \mathrm{~s}$ & $0.27 \mathrm{~s}$ & 114 & 57 \\
$56 \times 57$ & $0.23 \mathrm{~s}$ & $0.26 \mathrm{~s}$ & 176 & 84 \\
$73 \times 73$ & $0.26 \mathrm{~s}$ & $0.28 \mathrm{~s}$ & 234 & 110 \\
$89 \times 89$ & $0.28 \mathrm{~s}$ & $0.28 \mathrm{~s}$ & 297 & 137 \\
$109 \times 108$ & $0.25 \mathrm{~s}$ & $0.33 \mathrm{~s}$ & 358 & 164 \\
$124 \times 124$ & $0.32 \mathrm{~s}$ & $0.54 \mathrm{~s}$ & 420 & 191 \\
$141 \times 144$ & $0.29 \mathrm{~s}$ & $0.42 \mathrm{~s}$ & 476 & 216 \\
$160 \times 162$ & $0.31 \mathrm{~s}$ & $0.47 \mathrm{~s}$ & 539 & 244 \\
$176 \times 179$ & $0.35 \mathrm{~s}$ & $0.56 \mathrm{~s}$ & 611 & 275 \\
$192 \times 193$ & $0.37 \mathrm{~s}$ & $0.63 \mathrm{~s}$ & 661 & 297 \\
$214 \times 210$ & $0.41 \mathrm{~s}$ & $0.75 \mathrm{~s}$ & 729 & 326 \\
$229 \times 227$ & $0.45 \mathrm{~s}$ & $0.86 \mathrm{~s}$ & 786 & 352 \\
$246 \times 247$ & $0.48 \mathrm{~s}$ & $0.97 \mathrm{~s}$ & 844 & 377 \\
\hline
\end{tabular}

Tabela 5.1: Tempo médio para encontrar uma decomposição ótima e a média do número de pontos nesta decomposição.

Nós aplicamos o algoritmo do Xu e o algoritmo DECCon para encontrar uma decomposição ótima de aproximadamente $700 \mathrm{EE}$ 's convexos. Estes EE's foram gerados aleatoriamente.

Na Seção 5.6, provaremos que, dado um EE convexo $A$, a complexidade de tempo de DECCon e do algoritmo do Xu para encontrar uma decomposição ótima são, respectivamente, $O\left(n^{3}\right)$ e $O\left(n^{2}\right)$, onde $n$ é o valor máximo entre as duas coordenadas do vetor retangular de $A$, ou seja, $n=\max \left\{\rho_{0}(A), \rho_{1}(A)\right\}$. Assim, na Tabela 5.1, nas colunas $2-3$, podemos ver que o tempo médio para o algoritmo do Xu encontrar uma solução ótima é melhor do que o algoritmo DECCON.

Seja $A$ um EE. Seja $\mathrm{S}=\left[C_{1}, C_{2}, \cdots, C_{j}\right]$ uma decomposição de $A$. Denotaremos por $\sigma(\mathrm{S})$ a soma da cardinalidade de todos elementos na seqüência $\mathrm{S}$, isto é, $\sigma(\mathrm{S})=$ $\left|C_{1}\right|+\left|C_{2}\right|+\cdots+\left|C_{j}\right|$.

Dado um EE convexo $A$, denotamos $\operatorname{Decomp}(A)$ e $\mathrm{Xu}(A)$ a decomposição de $A$ encontrada, respectivamente, por DECCON e pelo algoritmo do Xu.

Seja $\mathcal{A}=\left\{A_{1}, A_{2}, \cdots, A_{m}\right\}$ uma coleção de EE's convexos. Definimos o número médio de pontos na solução ótima encontrada por DECCON e pelo algoritmo do Xu, respectivamente, como $\frac{\left(\sum_{i=1}^{m} \sigma\left(\operatorname{Decomp}\left(A_{i}\right)\right)\right)}{m} \mathrm{e} \frac{\left(\sum_{i=1}^{m} \sigma\left(\mathrm{Xu}\left(A_{i}\right)\right)\right)}{m}$.

O número médio de pontos na solução ótima encontrada por DECCoN é menor do que a obtida pelo algoritmo do Xu (colunas $4-5$ da Tabela 5.1). Conseqüentemente, a complexidade computacional para aplicar dilatações ou erosões usando as soluções dadas por DeCCon são melhores que as soluções encontradas pelo algoritmo do Xu.

Para finalizar esta seção, observamos que um próximo passo deste trabalho pode ser 
fazer uma versão simplificada do algoritmo DECCoN colocando-se na seqüência SeqQ somente os elementos de SeqQ que são subconjuntos convexos do quadrado elementar. Com isto, de acordo com uma propriedade de subconjuntos convexos [34, Proposição 3.3, p. 306], o teste do Passo 08 pode ser simplicado e executado em tempo $O(1)$. Além disso, não seria necessário construir os conjuntos $Y_{j}$ (ou seja, o Passo 12 seria desconsiderado). Dessa forma, com esta simplificação, o algoritmo teria mesma complexidade do algoritmo do Xu.

\subsection{Corretude do Algoritmo}

Nesta seção, provaremos a corretude do algoritmo. Embora o algoritmo DECCoN seja simples, sua prova de corretude não é intuitiva. Dividiremos esta seção em três partes. Na primeira, apresentaremos algumas propriedades do fecho convexo necessárias para a prova da corretude do algoritmo. Na segunda parte, provaremos que, dado um EE convexo $A$, a saída do algoritmo é uma decomposição de $A$. Finalmente, na última parte, mostraremos que esta decomposição encontrada é otima.

\subsubsection{Propriedades do Fecho Convexo para Elementos Estrutu- rantes Convexos}

Esta subseção fornece algumas propriedades do fecho convexo que serão utilizadas para a prova de corretudo do algoritmo.

Lema 5.4 Seja A um EE convexo. Se $X$ e $Y$ são EE's tais que $X \oplus Y=A$, então $C(X) \oplus Y=A$.

Prova: Por um lado, $A \subseteq C(X) \oplus Y$, uma vez que, pelo Lema 4.4.a, $X \subseteq C(X)$ e, pela Proposição 3.8.g, $X \oplus Y \subseteq C(X) \oplus Y$. Por outro lado, $C(X) \oplus Y \subseteq A$, uma vez que, pelo Lema 4.7, $C(X) \oplus Y \subseteq C(X \oplus Y)=C(A)=A$. Portanto, $C(X) \oplus Y=A$. e 3.15 .

O próximo resultado é uma conseqüência imediata do Lema 5.4 e das Proposições 3.14

Lema 5.5 Seja A um EE convexo. Se $X$ é um invariante de $A$, então $C(X)$ também é um invariante de $A$.

Prova: Como $X$ é um invariante de $A$, então, pela Proposição 3.14, existe um EE $Y \in \mathbb{Z}^{2}$ tal que $X \oplus Y=A$. Pelo Lema 5.4, $C(X) \oplus Y=A$. E, pela Proposição 3.15, $C(X)$ é um invariante de $A$. 
Lema 5.6 Seja $A$ um EE convexo. Se $X$ é um invariante de $A$, então $A \ominus X$ é um EE convexo.

Prova: Dividimos a prova deste lema em duas partes. Por um lado, pelo Lema 4.4.a, $A \ominus X \subseteq C(A \ominus X)$. Por outro lado, $C(A \ominus X) \subseteq A \ominus X$. De fato, uma vez que $X$ é um invariante de $A,(A \ominus X) \oplus X=A$ e, pelo Lema $5.4, C(A \ominus X) \oplus X=A$. Assim, pela relação de adjunção, dada pela Proposição 3.11, $C(A \ominus X) \subseteq A \ominus X$.

Logo, $C(A \ominus X)=A \ominus X$ e, portanto, $A \ominus X$ é um EE convexo.

\subsubsection{A Saída do Algoritmo é uma Decomposição}

Nesta subseção, provaremos que, dado um EE convexo $A$, a saída do algoritmo DECCon é uma decomposição de $A$. Para isso, precisamos estudar algumas propriedades do algoritmo proposto.

A cada iteração, um EE $Y_{j}$ é construído pelo algoritmo. Quando $Y_{j}$ é o EE considerado em uma iteração do algoritmo, $\mathrm{S}_{j}(A)=$ [] (seqüência vazia), se $j=0$; ou $\mathrm{S}_{j}(A)=$ $\left[C_{1}, C_{2}, \cdots, C_{j}\right]$, se $j>0$, é a solução parcial atual de $A$, ou simplesmente, solução parcial de $A$.

Para provar que a saída do algoritmo $\mathrm{S}_{j}(A)=\left[C_{1}, C_{2}, \cdots, C_{j}\right]$ é uma decomposição de um dado EE $A$, devemos mostrar que $C_{1} \oplus C_{2} \oplus \cdots \oplus C_{j} \equiv A$. Dados dois EE's $A$ e $Y$, a proposição seguinte nos fornece uma definição equivalente para $A \equiv Y$.

Proposição 5.7 Sejam $A$ e $Y$ dois EE's. Então $Y \equiv A$ se, e somente se, $Y$ é invariante de $A$ e $\rho(Y)=\rho(A)$.

Prova: $(\Rightarrow)$ Se $Y \equiv A$, então, existe $h \in \mathbb{Z}^{2}$ tal que $A=Y_{h}=Y \oplus\{h\}$. Logo, pela Proposição 3.15, $Y$ é invariante de $A$. Uma vez que $\rho\left(Y_{h}\right)=\rho(Y)$, então, $\rho(Y)=\rho(A)$.

$(\Leftarrow)$ Uma vez que $Y$ é invariante de $A$, pela Proposição 3.14, existe um EE $X$ tal que $A=X \oplus Y$. Pela Proposição 4.17, $\rho(A)=\rho(X)+\rho(Y)$. Uma vez que $\rho(A)=\rho(Y)$, então $\rho(X)=(0,0)$ e, portanto, $|X|=1$. Seja $h \in \mathbb{Z}^{2}$ tal que $X=\{h\}$. Neste caso, a adição de Minkowski $X \oplus Y=A$ é uma translação do conjunto $Y$ por $h$. Logo, $Y \equiv A$.

Agora, vamos introduzir duas definições. Seja SeqQ $=\left[B_{1}, B_{2}, \cdots, B_{n}\right]$ e seja $A$ um EE convexo. Quando $Y_{j}$ é o EE considerado pelo algoritmo, ou seja, $Y_{j}$ é o EE considerado em uma dada iteração do algoritmo, chamamos de seqüência decrescente atual de $A$, ou simplesmente, seqüência decrescente de $A$, a seqüência formada por todos EE's $Y_{i}$ gerados pelo algoritmo (nos Passos 03 e 12) até $Y_{j}$ (inclusive), ou seja, a seqüência $\left[Y_{0}, Y_{1}, \cdots, Y_{j}\right]$. Se $S_{j}(A)=\left[C_{1}, C_{2}, \cdots, C_{j}\right]$ é a solução parcial, chamamos de seqüência restante de $Y_{j}$ a seqüência Restante $\left[Y_{j}\right]=\operatorname{SeqQ}$, se $j=0$; ou Restante $\left[Y_{j}\right]=\left[C_{j}=B_{i}, B_{i+1}, \cdots, B_{n}\right]$, se 
$j>0$, onde $B_{i}, B_{i+1}, \cdots, B_{n} \in$ SeqQ. Observe que, pela definição de Restante $\left[Y_{j}\right], B_{i}=C_{j}$ é o elemento de SeqQ usado para construir $Y_{j}$ (no Passo 12).

Assim, pelas definições dadas acima, dado um $\mathrm{EE}$ convexo $A$, podemos ver claramente que, se $\left[Y_{0}, Y_{1}, \cdots, Y_{j}\right]$ e $\mathrm{S}_{j}(A)=\left[C_{1}, C_{2}, \cdots, C_{j}\right]$ são, respectivamente, a seqüência decrescente atual de $A$ e a solução parcial atual, então pela construção do algoritmo (Passos 10 e 11), para cada $i \in\{1,2, \cdots, j\}, C_{i}$ é um invariante de $Y_{i-1}$, e, além disso, $C_{i}$ é utilizado para construir $Y_{i}$, isto é, $Y_{i}=Y_{i-1} \ominus C_{i}$. Se $\rho\left(Y_{j}\right) \neq(0,0)$, então o próximo elemento $C_{j+1}$ para gerar a nova solução parcial $\mathrm{S}_{j+1}(A) \leftarrow \mathrm{S}_{j}(A) \cdot\left[C_{j+1}\right]$ (no Passo 11) é um elemento de Restante $\left[Y_{j}\right]$. Seja $B_{r}$ o elemento de Restante $\left[Y_{j}\right]$ tal que $B_{r}=C_{j+1}$. Pela construção do algoritmo, $B_{r}$ é o primeiro elemento de Restante $\left[Y_{j}\right]$ tal que $B_{r}$ é um invariante de $Y_{j}$, ou seja, Restante $\left[Y_{j}\right]=\left[C_{j}=B_{i}, B_{i+1}, \cdots, B_{r-1}, C_{j+1}=B_{r}, B_{r+1} \cdots, B_{n}\right]$ e todos elementos na seqüência $\left[B_{i}, B_{i+1}, \cdots, B_{r-1}\right]$ não são invariantes de $Y_{j}$.

Dado um EE convexo $A$, para provar a corretude do algoritmo proposto, temos que mostrar que as seguintes duas afirmações são válidas.

(1) Se $\rho\left(Y_{j}\right)=(0,0)$, então $A \equiv C_{1} \oplus C_{2} \oplus \cdots \oplus C_{j}$, onde $\left[C_{1}, C_{2}, \cdots, C_{j}\right]$ é a saída do algoritmo;

(2) Existe um elemento $B$ em Restante $\left[Y_{j}\right]$ tal que $B$ é um invariante de $Y_{j}$ se, e somente se, $\rho\left(Y_{j}\right) \neq(0,0)$.

Note que a Afirmação (1) guarante que quando $\rho\left(Y_{j}\right)=(0,0)$, a saída do algoritmo é uma decomposição, enquanto a Afirmação (2) guarante que, se existe um elemento $B$ em Restante $\left[Y_{j}\right]$ tal que $B$ é um invariante de $Y_{j}$, então $\rho\left(Y_{j}\right) \neq(0,0)$. Assim, neste caso, a condição no Passo 05 não é satisfeita e, conseqüentemente, a próxima iteração do algoritmo é sempre possível. Além disso, a Afirmação (2) também guarante que se todos elementos em Restante $\left[Y_{j}\right]$ não são invariantes de $Y_{j}$, então $\rho\left(Y_{j}\right)=(0,0)$ e, portanto, o algoritmo pára.

Para provar as Afirmações (1) e (2), vamos introduzir mais uma definição. Dado um EE convexo $A$. Se $\mathrm{S}_{j}(A)=\left[C_{1}, C_{2}, \cdots, C_{j}\right]$ é a solução parcial atual, chamamos de seqüência crescente atual de $A$, ou simplesmente, seqüência crescente de $A$, a seqüência $\left[X_{0}, X_{1}, \cdots, X_{j}\right]$, onde $X_{0}=\{o\}$ (isto é, o conjunto unitário que contém a origem) e $X_{i}=X_{i-1} \oplus C_{i}$, para $1 \leq i \leq j$. Assim, para provar a Afirmação (1), temos que mostrar que, se $\rho\left(Y_{j}\right)=(0,0)$, então $X_{j} \equiv A$, uma vez que $X_{j}=C_{1} \oplus C_{2} \oplus \cdots \oplus C_{j}$.

\subsubsection{Prova da Afirmação (1)}

Seja $A$ um EE convexo. Sejam $\left[Y_{0}, Y_{1}, \cdots, Y_{j}\right]$ e $\left[X_{0}, X_{1}, \cdots, X_{j}\right]$, respectivamente, as seqüências decrescente e crescente de $A$. Os seguintes resultados (Lemas 5.8, 5.9 e 5.10) fornecem algumas propriedades para provar a Afirmação (1). O Lema 5.8 mostra que $A=Y_{j} \oplus X_{j}$. O Lema 5.9, que é uma consequiência da Proposição 3.15 e do Lema 5.8, 
mostra que $X_{j}$ é um invariante de $A$. O Lema 5.10 motra que se $\rho\left(Y_{j}\right)=(0,0)$, então $\rho\left(X_{j}\right)=\rho(A)$. Finalmente, usando os resultados dos Lemas 5.9 e 5.10 , a Proposição 5.11 prova a Afirmação (1) mostrando que se $\rho\left(Y_{j}\right)=(0,0)$, então $X_{j} \equiv A$.

Lema 5.8 Seja $A$ um EE convexo. Se $\left[Y_{0}, Y_{1}, \cdots, Y_{j}\right]$ e $\left[X_{0}, X_{1}, \cdots, X_{j}\right]$ são, respectivamente, as seqüências decrescente e crescente atuais de $A$, então $A=Y_{j} \oplus X_{j}$ e $Y_{j}=A \ominus X_{j}$.

Prova: Provaremos este lema por indução em $j$. Quando $j=0$, é óbvio que $A=Y_{0} \oplus X_{0}$ e $Y_{0}=A \ominus X_{0}$, uma vez que $Y_{0}=A$ e $X_{0}=\{o\}$. Se $j>0$, suponha, por hipótese de indução, que este lema é verdadeiro para $j-1$, ou seja, $A=Y_{j-1} \oplus X_{j-1}$ e $Y_{j-1}=A \ominus X_{j-1}$. Provaremos que $A=Y_{j} \oplus X_{j}$ e $Y_{j}=A \ominus X_{j}$.

Se $\mathrm{S}_{j}(A)=\left[C_{1}, C_{2}, \cdots, C_{j}\right]$ é a solução parcial atual, então $C_{j}$ é um invariante de $Y_{j-1}$, $Y_{j}=Y_{j-1} \ominus C_{j}$ e $X_{j}=X_{j-1} \oplus C_{j}$.

Assim, a prova para a primeira igualdade é dada da seguinte forma.

$$
\begin{aligned}
A & =Y_{j-1} \oplus X_{j-1} & & \text { (por hipótese de indução) } \\
& =\left(\left(Y_{j-1} \ominus C_{j}\right) \oplus C_{j}\right) \oplus X_{j-1} & & \text { (uma vez que } \left.C_{j} \text { é invariante de } Y_{j-1}\right) \\
& =\left(Y_{j-1} \ominus C_{j}\right) \oplus\left(X_{j-1} \oplus C_{j}\right) & & \text { (pela comutatividade e associatividade } \\
& =Y_{j} \oplus X_{j} . & & \text { da adição de Minkowski) }
\end{aligned}
$$

E, para a segunda igualdade,

$$
\begin{aligned}
Y_{j} & =Y_{j-1} \ominus C_{j} & & \\
& =\left(A \ominus X_{j-1}\right) \ominus C_{j} & & \text { (por hipótese de indução) } \\
& =A \ominus\left(X_{j-1} \oplus C_{j}\right) & & \text { (pela Proposição 3.9.f) } \\
& =A \ominus X_{j} . & &
\end{aligned}
$$

O próximo resultado é uma conseqüência da Proposição 3.15 e do Lema 5.8 .

Lema 5.9 Seja $A$ um EE convexo. Se $\left[Y_{0}, Y_{1}, \cdots, Y_{j}\right]$ e $\left[X_{0}, X_{1}, \cdots, X_{j}\right]$ são, respectivamente, as seqüências decrescente e crescente atuais de $A$, então $Y_{j}$ e $X_{j}$ são invariantes de $A$.

Como conseqüência do Lema 5.9, o último $\operatorname{EE} X_{j}$ em qualquer seqüência crescente de $A$ é um invariante de $A$. Assim, em particular, quando $\rho\left(Y_{j}\right)=(0,0), X_{j}$ é um invariante de $A$. Um passo intermediário para provar a Afirmação (1) é mostrar que, se $\rho\left(Y_{j}\right)=(0,0)$, então $\rho\left(X_{j}\right)=\rho(A)$. 
Lema 5.10 Seja $A$ um EE convexo. Sejam $\left[Y_{0}, Y_{1}, \cdots, Y_{j}\right]$ e $\left[X_{0}, X_{1}, \cdots, X_{j}\right]$, respectivamente, as seqüências decrescente e crescente atuais de $A$. Se $\rho\left(Y_{j}\right)=(0,0)$, então $\rho\left(X_{j}\right)=\rho(A)$.

Prova: Pelo Lema 5.8, $A=Y_{j} \oplus X_{j}$. Assim, pela Proposição 4.17, $\rho(A)=\rho\left(Y_{j}\right)+\rho\left(X_{j}\right)$. Logo, $\rho\left(X_{j}\right)=\rho(A)$, uma vez que $\rho\left(Y_{j}\right)=(0,0)$.

Finalmente, a Proposição 5.11 prova a Afirmação (1).

Proposição 5.11 Seja $A$ um EE convexo. Sejam $\left[Y_{0}, Y_{1}, \cdots, Y_{j}\right]$ e $\left[X_{0}, X_{1}, \cdots, X_{j}\right]$ as seqüencias decrescente e crescente atuais de $A$. Se $\rho\left(Y_{j}\right)=(0,0)$, então $X_{j} \equiv A$.

Prova: Pelo Lema 5.9, $X_{j}$ é um invariante de $A$. Uma vez que $\rho\left(Y_{j}\right)=(0,0)$, então, pelo Lema 5.10, $\rho\left(X_{j}\right)=\rho(A)$. Assim, pela Proposição 5.7, $X_{j} \equiv A$.

\subsubsection{Prova da Afirmação (2)}

Dizemos que as seqüências decrescente e crescente de $A,\left[Y_{0}, Y_{1}, \cdots, Y_{j}\right]$ e $\left[X_{0}, X_{1} \cdots, X_{j}\right]$, são maximais se, e somente se, não existe um elemento $B$ em Restante $\left[Y_{j}\right]$ tal que $B$ é um invariante de $Y_{j}$. Obviamente, a seqüência decrescente atual $\left[Y_{0}, Y_{1}, \cdots, Y_{j}\right]$ é maximal se, e somente se, a seqüência crescente atual $\left[X_{0}, X_{1}, \cdots, X_{j}\right]$ é maximal.

Dado um EE convexo $A$, para mostrar a corretude do algoritmo, falta provarmos a Afirmação (2), ou seja, temos que mostrar que $\rho\left(Y_{j}\right) \neq(0,0)$ se, e somente se, a seqüência decrescente (equivalentemente, crescente) atual de $A$ não é maximal. Mas antes disso, precisamos do seguinte resultado.

Lema 5.12 Seja A um EE convexo. Seja $\left[Y_{0}, Y_{1}, \cdots, Y_{j}\right]$ a seqüência decrescente atual de $A$. Seja $B$ um elemento da seqüência $\mathrm{SeqQ}=\left[B_{1}, B_{2}, \cdots, B_{n}\right]$. Se $B$ é um invariante de $Y_{j}$, então $B$ é um elemento de Restante $\left[Y_{j}\right]$.

Prova: Provaremos este lema por indução em $j$. Se $j=0$, uma vez que Restante[ $\left[Y_{0}\right]=$ SeqQ e $B \in \operatorname{SeqQ}$, então $B$ é um elemento de Restante[ $\left.Y_{0}\right]$. Caso $j>0$, suponha, por hipótese de indução, que este lema vale para $j-1$, isto é, $B$ é um elemento de Restante $\left[Y_{j-1}\right]$. Provaremos que $B \in \operatorname{Restante}\left[Y_{j}\right]$.

Se Restante $\left[Y_{j}\right]=\left[B_{i}, B_{i+1}, \cdots, B_{n}\right]$, então $B_{i}$ é o EE de SeqQ usado pelo algoritmo para construir $Y_{j}$, ou seja, $Y_{j}=Y_{j-1} \ominus B_{i}$. Assim, $C_{j}=B_{i}$. Similarmente, se Restante $\left[Y_{j-1}\right]=$ $\left[B_{k}, B_{k+1}, \cdots, B_{n}\right]$, então $B_{k} \in$ SeqQ e $C_{j-1}=B_{k}$. Pela construção do algoritmo, $C_{j}$ é o primeiro elemento de Restante $\left[Y_{j-1}\right]$ tal que $C_{j}$ é um invariante de $Y_{j}$, ou seja, Restante $\left[Y_{j-1}\right]=\left[B_{k}, B_{k+1}, \cdots, B_{i-1}, B_{i}, B_{i+1}, \cdots, B_{n}\right]=\left[B_{k}, B_{k+1}, \cdots, B_{i-1}\right] \cdot$ Restante $\left[Y_{j}\right]$ e nenhum elemento na seqüência $\left[B_{k}, B_{k+1}, \cdots, B_{i-1}\right]$ é um invariante de $Y_{j}$. 
Uma vez que $B$ é um invariante de $Y_{j}$ e, por hipótese de indução, $B \in \operatorname{Restante}\left[Y_{j-1}\right]$, então, claramente, $B$ é um elemento de Restante $\left[Y_{j}\right]$.

Finalmente, o próximo resultado prova a Afirmação (2).

Proposição 5.13 Seja $A$ um EE convexo. A seqüência decrescente atual de $A$, digamos $\left[Y_{0}, Y_{1}, \cdots, Y_{j}\right]$, é maximal se, e somente se, $\rho\left(Y_{j}\right)=(0,0)$.

Prova: $\quad(\Leftarrow)$ Suponha por absurdo que a seqüência decrescente atual de $A$ não seja maximal. Seja $B$ um elemento de Restante $\left[Y_{j}\right]$ tal que $B$ é um invariante de $Y_{j}$.

Uma vez que Restante $\left[Y_{j}\right]$ é uma subseqüência de SeqQ, então $B$ tem pelo menos dois pontos e, conseqüentemente, $\rho_{0}(B)>0$ ou $\rho_{1}(B)>0$.

Uma vez que $B$ é um invariante de $Y_{j}$, então, pela Proposição 3.14 , existe um EE $X$ tal que $Y_{j}=X \oplus B$. Assim, pela Proposição 4.17, $\rho\left(Y_{j}\right)=\rho(X)+\rho(B)$. Logo, $\rho_{0}\left(Y_{j}\right) \geq \rho_{0}(B)>0$ ou $\rho_{1}\left(Y_{j}\right) \geq \rho_{1}(B)>0$, e, conseqüentemente, $\rho\left(Y_{j}\right) \neq(0,0)$. Mas isto contradiz a hipótese de que $\rho\left(Y_{j}\right)=(0,0)$. Portanto, a seqüência decrescente atual de $A$ é maximal.

$(\Rightarrow)$ Suponha por absurdo que $\rho\left(Y_{j}\right) \neq(0,0)$. Seja $\left[X_{0}, X_{1}, \cdots, X_{j}\right]$ a seqüência crescente atual de $A$. Assim, pelo Lema 5.8, $Y_{j}=A \ominus X_{j}$ e, pelo Lema 5.9, $X_{j}$ é um invariante de $A$.

Como $A$ é um EE convexo e $X_{j}$ é um invariante de $A$, então, pelo Lema 5.6, $Y_{j}=A \ominus X_{j}$ é também um EE convexo. Assim, pela Proposição 5.1, $Y_{j}$ tem uma decomposição seqüencial, digamos $\left[Z_{1}, Z_{2}, \cdots, Z_{\ell}\right]$. Uma vez que $\rho\left(Y_{j}\right) \neq(0,0)$, então $\left|Y_{j}\right| \geq 2$, e, conseqüentemente, um elemento em $\left[Z_{1}, Z_{2}, \cdots, Z_{\ell}\right]$, digamos $Z_{1}$, contém pelo menos dois pontos. Claramente, $Z_{1}$ é um invariante de $Y_{j}$. Uma vez que, pelo Lema $5.9, Y_{j}$ é um invariante de $A$, então, pela transitividade da relação de invariança (dada pela Proposição 5.2), $Z_{1}$ é um invariante de $A$.

Uma vez que $Z_{1}$ é um subconjunto do quadrado elementar e $\left|Z_{1}\right| \geq 2$, então existe um elemento $B$ em SeqQ $=\left[B_{1}, B_{2}, \cdots, B_{n}\right]$ tal que $B \equiv Z_{1}$. Uma vez que $Z_{1}$ é um invariante de $Y_{j}$, claramente, $B$ também é um invariante de $Y_{j}$, e, pelo Lema 5.12, $B$ é um elemento de Restante $\left[Y_{j}\right]$. Mas isto contradiz a hipótese de que a seqüência decrescente atual de $A$ é maximal. Portanto, $\rho\left(Y_{j}\right)=(0,0)$.

Para a completude desta seção, o próximo resultado mostra que a saída do algoritmo DecCon é uma decomposição de $A$.

Teorema 5.14 Seja $A$ um EE convexo. Se a seqüência crescente atual de $A$, digamos $\left[X_{0}, X_{1}, \cdots, X_{j}\right]$, é maximal, então $X_{j} \equiv A$.

Prova: Uma vez que a seqüência $\left[X_{0}, X_{1}, \cdots, X_{j}\right]$ é maximal, então a seqüência decrescente atual de $A$, digamos $\left[Y_{0}, Y_{1}, \cdots, Y_{j}\right]$, também é maximal. Assim, pela Propo- 
sição $5.13, \rho\left(Y_{j}\right)=(0,0)$. Logo, pelo Lema 5.10, $\rho\left(X_{j}\right)=\rho(A)$. Uma vez que, pelo Lema $5.9, X_{j}$ é um invariante de $A$, então, pela Proposição $5.7, X_{j} \equiv A$.

\subsubsection{A Saída do Algoritmo é uma Decomposição Ótima}

Na última subseção, o Teorema 5.14 mostra que se a seqüência crescente atual $\left[X_{0}, X_{1}, \cdots\right.$, $X_{j}$ ] de um dado EE convexo $A$ é maximal, então $X_{j} \equiv A$ e, portanto, a solução parcial atual $\mathrm{S}_{j}(A)=\left[C_{1}, C_{2}, \cdots, C_{j}\right]$, é uma decomposição de $A$. No final do Capítulo 4 , na Subseção 4.3.4, o Teorema 4.18 mostra um limite inferior para o comprimento das decomposições de $A$. Se provarmos que este limite inferior para decomposição de $A \equiv X_{j}$ é $j$ elementos (isto é, lower $\left(X_{j}\right)=j$ ), então $\mathrm{S}_{j}(A)$ será uma decomposição ótima de $A$, uma vez que $\mathrm{S}_{j}(A)$ contém exatamente $j$ elementos.

Assim, para provar que a saída do algoritmo DECCon é uma decomposição ótima $A$, vamos mostrar que, para cada seqüência crescente atual $\left[X_{0}, X_{1}, \cdots, X_{j}\right]$ de um dado EE convexo $A$, a correspondente solução parcial atual $\mathrm{S}_{j}(A)=\left[C_{1}, C_{2}, \cdots, C_{j}\right]$ é uma decomposição ótima de $X_{j}$, ou seja, $\operatorname{lower}\left(X_{j}\right)=j$. Para isso, precisamos de algumas definições e resultados preliminares.

Seja $A$ um EE. Seja R $=\left[C_{1}, C_{2}, \cdots, C_{j}\right]$ uma subseqüência qualquer de Seqlnv $[A]$. A seqüência $\mathrm{E}=\left[X_{0}, X_{1}, \cdots, X_{j}\right]$, onde $X_{0}=\{o\}$ e $X_{i}=X_{i-1} \oplus C_{i}$, para $i \in\{1,2, \cdots, j\}$, é chamada de seqüência gerada a partir de $\mathrm{R}$ e a seqüência $\mathrm{R}$ é chamada seqüência geradora de $\mathrm{E}$. Note que, por essa definição, a solução parcial atual $\mathrm{S}_{j}[A]$ é uma seqüência geradora da seqüência crescente atual de $A$.

Seja $A$ um EE. Seja R $=\left[C_{1}, C_{2}, \cdots, C_{j}\right]$ uma subseqüência de Seqlnv $[A]$. Denotaremos por $c_{a, b}(\mathrm{R})$, para $a, b \in\{0,1,2\}$, o número de EE's em $\mathrm{R}$ cujo vetor retangular é igual a $(a, b)$. Por exemplo, na Figura 5.2.i, claramente $\mathrm{S}_{5}(A)$ é uma subseqüência de $\operatorname{Seq} \operatorname{lnv}[A]$ e $c_{2,2}\left(\mathrm{~S}_{5}(A)\right)=2, c_{1,2}\left(\mathrm{~S}_{5}(A)\right)=3$ e $c_{a, b}\left(\mathrm{~S}_{5}(A)\right)=0$, para os outros valores de $a, b$. Claramente, $c_{0,0}(\mathrm{R})=0$, uma vez que qualquer elemento $B \in$ SeqQ contém pelo menos dois pontos, ou seja, $\rho(B) \neq(0,0)$.

Dado um EE $A$ e seja $\mathrm{R}=\left[C_{1}, C_{2}, \cdots, C_{j}\right]$ uma uma subseqüência de Seqlnv $[A]$. Dizemos que $\mathrm{R}$ é uma seqüência retangular de $A$ se, e somente se, todas as restrições abaixo são satisfeitas:
(a) $c_{2,0}(\mathrm{R}) \cdot c_{0,2}(\mathrm{R})=0$;
(b) $c_{2,0}(\mathrm{R}) \cdot c_{0,1}(\mathrm{R})=0$;
(c) $c_{0,2}(\mathrm{R}) \cdot c_{1,0}(\mathrm{R})=0$;
(d) $c_{2,1}(\mathrm{R}) \cdot c_{0,1}(\mathrm{R})=0$;
(e) $c_{1,2}(\mathrm{R}) \cdot c_{1,0}(\mathrm{R})=0$;
(f) $c_{1,1}(\mathrm{R})+c_{1,0}(\mathrm{R})+c_{0,1}(\mathrm{R}) \leq 1$
(g) $c_{2,0}(\mathrm{R}) \cdot c_{1,1}(\mathrm{R})=0$;
(h) $c_{2,0}(\mathrm{R}) \cdot c_{1,2}(\mathrm{R})=0$;
(i) $c_{0,2}(\mathrm{R}) \cdot c_{1,1}(\mathrm{R})=0$;
(j) $c_{0,2}(\mathrm{R}) \cdot c_{2,1}(\mathrm{R})=0$;
(k) $c_{2,1}(\mathrm{R}) \cdot c_{1,2}(\mathrm{R})=0$.

Seja $A$ um EE. Seja $\mathrm{R}=\left[C_{1}, C_{2}, \cdots, C_{j}\right]$ uma subseqüência de Seqlnv $[A]$. Definimos a seqüência $\mathrm{E}=\left[X_{0}, X_{1}, \cdots, X_{j}\right]$, onde $X_{i}=X_{i-1} \oplus C_{i}$, como a seqüência elemento 
retangular de $A$ se, e somente se, R é uma seqüência retangular de $A$. Além disso, note que $R$ é uma seqüência geradora de $E$.

O próximo resultado, provado na Seção 5.8, mostra que uma seqüência crescente atual construído pelo algoritmo DECCON de um dado EE convexo $A$ é uma seqüência elemento retangular de $A$.

Proposição 5.15 Se $\left[X_{0}, X_{1}, \cdots, X_{j}\right]$ uma seqüência crescente atual construído pelo algoritmo DecCon de um dado EE convexo $A$, então $\left[X_{0}, X_{1}, \cdots, X_{j}\right]$ é uma seqüência elemento retangular de $A$.

A próxima proposição mostra que se $\left[X_{0}, X_{1}, \cdots, X_{j}\right]$ é uma seqüência elemento retangular de um dado EE $A$, então $\operatorname{lower}\left(X_{j}\right)=j$. A prova desta proposição é dada na Seção 5.8 .

Proposição 5.16 Seja A um EE. Se $\mathrm{E}=\left[X_{0}, X_{2}, \cdots, X_{j}\right]$ é a seqüência elemento retangular de $A$, então lower $\left(X_{j}\right)=j$.

O próximo teorema fornece a prova de que a saída do algoritmo DECCoN é uma decomposição ótima de um dado EE convexo $A$.

Teorema 5.17 Seja $A$ um EE convexo. Seja $\left[X_{0}, X_{2}, \cdots, X_{j}\right]$ a seqüência crescente atual de A. Se $\left[X_{0}, X_{2}, \cdots, X_{j}\right]$ é maximal, então, $\mathrm{S}_{j}(A)=\left[C_{1}, C_{2}, \cdots, C_{j}\right]$ é uma decomposição ótima de $A$.

Prova: Pelo Teorema 5.14, $X_{j} \equiv A$. Assim, $\rho\left(X_{j}\right)=\rho(A)$, e conseqüentemente, lower $\left(X_{j}\right)=\operatorname{lower}(A)$. Como, pela Proposição $5.15,\left[X_{0}, X_{2}, \cdots, X_{j}\right]$ é uma seqüência elemento retangular de $A$, então, pela Proposição $5.16, \operatorname{lower}\left(X_{j}\right)=j$. Uma vez que $\mathrm{S}_{j}(A)$ contém exatamente $j$ elementos e lower $(A)=j$, então, claramente, $\mathrm{S}_{j}(A)$ é uma decomposição ótima de $A$.

Dado um EE convexo $A$, quando uma seqüência crescente atual maximal $\left[X_{0}, X_{1} \cdots\right.$, $X_{j}$ ] é encontrada pelo algoritmo, então, pelo Teorema $5.14, X_{j} \equiv A$ e, portanto, $\mathrm{S}_{j}(A)=$ $\left[C_{1}, C_{2}, \cdots, C_{j}\right]$ é uma decomposição de $A$. Pelo Teorema $5.17, \mathrm{~S}_{j}(A)$ é uma decomposição ótima de $A$, uma vez que $\operatorname{lower}(A)=\operatorname{lower}\left(X_{j}\right)=j$.

\subsection{Complexidade do Algoritmo}

Nesta seção, discutiremos a complexidade de tempo do algoritmo DecCon. Para isso, dado um EE convexo, usaremos, nesta seção, a seguinte notação: 
- $m_{p}$ denota o número de pontos de $A$;

- $m_{s}$ denota o número total de iterações que foram efetuadas pelo algoritmo;

- $n$ denota o valor máximo entre as duas coordenadas do vetor retangular de $A$, ou seja, $n=\max \left\{\rho_{0}(A), \rho_{1}(A)\right\}$.

Claramente, o número de pontos de um dado EE convexo $A$ é $m_{p}=O\left(n^{2}\right)$. A complexidade de tempo do algoritmo do Xu [49] para decompor EE's convexos é $O\left(n^{2}\right)$. Na proposição seguinte, mostramos que $m_{s}=O(n)$.

Proposição 5.18 Se A é um EE convexo, então o número total de iterações do algoritmo DECCON é $m_{s}=O(n)$.

Prova: $\quad$ Para cada $B_{i}$ de SeqQ, se $B_{i}$ é um invariante de $Y_{j}$, então, no Passo $13, j \leftarrow j+1$. Caso contrário, no Passo 07 ou 09, $i \leftarrow i+1$. Dessa forma, o número de iterações do algoritmo DECCON para encontrar a solução final $\mathrm{S}_{j}(A)$ é no máximo $i+j$, ou seja, $m_{s} \leq i+j$. Uma vez que existem no máximo $2^{9}$ subconjuntos do quadrado elementar, então $\mid$ SeqQ $\mid \leq 2^{9}$ e, conseqüentemente, $i \leq 2^{9}$.

Se $\left[X_{0}, X_{1}, \cdots, X_{j}\right]$ é a seqüência crescente atual de $A$ e se ela é maximal, então, pelas Proposições 5.15 e 5.16 e pelo Teorema $5.14, X_{j} \equiv A$ e $\operatorname{lower}\left(X_{j}\right)=j$. Logo, $j=\operatorname{lower}(A)$ e, pelo Teorema 4.18, $j=O(n)$. Portanto, $m_{s}=O(n)$.

Note que a seqüência SeqQ (no Passo 01) é construída previamente e, portanto, a construção dela não depende do EE de entrada.

Os passos cruciais para a complexidade de tempo do algoritmo são os Passos 05, 06, 08 e 12. A Subseção 5.6.1 apresenta a análise de complexidade dos Passos 05 e 06. A Subseção 5.6.2 fornece as complexidades dos Passos 08 e 12. Finalmente, na Subseção 5.6.3, mostramos a complexidade do algoritmo.

\subsubsection{Complexidade dos Passos 05 e 06}

Nos Passos 05 e 06 o algoritmo verifica se $\rho\left(Y_{j}\right)=(0,0)$ e se existe $k \in\{0,1, \cdots, 7\}$ tal que $\nu_{k}\left(Y_{j}\right)<\nu_{k}\left(B_{i}\right)$. Assim, nestes passos, o algoritmo tem que determinar $\rho\left(Y_{j}\right), \nu\left(Y_{j}\right)$ e $\nu\left(B_{i}\right)$.

O vetor projeção de cada elemento de SeqQ pode ser calculado antes da execução do algoritmo, uma vez que todos elementos nesta seqüência estão previamente fixados. Desta forma, a complexidade de tempo para determinar $\nu\left(B_{i}\right)$ e $\rho\left(B_{i}\right)$ é $O(1)$.

Para determinar o vetor projeção e o vetor retangular de $Y_{j}$, vamos considerar dois casos: $j=0$ or $j>0$. 
Se $j=0$, então o algoritmo tem que calcular o vetor projeção e o vetor retangular de $Y_{0}=A$. Um algoritmo $O\left(m_{p}\right)$ para determinar o "chain code" de $A$ (ou equivalentemente, o vetor projeção de $A$ ) pode ser visto em [36, p. 143]. Observe que o algoritmo precisa calcular $\nu(A)$ somente uma vez. Além disso, note que, se $\nu(A)$ já foi calculado anteriormente, então, pela definição do vetor retangular, a complexidade para determinar $\rho(A)$ é $O(1)$.

Portanto, se $j=0$, a complexidade de tempo do Passo 06, ou seja, para calcular $\nu\left(Y_{0}\right)$ e verificar se existe $k \in\{0,1, \cdots, 7\}$ tal que $\nu_{k}\left(Y_{0}\right)<\nu_{k}\left(B_{i}\right)$ é $O\left(m_{p}\right)$ e a complexidade do Passo 05, ou seja, para calcular $\rho\left(Y_{0}\right)$ e comparar se $\rho\left(Y_{0}\right)=(0,0)$ é $O(1)$.

No caso de $j>0$, o vetor projeção e o vetor retangular de $Y_{j}$ podem ser calculados usando o seguinte resultado.

Proposição 5.19 Sejam $B$ e $Y$ EE's. Se $B$ é um invariante de $Y$, então $\nu(Y \ominus B)=$ $\nu(Y)-\nu(B)$ e $\rho(Y \ominus B)=\rho(Y)-\rho(B)$.

Prova: Uma vez que $B$ é um invariante de $Y$, então $Y=(Y \ominus B) \oplus B$. Logo, pela Proposição 4.16, $\nu(Y)=\nu(Y \ominus B)+\nu(B)$ e portanto, $\nu(Y \ominus B)=\nu(Y)-\nu(B)$. De uma maneira similar, usando a Proposição 4.17, é fácil mostrar que $\rho(Y \ominus B)=\rho(Y)-\rho(B)$.

Se $\mathrm{S}_{j}(A)=\left[C_{1}, C_{2}, \cdots, C_{j}\right]$ é a solução parcial atual quando o EE considerado pelo algoritmo é $Y_{j}$, então $C_{j}$ é um invariante de $Y_{j-1}$ e o algoritmo construiu $Y_{j} \leftarrow Y_{j-1} \ominus C_{j}$. Assim, pela Proposição 5.19, $\nu\left(Y_{j}\right)=\nu\left(Y_{j-1}\right)-\nu\left(C_{j}\right)$ e $\rho\left(Y_{j}\right)=\rho\left(Y_{j-1}\right)-\rho\left(C_{j}\right)$. Se $j>0$, podemos considerar que o vetor projeção e o vetor retangular de $Y_{j-1}$ já foram construídos. Assim, neste caso, a complexidade de tempo para calcular $\nu\left(Y_{j}\right)$ e $\rho\left(Y_{j}\right)$ é $O(1)$, uma vez que $\nu\left(Y_{j}\right)$ e $\rho\left(Y_{j}\right)$ têm um número fixo de coordenadas, e o vetor retangular e o vetor projeção de cada $B \in$ SeqQ já foram determinados previamente antes de rodar o algoritmo.

Portanto, se $j>0$, a complexidade de tempo do Passo 05, ou seja, para calcular $\rho\left(Y_{j}\right)$ e comparar se $\rho\left(Y_{j}\right)=(0,0)$ é $O(1)$; e a complexidade do Passo 06, isto é, para calcular $\nu\left(Y_{j}\right)$ e verificar se existe $k \in\{0,1, \cdots, 7\}$ tal que $\nu_{k}\left(Y_{j}\right)<\nu_{k}\left(B_{i}\right)$ é $O(1)$.

\subsubsection{Complexidade dos Passos 08 e 12}

Para verificar se $B_{i}$ é um invariante de $Y_{j}$, temos que comparar se $Y_{j}=\left(Y_{j} \ominus B_{i}\right) \oplus B_{i}$. Dado um EE $X$, a complexidade para construir $X \oplus B_{i}$ ou $X \ominus B_{i}$, pela definição de adição e subtração de Minkowski, é $O\left(m_{x} \cdot m_{b}\right)$, onde $m_{x}$ e $m_{b}$ denotam, respectivamente, o número de pontos de $X$ e $B_{i}$. Uma vez que $B_{i}$ contém no máximo 9 pontos, então a complexidade para construir $X \oplus B_{i}$ ou $X \ominus B_{i}$ é $O\left(m_{x}\right)$.

Obviamente, pela definição da subtração de Minkowski, se $Y=A \ominus X$, então $|Y| \leq|A|$. Conseqüentemente, se $\left[Y_{0}, Y_{1}, \cdots, Y_{j}\right]$ é a seqüência decrescente atual de um dado $\mathrm{EE}$ 
$A$, então, para todo $k \in\{0,1, \cdots, j\},\left|Y_{k}\right| \leq|A|$. No Passo 08, o algoritmo constrói $Z_{j}=Y_{j} \ominus B_{i}$ e $W_{j}=Z_{j} \oplus B_{i}$; e depois verifica se $Y_{j}=W_{j}$. A complexidade para construir $Z_{j}=Y_{j} \ominus B_{i}$ e $W_{j}=Z_{j} \oplus B_{i}$ e comparar se $Y_{j}=W_{j}$ é $O\left(m_{p}\right)$, uma vez que $\left|Z_{j}\right| \leq\left|Y_{j}\right| \leq|A|=m_{p}$. Dessa forma, a complexidade do Passo 08 é $O\left(m_{p}\right)$. Além disso, a complexidade do Passo 12, ou seja, para construir $Y_{j+1}=Y_{j} \ominus B_{i}$, é $O\left(m_{p}\right)$, uma vez que $\left|Y_{j}\right| \leq|A|=m_{p}$.

\subsubsection{Complexidade do Algoritmo}

Pela Proposição 5.18, o número de iterações do algoritmo DeCCoN é $m_{s}=O(n)$. Assim, as comparações nos Passos 05, 06, 08 e 12 são executadas $m_{s}=O(n)$ vezes.

Uma vez que a primeira comparação no Passo 05 leva tempo $O\left(m_{p}\right)$ e as outras comparações levam tempo $O(1)$, então a complexidade total do algoritmo deste passo é $O\left(m_{p}\right)+\underbrace{O(1)+\cdots+O(1)}_{m_{s}-1 \text { times }}=O\left(m_{p}\right)+O\left(m_{s}\right)$.

Uma vez que cada comparação no Passo 06 leva tempo $O(1)$, então a complexidade total do algoritmo neste passo é $O\left(m_{s}\right)$.

Uma vez que cada comparação nos Passos 08 e 12 leva tempo $O\left(m_{p}\right)$, a complexidade total do algoritmo nestes passos é $O\left(m_{s} \cdot m_{p}\right)$.

Logo, a complexidade do algoritmo para encontrar uma decomposição ótima de um EE convexo $A$ é $O\left(m_{s} \cdot m_{p}\right)$, ou seja, $O\left(n^{3}\right)$, uma vez que $m_{s}=O(n)$ e $m_{p}=O\left(n^{2}\right)$.

\subsection{Conclusão}

Neste capítulo, apresentamos um algoritmo guloso para decompor um EE convexo em termos de um número mínimo de subconjuntos (não necessariamente convexos) do quadrado elementar. Este algoritmo é simples e inédito. A complexidade deste algoritmo é $O\left(n^{3}\right)$, onde $n$ é o valor máximo entre as duas coordenadas do vetor retangular do EE convexo de entrada.

Embora a complexidade de tempo do algoritmo do Xu [49] seja $O\left(n^{2}\right)$, todos elementos na decomposição encontrado por este algoritmo são convexos.

Logo, como a complexidade computacional dos algoritmos que implementam erosões e dilatações depende do número de pontos do EE, o algoritmo proposto neste capítulo tem uma vantagem sobre o algoritmo do Xu.

Uma outra qualidade de algoritmo proposto é que seu processo de otimização é muito simples, comparado ao algoritmo do Xu.

Um próximo passo deste trabalho pode ser fazer uma versão simplificada do algo- 
ritmo DecCon colocando-se na seqüencia SeqQ somente os elementos de SeqQ que são subconjuntos convexos do quadrado elementar.

Finalmente, uma análise teórica (prova de corretude e da complexidade de tempo) do algoritmo proposto foi apresentada.

\subsection{Apêndice do Capítulo 5}

Para facilitar a leitura deste capítulo e não distrair o leitor de seus resultados principais, apresentaremos as provas das Proposições 5.15 (na Subseção 5.8.1) e 5.16 (na Subseção 5.8.2) como um apêndice deste capítulo.

\subsubsection{Prova da Proposição 5.15}

Dividiremos a prova da Proposição 5.15 em duas partes. Na primeira, mostraremos alguns resultados intermediários necessários para esta prova e, na segunda parte, provaremos a Proposição 5.15 .

\subsubsection{Resultados Necessários para a Prova da Proposição 5.15}

Para prova da Proposição 5.15, precisamos de cinco resultados intermediários dados nos Lemas de 5.20 a 5.24 .

Lema 5.20 Seja $A$ um EE convexo. Sejam $\left[X_{0}, X_{1}, \cdots, X_{j}\right]$ e $S_{j}(A)=\left[C_{1}, C_{2}, \cdots, C_{j}\right]$ a seqüência crescente atual de $A$ e a solução parcial atual, respectivamente. Seja $D$ um elemento de SeqQ. Se, para algum $i \in\{1,2, \cdots, j\}, D$ é um invariante de $Y_{i-1}$, então $\rho_{0}\left(C_{i}\right)+\rho_{1}\left(C_{i}\right) \geq \rho_{0}(D)+\rho_{1}(D)$.

Prova: Seja $\left[Y_{0}, Y_{1}, \cdots, Y_{i-1}\right]$ a seqüência decrescente atual de $A$ quando $Y_{i-1}$ foi considerado pelo algoritmo. Uma vez que $D$ é um elemento de SeqQ e $D$ é um invariante de $Y_{i-1}$, então, pelo Lema 5.12, $D$ é um elemento de Restante[ $\left[Y_{i-1}\right]$.

Seja Restante $\left[Y_{i-1}\right]=\left[C_{i-1}, B_{r}, B_{r+1}, \cdots, B_{s-1}, C_{i}=B_{s}, B_{s+1}, \cdots, B_{n}\right]$. O algoritmo DecCon seleciona $C_{i}$ de Restante $\left[Y_{i-1}\right]$ para construir $\mathrm{S}_{i+1}(A) \leftarrow \mathrm{S}_{i}(A) \cdot\left[C_{i}\right]$, pois $C_{i}$ é o primeiro elemento de Restante $\left[Y_{i-1}\right]$ que é um invariante de $Y_{i-1}$, isto é, todos elementos da seqüência $\left[C_{i-1}, B_{r}, B_{r+1}, \cdots, B_{s-1}\right]$ não são invariantes de $Y_{i-1}$. Assim, uma vez que $D$ é também um invariante de $Y_{i-1}$ e $D$ é também um elemento de Restante[ $\left[Y_{i-1}\right]$, então $D$ deve ser um elemento de Restante $\left[Y_{i}\right]=\left[C_{i}=B_{s}, B_{s+1}, \cdots, B_{n}\right]$. Logo, pela ordem escolhida para construir a seqüência SeqQ, $\rho_{0}\left(C_{i}\right)+\rho_{1}\left(C_{i}\right) \geq \rho_{0}(D)+\rho_{1}(D)$. 
O Lema 5.21 fornece um resultado simples e interessante sobre a adição de Minkowski entre o último elemento da seqüência decrescente atual e o último elemento da correspondente solução parcial. Este lema é simplesmente um passo intermediário para provar o Lema 5.22 .

Lema 5.21 Seja $A$ um EE convexo. Se $\left[Y_{0}, Y_{1}, \cdots, Y_{j}\right]$ e $\mathrm{S}_{j}(A)=\left[C_{1}, C_{2}, \cdots, C_{j}\right]$ são, respectivamente, a seqüência decrescente atual de $A$ e a solução parcial atual, então $Y_{j-1}=$ $Y_{j} \oplus C_{j}$.

Prova: Uma vez que $\mathrm{S}_{j}(A)=\left[C_{1}, C_{2}, \cdots, C_{j}\right]$ é a solução parcial atual, então $Y_{j}=$ $Y_{j-1} \ominus C_{j}$ e $C_{j}$ é um invariante $Y_{j-1}$. Assim, uma vez que $C_{j}$ é um invariante $Y_{j-1}$, então $Y_{j-1}=\left(Y_{j-1} \ominus C_{j}\right) \oplus C_{j}$, e, conseqüentemente, como $Y_{j}=Y_{j-1} \ominus C_{j}$, então $Y_{j-1}=Y_{j} \oplus C_{j}$.

Como uma conseqüência do Lema 5.21, o próximo resultado fornece uma propriedade da seqüência decrescente e a solução parcial atuais. Este lema é somente um passo intermediário para provar o Lema 5.23.

Lema 5.22 Seja A um EE convexo. Seja $\left[Y_{0}, Y_{1}, \cdots, Y_{j}\right]$ a seqüência decrescente atual de $A$. Se $\mathrm{S}_{j}(A)=\left[C_{1}, C_{2}, \cdots, C_{j}\right]$ é a solução parcial atual, então, para todo $1 \leq \ell \leq j$, $Y_{\ell-1}=Y_{j} \oplus C_{j} \oplus C_{j-1} \oplus \cdots \oplus C_{\ell}$.

Prova: Provaremos este lema por indução em $\ell$. Se $\ell=j$, então, pelo Lema 5.21, $Y_{j-1}=Y_{j} \oplus C_{j}$. Suponha, por hipótese de indução, que este lema vale para $1<\ell \leq j$, ou seja, $Y_{\ell-1}=Y_{j} \oplus C_{j} \oplus C_{j-1} \oplus \cdots \oplus C_{\ell}$. Provaremos que $Y_{\ell-2}=Y_{j} \oplus C_{j} \oplus \cdots \oplus C_{\ell} \oplus C_{\ell-1}$.

Se $\mathrm{S}_{\ell-1}(A)=\left[C_{1}, C_{2}, \cdots, C_{\ell-1}\right]$ é a solução parcial atual quando $Y_{\ell-1}$ foi o EE considerado pelo algoritmo, então, pelo Lema 5.21, $Y_{\ell-2}=Y_{\ell-1} \oplus C_{\ell-1}$. Uma vez que, por hipótese de indução, $Y_{\ell-1}=Y_{j} \oplus C_{j} \oplus C_{j-1} \oplus \cdots \oplus C_{\ell}$, então, $Y_{\ell-2}=\left(Y_{j} \oplus C_{j} \oplus C_{j-1} \oplus \cdots \oplus C_{\ell}\right) \oplus C_{\ell-1}$.

Lema 5.23 Seja $A$ um EE convexo. Seja $\left[Y_{0}, Y_{1}, \cdots, Y_{j}\right]$ e $\mathrm{S}_{j}(A)=\left[C_{1}, C_{2}, \cdots, C_{j}\right]$, respectivamente, a seqüência decrescente atual de $A$ e a solução parcial atual com $j>2$. Sejam $C_{i}$ e $C_{k}$ dois elementos de $\mathrm{S}_{j}(A)$ tais que $i<k$. Se $T$ é um EE tal que $|T| \geq 2$, $\rho(T) \leq(2,2)$ e $T$ é um invariante de $C_{i} \oplus C_{k}$ ou $C\left(C_{i}\right) \oplus C\left(C_{k}\right)$, então existe um EE D em SeqQ tal que $\rho(D)=\rho(T)$ e $D$ é im invariante de $Y_{i-1}$.

Prova: Uma vez que $|T| \geq 2, \rho(T) \leq(2,2)$, então, claramente, existe um elemento $D$ em SeqQ tal que $D \equiv T$. Assim, evidentemente, $\rho(D)=\rho(T)$. Falta mostrar que $D$ (ou equivalentemente, $T$ ) é um invariante de $Y_{i-1}$.

Uma vez que $T$ é um invariante de $C_{i} \oplus C_{k}$ ou $C\left(C_{i}\right) \oplus C\left(C_{k}\right)$, se provarmos que $C_{i} \oplus C_{k}$ e $C\left(C_{i}\right) \oplus C\left(C_{k}\right)$ são invariantes de $Y_{i-1}$, então, pela propriedade de transitividade de 


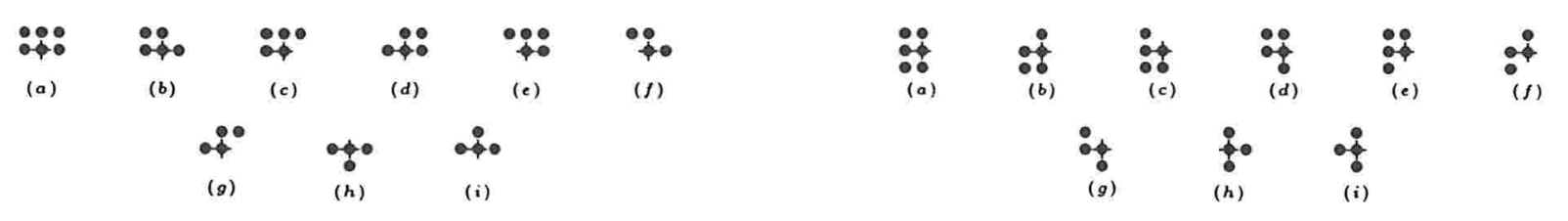

Figura 5.3: Elementos de $\mathcal{R}$.

Figura 5.4: Elementos de $\mathcal{S}$.

invariança (dada pela Proposição 5.2), $T$ é um invariante de $Y_{i-1}$ e, conseqüentemente, $D$ também é um invariante de $Y_{i-1}$.

Agora, provamos que $C_{i} \oplus C_{k}$ é um invariante de $Y_{i-1}$.

Sejam $\left[Y_{0}, Y_{1}, \cdots, Y_{i}\right]$ e $S_{i}(A)=\left[C_{1}, C_{2}, \cdots, C_{i}\right]$, respectivamente, a seqüência decrescente atual de $A$ e a solução parcial atual quando $Y_{i}$ foi o EE considerado pelo algoritmo. Uma vez que, $1 \leq i \leq j$, pelo Lema $5.22, Y_{i-1}=Y_{j} \oplus C_{j} \oplus C_{j-1} \oplus \cdots \oplus C_{i}$. Note que $C_{i}$ e $C_{k}$ são elementos em $\left[C_{i}, C_{i+1}, \cdots, C_{j}\right]$, uma vez que $i<k \leq j$. Se $Z$ é a adição de Minkowski de todos elementos em $\left[C_{i}, C_{i+1}, \cdots, C_{j}\right]$ exceto para $C_{i}$ e $C_{k}$, então $Y_{i-1}=Y_{j} \oplus Z \oplus C_{i} \oplus C_{k}=\left(Y_{j} \oplus Z\right) \oplus\left(C_{i} \oplus C_{k}\right)$. Logo, pela Proposição $3.15, C_{i} \oplus C_{k}$ é um invariante de $Y_{i-1}$.

Falta provar que $C\left(C_{i}\right) \oplus C\left(C_{k}\right)$ é um invariante de $Y_{i-1}$.

Seja $\left[X_{0}, X_{1}, \cdots, X_{i-1}\right]$ a seqüência crescente atual de $A$ quando $Y_{i-1}$ foi o EE considerado pelo algoritmo. Uma vez que, pelo Lema 5.8, $Y_{i-1}=A \ominus X_{i-1}$ e, pelo Lema 5.9, $X_{i-1}$ é um invariante de $A$, então, pelo Lema 5.6, $Y_{i-1}$ é um EE convexo. Assim, uma vez que $Y_{i-1}=Y_{j} \oplus Z \oplus C_{i} \oplus C_{k}$, então, pelo Lema 5.4, $Y_{i-1}=Y_{j} \oplus Z \oplus C\left(C_{i}\right) \oplus C_{k}=$ $Y_{j} \oplus Z \oplus C\left(C_{i}\right) \oplus C\left(C_{k}\right)$. Dessa forma, pela Proposição 3.15, $C\left(C_{i}\right) \oplus C\left(C_{k}\right)$ é um invariante de $Y_{i-1}$.

Lema 5.24 Seja $A$ um EE convexo. Sejam $\left[Y_{0}, Y_{1}, \cdots, Y_{j}\right]$ e $\mathrm{S}_{j}(A)=\left[C_{1}, C_{2}, \cdots, C_{j}\right]$, respectivamente, a seqüência decrescente atual de $A$ e a solução parcial atual. Se existem $C_{i}$ e $C_{k}$ em $\mathrm{S}_{j}(A)$ tais que $\rho\left(C_{i}\right)=(2,1)$ e $\rho\left(C_{k}\right)=(1,2)$, então existe $D$ em SeqQ tal que $\rho(D)=(2,2)$ e $D$ é um invariante de $Y_{\ell-1}$, onde $\ell=\min \{i, k\}$.

Prova: Sejam $\mathcal{R}$ e $\mathcal{S}$ os conjuntos de todos elementos em SeqQ tais que os vetores retangulares sejam iguais a, respectivamente, $(2,1)$ e $(1,2)$. Uma vez que $\rho\left(C\left(C_{i}\right)\right)=$ $\rho\left(C_{i}\right)=(2,1)$ e $\rho\left(C\left(C_{k}\right)\right)=\rho\left(C_{k}\right)=(1,2)$, então $C\left(C_{i}\right) \in \mathcal{R}$ e $C\left(C_{k}\right) \in \mathcal{S}$.

Cada subconjunto convexo do quadrado elementar apresentado na Figura 5.3 (respectivamente, Figura 5.4) é equivalente sob translação a um elemento of $\mathcal{R}$ (respectivamente, $\mathcal{S}$ ) e vice-versa. Assim, sem perda de generalidade, suponha que os elementos de $\mathcal{R}$ (respectivamente, $\mathcal{S}$ ) sejam os EE's convexos apresentados na Figura 5.3 (respectivamente, Figura 5.4). 


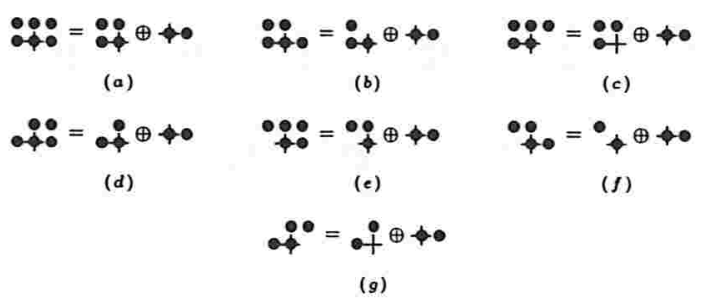

Figura 5.5: Todos elementos de $\mathcal{R}_{1}$ são decomponíveis.

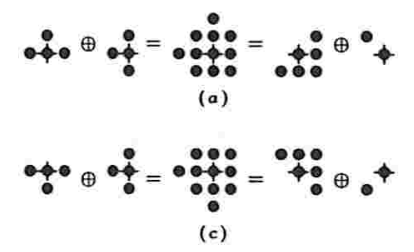

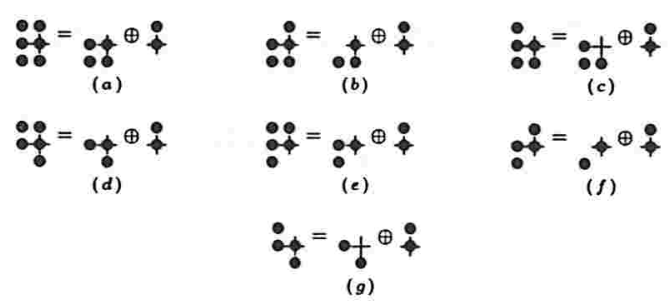

Figura 5.6: Todos elementos de $\mathcal{S}_{1}$ são decomponíveis.

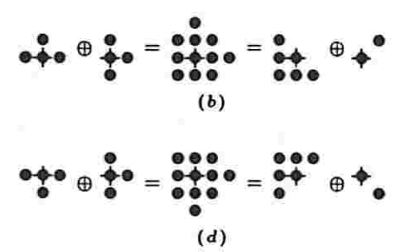

Figura 5.7: Todas possibilidades de $C\left(B_{i}\right) \oplus C\left(B_{k}\right)$ se $C\left(B_{i}\right) \in \mathcal{R}_{2}$ e $C\left(B_{k}\right) \in \mathcal{S}_{2}$.

Agora, particionamos os conjuntos $\mathcal{R}$ e $\mathcal{S}$ da seguinte forma. Sejam $\mathcal{R}_{1}$ e $\mathcal{S}_{1}$ os conjuntos formados pelos EE's convexos apresentados, respectivamente, nas Figuras 5.3.a a 5.3.g e nas Figuras 5.4.a a 5.4.g. Sejam $\mathcal{R}_{2}=\mathcal{R}-\mathcal{R}_{1}$ e $\mathcal{S}_{2}=\mathcal{S}-\mathcal{S}_{1}$.

Uma vez que $C\left(C_{i}\right) \in \mathcal{R}, C\left(C_{k}\right) \in \mathcal{S}$ e $\left\{\mathcal{R}_{1}, \mathcal{R}_{2}\right\}$ e $\left\{\mathcal{S}_{1}, \mathcal{S}_{2}\right\}$ são partições, respectivamente, de $\mathcal{R}$ e $\mathcal{S}$, então podemos considerar somente dois casos: (1) $C\left(C_{i}\right) \in \mathcal{R}_{1}$ ou $C\left(C_{k}\right) \in \mathcal{S}_{1}$; e (2) $C\left(C_{i}\right) \in \mathcal{R}_{2}$ e $C\left(C_{i}\right) \in \mathcal{S}_{2}$. Note que, o Caso 2 é satisfeito se, e somente se, o Caso 1 não é satisfeito.

Caso 1: $C\left(C_{i}\right) \in \mathcal{R}_{1}$ ou $C\left(C_{k}\right) \in \mathcal{S}_{1}$.

Consideraremos que $C\left(C_{i}\right) \in \mathcal{R}_{1}$ (a prova para $C\left(C_{k}\right) \in \mathcal{S}_{1}$ pode ser feita de maneira similar).

Como indicado na Figura 5.5, se $C\left(C_{i}\right) \in \mathcal{R}_{1}$, então existem dois EE's convexos $D_{1}$ e $D_{2}$ tais que $C\left(C_{i}\right)=D_{1} \oplus D_{2}, \rho\left(D_{1}\right)=(1,1)$ e $\rho\left(D_{2}\right)=(1,0)$. Dessa forma, $C\left(C_{i}\right) \oplus C\left(C_{k}\right)=\left(D_{1} \oplus D_{2}\right) \oplus C\left(C_{k}\right)=D_{1} \oplus\left(D_{2} \oplus C\left(C_{k}\right)\right)=D_{1} \oplus T$, onde $T=D_{2} \oplus C\left(C_{k}\right)$. Assim, uma vez que $T$ é um invariante de $C\left(C_{i}\right) \oplus C\left(C_{k}\right)$ (pela Proposição 3.15) e $\rho(T)=\rho\left(D_{2}\right)+\rho\left(C\left(C_{k}\right)\right)=(2,2)$ (pela Proposição 4.17), então, pelo Lema 5.23, existe um elemento $D$ em SeqQ tal que $\rho(D)=\rho(T)=(2,2)$ e $D$ é um invariante de $Y_{i-1}($ se $i<k)$ ou $Y_{k-1}$ (se $\left.k<i\right)$.

Caso 2: $C\left(C_{i}\right) \in \mathcal{R}_{2}$ e $C\left(C_{i}\right) \in \mathcal{S}_{2}$.

Neste caso, a Figura 5.7 apresenta todas possibilidades de $C\left(C_{i}\right) \oplus C\left(C_{k}\right)$. Assim, como indicado nesta figura, existem dois EE's convexos $D_{1}$ e $D_{2}$ tais que $C\left(C_{i}\right) \oplus$ $C\left(C_{k}\right)=D_{1} \oplus D_{2}, \rho\left(D_{1}\right)=(2,2)$ e $\rho\left(D_{2}\right)=(1,1)$. Assim, pela Proposição $3.15, D_{1}$ 
é um invariante de $C\left(C_{i}\right) \oplus C\left(C_{k}\right)$. Dessa forma, pelo Lema 5.23, existe $D$ em SeqQ tal que $\rho(D)=\rho\left(D_{1}\right)=(2,2)$ e $D$ é um invariante de $Y_{i-1}\left(\right.$ se $i<k$ ) ou $Y_{k-1}$ (se $k<i)$.

Portanto, existe $D$ em SeqQ tal que $\rho(D)=(2,2)$ e $D$ é um invariante de $Y_{\ell-1}$, onde $\ell=\min \{i, k\}$.

Agora, usando os resultados dos Lemas de 5.20 a 5.24, podemos provar a Proposição 5.15 .

\subsubsection{Demonstração da Proposição 5.15}

Seja $\mathrm{S}_{j}(A)=\left[C_{1}, C_{2}, \cdots, C_{j}\right]$ a solução parcial atual. Obviamente, por construção, $\mathrm{S}_{j}(A)$ é a seqüência geradora de $\left[X_{0}, X_{1}, \cdots, X_{j}\right]$. Assim, para provar que $\left[X_{0}, X_{1}, \cdots, X_{j}\right]$ é uma seqüência elemento retangular de $A$, basta mostrar que sua seqüência geradora $S_{j}(A)$ é uma seqüência retangular. Dessa forma, temos que provar que as Restrições da definição de seqüência retangular são válidas para $\mathrm{S}_{j}(A)$.

Prova que as Restrições de $(a)$ a $(f)$ são válidas:

Suponha, por absurdo, que uma das Restrições de $(a)$ a $(f)$ da definição de seqüência retangular não seja válida para $\mathrm{S}_{j}(A)$. Assim, existem dois elementos $C_{i}$ e $C_{k}, \operatorname{com} i<k$, em $\mathrm{S}_{j}(A)=\left[C_{1}, C_{2}, \cdots, C_{j}\right]$ tais que $\rho\left(C_{i} \oplus C_{k}\right)=\rho\left(C_{i}\right)+\rho\left(C_{k}\right) \leq(2,2)$. Seja $T=C_{i} \oplus C_{k}$. Claramente, $T$ é um invariante de $C_{i} \oplus C_{k}$. Além disso, como $C_{i}$ e $C_{k}$ são elementos de SeqQ, então $|T| \geq 2$ e $\rho_{0}\left(C_{k}\right)+\rho_{1}\left(C_{k}\right)>0$.

Uma vez que $\rho(T)=\rho\left(C_{i}\right)+\rho\left(C_{k}\right) \leq(2,2),|T| \geq 2$ e $T$ é um invariante de $C_{i} \oplus C_{k}$, então, pelo Lema 5.23, existe um elemento $D$ em SeqQ tal que $\rho(D)=\rho(T)$ e $D$ é um invariante de $Y_{i-1}$. Assim, uma vez que $i \in\{1,2, \cdots, j\}$, então, pelo Lema 5.20, $\rho_{0}\left(C_{i}\right)+\rho_{1}\left(C_{i}\right) \geq \rho_{0}(D)+\rho_{1}(D)$.

Uma vez que $\rho(D)=\rho(B)=\rho\left(C_{i}\right)+\rho\left(C_{k}\right)$, então

$$
\begin{aligned}
\rho_{0}\left(C_{i}\right)+\rho_{1}\left(C_{i}\right) \geq \rho_{0}(D)+\rho_{1}(D) & =\left(\rho_{0}\left(C_{i}\right)+\rho_{0}\left(C_{k}\right)\right)+\left(\rho_{1}\left(C_{i}\right)+\rho_{1}\left(C_{k}\right)\right) \\
& =\left(\rho_{0}\left(C_{i}\right)+\rho_{1}\left(C_{i}\right)\right)+\left(\rho_{0}\left(C_{k}\right)+\rho_{1}\left(C_{k}\right)\right) .
\end{aligned}
$$

Dessa forma, $\rho_{0}\left(C_{k}\right)+\rho_{1}\left(C_{k}\right) \leq 0$. Mas isto é um contradição, pois $\rho_{0}\left(C_{k}\right)+\rho_{1}\left(C_{k}\right)>0$. Portanto, todas as Restrições de $(a)$ a $(f)$, são válidas para $\mathrm{S}_{j}(A)$.

Prova que as Restrições de $(g)$ a $(j)$ são válidas:

Suponha por absurdo que uma das restrições, de $(g)$ a $(j)$, não seja válida. Dessa forma, $c_{2,0}\left(\mathrm{~S}_{j}(A)\right)>0$ ou $c_{0,2}\left(\mathrm{~S}_{j}(A)\right)>0$. Conseqüentemente, existe um elemento $C_{k} \mathrm{em}$ $\mathrm{S}_{j}(A)$ tal que $\rho\left(C_{k}\right)=(2,0)$ ou $\rho\left(C_{k}\right)=(0,2)$. Consideraremos o caso $\rho\left(C_{k}\right)=(2,0)$. O outro caso $\rho\left(C_{k}\right)=(0,2)$ pode ser provado de uma maneira similar. Logo, pelas restrições $(g)$ ou $(h), c_{1,1}\left(\mathrm{~S}_{j}(A)\right)>0$ ou $c_{1,2}\left(\mathrm{~S}_{j}(A)\right)>0$, e, portanto, existe um elemento $C_{i}$ em $\mathrm{S}_{j}(A)$ tal que $\rho\left(C_{i}\right)=(1,1)$ ou $\rho\left(C_{i}\right)=(1,2)$. Uma vez que $\rho_{0}\left(C_{i}\right)+\rho_{1}\left(C_{i}\right) \geq \rho_{0}\left(C_{k}\right)+\rho_{1}\left(C_{k}\right)$, 
pela ordem escolhida para construir SeqQ, podemos considerar que $i<k$ (o caso $i>k$ é analógo).

Uma vez que, $\rho\left(C\left(C_{k}\right)\right)=\rho\left(C_{k}\right)=(2,0)$ (pela definição de vetor retangular), então $C\left(C_{k}\right)$ é uma linha horizontal com três pontos consecutivos. Dessa forma, existem duas linhas horizontais com dois pontos consecutivos cada um, digamos $L_{1}$ e $L_{2}$, tais que $C\left(C_{k}\right)=L_{1} \oplus L_{2}$. Claramente, $\rho\left(L_{1}\right)=\rho\left(L_{2}\right)=(1,0)$.

Assim, $C\left(C_{i}\right) \oplus C\left(C_{k}\right)=C\left(C_{i}\right) \oplus\left(L_{1} \oplus L_{2}\right)=\left(C\left(C_{i}\right) \oplus L_{1}\right) \oplus L_{2}$. Dessa forma, pela Proposição 3.15, T=C(C $\left.C_{i}\right) \oplus L_{1}$ é um invariante de $C\left(C_{i}\right) \oplus C\left(C_{k}\right)$. Além disso, pela Proposição 4.17, $\rho(T)=\rho\left(C\left(C_{i}\right)\right)+\rho\left(L_{1}\right) \leq(2,2)$, uma vez que $\rho\left(C_{i}\right)=(1,1)$ ou $\rho\left(C_{i}\right)=(1,2)$.

Uma vez que, $\rho(T) \leq(2,2), B$ é um invariante de $C\left(C_{i}\right) \oplus C\left(C_{k}\right)$ e $i<k$, então, pelo Lema 5.23, existe $D$ em SeqQ tal que $\rho(D)=\rho(T)$ e $D$ é um invariante de $Y_{i-1}$. Dessa forma, uma vez que $i \in\{1,2, \cdots, j\}$, pelo Lema 5.20, $\rho_{0}\left(C_{i}\right)+\rho_{1}\left(C_{i}\right) \geq \rho_{0}(D)+\rho_{1}(D)$.

Uma vez que $\rho(D)=\rho(B)=\rho\left(C\left(C_{i}\right)\right)+\rho\left(L_{1}\right)$ e $\rho\left(C\left(C_{i}\right)\right)=\rho\left(C_{i}\right)$, então

$$
\begin{aligned}
\rho_{0}\left(C_{i}\right)+\rho_{1}\left(C_{i}\right) \geq \rho_{0}(D)+\rho_{1}(D) & =\left(\rho_{0}\left(C\left(C_{i}\right)\right)+\rho_{0}\left(L_{1}\right)\right)+\left(\rho_{1}\left(C\left(C_{i}\right)\right)+\rho_{1}\left(L_{1}\right)\right) \\
& =\left(\rho_{0}\left(C_{i}\right)+\rho_{0}\left(L_{1}\right)\right)+\left(\rho_{1}\left(C_{i}\right)+\rho_{1}\left(L_{1}\right)\right) \\
& =\left(\rho_{0}\left(C_{i}\right)+\rho_{1}\left(C_{i}\right)\right)+\left(\rho_{0}\left(L_{1}\right)+\rho_{1}\left(L_{1}\right)\right) .
\end{aligned}
$$

Assim, $\rho_{0}\left(L_{1}\right)+\rho_{1}\left(L_{1}\right) \leq 0$. Mas isto contradiz o fato de que $\rho\left(L_{1}\right)=(1,0)$. Logo, as Restrições de $(g)$ a $(j)$ são válidas para $\mathrm{S}_{j}(A)$.

Prova que a Restrição $(k)$ é válida:

Suponha por absurdo que a Restrição $(k)$ não seja válida, ou seja, $c_{1,2}\left(\mathrm{~S}_{j}(A)\right)>0$ e $c_{2,1}\left(\mathrm{~S}_{j}(A)\right)>0$. Sejam $C_{i}$ e $C_{k}$ dois elementos em $\mathrm{S}_{j}(A)=\left[C_{1}, C_{2}, \cdots, C_{j}\right]$ tais que, $\rho\left(C_{i}\right)=(1,2)$ e $\rho\left(C_{k}\right)=(2,1)$. Sem perda de generalidade suponha que $i<k$.

Pelo Lema 5.24, existe um elemento $D$ em SeqQ tal que $\rho(D)=(2,2)$ e $D$ é um invariante de $Y_{i-1}$. Dessa forma, uma vez que $i \in\{1,2, \cdots, j\}$, pelo Lema $5.20, \rho_{0}\left(C_{i}\right)+$ $\rho_{1}\left(C_{i}\right) \geq \rho_{0}(D)+\rho_{1}(D)$. Mas, isto contradiz o fato de que $\rho_{0}\left(C_{i}\right)+\rho_{1}\left(C_{i}\right)=3$ e $\rho_{0}(D)+$ $\rho_{1}(D)=4$. Logo, $c_{1,2}\left(\mathrm{~S}_{j}(A)\right)=0$ ou $c_{2,1}\left(\mathrm{~S}_{j}(A)\right)=0$ e, portanto, $c_{2,1}\left(\mathrm{~S}_{j}(A)\right) \cdot c_{1,2}\left(\mathrm{~S}_{j}(A)\right)=$ 0 .

\subsubsection{Prova da Proposição 5.16}

Nesta subseção, daremos a prova da Proposição 5.16. Mas, antes isso, precisamos do resultado do seguinte lema.

Lema 5.25 Seja A um EE. Seja $\mathrm{E}=\left[X_{0}, X_{1}, \cdots, X_{j}\right]$ uma seqüência elemento retangular de $A$ e seja $\mathrm{R}=\left[C_{1}, C_{2}, \cdots, C_{j}\right]$ uma seqüência retangular geradora de $\mathrm{E}$. Considere as seguintes condições.

(a) $\rho_{0}\left(X_{j-1}\right) \geq \rho_{1}\left(X_{j-1}\right), \rho_{0}\left(C_{j}\right) \geq \rho_{1}\left(C_{j}\right)$ e $\rho_{0}\left(C_{j}\right)=2$; 
(b) $\rho_{0}\left(X_{j-1}\right) \geq \rho_{1}\left(X_{j-1}\right), \rho_{0}\left(C_{j}\right) \geq \rho_{1}\left(C_{j}\right), \rho_{0}\left(C_{j}\right)=1$ e $\rho_{0}\left(X_{j-1}\right)$ é um número par;

(c) $\rho_{1}\left(X_{j-1}\right) \geq \rho_{0}\left(X_{j-1}\right), \rho_{1}\left(C_{j}\right) \geq \rho_{0}\left(C_{j}\right)$ e $\rho_{1}\left(C_{j}\right)=2$;

(d) $\rho_{1}\left(X_{j-1}\right) \geq \rho_{0}\left(X_{j-1}\right), \rho_{1}\left(C_{j}\right) \geq \rho_{0}\left(C_{j}\right), \rho_{1}\left(C_{j}\right)=1$ e $\rho_{1}\left(X_{j-1}\right)$ é um número par;

Se uma das condições acima é satisfeita, então lower $\left(X_{j}\right)=\operatorname{lower}\left(X_{j-1}\right)+1$.

Prova: Pela definição de seqüência elemento retangular de $A, X_{j}=X_{j-1} \oplus C_{j}$. Dessa forma, pela Proposição 4.17, $\rho\left(X_{j}\right)=\rho\left(X_{j-1}\right)+\rho\left(C_{j}\right)$. Pelo Teorema 4.18, temos que lower $\left(X_{j}\right)=\left\lceil\max \left\{\rho_{0}\left(X_{j}\right), \rho_{1}\left(X_{j}\right)\right\} / 2\right\rceil$. Logo, uma vez que $\rho\left(X_{j}\right)=\rho\left(X_{j-1}\right)+\rho\left(C_{j}\right)$, então lower $\left(X_{j}\right)=\left\lceil\max \left\{\rho_{0}\left(X_{j-1}\right)+\rho_{0}\left(C_{j}\right), \rho_{1}\left(X_{j-1}\right)+\rho_{1}\left(C_{j}\right)\right\} / 2\right\rceil$.

Portanto, se a Condição $(a)$ ou $(b)$ é satisfeita, então, o limite inferior lower $\left(X_{j}\right)=$ $\left\lceil\left(\rho_{0}\left(X_{j-1}\right)+\rho_{0}\left(C_{j}\right)\right) / 2\right\rceil$, uma vez que $\rho_{0}\left(X_{j-1}\right) \geq \rho_{1}\left(X_{j-1}\right)$ e $\rho_{0}\left(C_{j}\right) \geq \rho_{1}\left(C_{j}\right)$. Além disso, pelo Teorema 4.18 , lower $\left(X_{j-1}\right)=\left\lceil\rho_{0}\left(X_{j-1}\right) / 2\right\rceil$, uma vez que $\rho_{0}\left(X_{j-1}\right) \geq \rho_{1}\left(X_{j-1}\right)$.

Assim, se a Condição $(a)$ é satisfeita, então,

$$
\begin{aligned}
\text { lower }\left(X_{j}\right) & =\left\lceil\left(\rho_{0}\left(X_{j-1}\right)+\rho_{0}\left(C_{j}\right)\right) / 2\right\rceil \\
& \left.=\left\lceil\left(\rho_{0}\left(X_{j-1}\right)+2\right) / 2\right\rceil \quad \text { (uma vez que } \rho_{0}\left(C_{j}\right)=2\right) . \\
& =\left\lceil\rho_{0}\left(X_{j-1}\right) / 2\right\rceil+1 \quad \\
& =\text { lower }\left(X_{j-1}\right)+1 \\
&
\end{aligned}
$$

No caso em que a Condição $(b)$ é satisfeita,

$$
\begin{aligned}
\text { lower }\left(X_{j}\right) & =\left\lceil\left(\rho_{0}\left(X_{j-1}\right)+\rho_{0}\left(C_{j}\right)\right) / 2\right\rceil \\
& =\left\lceil\left(\rho_{0}\left(X_{j-1}\right)+1\right) / 2\right\rceil
\end{aligned}
$$

Se a Condição $(c)$ ou $(d)$ é satisfeita, então, o limite inferior lower $\left(X_{j}\right)=\left\lceil\left(\rho_{1}\left(X_{j-1}\right)+\right.\right.$ $\left.\left.\rho_{1}\left(C_{j}\right)\right) / 2\right\rceil$, uma vez que $\rho_{1}\left(X_{j-1}\right) \geq \rho_{0}\left(X_{j-1}\right)$ e $\rho_{1}\left(C_{j}\right) \geq \rho_{0}\left(C_{j}\right)$. Além disso, pelo Teorema 4.18 , lower $\left(X_{j-1}\right)=\left\lceil\rho_{1}\left(X_{j-1}\right) / 2\right\rceil$, uma vez que $\rho_{1}\left(X_{j-1}\right) \geq \rho_{0}\left(X_{j-1}\right)$.

Assim, se a Condição $(c)$ ou $(d)$ é satisfeita, o resultado lower $\left(X_{j}\right)=\operatorname{lower}\left(X_{j-1}\right)+1$ pode ser provado de uma maneira análoga à prova das Condições $(a)$ e $(b)$, respectivamente, substituindo-se $\rho_{0}\left(X_{j-1}\right)$ por $\rho_{1}\left(X_{j-1}\right)$ e $\rho_{0}\left(C_{j}\right)$ por $\rho_{1}\left(C_{j}\right)$.

Seja $A$ um EE. Seja $\mathrm{E}=\left[X_{0}, X_{1}, \cdots, X_{j}\right]$ uma seqüência elemento retangular de $A$. Se $\mathrm{R}=\left[C_{1}, C_{2}, \cdots, C_{j}\right]$ é uma seqüência retangular geradora de $\mathrm{E}$, então, pela definição 
de seqüência elemento retangular, R é uma decomposição seqüencial de $X_{j}$, ou seja, $X_{j}=$ $C_{1} \oplus C_{2} \oplus \cdots \oplus C_{j}$. Dessa forma, pela Proposição 4.17, $\rho\left(X_{j}\right)=\rho\left(C_{1}\right)+\rho\left(C_{2}\right)+\cdots+\rho\left(C_{j}\right)$. Logo, uma vez que $c_{x, y}(\mathrm{R})$ é o número de EE's em $\mathrm{R}$ cujos vetores retangulares são iguais a $(x, y)$, então, obviamente,

$$
\rho\left(X_{j}\right)=\sum_{x=0}^{2} \sum_{y=0}^{2} c_{x, y}(\mathrm{R}) \cdot(x, y) .
$$

Portanto, cada coordenada de $\rho\left(X_{j}\right)$ pode ser escrita como

$$
\begin{aligned}
& \rho_{0}\left(X_{j}\right)=2\left[c_{2,2}(\mathrm{R})+c_{2,1}(\mathrm{R})+c_{2,0}(\mathrm{R})\right]+c_{1,2}(\mathrm{R})+c_{1,1}(\mathrm{R})+c_{1,0}(\mathrm{R}) ; \\
& \rho_{1}\left(X_{j}\right)=2\left[c_{2,2}(\mathrm{R})+c_{1,2}(\mathrm{R})+c_{0,2}(\mathrm{R})+c_{2,1}(\mathrm{R})+c_{1,1}(\mathrm{R})+c_{0,1}(\mathrm{R}) .\right.
\end{aligned}
$$

Dado um EE, seja $\mathrm{R}=\left[C_{1}, C_{2}, \cdots, C_{j}\right]$ uma seqüência retangular de $A$. Se $\rho_{0}\left(C_{j}\right)+$ $\rho_{1}\left(C_{j}\right)=x$, então, pela ordem escolhida para construir SeqQ (e, conseqüentemente, Seqlnv $[A])$, para todo elemento $C_{i}$ de $\mathrm{R}, \rho_{0}\left(C_{i}\right)+\rho_{1}\left(C_{i}\right) \geq x$. Assim, por exemplo, se $\rho_{0}\left(C_{j}\right)+\rho_{1}\left(C_{j}\right)=3$, então, para todo elemento $C_{i}$ de $\mathrm{R}, \rho_{0}\left(C_{i}\right)+\rho_{1}\left(C_{i}\right) \geq 3$. Dessa forma, neste mesmo exemplo, $c_{0,2}(\mathrm{R})=c_{2,0}(\mathrm{R})=c_{1,1}(\mathrm{R})=c_{0,1}(\mathrm{R})=c_{1,0}(\mathrm{R})=0$. Além disso, se $\left[X_{0}, X_{1}, \cdots, X_{j}\right]$ é a seqüência elemento retangular gerada por $\mathrm{R}$, então, pelas Igualda$\operatorname{des}(1)$ e $(2), \rho_{0}\left(X_{j}\right)=2\left[c_{2,2}(\mathrm{R})+c_{2,1}(\mathrm{R})\right]+c_{1,2}(\mathrm{R})$ e $\rho_{1}\left(X_{j}\right)=2\left[c_{2,2}(\mathrm{R})+c_{1,2}(\mathrm{R})\right]+c_{2,1}(\mathrm{R})$.

Agora, usando os fatos discutidos acima, podemos provar o fato de que, dado um EE $A$, para qualquer seqüência elemento retangular $\left[X_{0}, X_{1}, \cdots, X_{j}\right]$ de $A$, temos que lower $\left(X_{j}\right)=j$.

\subsubsection{Prova da Proposição 5.16}

Provaremos este teorema por indução em $j$. Quando $j=0$, é óbvio que $\operatorname{lower}\left(X_{0}\right)=$ $\lceil\max \{0,0\} / 2\rceil=0$, uma vez que $X_{0}$ é o conjunto unitário que contém a origem. Suponha, por hipótese de indução, que este teorema vale para $j-1$, ou seja, $\operatorname{lower}\left(X_{j-1}\right)=$ $\left\lceil\max \left\{\rho_{0}\left(X_{j-1}\right), \rho_{1}\left(X_{j-1}\right)\right\} / 2\right\rceil=j-1$. Vamos provar que lower $\left(X_{j}\right)=j$.

Se $\mathrm{R}_{j}=\mathrm{R}=\left[C_{1}, C_{2}, \cdots, C_{j}\right]$ é uma seqüência retangular de $A$, então, por definição, $\mathrm{R}_{j-1}=\left[C_{1}, C_{2}, \cdots, C_{j-1}\right]$ é uma decomposição seqüencial de $X_{j-1}$. Além disso, se $\rho\left(C_{j}\right)=$ $(a, b)$, então, claramente,

$$
c_{x, y}\left(\mathrm{R}_{j}\right)= \begin{cases}c_{x, y}\left(\mathrm{R}_{j-1}\right)+1 & \text { se }(x, y)=(a, b) \\ c_{x, y}\left(\mathrm{R}_{j-1}\right) & \text { se }(x, y) \neq(a, b) .\end{cases}
$$

Agora, considere $X_{j}=X_{j-1} \oplus C_{j}$. Pela Proposição 4.17, $\rho\left(X_{j}\right)=\rho\left(X_{j-1}\right)+\rho\left(C_{j}\right)$. Temos então que analisar quatro casos possíveis para $C_{j}: \rho_{0}\left(C_{j}\right)+\rho_{1}\left(C_{j}\right)=4,3,2$ ou 1 .

Caso 1: $\rho_{0}\left(C_{j}\right)+\rho_{1}\left(C_{j}\right)=4$

Neste caso, $\rho_{0}\left(C_{j}\right)=\rho_{1}\left(C_{j}\right)=2$. Dessa forma, se $\rho_{0}\left(X_{j-1}\right) \geq \rho_{1}\left(X_{j-1}\right)$, então

a Condição $(a)$ do Lema 5.25 é satisfeita. Caso contrário, a Condição $(c)$ do Le- 
ma 5.25 é satisfeita. Logo, uma vez que uma das condições do Lema 5.25 é satisfeita, lower $\left(X_{j}\right)=\operatorname{lower}\left(X_{j-1}\right)+1=(j-1)+1=j$.

Caso 2: $\rho_{0}\left(C_{j}\right)+\rho_{1}\left(C_{j}\right)=3$

Neste caso, temos duas possibilidades: $\rho\left(C_{j}\right)=(1,2)$ ou $\rho\left(C_{j}\right)=(2,1)$. Provaremos somente a primeira. A prova para $\rho\left(C_{j}\right)=(2,1)$ pode ser feita de uma maneira similar.

Uma vez que $\rho\left(C_{j}\right)=(1,2)$, pela Igualdade $(3), c_{1,2}\left(\mathrm{R}_{j}\right)=c_{1,2}\left(\mathrm{R}_{j-1}\right)+1$; e, para todo $(x, y) \neq(1,2), c_{x, y}\left(\mathrm{R}_{j}\right)=c_{x, y}\left(\mathrm{R}_{j-1}\right)$. Conseqüentemente, uma vez que $c_{1,2}\left(\mathrm{R}_{j}\right)=$ $c_{1,2}\left(\mathrm{R}_{j-1}\right)+1, c_{1,2}\left(\mathrm{R}_{j}\right)>0$, e, pela Restrição $(k)$ da definição de seqüência retangular, $c_{2,1}\left(\mathrm{R}_{j}\right)=0$. Uma vez que $c_{2,1}\left(\mathrm{R}_{j}\right)=c_{2,1}\left(\mathrm{R}_{j-1}\right)$, então $c_{2,1}\left(\mathrm{R}_{j-1}\right)=0$.

Pela ordem escolhida para construir SeqQ, uma vez que $\rho_{0}\left(C_{j}\right)+\rho_{1}\left(C_{j}\right)=3$, temos que $c_{2,0}\left(\mathrm{R}_{j-1}\right)=c_{0,2}\left(\mathrm{R}_{j-1}\right)=c_{1,1}\left(\mathrm{R}_{j-1}\right)=c_{1,0}\left(\mathrm{R}_{j-1}\right)=c_{0,1}\left(\mathrm{R}_{j-1}\right)=0$.

Assim, aplicando as Igualdades (1) e (2) para $X_{j-1}$, temos que

$$
\begin{aligned}
& \rho_{0}\left(X_{j-1}\right)=2 c_{2,2}\left(\mathrm{R}_{j-1}\right)+c_{1,2}\left(\mathrm{R}_{j-1}\right) \mathrm{e} \\
& \rho_{1}\left(X_{j-1}\right)=2\left[c_{2,2}\left(\mathrm{R}_{j-1}\right)+c_{1,2}\left(\mathrm{R}_{j-1}\right)\right] .
\end{aligned}
$$

Logo, $\rho_{1}\left(X_{j-1}\right) \geq \rho_{0}\left(X_{j-1}\right)$. Dessa forma, uma vez que $\rho\left(C_{j}\right)=(1,2)$, então a Condição $(d)$ do Lema 5.25 é satisfeita. Portanto, lower $\left(X_{j}\right)=\operatorname{lower}\left(X_{j-1}\right)+1=$ $(j-1)+1=j$.

Caso 3: $\rho_{0}\left(C_{j}\right)+\rho_{1}\left(C_{j}\right)=2$

Neste caso, temos três possibilidades: (3.a) $\rho\left(C_{j}\right)=(1,1) ;(3 . b) \rho\left(C_{j}\right)=(2,0)$ ou (3.c) $\rho\left(C_{j}\right)=(0,2)$.

(3.a) Suponha que $\rho\left(C_{j}\right)=(1,1)$. Assim, pela Igualdade (3), temos que $c_{1,1}\left(\mathrm{R}_{j}\right)=$ $c_{1,1}\left(\mathrm{R}_{j-1}\right)+1$; e $c_{x, y}\left(\mathrm{R}_{j}\right)=c_{x, y}\left(\mathrm{R}_{j-1}\right)$, para todo $(x, y) \neq(1,1)$. Conseqüentemente, uma vez que $c_{1,1}\left(\mathrm{R}_{j}\right)=c_{1,1}\left(\mathrm{R}_{j-1}\right)+1, c_{1,1}\left(\mathrm{R}_{j}\right)>0$. Dessa forma, pela Restrição $(f)$ da definição de seqüência retangular, temos que $c_{1,1}\left(R_{j}\right)=1$. Logo, uma vez que $c_{1,1}\left(\mathrm{R}_{j}\right)=c_{1,1}\left(\mathrm{R}_{j-1}\right)+1$, temos que $c_{1,1}\left(\mathrm{R}_{j-1}\right)=0$.

Uma vez que $\rho_{0}\left(C_{j}\right)+\rho_{1}\left(C_{j}\right)=2$ e pela ordem escolhida para construir a seqüência SeqQ, temos que $c_{1,0}\left(\mathrm{R}_{j}\right)=c_{0,1}\left(\mathrm{R}_{j}\right)=0$. Assim, pela Igualdade (3), $c_{1,0}\left(\mathrm{R}_{j-1}\right)=c_{0,1}\left(\mathrm{R}_{j-1}\right)=0$.

Como $c_{1,1}\left(R_{j}\right)=1$, então, pelas Restrições $(g)$ e $(i)$ da definição de seqüência retangular, $c_{2,0}\left(R_{j}\right)=c_{0,2}\left(R_{j}\right)=0$. Conseqüentemente, pela Igualdade (3), $c_{2,0}\left(\mathrm{R}_{j-1}\right)=c_{0,2}\left(\mathrm{R}_{j-1}\right)=0$. Portanto, $c_{1,1}\left(\mathrm{R}_{j-1}\right)=c_{1,0}\left(\mathrm{R}_{j-1}\right)=c_{0,1}\left(\mathrm{R}_{j-1}\right)=$ $c_{2,0}\left(\mathrm{R}_{j-1}\right)=c_{0,2}\left(\mathrm{R}_{j-1}\right)=0$. Aplicando as Igualdades (1) e (2) para $X_{j-1}$, temos que

$$
\begin{aligned}
& \rho_{0}\left(X_{j-1}\right)=2\left[c_{2,2}\left(\mathrm{R}_{j-1}\right)+c_{2,1}\left(\mathrm{R}_{j-1}\right)\right]+c_{1,2}\left(\mathrm{R}_{j-1}\right) \mathrm{e} \\
& \rho_{1}\left(X_{j-1}\right)=2\left[c_{2,2}\left(\mathrm{R}_{j-1}\right)+c_{1,2}\left(\mathrm{R}_{j-1}\right)\right]+c_{2,1}\left(\mathrm{R}_{j-1}\right) .
\end{aligned}
$$

Temos dois subcasos para analisar: (3.a.i) $c_{1,2}\left(\mathrm{R}_{j-1}\right)=0$ e $c_{2,1}\left(\mathrm{R}_{j-1}\right)=0$; e (3.a.ii) $c_{1,2}\left(\mathrm{R}_{j-1}\right)>0$ ou $c_{2,1}\left(\mathrm{R}_{j-1}\right)>0$. 
(3.a.i) Se $c_{1,2}\left(\mathrm{R}_{j-1}\right)=0$ e $c_{2,1}\left(\mathrm{R}_{j-1}\right)=0$, então $\rho_{0}\left(X_{j-1}\right)=2 c_{2,2}\left(\mathrm{R}_{j-1}\right) \mathrm{e}$ $\rho_{1}\left(X_{j-1}\right)=2 c_{2,2}\left(\mathrm{R}_{j-1}\right)$. Logo, $\rho_{0}\left(X_{j-1}\right)=\rho_{1}\left(X_{j-1}\right)$ e $\rho_{0}\left(X_{j-1}\right)$ é um número par. Dessa forma, uma vez que $\rho\left(C_{j}\right)=(1,1)$, então a Condição $(b)$ do Lema 5.25 é satisfeita. Portanto, $\operatorname{lower}\left(X_{j}\right)=$ lower $\left(X_{j-1}\right)+1=(j-1)+1=j$.

(3.a.ii) Supunha que $c_{1,2}\left(\mathrm{R}_{j-1}\right)>0$ ou $c_{2,1}\left(\mathrm{R}_{j-1}\right)>0$. Se $c_{1,2}\left(\mathrm{R}_{j-1}\right)>$ 0 , então, pela Restrição $(k)$ da definição de seqüência retangular, $c_{2,1}\left(\mathrm{R}_{j-1}\right)=0$. Assim, $\rho_{0}\left(X_{j-1}\right)=2 c_{2,2}\left(\mathrm{R}_{j-1}\right)+c_{1,2}\left(\mathrm{R}_{j-1}\right)$ e $\rho_{1}\left(X_{j-1}\right)=2\left[c_{2,2}\left(\mathrm{R}_{j-1}\right)+c_{1,2}\left(\mathrm{R}_{j-1}\right)\right]$. Logo, $\rho_{1}\left(X_{j-1}\right) \geq \rho_{0}\left(X_{j-1}\right)$ e $\rho_{1}\left(X_{j-1}\right)$ é um número par. Dessa forma, uma vez que $\rho\left(C_{j}\right)=$ $(1,1)$, então a Condição $(d)$ do Lema 5.25 é satisfeita. Portanto, lower $\left(X_{j}\right)=\operatorname{lower}\left(X_{j-1}\right)+1=(j-1)+1=j$.

Se $c_{2,1}\left(R_{j-1}\right)>0$, pode-se provar, de uma maneira similar ao caso $c_{1,2}\left(\mathrm{R}_{j-1}\right)>0$, que lower $\left(X_{j}\right)=j$.

(3.b) Agora, suponha que $\rho\left(C_{j}\right)=(2,0)$. Assim, pela Igualdade (3), $c_{2,0}\left(\mathrm{R}_{j}\right)=$ $c_{2,0}\left(\mathrm{R}_{j-1}\right)+1$; e $c_{x, y}\left(\mathrm{R}_{j}\right)=c_{x, y}\left(\mathrm{R}_{j-1}\right)$, para todo $(x, y) \neq(2,0)$. Conseqüentemente, uma vez que $c_{2,0}\left(\mathrm{R}_{j}\right)=c_{2,0}\left(\mathrm{R}_{j-1}\right)+1, c_{2,0}\left(\mathrm{R}_{j}\right)>0$. Dessa forma, pela Restrição $(a)$ da definição de seqüência retangular, $c_{0,2}\left(R_{j}\right)=0$ e, pelas Retrições $(g)$ e $(h)$ da definição de seqüência retangular, $c_{1,1}\left(R_{j}\right)=c_{1,2}\left(R_{j}\right)=0$. Assim, pela Igualdade (3), $c_{0,2}\left(\mathrm{R}_{j-1}\right)=c_{1,1}\left(\mathrm{R}_{j-1}\right)=c_{1,2}\left(\mathrm{R}_{j-1}\right)=0$. Desta forma, aplicando as Igualdades (1) e (2) para $X_{j-1}$, temos que

$$
\begin{aligned}
& \rho_{0}\left(X_{j-1}\right)=2\left[c_{2,2}\left(\mathrm{R}_{j-1}\right)+c_{2,1}\left(\mathrm{R}_{j-1}\right)+c_{2,0}\left(\mathrm{R}_{j-1}\right)\right] \mathrm{e} \\
& \rho_{1}\left(X_{j-1}\right)=2 c_{2,2}\left(\mathrm{R}_{j-1}\right)+c_{2,1}\left(\mathrm{R}_{j-1}\right) .
\end{aligned}
$$

Logo, $\rho_{0}\left(X_{j-1}\right) \geq \rho_{1}\left(X_{j-1}\right)$ Assim, uma vez que $\rho\left(C_{j}\right)=(2,0)$, então a Condição $(a)$ do Lema 5.25 é satisfeita. Portanto, lower $\left(X_{j}\right)=\operatorname{lower}\left(X_{j-1}\right)+1=$ $(j-1)+1=j$.

(3.c) A prova para o caso $\rho\left(C_{j-1}\right)=(0,2)$ pode ser feita de uma maneira análoga ao caso $\rho\left(C_{j-1}\right)=(2,0)$.

Caso 4: $\rho_{0}\left(C_{j}\right)+\rho_{1}\left(C_{j}\right)=1$

Neste caso, temos duas possibilidades: $\rho\left(C_{j}\right)=(1,0)$ ou $\rho\left(C_{j}\right)=(0,1)$. Provaremos somente a primeira. A prova para $\rho\left(C_{j}\right)=(0,1)$ pode ser feita de uma maneira similar.

Uma vez que $\rho\left(C_{j}\right)=(1,0)$, pela Igualdade $(3), c_{1,0}\left(\mathrm{R}_{j}\right)=c_{1,0}\left(\mathrm{R}_{j-1}\right)+1$ e, para todo $(x, y) \neq(1,0), c_{x, y}\left(\mathrm{R}_{j}\right)=c_{x, y}\left(\mathrm{R}_{j-1}\right)$. Conseqüentemente, uma vez que $c_{1,0}\left(\mathrm{R}_{j}\right)=c_{1,0}\left(\mathrm{R}_{j-1}\right)+1, c_{1,0}\left(\mathrm{R}_{j}\right)>0$. Assim, pela Restrição $(f)$ da definição de seqüência retangular, $c_{0,1}\left(\mathrm{R}_{j}\right)=c_{1,1}\left(\mathrm{R}_{j}\right)=0$ e $c_{1,0}\left(\mathrm{R}_{j}\right)=1$. Dessa forma, pela Igualdade $(3), c_{1,0}\left(\mathrm{R}_{j-1}\right)=c_{0,1}\left(\mathrm{R}_{j-1}\right)=c_{1,1}\left(\mathrm{R}_{j-1}\right)=0$. Uma vez que, $c_{1,0}\left(\mathrm{R}_{j}\right)>0$, pelas Restrições $(c)$ e $(e)$ da definição de seqüência retangular, $c_{0,2}\left(\mathrm{R}_{j}\right)=c_{1,2}\left(\mathrm{R}_{j}\right)=0$. Assim, pela Igualdade $(3), c_{0,2}\left(R_{j-1}\right)=c_{1,2}\left(R_{j-1}\right)=0$. Portanto, $c_{1,0}\left(R_{j-1}\right)=c_{0,1}\left(R_{j-1}\right)=$ $c_{1,1}\left(\mathrm{R}_{j-1}\right)=c_{0,2}\left(\mathrm{R}_{j-1}\right)=c_{1,2}\left(\mathrm{R}_{j-1}\right)=0$.

Logo, aplicando as Igualdades (1) e (2) para $X_{j-1}$, temos que 


$$
\begin{aligned}
& \rho_{0}\left(X_{j-1}\right)=2\left[c_{2,2}\left(\mathrm{R}_{j-1}\right)+c_{2,1}\left(\mathrm{R}_{j-1}\right)+c_{2,0}\left(\mathrm{R}_{j-1}\right)\right] \mathrm{e} \\
& \rho_{1}\left(X_{j-1}\right)=2 c_{2,2}\left(\mathrm{R}_{j-1}\right)+c_{2,1}\left(\mathrm{R}_{j-1}\right) .
\end{aligned}
$$

Assim, $\rho_{0}\left(X_{j-1}\right) \geq \rho_{1}\left(X_{j-1}\right)$ e $\rho_{0}\left(X_{j-1}\right)$ é um número par. Desta forma, uma vez que $\rho\left(C_{j}\right)=(1,0)$, então a Condição $(b)$ do Lema 5.25 é satisfeita. Portanto, $\operatorname{lower}\left(X_{j}\right)=$ lower $\left(X_{j-1}\right)+1=(j-1)+1=j$. 


\section{Capítulo 6}

\section{Decomposição de Elementos Estruturantes Simplesmente Conexos}

Park e Chin [35] desenvolveram um complexo algoritmo para encontrar uma decomposição ótima de EE's simplesmente conexos (isto é, EE's 8-conexos que não contém buracos) impondo a restrição de que todos elementos nesta decomposição sejam também simplesmente conexos. Neste capítulo, mostraremos que existem famílias infinitas de EE's simplesmente conexos que têm uma decomposição segundo a definição dada no Capítulo 4, mas não são decomponíveis segundo a definição de Park e Chin. Além disso, faremos um comentário sobre a complexidade do algoritmo de Park e Chin, uma vez que uma análise da complexidade de tempo do algoritmo não foi feita no trabalho deles.

\subsection{O Algoritmo de Park e Chin}

Park e Chin [35] apresentaram uma extensão do algoritmo do Xu (que decompõe EE's convexos) para decompor EE's simplesmente conexos (isto é, EE's 8-conexos que não contêm buracos), impondo a restrição de que todos elementos na decomposição sejam também simplesmente conexos.

O algoritmo deles é bastante complexo e pode ser basicamente dividido em três passos. Seja $A$ um EE simplesmente conexo. Então, os passos do algoritmo de Park e Chin são dados a seguir.

Passo 1: $\quad$ O algoritmo verifica se $A$ satisfaz algumas condições necessárias para decomposição. Se estas condições não são satisfeitas, então $A$ não tem decomposição e o algoritmo pára.

Passo 2: O algoritmo monta um sistema linear de inequações com um número fixo de variáves e inicia uma busca por uma solução inteira para este sistema. 


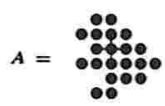

(a)

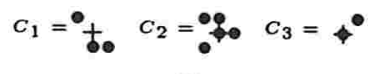

(b)

Figura 6.1: (a) Um EE $A$ (b) Uma decomposição de $A$.

Se nenhuma solução inteira é encontrada, então $A$ não tem decomposição e o algoritmo pára. Caso contrário, o algoritmo obtém uma solução do sistema e vai para o passo seguinte.

Passo 3: A partir da solução encontrada no passo anterior, o algoritmo procura uma decomposição ótima de $A$ usando uma extensão do intricado processo de otimização (Passo 2) do algoritmo do Xu [49].

O ponto crucial da complexidade do algoritmo de Park e Chin está no Passo 2. No trabalho deles, a maneira como o algoritmo busca por uma solução inteira do sistema linear não é mencionada. Sabemos que, teoricamente, existe um algoritmo polinomial para encontrar soluções inteiras de sistemas de inequações lineares com um número fixo de variáveis [40, p. 256]. No entanto, no trabalho deles, Park e Chin [35] não analisaram a complexidade de tempo do algoritmo deles.

Estudando o problema da decomposição de EE's, observamos que existem alguns EE's simplesmente conexos, que não são decomponíveis segundo a definição dada por Park e Chin, mas que podem ter uma decomposição segundo a definição dada no Capítulo 4, ou seja, uma decomposição como uma seqüência de subconjuntos do quadrado elementar (sem a restrição que estes subconjuntos sejam simplesmente conexos).

Por exemplo, na Figura 6.1.a, apresentamos um EE simplesmente conexo $A$ que não é decomponível segundo a definição de Park e Chin [35, Exemplo 3, p. 10]. Mas, se retirarmos a imposição de que os subconjuntos na decomposição sejam simplesmente conexos, então $A$ tem uma decomposição que está apresentada na Figura 6.1.b. Note que o primeiro subconjunto nesta decomposição não é simplemente conexo.

Neste capítulo, mostraremos que existem famílias infinitas de EE's simplesmente conexos que têm decomposição, mas que não são decomponíveis segundo a definição de Park e Chin. Daremos também alguns comentários sobre a complexidade do algoritmo deles.

Seguindo esta breve introdução, a Seção 6.2 apresenta algumas famílias infinitas de EE's e prova que os EE's pertencentes a essas famílias são simplesmente conexos e que não têm decomposição segundo a definição de Park and Chin. A Seção 6.3 fornece alguns comentários sobre a complexidade de tempo do algoritmo de Park e Chin. Finalmente, na Seção 6.4 apresentamos algumas conclusões deste capítulo. 


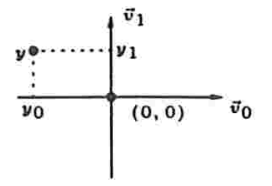

Figura 6.2: Os eixos Cartesianos $\vec{v}_{0}$ e $\vec{v}_{1}$.

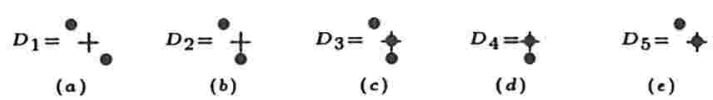

Figura 6.4: $(a)-(e)$ Os EE's $D_{1}, D_{2}, D_{3}, D_{4}$ e $D_{5}$.

\subsection{Famílias Infinitas de Elementos Estruturantes}

Neste capítulo, consideraremos dois eixos Cartesianos $\vec{v}_{0}$ e $\vec{v}_{1}$ que intersectam a origem de $\mathbb{Z}^{2}$ e cujas inclinações são, respectivamente, 0 e 90 graus (veja a Figura 6.2). Para um ponto $x \in \mathbb{Z}^{2}$, denotaremos $x_{0}$ e $x_{1}$ as projeções ortogonais de $x$ nos eixos Cartesianos $\vec{v}_{0}$ e $\vec{v}_{1}$, respectivamente. Por exemplo, as projeções ortogonais do ponto $y=(-5,2) \in \mathbb{Z}^{2}$ são $y_{0}=-5$ e $y_{1}=2$ (veja a Figura 6.2 ). Note que $\vec{v}_{0}=\vec{u}_{2}$ e $\vec{v}_{1}=-\vec{u}_{0}$, onde $\vec{u}_{0}$ e $\vec{u}_{2}$ são os eixos Cartesianos definidos no Capítulo 4 (veja estas definições na página 47).

Seja $A$ um EE. Para $i=0,1$, sejam $\operatorname{MAV}_{i}(A)$ e $\operatorname{MIV}_{i}(A)$, respectivamente, as projeções ortogonais máxima e mínima sobre os eixos Cartesianos $\vec{v}_{i}$ dentre todos pontos de $A$, isto é, $\operatorname{MAV}_{i}(A)=\max \left\{x_{i}: x \in A\right\}$ e $\operatorname{MIV}_{i}(A)=\min \left\{x_{i}: x \in A\right\}$. Por exemplo, as projeções ortogonais máxima e mínima do EE $A$ apresentado na Figura 6.3 são, $\operatorname{MAV}_{0}(A)=3$, $\operatorname{MAV}_{1}(A)=1, \operatorname{MIV}_{0}(A)=-1$ e $\operatorname{MIV}_{1}(A)=-2$.

Note que $\operatorname{MAV}_{0}(A)=\operatorname{MAX}_{2}(A), \operatorname{MIV}_{0}(A)=\operatorname{MIN}_{2}(A), \operatorname{MAV}_{1}(A)=-\operatorname{MAX}_{0}(A) \mathrm{e}$ $\operatorname{MIV}_{1}(A)=-\operatorname{MIN}_{0}(A)$, onde $\operatorname{MAX}_{2}(A), \operatorname{MIN}_{2}(A), \operatorname{MAX}_{0}(A)$ e $\operatorname{MIN}_{0}(A)$ são os valores definidos no Capítulo 4 (veja estas definições na página 47 ).

A seguir apresentamos duas propriedades de $\operatorname{MAV}_{i}(A)$ e $\operatorname{MIV}_{i}(A)$, dadas pelos Lemas 6.1 e 6.2, que são necessárias para a construção das famílias de EE's simplesmente conexos. O Lema 6.1 (respectivamente, Lema 6.2) é uma conseqüência imediata do Lema 4.10 (respectivamente, Lema 4.11).

Lema 6.1 Se $X$ e $Y$ são EE's, então $\operatorname{MAV}_{i}(X \oplus Y)=\operatorname{MAV}_{i}(X)+\operatorname{MAV}_{i}(Y)$ e $\operatorname{MIV}_{i}(X \oplus$ $Y)=\operatorname{MIV}_{i}(X)+\operatorname{MIV}_{i}(Y)$.

Lema 6.2 Se $X$ é um EE e $h \in \mathbb{Z}^{2}$, então, para todo $i \in\{0,1\}, \operatorname{MAV}_{i}\left(X_{h}\right)=\operatorname{MAV}_{i}(X)+$ $h_{i} e \operatorname{MIV}_{i}\left(X_{h}\right)=\operatorname{MIV}_{i}(X)+h_{i}$.

Para construir as famílias infinitas de EE's simplesmente conexos, considere os seguin- 


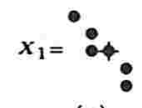

(a)

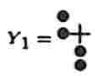

(b)

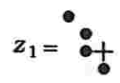

(c)

Figura 6.5: $(a)-(c)$ EE's $X_{1}, Y_{1}$ e $Z_{1}$.

tes EE's: $D_{1}=\left\{d_{1}, d_{2}\right\}, D_{2}=\left\{d_{1}, d_{4}\right\}, D_{3}=\left\{d_{1}, d_{3}, d_{4}\right\}, D_{4}=\left\{d_{3}, d_{4}\right\}$ e $D_{5}=\left\{d_{1}, d_{3}\right\}$, onde $d_{1}=(-1,1), d_{2}=(1,-1), d_{3}=(0,0)$ e $d_{4}=(0,-1)$. Estes EE's estão apresentados na Figura 6.4. Em particular, observe que $\operatorname{MAV}_{0}\left(D_{3}\right)=\operatorname{MAV}_{0}\left(D_{4}\right)=\operatorname{MIV}_{0}\left(D_{4}\right)=$ $\operatorname{MAV}_{0}\left(D_{5}\right)=0$ e $\operatorname{MIV}_{0}\left(D_{3}\right)=\operatorname{MIV}_{0}\left(D_{5}\right)=-1$

Agora, para todo inteiro $i>0$, considere os EE's $X_{i}=i D_{1} \oplus D_{3}, Y_{i}=i D_{2} \oplus D_{4} \mathrm{e}$ $Z_{i}=i D_{2} \oplus D_{5}$. Por exemplo, os EE's $X_{1}, Y_{1}$ e $Z_{1}$ estão apresentados na Figura 6.5 e os EE's $X_{6}, Y_{7}$ e $Z_{7}$ podem ser vistos na Figura 6.9.

Mostraremos que as famílias $\mathcal{X}=\left\{X_{i}: i>0\right\}, \mathcal{Y}=\left\{Y_{i}: i>0\right\}$ e $\mathcal{Z}=\left\{Z_{i}: i>0\right\}$ são famílias de EE's simplesmente conexos que têm uma decomposição, mas não são decomponiveis segundo a definição de Park e Chin.

Dividimos esta seção em duas partes. Na primeira, mostraremos que cada EE em $\mathcal{X}, \mathcal{Y}$ e $\mathcal{Z}$ são simplesmente conexos. Na segunda parte, provaremos que qualquer decomposição de $X_{i}, Y_{i}$ e $Z_{i}$ contém pelo menos um elemento que não é simplesmente conexo.

\subsubsection{Famílias de Elementos Estruturantes Simplesmente Cone- xos}

Para provar que os elementos das famílias $\mathcal{X}, \mathcal{Y}$ e $\mathcal{Z}$ são simplesmente conexos, precisamos do seguinte resultado.

Proposição 6.3 Sejam $X$ e $Y$ dois EE's simplesmente conexos. tais que os pontos $x=$ $\left(\operatorname{MAV}_{0}(X), \operatorname{MIV}_{1}(X)\right)$ e $y=\left(\operatorname{MIV}_{0}(Y), \operatorname{MAV}_{1}(Y)\right)$ estão em $X$ e $Y$, respectivamente. Se $0 \leq \operatorname{MIV}_{0}(Y)-\operatorname{MAV}_{0}(X) \leq 1$ e $0 \leq \operatorname{MIV}_{1}(X)-\operatorname{MAV}_{1}(Y) \leq 1$, então $X \cup Y$ é também um EE simplesmente conexo.

Prova: Podemos ver facilmente que se $X$ e $Y$ satisfazem as restrições $0 \leq \operatorname{MIV}_{0}(Y)-$ $\operatorname{MAV}_{0}(X) \leq 1$ e $0 \leq \operatorname{MIV}_{1}(X)-\operatorname{MAV}_{1}(Y) \leq 1$, então temos somente quatro possibilidades para $X$ e $Y$, como mostrado na Figura 6.6. Uma vez que $X$ e $Y$ são EE's simplesmente conexos e $x \in X$ e $y \in Y$, então claramente $X \cup Y$ é também um EE simplesmente conexo.

Note que, para todo inteiro $i>0, i D_{1}=\left\{h_{0}, h_{1}, \ldots, h_{i}\right\}$ e $i D_{2}=\left\{g_{0}, g_{1}, \ldots, g_{i}\right\}$, onde $h_{0}=g_{0}=(-i, i)$ e, para todo $j \in\{1,2, \ldots, i\}, h_{j}=h_{j-1}+(2,-2)$ e $g_{j}=g_{j-1}+(1,-2)$. Por exemplo, a Figura 6.7 mostra os EE's $i D_{1}$ e $i D_{2}$. Observando esta figura, podemos ver facilmente que $\operatorname{MAV}_{0}\left(i D_{1}\right)=i, \operatorname{MIV}_{1}\left(i D_{1}\right)=-i, \operatorname{MAV}_{0}\left(i D_{2}\right)=0$ e $\operatorname{MIV}_{1}\left(i D_{2}\right)=-i$. 


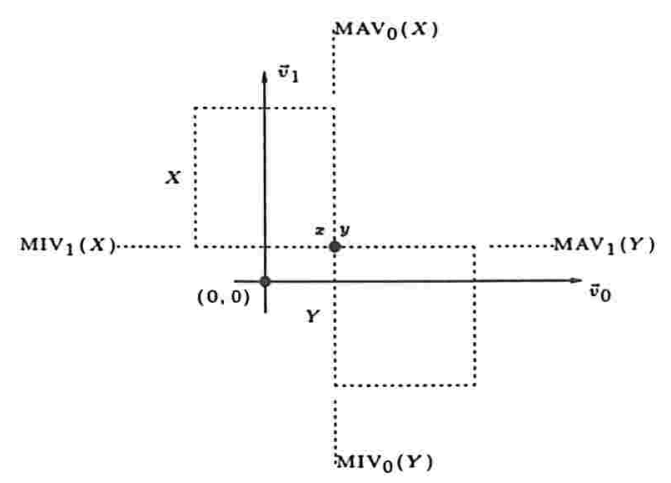

(a)

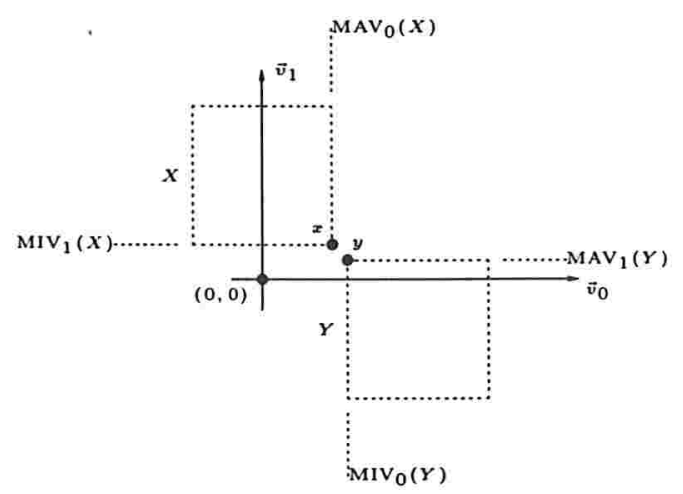

(c)

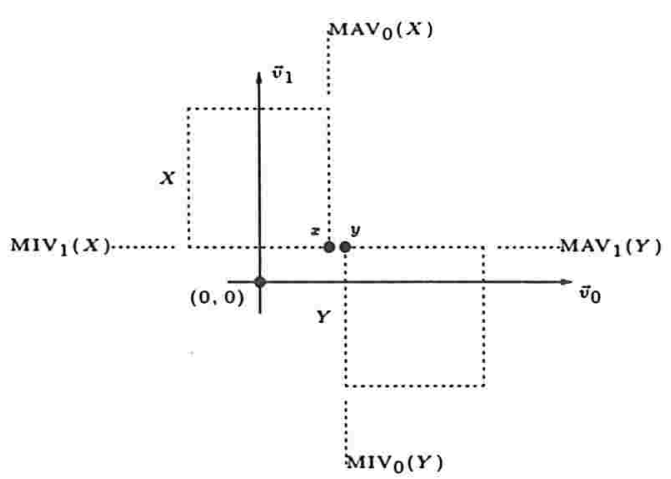

(b)

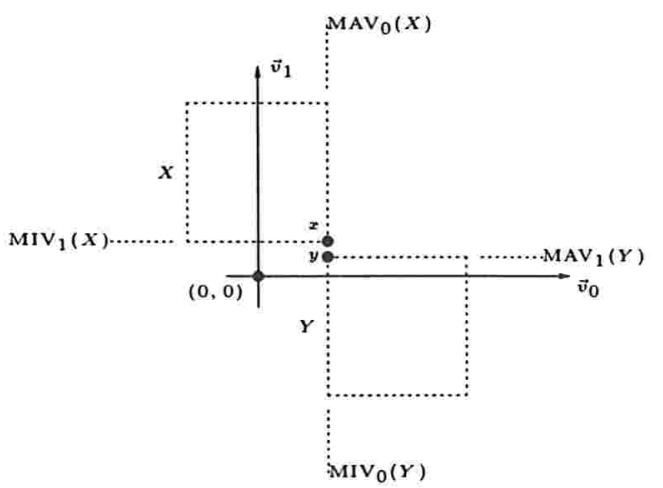

(d)

Figura 6.6: $(a)-(d)$ As quatro possibilidades para $X$ e $Y$.

Além disso, para todo $i>0$, $i D_{1}$ e $i D_{2}$ não são EE's simplesmente conexos. Observe também que $d_{2}-d_{1}=(2,-2)$ e $d_{4}-d_{1}=(1,-2)$. Assim, podemos escrever as expressões para $h_{j}$ e $g_{j}$ como $h_{j}+d_{1}=h_{j-1}+d_{2}$ e $g_{j}+d_{1}=g_{j-1}+d_{4}$.

A Proposição 6.4 fornece uma propriedade dos EE's $X_{i}, Y_{i}$ e $Z_{i}$. O Teorema 6.5 prova que os EE's $X_{i}, Y_{i}$ e $Z_{i}$ são simplesmente conexos.

Proposição 6.4 Para todo inteiro $i>0$,

$$
\begin{array}{ll}
\text { (a) } & X_{i+1}=\left(X_{i}\right)_{d_{1}} \cup\left(D_{3}\right)_{p_{i}}, \\
\text { (b) } Y_{i+1}=\left(Y_{i}\right)_{d_{1}} \cup\left(D_{4}\right)_{q_{i}}, \\
\text { (c) } Z_{i+1}=\left(Z_{i}\right)_{d_{1}} \cup\left(D_{5}\right)_{q_{i}},
\end{array}
$$

onde, $p_{i}=(i+1,-i-1)$ e $q_{i}=(0,-i-1)$.

Prova: Provaremos somente a primeira igualdade. As outras podem ser provadas de uma maneira análoga. Dividiremos esta prova em duas partes. Na primeira parte, pro- 


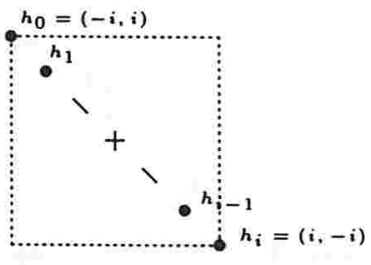

(a)

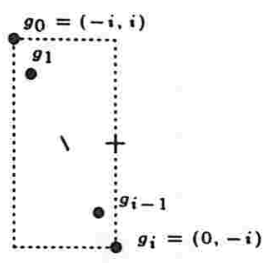

(b)

Figura 6.7: $(a)-(b)$ EE's $i D_{1}$ e $i D_{2}$.

varemos que $(i+1) D_{1}=i D_{1} \oplus D_{1}=\left(i D_{1}\right)_{d_{1}} \cup\left\{p_{i}\right\}$ e, na segunda, mostraremos que $X_{i+1}=\left(X_{i}\right)_{d_{1}} \cup\left(D_{3}\right)_{p_{i}}$.

$$
\begin{aligned}
i D_{1} \oplus D_{1} & =\left\{h_{0}, h_{1}, \cdots, h_{i-1}, h_{i}\right\} \oplus\left\{d_{1}, d_{2}\right\} \\
& =\left\{h_{0}+d_{1}, \cdots, h_{i-1}+d_{1}, h_{i}+d_{1}\right\} \cup\left\{h_{0}+d_{2}, \cdots, h_{i-1}+d_{2}, h_{i}+d_{2}\right\} \\
& \quad \text { (pela definição da adição de Minkowski) } \\
& \left\{h_{0}+d_{1}, \cdots, h_{i}+d_{1}, h_{i}+d_{2}\right\} \\
& =\left\{h_{0}, h_{1}, \cdots, h_{i}\right\}_{d_{1}} \cup\left\{h_{i}+d_{2}\right\} \\
& \left.=\left(i D_{1}\right)_{d_{1}} \cup\left\{p_{i}\right\} \quad \text { (uma vez que } h_{j}+d_{1}=h_{j-1}+d_{2}, \text { para todo } j>0\right)
\end{aligned}
$$

Agora, o conjunto $X_{i+1}$.

$$
\begin{aligned}
X_{i+1} & =(i+1) D_{1} \oplus D_{3} \\
& =\left(i D_{1} \oplus D_{1}\right) \oplus D_{3} \\
& =\left(\left(i D_{1}\right)_{d_{1}} \cup\left\{p_{i}\right\}\right) \oplus D_{3} \\
& =\left(\left(i D_{1}\right)_{d_{1}} \oplus D_{3}\right) \cup\left(\left\{p_{i}\right\} \oplus D_{3}\right) \\
& =\left(\left(i D_{1}\right)_{d_{1}} \oplus D_{3}\right) \cup\left(D_{3}\right)_{p_{i}} \\
& =\left(i D_{1} \oplus D_{3}\right)_{d_{1}} \cup\left(D_{3}\right)_{p_{i}} \text { (pela P } \\
& \\
& =\left(X_{i}\right)_{d_{1}} \cup\left(D_{3}\right)_{p_{i}}
\end{aligned}
$$

Na Figura 6.8, mostramos os EE's $X_{i+1}=\left(X_{i}\right)_{d_{1}} \cup\left(D_{3}\right)_{p_{i}}, Y_{i+1}=\left(Y_{i}\right)_{d_{1}} \cup\left(D_{4}\right)_{q_{i}}$ e $Z_{i+1}=\left(Z_{i}\right)_{d_{1}} \cup\left(D_{5}\right)_{q_{i}}$.

Agora, estamos prontos para provar que, para $i>0$, os EE's $X_{i}, Y_{i}$ e $Z_{i}$ são simplesmente conexos.

Teorema 6.5 Para todo inteiro $i>0, X_{i}, Y_{i}$ e $Z_{i}$ são EE's simplesmente conexos.

Prova: Provaremos que para todo inteiro $i>0, X_{i}$ é simplesmente conexo. De uma maneira análoga, pode-se provar que $Y_{i}$ e $Z_{i}$ são também EE's simplesmente conexos. 


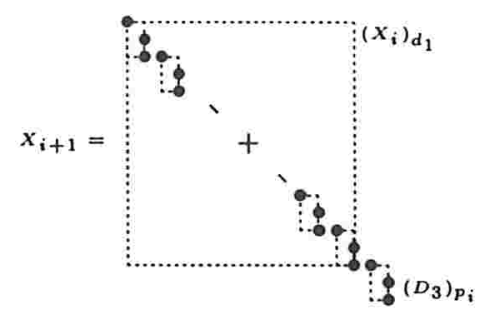

(a)

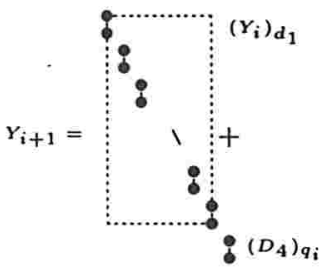

(b)

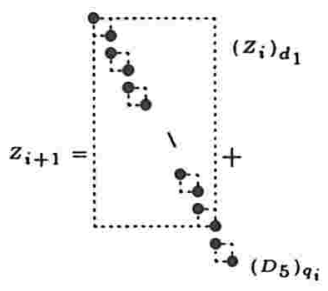

(c)

Figura 6.8: $(a) X_{i+1}=\left(X_{i}\right)_{d_{1}} \cup\left(D_{3}\right)_{p_{i}}(b) Y_{i+1}=\left(Y_{i}\right)_{d_{1}} \cup\left(D_{4}\right)_{q_{i}}$ e $(c) Z_{i+1}=\left(Z_{i}\right)_{d_{1}} \cup\left(D_{5}\right)_{q_{i}}$.

A prova será por indução em $i$. Se $i=1, X_{1}$ é obviamente simplesmente conexo (veja a Figura 6.5). Agora, suponha por hipótese de indução que, para todo $i>1, X_{i}$ é simplesmente conexo. Mostraremos que $X_{i+1}$ é também simplesmente conexo.

Pela Proposição $6.4, X_{i+1}=\left(X_{i}\right)_{d_{1}} \cup\left(D_{3}\right)_{p_{i}}$. Os EE's $\left(D_{3}\right)_{p_{i}}$ (veja a Figura 6.4) e $\left(X_{i}\right)_{d_{1}}$ (por hipótese de indução) são simplesmente conexos. Observando a Figura 6.8, podemos ver que $0 \leq \operatorname{MIV}_{0}\left(\left(D_{3}\right)_{p_{i}}\right)-\operatorname{MAV}_{0}\left(\left(X_{i}\right)_{d_{1}}\right) \leq 1$ e $0 \leq \operatorname{MIV}_{1}\left(\left(X_{i}\right)_{d_{1}}\right)-\operatorname{MAV}_{1}\left(\left(D_{3}\right)_{p_{i}}\right) \leq 1 \mathrm{e}$ os pontos $x=\left(\operatorname{MAV}_{0}\left(\left(X_{i}\right)_{d_{1}}\right), \operatorname{MIV}_{1}\left(\left(X_{i}\right)_{d_{1}}\right)\right)$ e $y=\left(\operatorname{MIV}_{0}\left(\left(D_{3}\right)_{p_{i}}\right), \operatorname{MAV}_{1}\left(\left(D_{3}\right)_{p_{i}}\right)\right)$ estão em $\left(X_{i}\right)_{d_{1}}$ e $\left(D_{3}\right)_{p_{i}}$, respectivamente. Assim, pela Proposição 6.3, $X_{i+1}=\left(X_{i}\right)_{d_{1}} \cup\left(D_{3}\right)_{p_{i}}$ é um EE simplesmente conexo.

\subsubsection{Um Elemento em qualquer Decomposição não é Simples- mente Conexo}

Para mostrar que um elemento em qualquer decomposição de $X_{i}, Y_{i}$ e $Z_{i}$ não é simplesmente conexo, precisamos antes estudar algumas propriedades desses EE's. Note que, por definição de $X_{i}, Y_{i}$ e $Z_{i}$, podemos escrever as expressões para $X_{i+1}, Y_{i+1}$ e $Z_{i+1}$ em termos da união de duas translações de $X_{i}, Y_{i}$ e $Z_{i}$, respectivamente. Mais formalmente,

(a) $X_{i+1}=\left(i D_{1} \oplus D_{3}\right) \oplus D_{1}=X_{i} \oplus D_{1}=X_{i} \oplus\left\{d_{1}, d_{2}\right\}=\left(X_{i}\right)_{d_{1}} \cup\left(X_{i}\right)_{d_{2}}$;

(b) $Y_{i+1}=\left(i D_{2} \oplus D_{4}\right) \oplus D_{2}=Y_{i} \oplus D_{2}=Y_{i} \oplus\left\{d_{1}, d_{4}\right\}=\left(Y_{i}\right)_{d_{1}} \cup\left(Y_{i}\right)_{d_{4}}$;

(c) $Z_{i+1}=\left(i D_{2} \oplus D_{5}\right) \oplus D_{2}=Z_{i} \oplus D_{2}=Z_{i} \oplus\left\{d_{1}, d_{4}\right\}=\left(Z_{i}\right)_{d_{1}} \cup\left(Z_{i}\right)_{d_{4}}$.

A próxima proposição fornece uma propriedade interessante de $\left(X_{i}\right)_{d_{1}}$ e $\left(X_{i}\right)_{d_{2}} ;\left(Y_{i}\right)_{d_{1}}$ 
$\mathrm{e}\left(Y_{i}\right)_{d_{4}} ;\left(Z_{i}\right)_{d_{1}} \mathrm{e}\left(Z_{i}\right)_{d_{4}}$.

Proposição 6.6 Para todo $i>0$,

(a) $\left(X_{i}\right)_{d_{1}}-\left(X_{i}\right)_{d_{2}} \subseteq\left(D_{3}\right)_{-p_{i}} \quad$ e $\left(X_{i}\right)_{d_{2}}-\left(X_{i}\right)_{d_{1}} \subseteq\left(D_{3}\right)_{p_{i}}$,

(b) $\left(Y_{i}\right)_{d_{1}}-\left(Y_{i}\right)_{d_{4}} \subseteq\left(D_{4}\right)_{-p_{i}} \quad$ e $\left(Y_{i}\right)_{d_{4}}-\left(Y_{i}\right)_{d_{1}} \subseteq\left(D_{4}\right)_{q_{i}}$,

(c) $\left(Z_{i}\right)_{d_{1}}-\left(Z_{i}\right)_{d_{4}} \subseteq\left(D_{4}\right)_{-p_{i}} \quad e \quad\left(Z_{i}\right)_{d_{4}}-\left(Z_{i}\right)_{d_{1}} \subseteq\left(D_{4}\right)_{q_{i}}$,

onde $p_{i}=(i+1,-i-1)$ e $q_{i}=(0,-i-1)$.

Prova: Provaremos somente as inclusões dadas em (a). As outras podem ser provadas de uma maneira similar.

Observe que podemos escrever as expressões de $\left(X_{i}\right)_{d_{1}}$ e $\left(X_{i}\right)_{d_{2}}$ como

$$
\begin{aligned}
\left(X_{i}\right)_{d_{2}}= & \left(i D_{1} \oplus D_{3}\right)_{d_{2}} \\
= & \left(i D_{1}\right)_{d_{2}} \oplus D_{3} \\
& \quad \text { (pela Proposição 3.8.c) } \\
& \left\{h_{0}+d_{2}, h_{1}+d_{2}, \cdots, h_{i-1}+d_{2}, h_{i}+d_{2}\right\} \oplus D_{3} \\
= & \left\{h_{1}+d_{1}, h_{2}+d_{1}, \cdots, h_{i}+d_{1}, h_{i}+d_{2}\right\} \oplus D_{3} \\
\left.\quad \text { (uma vez que } h_{j}+d_{1}=h_{j-1}+d_{2}, \text { para todo } j>0\right) & \\
= & \left(\left\{h_{i}+d_{2}\right\} \cup\left\{h_{1}+d_{1}, h_{2}+d_{1}, \cdots, h_{i}+d_{1}\right\}\right) \oplus D_{3} \\
= & \left(D_{3}\right)_{h_{i}+d_{2}} \cup R_{i}, \text { onde } R_{i}=\left\{h_{1}+d_{1}, h_{2}+d_{1}, \cdots, h_{i}+d_{1}\right\} \oplus D_{3},
\end{aligned}
$$

$\mathrm{e}$

$$
\begin{aligned}
\left(X_{i}\right)_{d_{1}} & =\left(i D_{1} \oplus D_{3}\right)_{d_{1}} \\
& =\left(i D_{1}\right)_{d_{1}} \oplus D_{3} \\
& =\left\{h_{0}+d_{1}, h_{1}+d_{1}, \cdots, h_{i-1}+d_{1}, h_{i}+d_{1}\right\} \oplus D_{3} \\
& =\left(\left\{h_{0}+d_{1}\right\} \cup\left\{h_{1}+d_{1}, h_{2}+d_{1}, \cdots, h_{i}+d_{1}\right\}\right) \oplus D_{3} \\
& =\left(D_{3}\right)_{h_{0}+d_{1}} \cup R_{i} .
\end{aligned}
$$

Assim, $\left(X_{i}\right)_{d_{1}}-\left(X_{i}\right)_{d_{2}} \subseteq\left(D_{3}\right)_{h_{0}+d_{1}}$ e $\left(X_{i}\right)_{d_{2}}-\left(X_{i}\right)_{d_{1}} \subseteq\left(D_{3}\right)_{h_{i}+d_{2}}$. Uma vez que $h_{0}+d_{1}=(-i, i)+(-1,1)=(-i-1, i+1)=-p_{i}$ e $h_{i}+d_{2}=(i,-i)+(1,-1)=$ $(i+1,-i-1)=p_{i}$, então $\left(X_{i}\right)_{d_{1}}-\left(X_{i}\right)_{d_{2}} \subseteq\left(D_{3}\right)_{-p_{i}}$ e $\left(X_{i}\right)_{d_{2}}-\left(X_{i}\right)_{d_{1}} \subseteq\left(D_{3}\right)_{p_{i}}$.

Como conseqüência do último resultado, a próxima proposição fornece uma caracterização se um EE $D$, com $\rho(D) \leq(2,2)$, é um subconjunto de $X_{i+1}, Y_{i+1}$ e $Z_{i+1}$.

Proposição 6.7 Seja $D$ um EE tal que $\rho(D) \leq(2,2)$. Para todo $i>0$,

(a) $D \subseteq X_{i+1}$ se, e somente se, $D \subseteq\left(X_{i}\right)_{d_{1}}$ ou $D \subseteq\left(X_{i}\right)_{d_{2}}$;

(b) $D \subseteq Y_{i+1}$ se, e somente se, $D \subseteq\left(Y_{i}\right)_{d_{1}}$ ou $D \subseteq\left(Y_{i}\right)_{d_{4}}$;

(c) $D \subseteq Z_{i+1}$ se, e somente se, $D \subseteq\left(Z_{i}\right)_{d_{1}}$ ou $D \subseteq\left(Z_{i}\right)_{d_{4}}$.

Prova: Provaremos somente a primeira caracterização. As outras podem ser provadas de maneira análoga. 
$(\Leftarrow)$ Evidentemente, se $D \subseteq\left(X_{i}\right)_{d_{1}}$ ou $D \subseteq\left(X_{i}\right)_{d_{2}}$, então $D \subseteq X_{i+1}=\left(X_{i}\right)_{d_{1}} \cup\left(X_{i}\right)_{d_{2}}$.

$(\Rightarrow)$ Provaremos que se $D \subseteq X_{i+1}$, então $D \subseteq\left(X_{i}\right)_{d_{1}}$ ou $D \subseteq\left(X_{i}\right)_{d_{2}}$.

Suponha por absurdo que $D \nsubseteq\left(X_{i}\right)_{d_{1}}$ e $D \nsubseteq\left(X_{i}\right)_{d_{2}}$. Assim, uma vez que $D \subseteq$ $X_{i+1}=\left(X_{i}\right)_{d_{1}} \cup\left(X_{i}\right)_{d_{2}}$ então existem dois pontos $x, y \in D$ tais que $x \in\left(X_{i}\right)_{d_{1}}-\left(X_{i}\right)_{d_{2}}$ e $y \in\left(X_{i}\right)_{d_{2}}-\left(X_{i}\right)_{d_{1}}$.

Uma vez que, pela Proposição 6.6, $\left(X_{i}\right)_{d_{1}}-\left(X_{i}\right)_{d_{2}} \subseteq\left(D_{3}\right)_{-p_{i}}$ e $\left(X_{i}\right)_{d_{2}}-\left(X_{i}\right)_{d_{1}} \subseteq\left(D_{3}\right)_{p_{i}}$, onde $p_{i}=(i+1,-i-1)$, então $x_{0} \leq \operatorname{MAV}_{0}\left(\left(D_{3}\right)_{-p_{i}}\right)=-i-1$ e $y_{0} \geq \operatorname{MIV}_{0}\left(\left(D_{3}\right)_{p_{i}}\right)=i+1$. Dessa forma, $y_{0}-x_{0} \geq 2 i+2$. Uma vez que $i>0$, então $y_{0}-x_{0}>2$. Conseqüentemente, temos que $\rho_{0}(\{x, y\})>2$. Como $\{x, y\} \subseteq D$, então, claramente, $2<\rho_{0}(\{x, y\}) \leq \rho_{0}(D)$. Mas, isto é uma contradição, uma vez que, por hipótese, $\rho_{0}(D) \leq 2$. Portanto, $D \subseteq\left(X_{i}\right)_{d_{1}}$ ou $D \subseteq\left(X_{i}\right)_{d_{2}}$.

A próxima proposição fornece uma propriedade interessante sobre subconjuntos do quadrado elementar que são invariantes de $X_{i+1}, Y_{i+1}$ e $Z_{i+1}$.

Proposição 6.8 Seja $D$ um subconjunto do quadrado elementar. Para todo $i>0$,

(a) $D$ é um invariante de $X_{i}$ se, e somente se, $D$ é um invariante de $X_{i+1}$;

(b) $D$ é um invariante de $Y_{i}$ se, e somente se, $D$ é um invariante de $Y_{i+1}$;

(c) $D$ é um invariante de $Z_{i}$ se, e somente se, $D$ é um invariante de $Z_{i+1}$.

Prova: Provaremos somente a primeira afirmação. As outras podem ser provadas de maneira similar.

$(\Rightarrow)$ Se $D$ é um invariante de $X_{i}$, então, Pela Proposição $3.17, D$ é também um invariante de $\left(X_{i}\right)_{h}$, para todo ponto $h \in \mathbb{Z}^{2}$. Assim,

$$
\begin{aligned}
X_{i+1}= & \left(X_{i}\right)_{d_{1}} \cup\left(X_{i}\right)_{d_{2}} \\
= & \left(\left(\left(X_{i}\right)_{d_{1}} \ominus D\right) \oplus D\right) \cup\left(\left(\left(X_{i}\right)_{d_{2}} \ominus D\right) \oplus D\right) \\
\quad & \left.\quad \text { uma vez que, para todo } h \in \mathbb{Z}^{2}, D \text { é invariante de }\left(X_{i}\right)_{h}\right) \\
= & \left(\cup\left\{D_{h}: h \in \mathbb{Z}^{2} \text { e } D_{h} \subseteq\left(X_{i}\right)_{d_{1}}\right\}\right) \cup\left(\cup\left\{D_{h}: h \in \mathbb{Z}^{2} \text { e } D_{h} \subseteq\left(X_{i}\right)_{d_{2}}\right\}\right) \\
\quad & \quad \text { (pela Proposição 3.12) } \\
= & \cup\left\{D_{h}: h \in \mathbb{Z}^{2} \text { e }\left(D_{h} \subseteq\left(X_{i}\right)_{d_{1}} \text { ou } D_{h} \subseteq\left(X_{i}\right)_{d_{2}}\right)\right\} \\
= & \cup\left\{D_{h}: h \in \mathbb{Z}^{2} \text { e } D_{h} \subseteq X_{i+1}\right\} \\
= & \left.\left(X_{i+1} \ominus D\right) \oplus D \text { (pela Proposição 6.7, uma vez que } \rho\left(D_{h}\right) \leq(2,2)\right)
\end{aligned}
$$

(pela Proposição 3.12).

Portanto $D$ é um invariante de $X_{i+1}$.

$(\Leftarrow)$ Agora, suponha por absurdo que $D$ não seja um invariante de $X_{i}$. Assim, pela Proposição $3.17, D$ não é um invariante de $\left(X_{i}\right)_{h}$, para todo $h \in \mathbb{Z}^{2}$. Como, pela Proposição 3.13, $\left(\left(X_{i}\right)_{h} \ominus Y\right) \oplus Y \subseteq\left(X_{i}\right)_{h}$, então, $\left(\left(X_{i}\right)_{h} \ominus Y\right) \oplus Y$ é um subconjunto próprio de $\left(X_{i}\right)_{h}$, isto é, para todo $h \in \mathbb{Z}^{2},\left(\left(X_{i}\right)_{h} \ominus Y\right) \oplus Y \subset\left(X_{i}\right)_{h}$. Logo, 


$$
\begin{aligned}
& \left(X_{i+1} \ominus D\right) \oplus D=\cup\left\{D_{h}: h \in \mathbb{Z}^{2} \text { e } D_{h} \subseteq X_{i+1}\right\} \\
& =\cup\left\{D_{h}: h \in \mathbb{Z}^{2} \text { e }\left(D_{h} \subseteq\left(X_{i}\right)_{d_{1}} \text { ou } D_{h} \subseteq\left(X_{i}\right)_{d_{2}}\right)\right\} \\
& \text { (pela Proposição 6.7, uma vez que } \rho\left(D_{h}\right) \leq(2,2) \text { ) } \\
& =\left(\cup\left\{D_{h}: h \in \mathbb{Z}^{2} \text { e } D_{h} \subseteq\left(X_{i}\right)_{d_{1}}\right\}\right) \cup \\
& \left(\cup\left\{D_{h}: h \in \mathbb{Z}^{2} \text { e } D_{h} \subseteq\left(X_{i}\right)_{d_{2}}\right\}\right) \\
& =\left(\left(\left(X_{i}\right)_{d_{1}} \ominus D\right) \oplus D\right) \cup\left(\left(\left(X_{i}\right)_{d_{2}} \ominus D\right) \oplus D\right) \\
& \subset\left(X_{i}\right)_{d_{1}} \cup\left(X_{i}\right)_{d_{2}} \\
& \text { (pela Proposição 3.12). } \\
& \text { (uma vez que, para todo } h \in \mathbb{Z}^{2}, D \text { não é invariante de }\left(X_{i}\right)_{h} \text { ) } \\
& =X_{i+1} \text {. }
\end{aligned}
$$

Assim, $\left(X_{i+1} \ominus D\right) \oplus D$ é um subconjunto próprio de $X_{i+1}$. Mas isto é uma contradição, uma vez que, por hipótese, $D$ é um invariante de $X_{i+1}$, ou seja, $X_{i+1}=\left(X_{i+1} \ominus D\right) \oplus D$. Portanto, $D$ é um invariante de $X_{i}$.

Como uma conseqüência imediata da última proposição, temos o seguinte resultado.

Proposição 6.9 Seja $D$ um subconjunto do quadrado elementar. Para todo $i>0$,

(a) se $D$ é um invariante de $X_{i}$, então $D \equiv D_{1}$ ou $D \equiv D_{3}$;

(b) se $D$ é um invariante de $Y_{i}$, então $D \equiv D_{1}$ ou $D \equiv D_{4}$;

(c) se $D$ é um invariante de $Z_{i}$, então $D \equiv D_{1}$ ou $D \equiv D_{5}$.

Prova: Provaremos somente a primeira afirmação. As outras podem ser provadas de uma maneira similar.

A prova é por indução em $i$. Para o caso $i=1$, o subconjunto do quadrado elementar $D$ que é invariante de $X_{1}=D_{1} \oplus D_{3}$ é $D \equiv D_{1}$ ou $D \equiv D_{3}$ (fazendo-se uma busca exaustiva).

Agora, suponha por hipótese de indução que, para $i>0$, se $D$ é um invariante de $X_{i}$, então $D \equiv D_{1}$ ou $D \equiv D_{3}$. Provaremos que esta afirmação ainda vale para $X_{i+1}$.

Se $D$ é um invariante de $X_{i+1}$, então, pela Proposição 6.8, $D$ é também um invariante de $X_{i}$. Assim, por hipótese de indução, $D \equiv D_{1}$ ou $D \equiv D_{3}$.

Finalmente, o resultado principal desta seção.

Teorema 6.10 Para todo $i>0$, um elemento em qualquer decomposição de $X_{i}, Y_{i}$ e $Z_{i}$ não é simplesmente conexo.

Prova: Pela Proposição 6.9, os invariantes de $X_{i}$, que são subconjuntos do quadrado elementar, são somente $D_{1}^{\prime} \equiv D_{1}$ e $D_{3}^{\prime} \equiv D_{3}$. Assim, pelo Corolário 4.1, qualquer decomposição de $X_{i}$ pode conter somente $D_{1}^{\prime} \equiv D_{1}$ ou $D_{3}^{\prime} \equiv D_{3}$. Portanto, $X_{i} \equiv a D_{1} \oplus b D_{3}$, com $a \geq 0$ e $b \geq 0$. Assim, para provar que um elemento em qualquer decomposição de $X_{i}$ não 


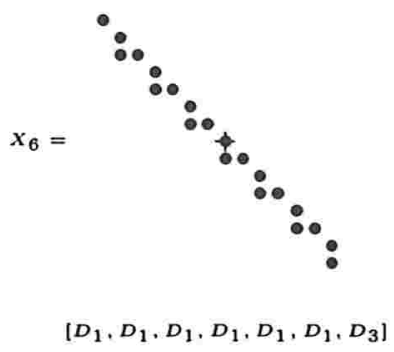

(a)

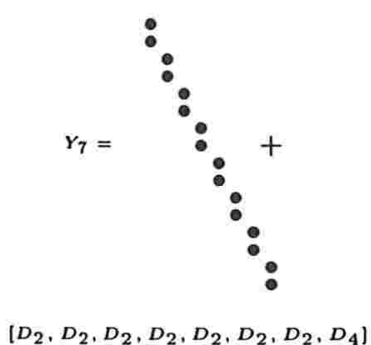

(b)

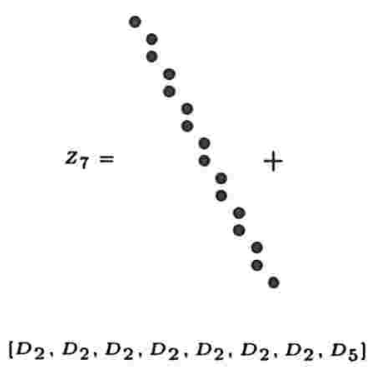

(c)

Figura 6.9: $(a)-(c)$ Os EE's $X_{6}, Y_{7}$ e $Z_{7}$ e suas respectivas decomposições.

é simplesmente conexo, devemos mostra que $a>0$, uma vez que $D_{1}$ não é simplesmente conexo (veja a Figura 6.4). Para isso, dividimos esta prova em duas partes. Na primeira, provamos que se $b=1$, então $a>0$. Na segunda parte, mostramos que $b=1$.

Provaremos agora que, se $b=1$ então $a>0$. Suponha por absurdo que $a=0$. Assim, $X_{i}=a D_{1} \oplus b D_{3}=D_{3}$, para todo $i>0$. Mas, isto é uma contradição, uma vez que, claramente, $X_{i} \not \equiv D_{3}$, para todo $i>0$. Dessa forma, se $b=1$, então $a>0$.

Provaremos agora que $b=1$.

Por um lado, $b \geq 1$. Suponha por absurdo que $b<1$ (isto é, $b=0$ ). Assim, $X_{i} \equiv a D_{1}$. Mas, isto é uma contradição, uma vez que, pelo Teorema 6.5, para todo $i>0, X_{i}$ é simplesmente conexo e $i D_{1}$ não é simplesmente conexo (veja a Figura 6.7). Logo, $b \geq 1$.

Por outro lado, $b \leq 1$. De fato, suponha por absurdo que $b>1$ (isto é, $b \geq 2$ ), então $X_{i}=a D_{1} \oplus b D_{3}=\left(a D_{1} \oplus(b-2) D_{3}\right) \oplus 2 D_{3}$ e conseqüentemente, pela Proposição 3.15 , $2 D_{3}$ é um invariante de $X_{i}$. Mas, isto é uma contradição, uma vez que, por inspeção, $2 D_{3}$ não é um invariante de $X_{1}$ e, portanto, pela Proposição $6.8,2 D_{3}$ não é um invariante de $X_{i}$, para todo $i>0$. Logo, $b \leq 1$.

Analogamente, pode-se mostrar que um elemento em qualquer decomposição de $Y_{i}$ e $Z_{i}$ não é simplesmente conexo.

Pela Proposição 6.10, as famílias de EE's simplesmente conexos $\mathcal{X}=\left\{X_{i}: i>0\right\}$, 
$\mathcal{Y}=\left\{Y_{i}: i>0\right\}$ e $\mathcal{Z}=\left\{Z_{i}: i>0\right\}$ não são decomponíveis segundo a definição de Park e Chin, uma vez que, pelo menos um elemento em qualquer decomposição $\left(D_{1}\right.$ ou $\left.D_{2}\right)$ desses EE's não é simplesmente conexo. Na Figura 6.9, mostramos um EE de cada família e suas respectivas decomposições.

\subsection{Complexidade do Algoritmo de Park e Chin}

No trabalho de Park e Chin [35] não foi mencionada a complexidade de tempo do algoritmo deles. No Passo 2 do algoritmo do Park e Chin, é necessário encontrar uma solução inteira de um sistema linear de inequações com um número fixo de variáveis $[35$, p. 8]. No entanto, eles não mostraram como fazer isso. Teoricamente, para cada número inteiro positivo $n$, existe um algoritmo polinomial para encontrar soluções inteiras de sistemas de inequações lineares com $n$ variáveis [40, p. 256]. No entanto, este resultado é somente teórico, e a implementação desse algoritmo na prática não é viável. No caso geral, resolver sistemas lineares de solução inteira é um problema bastante difícil [40, p. 227].

\subsection{Conclusão}

Neste capítulo, apresentamos três famílias infinitas de EE's simplesmente conexos tais que pelo menos um subconjunto do quadrado elementar em qualquer decomposição desses EE's não é simplesmente conexo. Isto significa que o algoritmo de Park e Chin é limitado no sentido de que ele não é capaz de decompor os EE's dessas famílias.

Teoricamente, o algoritmo de Park e Chin é um algoritmo polinomial, mas a sua implementação não é viável, uma vez que, no Passo 2 do algoritmo deles, é necessário encontrar uma solução inteira de um sistema linear com um número fixo de variáveis. 


\section{Capítulo 7}

\section{Decomposição de Elementos Estruturantes Arbitrários}

Este capítulo apresenta um algoritmo geral para decomposição de EE's arbitrários. Com este algoritmo é possível verificar se uma erosão (respectivamente, dilatação) arbitrária tem uma decomposição seqüencial. Se uma decomposição de um EE $A$ existe, então é apresentada uma decomposição ótima de $A$. O algoritmo usa a técnica de "branch and bound", com estratégias de poda baseadas em propriedades algébricas e geométricas da adição de Minkowski. A técnica aqui apresentada generaliza resultados clássicos como os de Zhuang and Haralick [51], Xu [49], e Park and Chin [35], com um desempenho melhor ou equivalente. Finalmente, análises teóricas do algoritmo proposto e resultados experimentais também são apresentados.

\subsection{Introdução}

Neste capítulo, apresentaremos um algoritmo para encontrar, se existe, uma decomposição de um EE arbitrário. Se uma tal decomposição existe, o algoritmo fornece uma decomposição com um número mínimo de elementos; caso contrário, ele mostra que o EE não tem uma decomposição.

Como no trabalho de Zhuang and Haralick [51], a idéia fundamental do método proposto é a aplicação de técnicas de Otimização Combinatória. A formulação adotada no algoritmo proposto neste capítulo é uma busca baseada em "branch and bound" em uma árvore que representa o espaço de todas as possíveis seqüências de subconjuntos que poderiam ser uma decomposição do EE de entrada. Nesta busca, o algoritmo procura soluções válidas, podando as soluções não viavéis. A eficiência de encontrar tais podas, que é baseada em propriedades algébricas e geométricas da adição de Minkowski, é essencial para a viabilidade do método. Se nenhuma solução válida é encontrada, então o EE não tem decomposição. 


\subsubsection{O Método "Branch and Bound"}

No Capítulo 4, Seção 4.1, vimos que o problema de encontrar uma decomposição de um EE $A$ equivale ao problema de encontrar uma subseqüência de Seqlnv[ $A]$.

Dado um EE $A$ tal que Seqlnv $[A]$ tenha um número grande de elementos, não é possível enumerar, em um tempo razoável, todas subseqüências de Seqlnv[ $A]$. Isto evidencia o fato de que não é possível encontrar uma decomposição de $A$ de comprimento mínimo por uma enumeração explícita de todas soluções possíveis. Assim, o problema de decompor um EE arbitrário é um problema de Otimização Combinatória.

Um método para enumerar parcialmente as possíveis soluções de um problema de Otimização Combinatória é conhecido como "branch and bound" [37, p. 40]. A idéia é particionar o conjunto de soluções possíveis em alguns conjuntos pequenos, gerando subproblemas independentes. Quando se resolve todos estes subproblemas, a melhor solução encontrada é a solução ótima do problema original. Esta partição pode ser vista como a construção de uma árvore, cujos nós correspondem às soluções parciais, e o nó raiz ao problema original. A busca nesta árvore pode ser melhorada se limites inferior e superior do valor da solução ótima são conhecidos. Por exemplo, se é conhecido uma solução viável cujo valor é, digamos, 25 e o nó atualmente visitado tem limite inferior igual a 28 , este ramo pode ser podado, uma vez que a melhor solução neste ramo não pode ser melhor que a melhor solução conhecida. A arte desta estratégia consiste em encontrar bons limites para evitar de visitar todas (ou a grande maioria) possíveis soluções do problema.

Há muitas maneiras de visitar os nós de uma árvore. No algoritmo apresentado na Seção 7.2, usamos a busca em profundidade [37, p. 39], que é descrita da seguinte forma. No início da busca, cada nó da árvore é marcado como "não visitado". Na primeira iteração, algum nó arbitrário é selecionado (se a árvore tem uma raiz, então a raiz é selecionada) e marcado como "visitado". Seus nós adjacentes são marcados como "alcançável" e colocados em uma pilha. Em uma iteração arbitrária, se a pilha está vazia, a busca termina. Caso contrário, o elemento que está no topo da pilha é desempilhado e marcado como "visitado". Os seus nós adjacentes que ainda estão marcados como "não visitado" são marcados como "alcançável" e colocados na pilha e uma nova iteração começa.

\subsubsection{A Árvore de Decomposição}

Dado um EE $A$, definimos uma árvore rotulada que representa o espaço de todas possíveis subseqüências de Seqlnv $[A]$. Seja $\left[B_{1}, B_{2}, \cdots, B_{m}\right]$ a seqüência de invariantes de $A$, isto é, Seqlnv $[A]=\left[B_{1}, B_{2}, \cdots, B_{m}\right]$. A árvore de decomposição de $A$, denotada $\mathcal{T}(A)$, é uma árvore rotulada tal que:

(1) Todos nós são rotulados por um subconjunto $Y=B_{i_{1}} \oplus B_{i_{2}} \oplus \cdots \oplus B_{i_{j}}$, onde $\left[B_{i_{1}}, B_{i_{2}}, \cdots, B_{i_{j}}\right]$ é uma subseqüência de Seqlnv $[A]$.

(2) O rótulo da raiz é o conjunto unitário que contém a origem e é denotado por $\{o\}$; 


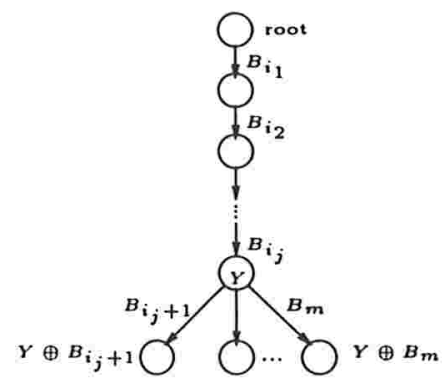

Figura 7.1: Um nó $Y$ na árvore de decomposição.

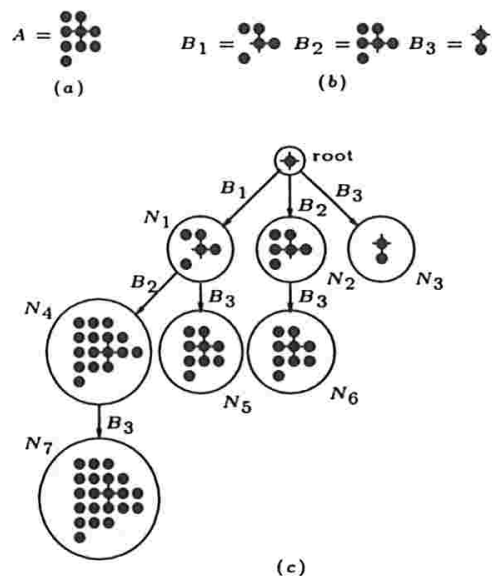

Figura 7.2: (a) Um EE $A$. (b) Seqüência de invariantes de $A$. (c) Árvore de decomposição de $A$.

(3) Os rótulos dos filhos de um nó cujo rótulo é $Y=B_{i_{1}} \oplus B_{i_{2}} \oplus \cdots \oplus B_{i_{j}}$ são $Y \oplus B_{i_{j}+1}$ (primeiro filho), $Y \oplus B_{i_{j}+2}$ (segundo filho), $\cdots, Y \oplus B_{m}$ (último filho). Para um exemplo, veja a Figura 7.1.

(4) A aresta que liga um nó cujo rótulo é $Y$ e seu filho cujo rótulo é $Y \oplus B_{k}$ é rotulada como $B_{k}$. (veja a Figura 7.1).

Freqüentemente, usaremos nó $Y$ significando nó cujo rótulo é $Y$.

Por construção da árvore de decomposição, não é difícil ver que, se a seqüência de invariantes de um dado $\mathrm{EE} A$ tem $m$ elementos, então $\mathcal{T}(A)$ tem $2^{m}$ nós. Para um exemplo de $\mathcal{T}(A)$, veja a Figura 7.2.

Seja $A$ um EE e sejam $X$ e $Y$ dois nós de $\mathcal{T}(A)$ tais que $X$ é um descendente de $Y$. Denotamos $\operatorname{Path}_{A}[Y, X]$ a seqüência de rótulos das arestas de $\mathcal{T}(A)$ que estão no caminho de $Y$ a $X$. Não é difícil ver que se $\operatorname{Path}_{A}[Y, X]=\left[C_{1}, C_{2}, \cdots, C_{j}\right]$, então $X=Y \oplus C_{1} \oplus$ $\cdots \oplus C_{j}$. Por exemplo, na Figura 7.2.c, Path $_{A}\left[N_{1}, N_{7}\right]=\left[B_{2}, B_{3}\right]$ e $N_{7}=N_{1} \oplus B_{2} \oplus B_{3}$. 


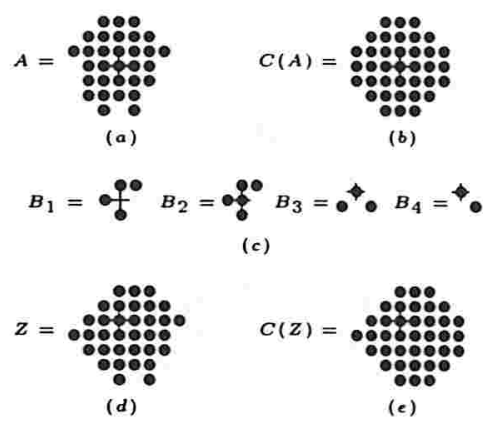

Figura 7.3: $(a)-(b)$ Um EE $A$ e $C(A)$. (c) Sequência de invariantes de $A$. $(d)-(e) Z$ e $C(Z)$.

Segundo esta notação, $\operatorname{Path}_{A}[\{o\}, Y]$ denota a seqüência de rótulos das arestas de $\mathcal{T}(A)$ que estão no caminho da raiz ao nó $Y$, que por sua vez forma uma subseqüência de Seqlnv $[A]$ cujo resultado da adição de Minkowski de todos elementos desta subseqüência é igual a $Y$. Por exemplo, na Figura 7.2.c, Path $_{A}\left[\{o\}, N_{6}\right]=\left[B_{2}, B_{3}\right]$ é uma subseqüência de Seqlnv $[A]$; e o nó $N_{6}=B_{2} \oplus B_{3}$. Assim, se o EE $A$ é igual a uma translação do nó $Y$ (isto é, $Y \equiv A$ ), então $A$ tem uma decomposição dada pela seqüência $\operatorname{Path}_{A}[\{o\}, Y]$.

O nível de um nó $Y$ na árvore de decomposição é o comprimento do caminho da raiz até $Y$ e é denotado por level $(Y)$. Desta forma, dado um EE $A$, o nosso objetivo é encontrar um nó $Y$ em $\mathcal{T}(A)$ tal que $Y \equiv A$ e seu nível seja mínimo. Se tal nó não existe, então o EE $A$ não é decomponível.

Seja $A$ um EE. Dado um nó $Y$ de $\mathcal{T}(A)$, dizemos que $Y$ é um nó viável se, e somente se, existe um nó $X$, descendente de $Y$, tal que $X \equiv A$. Por exemplo, na Figura 7.2.c, o nó $N_{1}$ é um nó viável, enquanto que o nó $N_{4}$ não é.

A arte da estratégia do algoritmo apresentado na Seção 7.2 é visitar os nós da árvore de decomposição, procurando por nós viáveis e "podando" os nós inviáveis.

\subsubsection{Condições Necessárias para Nós Viáveis}

Nesta subseção, mostraremos algumas condições necessárias para a existência de nós viáveis na árvore de decomposição de um dado EE $A$.

O resultado a seguir fornece uma condição necessária para a existência de uma decomposição para um dado EE.

Teorema 7.1 Seja $A$ um EE. Seja $Z$ o EE obtido pela adição de Minkowski de todos os elementos da seqüência Seqlnv $[A]$. Se existe $i \in\{0, \cdots, 7\}$ tal que $\nu_{i}(Z)<\nu_{i}(A)$, então $A$ não tem decomposição.

Prova: Suponha, por absurdo, que $A$ tem uma decomposição. Pela Proposição 4.3, existe uma subseqüência de Seqlnv $[A]$, digamos $\left[B_{1}, B_{2}, \cdots, B_{k}\right]$, que é uma decomposição 
de $A$. Logo, existe $h \in \mathbb{Z}^{2}$ tal que $A=\left(B_{1} \oplus B_{2} \oplus \cdots \oplus B_{k}\right)_{h}$. Assim, pela Proposição 4.16, $\nu(A)=\nu\left(B_{1}\right)+\nu\left(B_{2}\right)+\cdots+\nu\left(B_{k}\right)$. Conseqüentemente, $\nu_{j}(A)=\nu_{j}\left(B_{1}\right)+\nu_{j}\left(B_{2}\right)+\cdots+$ $\nu_{j}\left(B_{k}\right) \leq \nu_{j}(Z)$, para todo $j \in\{0,1, \cdots, 7\}$, uma vez que $Z$ é o resultado da adição de Minkowski de todos os elementos da seqüência Seqlnv $[A]$. Mas isto contradiz a hipótese de que existe $i \in\{0,1, \cdots, 7\}$ tal que $\nu_{i}(A)>\nu_{i}(Z)$. Portanto, $A$ não tem decomposição.

A Figura 7.3. $a$ apresenta um exemplo de um $\mathrm{EE} A$ que não tem decomposição. A seqüência de invariantes de $A$ está apresentada na Figura 7.3.c. Seja $Z$ o EE obtido pela adição de Minkowski de todos elementos em Seqlnv[A], isto é, $Z=B_{1} \oplus B_{2} \oplus B_{3} \oplus B_{4}$ (veja a Figura 7.3.d). O vetor de projeções de $A$ e $Z$ são, respectivamente, $\nu(A)=$ $(2,2,2,2,2,2,2,2)$ e $\nu(Z)=(2,2,2,2,2,3,0,3)$. Assim, pelo Teorema $7.1, A$ não tem decomposição, uma vez que $\nu_{6}(Z)<\nu_{6}(A)$.

O próximo teorema, uma conseqüência da Proposição 4.16, fornece uma condição para nós viáveis, e, portanto, podemos utilizá-la como uma estratégia para podar alguns nós não viáveis na árvore de decomposição de um dado EE.

Teorema 7.2 Seja A um EE. Seja $Y$ um nó de $\mathcal{T}(A)$. Se existe $i \in\{0, \cdots, 7\}$ tal que $\nu_{i}(Y)>\nu_{i}(A)$, então $Y$ não é um nó viável.

Prova: Suponha por absurdo que $Y$ é um nó viável. Assim, existe um descendente de $Y$, digamos $X$, tal que $X \equiv A$. Seja $W \subseteq \mathbb{Z}^{2}$ o conjunto obtido pela adição de Minkowski de todos elementos em $\operatorname{Path}_{A}[Y, X]$. Assim, $X=Y \oplus W \equiv A$, e, conseqüêntemente, existe $h \in \mathbb{Z}^{2}$ tal que $A=(Y \oplus W)_{h}$. Logo, pela Proposição 4.16, $\nu(A)=\nu(Y)+\nu(W)$. Desta forma, para todo $j \in\{0,1, \cdots, 7\}, \nu_{j}(A)=\nu_{j}(Y)+\nu_{j}(W)$, e, portanto, $\nu_{j}(A) \geq \nu_{j}(Y)$. Mas isto contradiz a hipótese de que existe $i \in\{0,1, \cdots, 7\}$ tal que $\nu_{i}(A)<\nu_{i}(Y)$. logo, $Y$ não é um nó viável.

Na Figura 7.2.c, os vetores projeção de $N_{4}$ e $A$ são, respectivamente, $\nu\left(N_{4}\right)=(2,2,0,2$, $2,0,4,0)$ e $\nu(A)=(1,1,1,1,1,0,3,0)$. Assim, pelo Teorema $7.2, N_{4}$ não é um nó viável, uma vez que $\nu_{0}\left(N_{4}\right)>\nu_{0}(A)$.

O teorema seguinte, que é uma conseqüência imediata da Proposição 3.15, é uma outra estratégia de poda (que também foi obtida por Zhuang e Haralick [51]).

Teorema 7.3 Seja A um EE. Seja $Y$ um nó de $\mathcal{T}(A)$. Se $Y$ não é um invariante de $A$, então $Y$ não é um nó viável.

As definições que se seguem são necessárias para se obter uma outra estratégia de poda (dada pelo Teorema 7.5).

Seja $A$ um EE. Seja $Y$ um nó de $\mathcal{T}(A)$. Denotaremos por $\operatorname{Arestas}_{A}[Y]$ a seqüência formada pelos rótulos das arestas que ligam $Y$ a seus filhos, isto é, se $Y$ é o nó raiz, então 
$\operatorname{Arestas}_{A}[Y]=$ Seqlnv $[A]$; se $Y=B_{i_{1}} \oplus \cdots \oplus B_{i_{j}}$, então $\operatorname{Arestas}_{A}[Y]=\left[B_{i_{j}+1}, B_{i_{j}+2}, \cdots, B_{m}\right]$ (veja a Figura 7.1). Por exemplo, na Figura 7.2.c, Arestas $_{A}\left[N_{1}\right]=\left[B_{2}, B_{3}\right]$. Se $X$ é um descendente de $Y$, então, por construção de $\mathcal{T}(A)$, a seqüência $\operatorname{Path}_{A}[Y, X]$ é uma subseqüência de $\operatorname{Arestas}_{A}[Y]$. Por exemplo, na Figura 7.2.c, Path ${ }_{A}\left[N_{1}, N_{5}\right]=\left[B_{3}\right]$ é uma subseqüência de $\operatorname{Arestas}_{A}\left[N_{1}\right]=\left[B_{2}, B_{3}\right]$.

Seja $A$ um EE. Seja $Y$ um nó de $\mathcal{T}(A)$. Definimos a subseqüência $\operatorname{Possível}_{A}[Y]$ da seqüência $\operatorname{Arestas}_{A}[Y]$ da seguinte forma. Seja $D_{1}, D_{2}, \cdots, D_{k}$ todos elementos distintos de $\operatorname{Arestas}_{A}[Y]$ tais que, para todo $i \in\{1,2, \cdots, k-1\}, D_{i}$ aparece antes de $D_{i+1}$ em $\operatorname{Arestas}_{A}[Y]$. Seja $m_{i}$ o número de ocorrências de $D_{i}$ em $\operatorname{Arestas}_{A}[Y]$. Claramente, $\operatorname{Arestas}_{A}[Y]=\operatorname{Seq}\left[D_{1}, m_{1}\right] \cdots \operatorname{Seq}\left[D_{k}, m_{k}\right]$. Seja $n_{i}$ o maior inteiro não negativo tal que $\left(Y \oplus n_{i} D_{i}\right)$ é um invariante de $A$. Seja $d_{i}=\min \left\{n_{i}, m_{i}\right\}$. A seqüência possível é definida como Possível ${ }_{A}[Y]=\operatorname{Seq}\left[D_{1}, d_{1}\right] \cdots \operatorname{Seq}\left[D_{k}, d_{k}\right]$. Por exemplo, na Figura 7.2.c, Possivel $_{A}\left[N_{1}\right]=B_{3}$, uma vez que Arestas $A\left[N_{1}\right]=B_{2}, B_{3}, N_{1} \oplus B_{2}=N_{4}$ não é um invariante de $A$ e $N_{1} \oplus B_{3}=N_{5}$ é um invariante de $A$.

No próximo resultado temos uma propriedade interessante para seqüências possíveis. Como conseqüência desta propriedade, obtemos uma nova estratégia de poda dada pelo Teorema 7.5.

Lema 7.4 Seja $A$ um EE. Sejam $X, Y$ dois nós de $\mathcal{T}(A)$ tais que $X$ é um descendente de $Y$. Se $X \equiv A$, então $\operatorname{Path}_{A}[Y, X]$ é uma subseqüência de $\operatorname{Possível}_{A}[Y]$.

Prova: Sejam $S_{1}, S_{2}, \cdots, S_{k}$ todos elementos distintos da seqüência Path Pa $_{A}[Y, X]$, tais que, para todo $i \in\{1,2, \cdots, k-1\}, S_{i}$ aparece antes de $S_{i+1}$ em $\operatorname{Path}_{A}[Y, X]$. Se $s_{i}$ é o número de ocorrências de $S_{i}$ na seqüência $\operatorname{Path}_{A}[Y, X]$, então $\operatorname{Path}_{A}[Y, X]=\operatorname{Seq}\left[S_{1}, s_{1}\right] \cdots$ Seq $\left[S_{k}, s_{k}\right]$. Assim, $X=Y \oplus s_{1} S_{1} \oplus s_{2} S_{2} \oplus \cdots \oplus s_{k} S_{k}$. Para provar que $\operatorname{Path}_{A}[Y, X]$ é uma subseqüência de $\operatorname{Possivel}_{A}[Y]$, temos que mostrar que:

(a) cada $S_{i} \in$ Possível $_{A}[Y]$;

(b) se $d_{i}$ é o número de ocorrências de $S_{i}$ na seqüência $\operatorname{Possível~}_{A}[Y]$, então $s_{i} \leq d_{i}$;

(c) para todo $i \in\{1, \cdots, k-1\}, S_{i}$ aparece antes de $S_{i+1}$ em $\operatorname{Possível}_{A}[Y]$.

Uma vez que $X=Y \oplus s_{1} S_{1} \oplus \cdots \oplus s_{k} S_{k} \equiv A$, existe $z \in \mathbb{Z}^{2}$ tal que $A=Y \oplus s_{1} S_{1} \oplus \cdots \oplus$ $s_{k} S_{k} \oplus\{z\}$. Desta forma, pela Proposição 3.15, $\left(Y \oplus s_{i} S_{i}\right)$ é um invariante de $A$ e, pela definição de $\operatorname{Possível}_{A}[Y], S_{i} \in \operatorname{Possível}_{A}[Y]$. Isto prova $(a)$.

Seja $n_{i}$ é o maior inteiro positivo tal que $\left(Y \oplus n_{i} S_{i}\right)$ é invariante de $A$. Seja $m_{i}$ o número de ocorrências de $S_{i}$ em $\operatorname{Arestas}_{A}[Y]$. Pela construção da seqüência $\operatorname{Possível}_{A}[Y]$, o número de ocorrências de $S_{i}$ em Possível ${ }_{A}[Y]$ é $d_{i}=\min \left\{n_{i}, m_{i}\right\}$. Se $d_{i}=n_{i}$, então $s_{i} \leq n_{i}$, uma vez que $Y \oplus s_{i} S_{i}$ é invariante de $A$. Se $d_{i}=m_{i}$, then $s_{i} \leq m_{i}$, uma vez que $\operatorname{Path}_{A}[Y, X]$ é uma subseqüência de $\operatorname{Arestas}_{A}[Y]$. Isto prova $(b)$. 
Suponha por absurdo que existe $j \in\{1,2, \cdots, k-1\}$ tal que $S_{j+1}$ aparece antes de $S_{j}$ em Possível $_{A}[Y]$. Uma vez que a seqüência $\operatorname{Possivel}_{A}[Y]$ é uma subseqüência de $\operatorname{Arestas}_{A}[Y]$, então $S_{j+1}$ aparece antes de $S_{j}$ em $\operatorname{Arestas}_{A}[Y]$. Uma vez que $\operatorname{Path}_{A}[Y, X]$ é uma subseqüência de $\operatorname{Arestas}_{A}[Y]$, então $S_{j+1}$ aparece antes de $S_{j}$ em $\operatorname{Path}_{A}[Y, X]$. Mas isto é uma contradição, uma vez que, por construção de $\operatorname{Path}_{A}[Y, X], S_{j}$ aparece antes de $S_{j+1}$ em Path $_{A}[Y, X]$. Portanto, para todo $i \in\{1,2, \cdots, k-1\}, S_{i}$ aparece antes de $S_{i+1}$ na seqüência $\operatorname{Possível}_{A}[Y]$. Isto prova $(c)$.

O teorema seguinte fornece uma nova estratégia de poda.

Teorema 7.5 Seja $A$ um EE. Seja $Y$ um nó de $\mathcal{T}(A)$. Seja $Z \subseteq \mathbb{Z}^{2}$ o conjunto obtido pela adição de Minkowski de todos os elementos de Possivel $_{A}[Y]$. Se existe $i \in\{0, \cdots, 7\}$ tal que $\nu_{i}(Z)<\nu_{i}(A)-\nu_{i}(Y)$, então $Y$ não é um nó viável.

Prova: Suponha por absurdo que $Y$ é um nó viável. Assim, existe um descendente de $Y$, digamos $X$, tal que $X \equiv A$. Seja $W \subseteq \mathbb{Z}^{2}$ o conjunto obtido pela adição de Minkowski de todos elementos em Path $_{A}[Y, X]$. Dessa forma, $X=Y \oplus W \equiv A$, e, conseqüentemente, existe $h \in \mathbb{Z}^{2}$ tal que $A=(Y \oplus W)_{h}$. Logo, pela Proposição 4.16, temos que $\nu(A)=$ $\nu(Y)+\nu(W)$. Uma vez que, pelo Lema 7.4, a seqüência $\operatorname{Path}_{A}[Y, X]$ é uma subseqüência de Possível $_{A}[Y]$, então, para todo $j \in\{0, \cdots, 7\}, \nu_{j}(Z) \geq \nu_{j}(W)=\nu_{j}(A)-\nu_{j}(Y)$. Mas, isto é uma contradição, pois, por hipótese, existe $i \in\{0, \cdots, 7\}$ tal que $\nu_{i}(Z)<\nu_{i}(A)-\nu_{i}(Y)$. Portanto, $Y$ não é um nó viável.

Com as estratégias dadas pelos Teoremas 7.2, 7.3 e 7.5, podemos podar alguns (possivelmente, não todos) nós inviáveis da árvore de decomposição. Apresentamos na próxima seção o algoritmo que faz uso dessas propriedades de poda.

\subsection{Decomposição Ótima de um Elemento Estrutu- rante}

Nesta seção, apresentaremos um algoritmo para encontrar uma decomposição ótima de um EE $A$ arbitrário.

A proposição seguinte caracteriza um nó $Y$ na árvore de decomposição de um dado EE $A$ tal que $Y \equiv A$. Um resultado análogo, usando vetor retangular em vez do vetor projeção, foi apresentado na Proposição 5.7.

Proposição 7.6 Seja $A$ um EE e seja $Y$ um nó de $\mathcal{T}(A)$. Então, $Y \equiv A$ se, e somente se, $Y$ é um invariante de $A$ e $\nu(Y)=\nu(A)$.

Prova: $(\Rightarrow)$ Se $Y \equiv A$, então, existe $h \in \mathbb{Z}^{2}$ tal que $Y=A_{h}=A \oplus\{h\}$. Logo, pela Proposiçào 3.15, $Y$ é um invariante de $A$. Uma vez que $\nu\left(A_{h}\right)=\nu(A)$, então, $\nu(Y)=\nu(A)$. 
$(\Leftarrow)$ Uma vez que $Y$ é um invariante de $A$, pela Proposição 3.14, existe um EE $X$ tal que $A=X \oplus Y$. Pela Proposição 4.16, $\nu(A)=\nu(X)+\nu(Y)$. Uma vez que $\nu(A)=\nu(Y)$, então $\nu(X)=0$ e, portanto, $|X|=1$. Seja $h \in \mathbb{Z}^{2}$ tal que $X=\{h\}$. Neste caso, a adição de Minkowski $X \oplus Y=A$ é uma translação de $Y$ por $h$. Logo, $Y \equiv A$.

Seja $A$ um EE. Quando encontramos um nó $Y$ de $\mathcal{T}(A)$ tal que $Y$ é invariante de $A$ e $\nu(Y)=\nu(A)$, então, pela Proposição 7.6, $Y \equiv A$, e, portanto, a seqüência $\operatorname{Path}_{A}[\{o\}, Y]$ é uma decomposição de $A$. Se level $(Y)$ é igual a lower $(A)$ (o limite inferior estabelecido pelo Teorema 4.18), então $\operatorname{Path}_{A}[\{o\}, Y]$ é uma solução ótima. Caso contrário, a solução ótima contém no máximo level $(Y)$ elementos. Assim, acabamos de obter um limite superior para a decomposição de $A$. Note que este limite superior pode mudar dinamicamente. $\mathrm{O}$ limite superior atual é denotado por $l s a$.

Uma tarefa crucial para o algoritmo é uma poda adequada na árvore de decomposição quando seus nós estão sendo visitados. O Teorema 7.1 fornece uma condição necessária para a existência de uma decomposição de $A$. Assim, devemos começar a busca verificando se esta condição é satisfeita. O Teorema 4.18 fixa o limite inferior para uma decomposição de $A$, enquanto os Teoremas $7.2,7.3$ e 7.5 guarantem podas importantes, uma vez que eles detectam nós inviáveis.

Neste contexto, o algoritmo verifica se a condição necessária para a existência de uma decomposição é satisfeita. Se ela não é satisfeita, então o EE não tem decomposição e o algoritmo pára. Caso contrário, depois de inicializar lsa $\leftarrow \infty$, a busca de uma decomposição começa. Na primeira iteração, o nó raiz é selecionado e marcado como "visitado" e seus filhos são colocados em uma pilha. Em uma iteração arbitrária, se a pilha está vazia, então o algoritmo verifica dois casos: $(i)$ se lsa $=\infty$, então o EE $A$ não tem decomposição; (ii) se lsa $=\operatorname{level}(Y)$, então $\operatorname{Path}_{A}[\{o\}, Y]$ é uma decomposição ótima de $A$. Nos dois casos, a busca termina. Se a pilha não está vazia, o elemento do topo, digamos $Y$, é desempilhado e marcado como "visitado" e o algoritmo verifica se o nó $Y$ pode ser podado ou se ele é uma solução, ou seja, se $Y \equiv A$.

\section{(i) - Verificar se o nó $Y$ pode ser podado.}

Temos quatro estratégias de poda que devem ser examinadas:

(i.a) Poda por limite superior.

Se level $(Y) \geq l$ sa.

(i.b) Poda por projeção.

Se existe $i$ tal que $\nu_{i}(Y)>\nu_{i}(A)$.

(i.c) Poda por invariança.

Se $Y$ não é invariante de $A$.

(i.d) poda por seqüência possível.

Seja $Z \subseteq \mathbb{Z}^{2}$ o conjunto obtido pela adição de Minkowski de todos os elementos da seqüência Possível $_{A}[Y]$. 
Se existe $i \in\{0,1, \cdots, 7\}$ tal que $\nu_{i}(Z)<\nu_{i}(A)-\nu_{i}(Y)$.

Se uma dessas condições é satisfeita, então o nó $Y$ é podado e começa-se uma nova iteração.

(ii) - Verificar se o nó $Y$ é uma solução.

$Y$ é uma solução se as duas condições seguintes são satisfeitas simultaneamente:

- $\operatorname{level}(Y) \geq \operatorname{lower}(A) \mathrm{e}$

- $\nu(Y)=\nu(A)$.

No caso dessas duas condições serem satisfeitas, $Y$ é um invariante de $A$ (caso contrário, $Y$ seria podado no passo $(i . c)$ ) e, uma vez que $\nu(Y)=\nu(A)$, pela Proposição 7.6, $Y \equiv A$, e, portanto, $\operatorname{Path}_{A}[\{o\}, Y]$ é uma decomposição de $A$.

(ii.a) - O nó Y é uma solução.

Temos duas possibilidades:

- $\quad$ se $\operatorname{level}(Y)=\operatorname{lower}(A)$

$\Rightarrow$ A busca termina.

- $\operatorname{se} \operatorname{level}(Y)>\operatorname{lower}(A)$

$\Rightarrow l s a \leftarrow \operatorname{level}(Y)$

e começa-se uma nova iteração.

(ii.b) - O nó Y não é uma solução.

Os filhos de $Y$ são empilhados começa-se uma nova iteração.

Na Figura 7.4, apresentamos um exemplo simples de aplicação do algoritmo para encontrar uma decomposição ótima do $\mathrm{EE} A$ mostrado na Figura 7.4.a. A seqüência de invariantes de $A$ é apresentada na Figura 7.4.b. O vetor projeção, o vetor retangular e o limite inferior de $A$ estão mostrados na Figura 7.4.c. O nó raiz é selecionado para ser visitado na Figura 7.4.d. O nó $N_{1}$ está sendo visitado na Figura 7.4.e. O nó $N_{4}$ está sendo visitado na Figura 7.4.f. O nó $N_{4}$ é podado (poda por projeção, veja Teorema 7.2) na Figura 7.4.g. $\mathrm{O}$ nó $N_{5}$ está sendo visitado na Figura 7.4.h. Uma vez que $N_{5} \equiv A$, então $\operatorname{Path}_{A}\left[\{o\}, N_{5}\right]=\left[B_{1}, B_{3}\right]$ é uma decomposição de $A$. Além disso, uma vez que level $\left(N_{5}\right)=2=\operatorname{lower}(A)$, então $\operatorname{Path}_{A}\left[\{o\}, N_{5}\right]$ é uma solução ótima e a busca pára. Neste exemplo particular, somente a poda de projeção foi detectada. Em geral, as outras estratégias de podas são detectadas de uma maneira similar.

Note que o limite inferior, dado pelo Teorema 4.18, pode não ser atingido, ou seja, pode existir um EE $A$ tal que o número de elementos em uma decomposição ótima de $A$ é estritamente maior que lower $(A)$. Mas, se a entrada do algoritmo é um EE convexo, o Teorema 5.14, mostrado no Capítulo 5, guarante que este limite inferior sempre é alcançado.

A seguir faremos um comentário da complexidade do algoritmo proposto. 


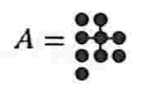

(a)

$$
B_{1}=\text { \% } B_{2}=\text { \&ै } B_{3}=\text { के }
$$

(b)

$$
\begin{gathered}
\nu(A)=(1,1,1,1,1,0,3,0) \\
\\
\rho(A)=(2,3) \\
\text { lower }(A)=2
\end{gathered}
$$

(c)
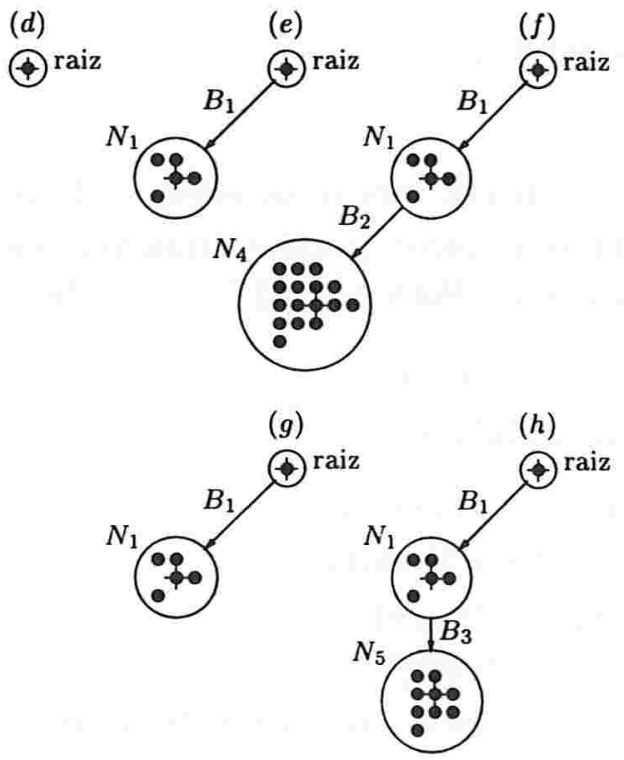

Figura 7.4: Aplicação do algoritmo para um exemplo simples.

Proposição 7.7 Seja A um EE. Se m é o número de elementos na seqüência de invariantes de $A$, então $m=O(n)$, onde $n=\max \left\{\rho_{0}(A), \rho_{1}(A)\right\}$.

Prova: Claramente, a multiplicidade de um dado EE com respeito a $A$ é no máximo $\max \left\{\rho_{0}(A), \rho_{1}(A)\right\}=O(n)$ e o número de subconjuntos do quadrado elementar é $2^{9}$. Assim, o número de elementos na seqüência Seqlnv $[A]$ é no máximo $2^{9} \cdot \max \left\{\rho_{1}(A), \rho_{2}(A)\right\}=$ $O(n)$. Portanto, $m=O(n)$.

Uma vez que a árvore de decomposição de um dado EE $A$ contém $2^{m}$ nós, onde $m$ é o número de elementos na seqüência de invariantes de $A$, e pela Proposição 7.7, $m=O(n)$, onde $n=\max \left\{\rho_{0}(A), \rho_{1}(A)\right\}$. Dessa forma, a complexidade do algoritmo proposto é, no pior caso, $O\left(2^{n}\right)$. 


\subsection{Comparação com Alguns Algoritmos Conhecidos}

Nesta seção, compararemos o algoritmo apresentado na Seção 7.2 com alguns algoritmos conhecidos na literatura. A Subseção 7.3.1 compara com o algoritmo de Zhuang e Haralick [51] que decompõe EE's arbitrários. A Subseção 7.3.2 compara com o algoritmo do $\mathrm{Xu}$ [49] que decompõe EE's convexos. E a Subseção 7.3 .3 compara com o algoritmo de Park e Chin [35] que decompõe EE's simplesmente conexos.

\subsubsection{Algoritmo de Zhuang e Haralick}

Zhuang e Haralick [51] apresentaram um algoritmo para encontrar uma decomposição ótima de um EE arbitrário onde todos elementos nesta decomposição têm um número $k$ pré-fixado de pontos. O algoritmo deles faz uma busca em largura em uma árvore (que eles chamaram árvore de busca e a técnica de solução utilizada por eles é dividida em duas partes: (a) os pontos de um EE que participa de uma decomposição do EE de entrada $A$ deve ser a diferença entre os pontos de $A$, isto é, se $B$ está em uma decomposição de $A$, então, para todo $z \in B$, existem $x, y \in A$ tais que $z=x-y$; e $(b)$ a redução do espaço de busca é feita usando a mesma estratégia dada pelo Teorema 7.3. Eles chamaram esta estratégia de verificação para frente.

A busca em largura tem vantagens e desvantagens em relação à busca em profundidade. A principal vantagem é que a primeira solução encontrada é sempre ótima. A principal desvantagem é que todos nós de um nível corrente devem estar guardados na memória. No algoritmo proposto, decidimos usar a busca em profundidade pois ele utiliza menos memória e, em nossos experimentos (veja a Seção 7.4), observamos que normalmente a distância entre a solução ótima e a primeira solução encontrada é pequena.

O problema de decomposição considerado neste trabalho (definido no Capítulo 3, Seção 3.8.1) é um caso especial do problema estudado por Zhuang e Haralick (isto é, $k=2,3, \cdots, 9$ e cada EE na decomposição é um subconjunto do quadrado elementar). Neste caso particular, o algoritmo proposto na Seção 7.2 consegue uma maior redução no espaço de busca, uma vez que ele usa mais duas estratégias de poda que o algoritmo de Zhuang e Haralick (podas por projeção e por seqüência possível).

\subsubsection{Algoritmo do $\mathrm{Xu}$}

$\mathrm{Xu}$ [49] desenvolveu um algoritmo para encontrar uma decomposição ótima de EE's convexos em termos de subconjuntos do quadrado elementar, onde todos elementos nesta decomposição são também convexos (veja o Capítulo 5).

Dado um EE convexo, a complexidade do algoritmo do Xu é $O\left(n^{2}\right)$, onde $n$ é o valor máximo entre as coordenadas do vetor retangular de $A$, ou seja, $=\max \left\{\rho_{0}(A), \rho_{1}(A)\right\}$. Nesta subseção, vamos mostrar que, se a entrada do nosso algoritmo é um EE convexo, 
então ele encontra uma decomposição ótima de $A$ em tempo $O\left(n^{4}\right)$. Além disso, esta decomposição pode conter EE's não convexos (neste sentido, o algoritmo proposto é mais geral que o algoritmo do $\mathrm{Xu}$ ).

Dado um EE $A$, dependendo da ordem escolhida para construir a seqüência SeqQ (ou equivalentemente, da ordem escolhida para os elementos de Seqlnv $[A]$ ), diferentes heurísticas de busca para se encontrar uma decomposição de $A$ podem ser obtidas. Para este algoritmo, utilizamos a mesma ordem estabelecida no Capítulo 5, Seção 5.3, ou seja, em ordem decrescente segundo a soma das coordenadas dos vetores retangular de cada elemento em SeqQ (conseqüentemente em Seqlnv[A]), isto é, $\rho_{0}\left(B_{1}\right)+\rho_{1}\left(B_{1}\right) \geq \rho_{0}\left(B_{2}\right)+$ $\rho_{1}\left(B_{2}\right) \geq \cdots \geq \rho_{0}\left(B_{n}\right)+\rho_{1}\left(B_{n}\right)$, e, ao mesmo tempo, se $\rho_{0}\left(B_{i}\right)+\rho_{1}\left(B_{i}\right)=\rho_{0}\left(B_{j}\right)+\rho_{1}\left(B_{j}\right)$ e $\left|B_{i}\right| \leq\left|B_{j}\right|$, então $B_{i}$ aparece antes que $B_{j}$ em SeqQ (conseqüentemente em Seqlnv $[A]$ ) (veja um exemplo na Figura 5.1).

Dado um EE $A$ e um nó $Y$ de $\mathcal{T}(A)$, seja $D$ o primeiro elemento em Arestas $_{A}[Y]$ tal que $Y \oplus D$ é um invariante de $A$. Definimos o nó $Y \oplus B$ como o nó invariante mais à esquerda de $Y$. Definimos a seqüência de nós mais à esquerda da árvore de decomposição $\mathcal{T}(A)$ como a seqüência $\left[T_{0}, T_{1}, T_{2}, \cdots, T_{j}\right]$ formada pelos nós de $\mathcal{T}(A)$ tais que $T_{0}$ é o nó raiz (isto é, um conjunto unitário que contém a origem) e, para todo $i=1,2, \ldots, j$, $T_{i}$ é o nó invariante mais à esquerda de $T_{i-1}$. Dizemos que a seqüência de nós mais à esquerda $\left[T_{0}, T_{1}, T_{2}, \cdots, T_{j}\right]$ de $\mathcal{T}(A)$ é maximal se, e somente se, para todo elemento $B$ $\operatorname{de}_{\operatorname{Arestas}}\left[T_{j}\right], T_{j} \oplus B$ não é um invariante de $A$.

Agora, vamos mostrar que, dado um EE convexo $A$, se a seqüência de nós mais à esquerda de $\mathcal{T}(A)$, digamos $\left[T_{0}, T_{1}, T_{2}, \cdots, T_{j}\right]$, é maximal, então a seqüência $\operatorname{Path}_{A}\left[\{o\}, T_{j}\right]=$ $\left[D_{1}, D_{2}, \cdots, D_{j}\right]$ é uma decomposição ótima de $A$. Para isso, dividiremos a nossa prova em duas partes. Primeiramente, vamos provar que $\operatorname{Path}_{A}\left[\{o\}, T_{j}\right]$ é uma decomposição de $A$, ou seja, que $T_{j} \equiv A$ e, posteriormente, que $\operatorname{Path}_{A}\left[\{o\}, T_{j}\right]$ é uma decomposição ótima, ou seja, $\operatorname{lower}(A)=j$.

\subsubsection{A Seqüência Maximal de Nós mais à Esquerda é uma Decomposição}

Agora, vamos mostrar, no Teorema 7.12, que, dado um EE $A$, se a seqüência de nós mais à esquerda de $\mathcal{T}(A)$, digamos $\left[T_{0}, T_{1}, \cdots, T_{j}\right]$, é maximal, então a seqüência $\operatorname{Path}_{A}\left[\{o\}, T_{j}\right]$ é uma decomposição de $A$. Para isso, precisamos de alguns resultados preliminares.

Lema 7.8 Seja $A$ um EE. Seja $\left[T_{0}, T_{1}, \cdots, T_{j}\right]$ a seqüência de nós mais à esquerda de $\mathcal{T}(A)$. Se $j>0$ e $T_{j} \oplus D$ é um invariante de $A$, então $T_{j-1} \oplus D$ também é um invariante de $A$.

Prova: Uma vez que $T_{j} \oplus D$ é um invariante de $A$, então, pela Proposição 3.14 , existe $Z \in \mathbb{Z}^{2}$ tal que $\left(T_{j} \oplus D\right) \oplus Z=A$. Como $T_{j}=T_{j-1} \oplus D_{j}$, então $\left(\left(T_{j-1} \oplus D_{j}\right) \oplus D\right) \oplus Z=A$. Pela propriedade de comutatividade e associatividade da adição de Minkowski temos que 
$\left(T_{j-1} \oplus D\right) \oplus\left(D_{j} \oplus Z\right)=A$. E finalmente, pela Proposição $3.15, T_{j-1} \oplus D$ é um invariante de $A$.

Lema 7.9 Seja $A$ um EE. Seja $\left[T_{0}, T_{1}, \cdots, T_{j}\right]$ a seqüência de nós mais à esquerda de $\mathcal{T}(A)$. Seja $D$ um elemento da seqüência Seqlnv $[A]=\left[B_{1}, B_{2}, \cdots, B_{n}\right] . \quad S e T_{j} \oplus D$ é um invariante de $A$, então $D$ é um elemento de $\operatorname{Arestas}_{A}\left[T_{j}\right]$.

Prova: Suponha por absurdo que $D \notin \operatorname{Arestas}_{A}\left[T_{j}\right]$. Seja $m$ a multiplicidade de $D$ com respeito a $A$. Como $\operatorname{Arestas}_{A}\left[T_{0}\right]=\operatorname{Seq} \operatorname{lnv}[A]$ e $D \in \operatorname{Seq} \operatorname{lnv}[A]$, então existe $i \in$ $\{1,2, \cdots, j\}$ tal que $\operatorname{Arestas}_{A}\left[T_{i-1}\right]=\left[B_{a}, B_{a+1}, \cdots, B_{b}\right] \cdot \operatorname{Seq}[D, m] \cdot\left[B_{c}, B_{c+1}, \cdots, B_{n}\right]$ e $D$ é o primeiro elemento de $\operatorname{Arestas}_{A}\left[T_{i-1}\right]$ tal que $T_{i-1} \oplus D$ é um invariante de $A$, isto é, para todo elemento $B \in\left[B_{a}, B_{a+1}, \cdots, B_{b}\right], T_{i-1} \oplus B$ não é invariante de $A$.

Seja $\left[D_{1}, D_{2}, \cdots, D_{j}\right]$ a seqüência dos rótulos das arestas que estão no caminho do nó raiz até o nó $T_{j}$. Assim, para cada $\ell \in\{1,2, \cdots, j\}, D_{\ell}$ é o primeiro elemento de $\operatorname{Arestas}_{A}\left[T_{\ell-1}\right]$ tal que $T_{\ell-1} \oplus D_{\ell}$ é um invariante de $A$.

Uma vez que $T_{j} \oplus D$ é um invariante de $A$, então, podemos inferir pelo Lema 7.8 que, para todo $\ell \in\{1,2, \cdots, j\}, T_{\ell-1} \oplus D$ é um invariante de $A$. Dessa forma, pela definição da seqüência de nós mais à esquerda, $D_{i}=D, D_{i+1}=D, \cdots, D_{i+m-1}=D$. Logo, $i+m-1 \leq j$ e $T_{i}=T_{i-1} \oplus D_{i}=T_{i-1} \oplus D, T_{i+1}=T_{i} \oplus D_{i+1}=T_{i} \oplus D=T_{i-1} \oplus 2 D, \cdots$, $T_{i+m-1}=T_{i-1} \oplus m D, T_{i+m}=T_{i-1} \oplus m D \oplus D_{i+m}, \cdots, T_{j}=T_{i-1} \oplus m D \oplus D_{i+m} \oplus \cdots \oplus D_{j}$. Portanto, se $Z=T_{i-1} \oplus D_{i+m} \oplus \cdots \oplus D_{j}$, então $T_{j}=Z \oplus m D$.

Como $T_{j} \oplus D$ é invariante de $A$, então, pela Proposição 3.14, existe um conjunto $T \in \mathbb{Z}^{2}$ tal que $\left(T_{j} \oplus D\right) \oplus T=A$. Como $T_{j}=Z \oplus m D$, então, $(Z \oplus T) \oplus(m+1) D=A$. Assim, pela Proposição $3.15,(m+1) D$ é invariante de $A$. Mas isto contradiz a definição de multiplicidade de $A$. Logo, $D \in \operatorname{Arestas}_{A}\left[T_{j}\right]$

Lema 7.10 Seja $A$ um EE convexo. A seqüência de nós mais à esquerda de $\mathcal{T}(A)$, digamos $\left[T_{0}, T_{1}, \cdots, T_{j}\right]$, é maximal se, e somente se, $\rho\left(T_{j}\right)=\rho(A)$.

Prova: $\quad(\Leftarrow)$ Suponha por absurdo que a seqüência de nós mais à esquerda de $\mathcal{T}(A)$ não seja maximal. Seja $B$ um elemento de $\operatorname{Arestas}_{A}\left[T_{j}\right]$ tal que $T_{j} \oplus B$ é um invariante de $A$.

Uma vez que $\operatorname{Arestas}_{A}\left[T_{j}\right]$ é uma subseqüência de Seqlnv $[A]$, então $B$ tem pelo menos dois pontos e, conseqüentemente, $\rho_{0}(B)>0$ ou $\rho_{1}(B)>0$.

Uma vez que $T_{j} \oplus B$ é um invariante de $A$, então, pela Proposição 3.14, existe um EE $X$ tal que $A=X \oplus\left(T_{j} \oplus B\right)$. Assim, pela Proposição 4.17, $\rho(A)=\rho(X)+\rho\left(T_{j}\right)+\rho(B)$. Como $\rho\left(T_{j}\right)=\rho(A)$, logo, $\rho(X)+\rho(B)=(0,0)$ e, portanto, $\rho_{0}(B)=0$ e $\rho_{1}(B)=0$. Mas isto contradiz o fato de que $\rho_{0}(B)>0$ ou $\rho_{1}(B)>0$. Portanto, a seqüência de nós mais à esquerda de $\mathcal{T}(A)$ é maximal.

$(\Rightarrow)$ Suponha por absurdo que $\rho\left(T_{j}\right) \neq \rho(A)$. Obviamente, por construção da seqüência de nós mais à esquerda, $T_{j}$ é um invariante de $A$. 
Como $A$ é um EE convexo e $T_{j}$ é um invariante de $A$, então, pelo Lema 5.6, $A \ominus T_{j}$ é também um EE convexo. Assim, pela Proposição 5.1, $A \ominus T_{j}$ tem uma decomposição seqüencial, digamos $\left[Z_{1}, Z_{2}, \cdots, Z_{\ell}\right]$. Como $T_{j}$ é um invariante de $A$, então, pela Proposição 5.19, $\rho\left(A \ominus T_{j}\right)=\rho(A)-\rho\left(T_{j}\right)$ Uma vez que $\rho\left(T_{j}\right) \neq \rho(A)$, então $\rho\left(A \ominus T_{j}\right) \neq(0,0)$. Assim, $\left|A \ominus T_{j}\right| \geq 2$, e, conseqüentemente, um elemento em $\left[Z_{1}, Z_{2}, \cdots, Z_{\ell}\right]$, digamos $Z_{1}$, contém pelo menos dois pontos. Claramente, $Z_{1}$ é um invariante de $A \ominus T_{j}$. Uma vez que $Z_{1}$ é um subconjunto do quadrado elementar e $\left|Z_{1}\right| \geq 2$, então existe um elemento $B$ em Seqlnv $[A]=\left[B_{1}, B_{2}, \cdots, B_{n}\right]$ tal que $B \equiv Z_{1}$. Como $Z_{1}$ é um invariante de $A \ominus T_{j}$, obviamente, $B$ também é um invariante de $A \ominus T_{j}$. Portanto, pela Proposição 3.14, existe um conjunto $X$ tal que $B \oplus X=A \ominus T_{j}$.

Uma vez que, $T_{j}$ é um invariante de $A$, então, $\left(A \ominus T_{j}\right) \oplus T_{j}=A$. Logo, $(B \oplus X) \oplus T_{j}=$ $X \oplus\left(T_{j} \oplus B\right)=A$. Dessa forma, pela Proposição 3.15, $T_{j} \oplus B$ é um invariante de $A$. Portanto, pelo Lema $7.9, B$ é um elemento de $\operatorname{Arestas}_{A}\left[T_{j}\right]$. Mas isto contradiz a hipótese de que a seqüência de nós mais à esquerda de $\mathcal{T}(A)$ é maximal. Portanto, $\rho\left(T_{j}\right)=\rho(A)$.

Lema 7.11 Seja $A$ um EE convexo. A seqüência de nós mais à esquerda de $\mathcal{T}(A)$, digamos $\left[T_{0}, T_{1}, \cdots, T_{j}\right]$, é maximal, então $T_{j} \equiv A$.

Prova: Por construção da seqüência de nós mais à esquerda, $T_{j}$ é um invariante de $A$. Pelo Lema 7.10, $\rho\left(T_{j}\right)=\rho(A)$. Logo, pela Proposição 5.7, $T_{j} \equiv A$.

Teorema 7.12 Seja $A$ um EE convexo. A seqüência de nós mais à esquerda de $\mathcal{T}(A)$, digamos $\left[T_{0}, T_{1}, \cdots, T_{j}\right]$, é maximal, então a seqüência Path $_{A}\left[\{o\}, T_{j}\right]$ é uma decomposição de $A$.

Prova: $\quad$ Seja Path $A\left[\{o\}, T_{j}\right]=\left[D_{1}, D_{2}, \cdots, D_{j}\right]$. Por construção, $T_{j}=D_{1} \oplus D_{2} \oplus \cdots \oplus D_{j}$. Pelo Lema 7.11, $T_{j} \equiv A$. Portanto, Path $_{A}\left[\{o\}, T_{j}\right]$ é uma decomposição de $A$.

\subsubsection{A Seqüência Maximal de Nós mais à Esquerda é uma Decomposição Ótima}

Agora, vamos provar que, dado um $\mathrm{EE}$ convexo $A$, se $\left[T_{0}, T_{1}, \cdots, T_{j}\right]$ é uma seqüência maximal de nós mais à esquerda de $\mathcal{T}(A)$, então $\left[T_{0}, T_{1}, \cdots, T_{j}\right]$ é uma decomposição ótima de $A$. Para isso, mostraremos que $\operatorname{lower}(A)=j$. Como feito na Subseção 5.5.3, a Proposição 7.13 (cuja prova está na Seção 7.6) mostra que $\left[T_{0}, T_{1}, \cdots, T_{j}\right]$ é uma seqüência elemento retangular de $A$, e, dessa forma, pela Proposição 5.16, $\operatorname{lower}\left(T_{j}\right)=j$. Como, pela Proposição $7.11, T_{j} \equiv A$, então, claramente, $\operatorname{lower}(A)=j$. 
Proposição 7.13 Se $A$ é um EE convexo, então uma seqüência de nós mais à esquerda de $\mathcal{T}(A)$, digamos $\left[T_{0}, T_{1}, \cdots, T_{j}\right]$, é uma seqüência elemento retangular de $A$.

O próximo resultado é uma conseqüência imediata das Proposições 7.13 e 5.16.

Proposição 7.14 Seja $A$ um EE convexo. Se a seqüencia mais à esquerda de $\mathcal{T}(A)$, digamos $\left[T_{0}, T_{1}, T_{2}, \cdots, T_{j}\right]$, é maximal, então lower $(A)=j$.

Prova: Pela Proposição 7.13, $\left[T_{0}, T_{1}, \cdots, T_{j}\right]$, é uma seqüência elemento retangular de $A$. Dessa forma, pela Proposição $5.16, \operatorname{lower}\left(T_{j}\right)=j$. Como, pelo Lema $7.11, T_{j} \equiv A$, temos que, $\rho\left(T_{j}\right)=\rho(A)$, e, conseqüentemente, lower $\left(T_{j}\right)=\operatorname{lower}(A)$. Portanto, $j=$ $\operatorname{lower}\left(T_{j}\right)=\operatorname{lower}(A)$.

Teorema 7.15 Seja $A$ um EE convexo. Se a seqüência mais à esquerda de $\mathcal{T}(A)$, digamos $\left[T_{0}, T_{1}, T_{2}, \cdots, T_{j}\right]$, é maximal, então $\mathrm{Path}_{A}\left[\{o\}, T_{j}\right]$ é uma decomposição ótima de $A$.

Prova: Pelo Teorema 7.12, $\operatorname{Path}_{A}\left[\{o\}, T_{j}\right]$ é uma decomposição de $A$. Uma vez que Path $_{A}\left[\{o\}, T_{j}\right]$ contém exatamente $j$ elementos e, pela Proposição 7.14, $j=\operatorname{lower}(A)$, então $\operatorname{Path}_{A}\left[\{o\}, T_{j}\right]$ é uma decomposição ótima de $A$.

\subsubsection{Complexidade para Encontrar a Seqüência Maximal de Nós à Esquer- da}

Dado um EE convexo $A$, quando a seqüência mais à esquerda maximal de $\mathcal{T}(A)$, digamos $\left[T_{0}, T_{1}, T_{2}, \cdots, T_{j}\right]$, é encontrada, então, pelo Teorema 7.15 , Path $A\left[\{o\}, T_{j}\right]$ é uma decomposição ótima de $A$. Mostraremos que a complexidade para encontrar a seqüência mais à esquerda maximal de $\mathcal{T}(A)$ é $O\left(n^{4}\right)$, onde $n$ é o valor máximo entre as coordenadas do vetor retangular $\rho(A)$.

Seja $k$ um inteiro positivo. Sejam $A$ e $Y$ dois EE's tais que $Y$ é um nó de $\mathcal{T}(A)$ no nível $k$. Seja Path $A[\{o\}, Y]=\left[B_{1}, B_{2}, \cdots, B_{k}\right]$. Para verificar se $Y$ é um invariante de $A$, temos que comparar se $\left.A=(A \ominus Y) \oplus Y=\left(\cdots\left(\left(A \ominus B_{1}\right) \ominus B_{2} \ominus \cdots \ominus B_{j}\right) \oplus B_{1}\right) \oplus B_{2} \oplus \cdots \oplus B_{k}\right)$. A complexidade para computar $A \ominus B_{i}$ ou $A \oplus B_{i}$ é linear com relação ao número de pontos de $A$, uma vez que $B_{i}$ contém no máximo 9 pontos. Se $n$ é o valor máximo entre as coordenadas do vetor retangular $\rho(A)$, então a complexidade de tempo para computar $A \ominus B_{i}$ ou $A \oplus B_{i}$ é $O\left(n^{2}\right)$. Assim, a complexidade para verificar se um nó $Y$ no nível $k$ é invariante de $A$ é $O\left(k \cdot n^{2}\right)$.

Uma vez que o algoritmo apresentado na Seção 7.2 faz uma busca em profundidade, então a primeira seqüência de nós visitada pelo algoritmo até chegar a uma folha é a seqüência mais à esquerda maximal $\left[T_{0}, T_{1}, T_{2}, \cdots, T_{j}\right]$ de $\mathcal{T}(A)$. Se $A$ é um EE convexo, então pelo, Teorema 7.15, $\operatorname{Path}_{A}\left[\{o\}, T_{j}\right]$ é uma decomposição ótima de $A$. Assim, a 
primeira solução encontrada pelo algoritmo proposto é sempre uma decomposição ótima de $A$.

Seja $A$ um EE convexo. Pelo Teorema 4.18, $\operatorname{lower}(A)=\left\lceil\max \left\{\rho_{0}(A), \rho_{1}(A)\right\} / 2\right\rceil=$ $O(n)$, onde $n=\max \left\{\rho_{0}(A), \rho_{1}(A)\right\}$. Se a seqüência mais à esquerda de $\mathcal{T}(A)$, digamos $\left[T_{0}, T_{1}, T_{2}, \cdots, T_{j}\right]$, é maximal, então, pela Proposição $7.14, j=\operatorname{lower}(A)$. Logo, o tempo para o algoritmo encontrar a seqüência maximal mais à esquerda de $\mathcal{T}(A)$ é $O\left(1 \cdot n^{2}\right)+$ $O\left(2 \cdot n^{2}\right)+\cdots+O\left(\right.$ lower $\left.(A) \cdot n^{2}\right)$. Portanto, a complexidade para o algoritmo encontrar uma decomposição ótima de um EE convexo $A$ é $O\left(\operatorname{lower}(A)^{2} \cdot n^{2}\right)$, ou seja, $O\left(n^{4}\right)$, uma vez que lower $(A)=O(n)$.

\subsubsection{Algoritmo de Park e Chin}

Park e Chin [35] desenvolveram uma extensão do algoritmo de Xu para encontrar decomposição de EE's simplesmente conexos, onde todos elementos na decomposição são também simplesmente conexos. Vimos no Capítulo 6 que existem famílias infinitas de EE's simplesmente conexos que são decomponíveis mas não têm decomposição segundo a definição de Park e Chin.

Embora a complexidade do algoritmo proposto seja $O\left(2^{m}\right)$, onde $m$ é o número de elementos na seqüência de invariantes, ele tem uma vantagem em relação ao algoritmo de Park e Chin, uma vez que ele é capaz de decompor qualquer EE decomponível, incluindo os EE's nas famílias $\mathcal{X}, \mathcal{Y}$ e $\mathcal{Z}$ definidas no Capítulo 6 .

\subsection{Resultados Experimentais}

Nesta seção, apresentaremos alguns resultados experimentais de nosso algoritmo para encontrar uma decomposição ótima para diferentes tipos de EE's: discos euclidianos (veja definição abaixo), EE's convexos (veja um exemplo na Figura 7.5.a), EE's conexos, decomponíveis, que contém buracos (veja um exemplo na Figura 7.5.b), EE's conexos, decomponíveis, que não contém buracos (veja um exemplo na Figura 7.5.c), EE's não conexos, decomponíveis, que contém buracos (veja um exemplo na Figura 7.5.d) e EE's não conexos, decomponíveis, que não contém buracos (veja um exemplo na Figura 7.5.e). Estes experimentos foram realizados em uma máquina "Sun Ultra Enterprise 3000". O tempo de processamento da CPU está medido em horas (h), minutos (m) e segundos (s).

$\mathrm{O}$ disco euclidiano de raio $r>0$, centrado na origem, é o EE definido por $D(r)=$ $\left\{(x, y) \in \mathbb{Z}^{2}: x^{2}+y^{2} \leq r^{2}\right\}$ (veja a Figura 7.6 para alguns exemplos). Note que esta não é a única definição de EE's circulares discretos. Um método para se obter alguns tipos de EE's circulares discretos e suas respectivas decomposições pode ser encontrado em [48].

Dividiremos esta seção em três partes. Na primeira, mostramos alguns resultados para discos euclidianos; na segunda, para EE's convexos; e na terceira, para EE's decom- 


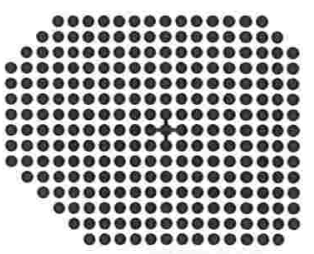

(a)

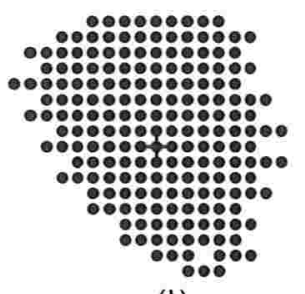

(b)

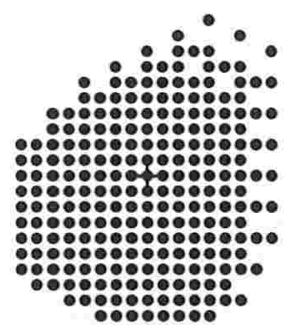

(d)

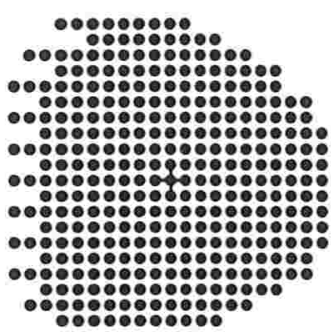

(c)

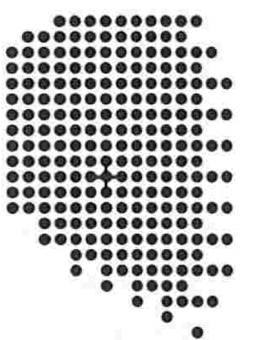

(e)

Figura 7.5: (a) Um EE convexo. (b) Um EE conexo, decomponível, que contém um buraco. (c) Um EE conexo, decomponível, que não contém buracos. (d) Um EE não conexo, decomponível, que contém alguns buracos. (e) Um EE não conexo, decomponível, que não contém buracos.

poníveis, conexos ou não, que contém buracos ou não. Nas tabelas desta seção, usaremos a seguinte notação:

NSI $=$ número de elementos na seqüência de invariantes.

NFS = número de elementos na primeira solução encontrada.

Nos $=$ número de elementos na solução ótima encontrada.

$\mathrm{TND}=$ tempo para detectar a não decomposabilidade de um EE.

TFS $=$ tempo para detectar a primeira solução.

Tos $=$ tempo para detectar a solução ótima.

\subsubsection{Discos Euclidianos}

Os discos $D(2)$ e $D(4)$ são decomponíveis, enquanto os discos $D(3)$ e $D(5)$ a $D(50)$ não têm decomposição. Os tempos para detectar a não decomposabilidade dos discos de $D(5)$ a $D(50)$ foram menores que 40 segundos. Os tempos para detectar a primeira solução 


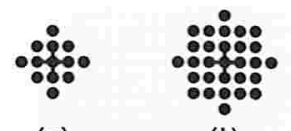

(a)

(b)

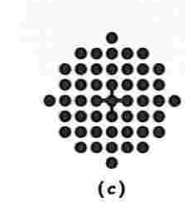

Figura 7.6: $(a)-(c)$ Discos euclidianos de raios 2, 3 e 4 .

\begin{tabular}{cccc}
\hline raio & NSI & NFS=NOS & TFS=TOS \\
\hline 2 & 5 & 2 & $0.5 \mathrm{~s}$ \\
4 & 16 & 4 & $0.7 \mathrm{~s}$ \\
\hline
\end{tabular}

Tabela 7.1: Discos decomponíveis.

(que também foi a ótima) dos discos $D(2)$ e $D(4)$ estão apresentados na Tabela 7.1. A Tabela 7.2 mostra o tempo para detectar a não decomposibilidade de alguns discos de raios entre 5 e 50 . Somente para os discos $D(15), D(20)$ e $D(25)$, o primeiro teste do algoritmo (isto é, a condição necessário para a existência de uma decomposição) foi satisfeito. Assim, isto explica o fato de que o tempo para detectar a não decomposibilidade de $D(25)$ é maior que $D(30)$.

\subsubsection{Elementos Estruturantes Convexos}

O algoritmo foi aplicado para encontrar uma decomposição ótima de aproximadamente 250 EE's convexos gerados aleatoriamente. Como foi mostrado no Teorema 7.15, toda primeira solução encontrada pelo algoritmo é uma solução ótima. A Tabela 7.3 apresenta o tempo médio para detectar a solução ótima para EE's convexos que são subconjuntos dos quadrados $20 \times 20,40 \times 40,56 \times 56,72 \times 72$ e $88 \times 88$. Por exemplo, o EE convexo apresentado na Figura 7.5. $a$ é um subconjunto do quadrado $20 \times 20$. Observamos que o tempo para encontrar a decomposição ótima de EE's convexos é muito pequeno, mesmo para EE's grandes. A Tabela 7.4 apresenta o tempo para detectar a solução ótima de alguns EE's.

A título de comparação com o algoritmo desenvolvido no Capítulo 5, mostramos na Tabela 7.5 contendo os tempos que o algoritmo proposto levou para encontrar uma decomposição ótima dos mesmos EE's indicados na Tabela 5.1. Para manter compatibilidade com o experimento apresentado no Capítulo 5, este particular experimento foi executado em uma máquina com processador Pentium III, $450 \mathrm{MHz}$.

Vemos, na Tabela 7.5, que o algoritmo DECCON é muito mais rápido do que o algoritmo proposto. Isto não é tanto devido ao fato de que a complexidade de DECCon seja 


\begin{tabular}{ccc}
\hline raio & NSI & TND \\
\hline 05 & 4 & $0.7 \mathrm{~s}$ \\
10 & 22 & $1.0 \mathrm{~s}$ \\
15 & 38 & $2.2 \mathrm{~s}$ \\
20 & 54 & $11.1 \mathrm{~s}$ \\
25 & 70 & $36.3 \mathrm{~s}$ \\
30 & 70 & $2.5 \mathrm{~s}$ \\
35 & 54 & $4.4 \mathrm{~s}$ \\
40 & 70 & $4.9 \mathrm{~s}$ \\
45 & 86 & $6.3 \mathrm{~s}$ \\
50 & 102 & $7.7 \mathrm{~s}$ \\
\hline
\end{tabular}

Tabela 7.2: Discos que não são decomponíveis.

\begin{tabular}{cc}
\hline Subconjunto do quadrado & TFS=TOS \\
\hline $20 \times 20$ & $5.5 \mathrm{~s}$ \\
$40 \times 40$ & $19.4 \mathrm{~s}$ \\
$56 \times 56$ & $47.2 \mathrm{~s}$ \\
$72 \times 72$ & $1 \mathrm{~m} 42.0 \mathrm{~s}$ \\
$88 \times 88$ & $3 \mathrm{~m} 21.0 \mathrm{~s}$ \\
\hline
\end{tabular}

Tabela 7.3: Tempo médio para se detectar a solução ótima de EE's convexos.

$O\left(n^{3}\right)$ e do algoritmo proposto seja $O\left(n^{4}\right)$, onde $n$ é o valor máximo entre as coordenadas do vetor retangular do $\mathrm{EE}$ de entrada. Este acréscimo de tempo é devido à construção da seqüência de invariantes pelo algoritmo proposto. Note que o algoritmo DECCoN não constrói a seqüência de invariantes e a seqüência SeqQ (do Passo 1) é construída previamente antes da execução do algoritmo. Para terminar esta subseção, observamos que as soluções obtidas pelo algoritmo proposto e pelo algoritmo DECCoN foram as mesmas.

\subsubsection{Elementos Estruturantes Decomponíveis, Conexos e não Conexos, com e sem Buracos}

Nesta subseção, usaremos a seguinte notação para denotar os EE's:

Dсн $=$ EE's decomponíveis e conexos que contém buracos

$\mathrm{DC}=$ EE's decomponíveis e convexos que não contém buracos.

$\mathrm{DDH}=\mathrm{EE}$ 's decomponíveis e não conexos que contém buracos.

$\mathrm{DD}=\mathrm{EE}$ 's decomponíveis e não conexos que não contém buracos.

Aplicamos o algoritmo para encontrar a primeira solução (respectivamente, solução ótima) em aproximadamente 400 (respectivamente, 200) EE's decomponíveis, conexos e não conexos, com e sem buracos. Estes EE's foram gerados aleatoriamente. Os EE's aprensetados nas Figuras de 7.5.b a 7.5.e são alguns exemplos desses EE's.

A Tabela 7.6 apresenta o tempo médio para detectar a primeira solução de EE's que são subconjuntos do quadrado $20 \times 20$ e $40 \times 40$. Por exemplo, os EE's apresentados nas 


\begin{tabular}{ccccc}
\hline EE $A$ & $\rho(A)$ & NSI & NFS=NOS & TFS=TOS \\
\hline 01 & $(17,17)$ & 448 & 9 & $5.5 \mathrm{~s}$ \\
02 & $(17,19)$ & 531 & 10 & $7.7 \mathrm{~s}$ \\
03 & $(31,34)$ & 844 & 17 & $23.3 \mathrm{~s}$ \\
04 & $(32,36)$ & 918 & 18 & $22.0 \mathrm{~s}$ \\
05 & $(48,48)$ & 1637 & 24 & $50.0 \mathrm{~s}$ \\
06 & $(52,55)$ & 1619 & 28 & $57.0 \mathrm{~s}$ \\
07 & $(65,68)$ & 2114 & 34 & $2 \mathrm{~m} 7.0 \mathrm{~s}$ \\
08 & $(69,68)$ & 2217 & 35 & $2 \mathrm{~m} 30.0 \mathrm{~s}$ \\
09 & $(85,82)$ & 2586 & 43 & $3 \mathrm{~m} 55.0 \mathrm{~s}$ \\
10 & $(88,84)$ & 2802 & 44 & $4 \mathrm{~m} 32.0 \mathrm{~s}$ \\
\hline
\end{tabular}

Tabela 7.4: Tempo para se detectar a solução ótima de alguns EE's convexos.

\begin{tabular}{|c|ccc|}
\hline & \multicolumn{3}{|c|}{$\begin{array}{c}\text { Tempo Médio para } \\
\text { encontrar uma } \\
\text { solução ótima }\end{array}$} \\
\hline $\begin{array}{c}\text { Subconjuntos do } \\
\text { retângulo }\end{array}$ & $\begin{array}{c}\text { Algoritmo } \\
\text { do Xu }\end{array}$ & $\begin{array}{c}\text { Algoritmo } \\
\text { DECCoN }\end{array}$ & $\begin{array}{c}\text { Algoritmo } \\
\text { proposto }\end{array}$ \\
\hline $20 \times 21$ & $0.22 \mathrm{~s}$ & $0.23 \mathrm{~s}$ & $1.33 \mathrm{~s}$ \\
$38 \times 40$ & $0.23 \mathrm{~s}$ & $0.27 \mathrm{~s}$ & $3.25 \mathrm{~s}$ \\
$56 \times 57$ & $0.23 \mathrm{~s}$ & $0.26 \mathrm{~s}$ & $8.16 \mathrm{~s}$ \\
$73 \times 73$ & $0.26 \mathrm{~s}$ & $0.28 \mathrm{~s}$ & $17.48 \mathrm{~s}$ \\
$89 \times 89$ & $0.28 \mathrm{~s}$ & $0.28 \mathrm{~s}$ & $31.70 \mathrm{~s}$ \\
$109 \times 108$ & $0.25 \mathrm{~s}$ & $0.33 \mathrm{~s}$ & $67.95 \mathrm{~s}$ \\
$124 \times 124$ & $0.32 \mathrm{~s}$ & $0.54 \mathrm{~s}$ & $2 \mathrm{~m} 1.09 \mathrm{~s}$ \\
$141 \times 144$ & $0.29 \mathrm{~s}$ & $0.42 \mathrm{~s}$ & $3 \mathrm{~m} 15.36 \mathrm{~s}$ \\
$160 \times 162$ & $0.31 \mathrm{~s}$ & $0.47 \mathrm{~s}$ & $6 \mathrm{~m} 0.87 \mathrm{~s}$ \\
$176 \times 179$ & $0.35 \mathrm{~s}$ & $0.56 \mathrm{~s}$ & $8 \mathrm{~m} 17.34 \mathrm{~s}$ \\
$192 \times 193$ & $0.37 \mathrm{~s}$ & $0.63 \mathrm{~s}$ & $11 \mathrm{~m} 20.19 \mathrm{~s}$ \\
$214 \times 210$ & $0.41 \mathrm{~s}$ & $0.75 \mathrm{~s}$ & $18 \mathrm{~m} 20.30 \mathrm{~s}$ \\
$229 \times 227$ & $0.45 \mathrm{~s}$ & $0.86 \mathrm{~s}$ & $24 \mathrm{~m} 23.05 \mathrm{~s}$ \\
$246 \times 247$ & $0.48 \mathrm{~s}$ & $0.97 \mathrm{~s}$ & $34 \mathrm{~m} 30.58 \mathrm{~s}$ \\
\hline
\end{tabular}

Tabela 7.5: Tempo médio para encontrar uma decomposição ótima de EE's convexos.

Figuras de 7.5.b a 7.5.e são subconjuntos do quadrado $20 \times 20$. Na maioria dos casos, a primeira solução encontrada foi também a solução ótima. Além disso, a diferença entre o número de elementos na primeira solução e o limite inferior de uma decomposição foi no máximo dois. Portanto, na maioria das vezes, a primeira solução esteve muito perto da solução ótima.

Na Tabela 7.7, apresentamos o tempo para detectar a primeira solução e a solução ótima de alguns EE's que são subconjuntos do quadrado $20 \times 20$ e que o limite inferior não foi igual ao número de elementos na primeira solução. Nesta tabela, observe que a diferença entre o número de elementos na primeira solução e o limite inferior é normalmente pequeno e, na maioria dos casos, a primeira solução é a solução ótima. Uma vez que a complexidade de tempo para detectar a solução ótima, no pior caso, é exponencial, em aplicações práticas, uma boa heurística é parar quando a primeira solução é encontrada.

Embora os resultados apresentados nas Tabelas 7.6 e 7.7 sejam bons, o tempo para encontrar a primeira solução aumentou exponencialmente com o acréscimo do tamanho do EE. Isto aconteceu pois as três estratégias de podas usadas no algoritmo não foram 


\begin{tabular}{lcc}
\hline & $20 \times 20$ & $40 \times 40$ \\
\hline DCH & $3.266 \mathrm{~s}$ & $13 \mathrm{~m} 41.0 \mathrm{~s}$ \\
DC & $2.876 \mathrm{~s}$ & $22.2 \mathrm{~s}$ \\
DDH & $1.682 \mathrm{~s}$ & $34.0 \mathrm{~s}$ \\
DD & $1.506 \mathrm{~s}$ & $18 \mathrm{~m} 27.0 \mathrm{~s}$ \\
\hline
\end{tabular}

Tabela 7.6: Tempo médio para detectar a primeira solução de alguns EE's.

\begin{tabular}{|c|c|c|c|c|c|c|c|c|}
\hline EE $A$ & $\rho(A)$ & NSI & limite & inferior & NFS & TFS & NOS & TOS \\
\hline \multirow{5}{*}{$\mathrm{DDH}$} & $(18,16)$ & 17 & & 9 & 10 & $1.2 \mathrm{~s}$ & 10 & $3.0 \mathrm{~s}$ \\
\hline & $(16,16)$ & 17 & & 8 & 9 & $1.0 \mathrm{~s}$ & 9 & $4.1 \mathrm{~s}$ \\
\hline & $(18,18)$ & 38 & & 9 & 10 & $1.8 \mathrm{~s}$ & 10 & $29.4 \mathrm{~s}$ \\
\hline & $(18,15)$ & 21 & & 9 & 10 & $1.1 \mathrm{~s}$ & 10 & $6.0 \mathrm{~s}$ \\
\hline & $(14,18)$ & 10 & & 9 & 10 & $1.0 \mathrm{~s}$ & 10 & $1.3 \mathrm{~s}$ \\
\hline \multirow{4}{*}{ DD } & $(16,16)$ & 19 & & 8 & 9 & $1.1 \mathrm{~s}$ & 9 & $7.5 \mathrm{~s}$ \\
\hline & $(18,17)$ & 29 & & 9 & 10 & $1.2 \mathrm{~s}$ & 10 & $43.5 \mathrm{~s}$ \\
\hline & $(18,14)$ & 36 & & 9 & 10 & $1.5 \mathrm{~s}$ & 10 & $1 \mathrm{~m} 54.0 \mathrm{~s}$ \\
\hline & $(18,16)$ & 20 & & 9 & 10 & $1.3 \mathrm{~s}$ & 10 & $7.7 \mathrm{~s}$ \\
\hline \multirow{5}{*}{ DCH } & $(18,16)$ & 24 & & 9 & 10 & $1.2 \mathrm{~s}$ & 10 & $11.6 \mathrm{~s}$ \\
\hline & $(16,16)$ & 41 & & 8 & 9 & $3.5 \mathrm{~s}$ & 9 & $2 \mathrm{~m} 39.0 \mathrm{~s}$ \\
\hline & $(18,18)$ & 30 & & 9 & 10 & $1.2 \mathrm{~s}$ & 10 & $57.2 \mathrm{~s}$ \\
\hline & $(16,18)$ & 19 & & 9 & 10 & $1.0 \mathrm{~s}$ & 10 & $2.5 \mathrm{~s}$ \\
\hline & $(16,18)$ & 30 & & 9 & 10 & $1.8 \mathrm{~s}$ & 10 & $18.0 \mathrm{~s}$ \\
\hline \multirow{4}{*}{ DC } & $(16,17)$ & 60 & & 9 & 10 & $4.1 \mathrm{~s}$ & 9 & $47.0 \mathrm{~s}$ \\
\hline & $(14,18)$ & 43 & & 9 & 10 & $5.3 \mathrm{~s}$ & 10 & $2 \mathrm{~m} 41.0 \mathrm{~s}$ \\
\hline & $(16,15)$ & 31 & & 8 & 9 & $1.6 \mathrm{~s}$ & 9 & $27.0 \mathrm{~s}$ \\
\hline & $(18,18)$ & 41 & & 9 & 10 & $1.6 \mathrm{~s}$ & 10 & $8 \mathrm{~m} 20.3 \mathrm{~s}$ \\
\hline
\end{tabular}

Tabela 7.7: Tempo para detectar a primeira solução e a solução ótima de alguns EE's.

capazes de evitar um número considerável de nós não viáveis na árvore de decomposição. A Tabela 7.8 apresenta o tempo para detectar a primeira solução de alguns EE's que são maiores que os EE's apresentados na Tabela 7.7 .

\subsection{Conclusão}

A mudança ou transformação de estrutura de representação de operadores para melhorar o desempelho de suas implementações é um passo fundamental no processo de programação automática de MaqM's [11]. Neste capítulo, estudamos um aspecto particular deste problema: a decomposição seqüencial de erosões (respectivamente, dilatações).

Um algoritmo geral para prova automática de que uma erosão (respectivamente, dilatação) tenha uma decomposição seqüencial ou não foi apresentado. A prova da existência é construtiva e uma solução ótima é exibida. Este algoritmo é baseado em uma busca que usa o método "branch and bound", com estratégias de podas baseadas em propriedades geométricas e algébricas deduzidas formalmente.

O algoritmo proposto não é eficiente para todos os casos, mas generaliza importantes resultados clássicos (Zhuang and Haralick, Xu, Park e Chin). Uma análise teórica do algoritmo, juntamente com alguns resultados experimentais, ilustra estes fatos. O algoritmo 


\begin{tabular}{c|ccc}
\hline EE $A$ & $\rho(A)$ & NSI & TFS \\
\hline DDH & $(62,75)$ & 114 & $67 \mathrm{~h} 38 \mathrm{~m} 56.0 \mathrm{~s}$ \\
\hline DD & $(52,56)$ & 91 & 5h25m30.0s \\
\hline DCH & $(50,54)$ & 137 & 23h24m21.0s \\
\hline DC & $(50,58)$ & 521 & Pelo menos 201h \\
\hline
\end{tabular}

Tabela 7.8: Tempo para detectar a primeira solução de alguns EE's.

combinatorial apresentado pode ser melhorado se novos limites ou estratégias de podas forem descobertos.

\subsection{Apêndice do Capítulo 7}

Neste apêndice, vamos provar a Proposição 7.13. Dividiremos esta prova em duas partes. Na primeira, mostraremos alguns resultados intermediários necessários para a prova e, na segunda parte, provaremos a Proposição 7.13.

\subsubsection{Resultados Necessários para a Prova da Proposição 7.13}

Dado um EE $A$, para mostrar que uma seqüência maximal de nós mais à esquerda de $\mathcal{T}(A)$, digamos $\left[T_{0}, T_{1}, \cdots, T_{j}\right]$, é uma seqüência elemento retangular de $A$, precisamos de alguns resultados preliminares, dados nos Lemas de 7.16 a 7.18.

Lema 7.16 Seja $A$ um EE convexo. Seja $\left[T_{0}, T_{1}, \cdots, T_{j}\right]$ uma seqüência de nós mais à esquerda de $\mathcal{T}(A)$. Seja Path $_{A}\left[\{o\}, T_{j}\right]=\left[D_{1}, D_{2}, \cdots, D_{j}\right]$ e seja $B$ um elemento de Seqlnv $[A]$. Se, para algum $k \in\{1,2, \cdots, j\}, T_{k-1} \oplus B$ é um invariante de $A$, então $\rho_{0}\left(D_{k}\right)+\rho_{1}\left(D_{k}\right) \geq \rho_{0}(B)+\rho_{1}(B)$.

Prova: Uma vez que $B$ é um elemento de $\operatorname{Seq} \operatorname{lnv}[A]$, a seqüência $\left[T_{0}, T_{1}, \cdots, T_{k-1}\right]$ é uma seqüência de nós mais à esquerda de $\mathcal{T}(A)$ e $T_{k-1} \oplus B$ é um invariante de $A$, então, pelo Lema $7.9, B$ é um elemento de $\operatorname{Arestas}_{A}\left[T_{k-1}\right]$. Como $D_{k}$ é o primeiro elemento de $\operatorname{Arestas}_{A}\left[T_{k-1}\right]$ tal que $T_{k-1} \oplus D_{k}$ é invariante de $A$, então $B$ aparece depois de $D_{k}$ em Arestas $_{A}\left[T_{k-1}\right]$. Assim, pela ordem escolhida para construir a seqüência Seqlnv $[A]$, $\rho_{0}\left(D_{k}\right)+\rho_{1}\left(D_{k}\right) \geq \rho_{0}(B)+\rho_{1}(B)$.

Lema 7.17 Seja $A$ um EE convexo. Seja $\left[D_{1}, D_{2}, \cdots, D_{j}\right]$ uma subseqüência de Seqlnv[A] com $j \geq 2$ tal que $D_{1} \oplus D_{2} \oplus \cdots \oplus D_{j}$ é um invariante de $A$. Sejam $D_{i}$ e $D_{k}$ dois elementos de $\left[D_{1}, D_{2}, \cdots, D_{j}\right]$ tais que $i<k$. Se $T$ é um EE tal que $|T| \geq 2, \rho(T) \leq(2,2)$ e $T$ é um invariante de $D_{i} \oplus D_{k}$ ou $C\left(D_{i}\right) \oplus C\left(D_{k}\right)$, então existe um $B$ em Seqlnv $[A]$ tal que $\rho(B)=\rho(T)$ e $D_{1} \oplus D_{2} \oplus \cdots \oplus D_{i-1} \oplus B$ é im invariante de $A$. 
Prova: Seja $X=D_{1} \oplus D_{2} \oplus \cdots \oplus D_{i-1}$. Seja $Y$ o resultado da adição de Minkowski de todos elementos em $\left[D_{i}, D_{i+1}, \cdots, D_{j}\right]$ com exceção de $D_{i}$ e $D_{k}$. Assim, $Z=D_{1} \oplus D_{2} \oplus$ $\cdots \oplus D_{j}=Y \oplus X \oplus D_{i} \oplus D_{k}$.

Uma vez que $Z=D_{1} \oplus D_{2} \oplus \cdots \oplus D_{j}$ é invariante de $A$, pela Proposição 3.14, existe um EE $R$ tal que $A=R \oplus Z=R \oplus\left(Y \oplus X \oplus D_{i} \oplus D_{k}\right)=(R \oplus Y) \oplus\left(X \oplus D_{i} \oplus D_{k}\right)=$ $S \oplus\left(X \oplus D_{i} \oplus D_{k}\right)$, onde $S=R \oplus Y$. Dessa forma, pela Proposição $3.15, D_{i} \oplus D_{k}$ é um invariante de $A$.

Como $A$ é um EE convexo e $A=S \oplus X \oplus D_{i} \oplus D_{k}$, então, pela Proposição 5.4, $A=S \oplus X \oplus C\left(D_{i}\right) \oplus D_{k}=S \oplus X \oplus C\left(D_{i}\right) \oplus C\left(D_{k}\right)$. Dessa forma, pela Proposição 3.15, $C\left(D_{i}\right) \oplus C\left(D_{k}\right)$ é um invariante de $A$.

Uma vez que $T$ é um invariante de $D_{i} \oplus D_{k}$ ou $C\left(D_{i}\right) \oplus C\left(D_{k}\right)$; e $D_{i} \oplus D_{k}$ e $C\left(D_{i}\right) \oplus C\left(D_{k}\right)$ são invariantes de $A$, então, pela Proposição 5.2, $T$ é um invariante de $A$.

Como que $\rho(T) \leq(2,2)$ e $T$ é um invariante de $A$, então existe um elemento $B$ em Seqlnv $[A]$ tal que $B \equiv T$. Claramente, $\rho(B)=\rho(T)$. Agora, falta demonstrar que $X \oplus B$ é um invariante de $A$.

Uma vez que $T$ é um invariante de $D_{i} \oplus D_{k}$ ou $C\left(D_{i}\right) \oplus C\left(D_{k}\right)$, então, claramente, $B$ também é um invariante de $D_{i} \oplus D_{k}$ ou $C\left(D_{i}\right) \oplus C\left(D_{k}\right)$.

Se $B$ é um invariante de $D_{i} \oplus D_{k}$, então, pela Proposição 3.14, existe um EE $D$ tal que $B \oplus D=D_{i} \oplus D_{k}$. Assim, como $A=S \oplus X \oplus D_{i} \oplus D_{k}=S \oplus X \oplus\left(D_{i} \oplus D_{k}\right)=$ $S \oplus(X \oplus B \oplus D)=S \oplus(X \oplus B) \oplus D$, então, pela Proposição 3.15, $X \oplus B$ é um invariante de $A$.

Analogamente, se $B$ é um invariante de $C\left(D_{i}\right) \oplus C\left(D_{k}\right)$, então $X \oplus B$ é um invariante de $A$.

Lema 7.18 Seja $A$ um EE convexo. Seja $\left[D_{1}, D_{2}, \cdots, D_{j}\right]$ uma subseqüência de Seqlnv $[A]$ com $j \geq 2$ tal que $D_{1} \oplus D_{2} \oplus \cdots \oplus D_{j}$ é um invariante de $A$. Se existem $D_{i}$ e $D_{k}$ em $\left[D_{1}, D_{2}, \cdots, D_{j}\right]$ tais que $\rho\left(D_{i}\right)=(2,1)$ e $\rho\left(D_{k}\right)=(1,2)$, então existe $B$ em Seqlnv $[A]$ tal que $\rho(B)=(2,2)$ e $D_{1} \oplus D_{2} \oplus \cdots \oplus D_{\ell-1} \oplus B$ é um invariante de $A$, onde $\ell=\min \{i, k\}$.

Prova: Sejam $\mathcal{R}, \mathcal{R}_{1}, \mathcal{R}_{2}, \mathcal{S}, \mathcal{S}_{1}$ e $\mathcal{S}_{2}$ os conjuntos definidos na Proposição 5.24, cujos elementos estão mostrados nas Figuras 5.3 e 5.4. Da mesma forma que foi feito na Proposição 5.24, suponha que $C\left(D_{i}\right) \in \mathcal{R}$ e $C\left(D_{k}\right) \in \mathcal{S}$. Assim, uma vez que $\left\{\mathcal{R}_{1}, \mathcal{R}_{2}\right\}$ e $\left\{\mathcal{S}_{1}, \mathcal{S}_{2}\right\}$ são partições, respectivamente, de $\mathcal{R}$ e $\mathcal{S}$, temos dois casos para considerar: (1) $C\left(D_{i}\right) \in \mathcal{R}_{1}$ ou $C\left(D_{k}\right) \in \mathcal{S}_{1}$; e (2) $C\left(D_{i}\right) \in \mathcal{R}_{2}$ e $C\left(D_{i}\right) \in \mathcal{S}_{2}$.

Caso 1: $C\left(D_{i}\right) \in \mathcal{R}_{1}$ ou $C\left(D_{k}\right) \in \mathcal{S}_{1}$.

Consideraremos que $C\left(D_{i}\right) \in \mathcal{R}_{1}$ (a prova para $C\left(D_{k}\right) \in \mathcal{S}_{1}$ pode ser feita de maneira similar). 
Como indicado na Figura 5.5, se $C\left(D_{i}\right) \in \mathcal{R}_{1}$, então existem dois EE's convexos $C_{1}$ e $C_{2}$ tais que $C\left(D_{i}\right)=C_{1} \oplus C_{2}, \rho\left(C_{1}\right)=(1,1)$ e $\rho\left(C_{2}\right)=(1,0)$. Dessa forma, $C\left(D_{i}\right) \oplus C\left(D_{k}\right)=\left(C_{1} \oplus C_{2}\right) \oplus C\left(D_{k}\right)=C_{1} \oplus\left(C_{2} \oplus C\left(D_{k}\right)\right)=C_{1} \oplus T$, onde $T=C_{2} \oplus C\left(D_{k}\right)$. Assim, uma vez que $T$ é um invariante de $C\left(D_{i}\right) \oplus C\left(D_{k}\right)$ (pela Proposição 3.15) e $\rho(T)=\rho\left(C_{2}\right)+\rho\left(C\left(D_{k}\right)\right)=(2,2)$ (pela Proposição 4.17), então, pelo Lema 7.17, existe um elemento $B$ em Seqlnv $[A]$ tal que $\rho(B)=\rho(T)=(2,2) \mathrm{e}$ $D_{1} \oplus D_{2} \oplus \cdots \oplus D_{i-1} \oplus B$ é um invariante de $A$ (se $i<k$ ) ou $D_{1} \oplus D_{2} \oplus \cdots \oplus D_{k-1} \oplus B$ é um invariante de $A$ (se $k<i$ ).

Caso 2: $\quad C\left(D_{i}\right) \in \mathcal{R}_{2}$ e $C\left(D_{i}\right) \in \mathcal{S}_{2}$.

Neste caso, a Figura 5.7 apresenta todas possibilidades de $C\left(D_{i}\right) \oplus C\left(D_{k}\right)$. Assim, como indicado nesta figura, existem dois EE's convexos $C_{1}$ e $C_{2}$ tais que $C\left(D_{i}\right) \oplus$ $C\left(D_{k}\right)=C_{1} \oplus C_{2}, \rho\left(C_{1}\right)=(2,2)$ e $\rho\left(C_{2}\right)=(1,1)$. Assim, pela Proposição 3.15 , $C_{1}$ é um invariante de $C\left(D_{i}\right) \oplus C\left(D_{k}\right)$. Dessa forma, pelo Lema 7.17, existe $B$ em Seqlnv $[A]$ tal que $\rho(B)=\rho\left(C_{1}\right)=(2,2)$ e $D_{1} \oplus D_{2} \oplus \cdots \oplus D_{i-1} \oplus B$ é um invariante de $A$ (se $i<k$ ) ou $D_{1} \oplus D_{2} \oplus \cdots \oplus D_{k-1} \oplus B$ é um invariante de $A$ (se $k<i$ ).

Portanto, existe $B$ em Seqlnv $[A]$ tal que $\rho(B)=(2,2)$ e $D_{1} \oplus D_{2} \oplus \cdots \oplus D_{\ell-1} \oplus B$ é im invariante de $A$, onde $\ell=\min \{i, k\}$.

Agora, com estes resultados dados acima, podemos mostrar que, dado um EE convexo $A$, se $\left[T_{0}, T_{1}, \cdots, T_{j}\right]$ é uma seqüência maximal de nós mais à esquerda de $\mathcal{T}(A)$, então $\left[T_{0}, T_{1}, \cdots, T_{j}\right]$ é uma seqüência elemento retangular de $A$.

\subsubsection{Prova da Proposição 7.13}

Seja Path $\operatorname{Pa}_{A}\left[\{o\}, T_{j}\right]=\left[D_{1}, D_{2}, \cdots, D_{j}\right]$. Obviamente, por construção, $\operatorname{Path}_{A}\left[\{o\}, T_{j}\right]$ é a seqüência geradora de $\left[T_{0}, T_{1}, \cdots, T_{j}\right]$. Assim, para provar que a seqüência $\left[T_{0}, T_{1}, \cdots, T_{j}\right]$ é uma seqüência elemento retangular de $A$, basta mostrar que sua seqüência geradora $\operatorname{Path}_{A}\left[\{o\}, T_{j}\right]$ é uma seqüência retangular. Dessa forma, temos que provar que as Restrições da definição de seqüência retangular são válidas para a seqüência $\operatorname{Path}_{A}\left[\{o\}, T_{j}\right]$.

Prova que as Restrições de $(a)$ a $(f)$ são válidas:

Suponha, por absurdo, que uma das Restrições de $(a)$ a $(f)$ da definição de seqüência retangular não seja válida para a seqüência $\operatorname{Path}_{A}\left[\{o\}, T_{j}\right]$. Assim, existem dois elementos $D_{i}$ e $D_{k}$, com $i<k$, na seqüência Path Pa $_{A}\left[\{o\}, T_{j}\right]=\left[D_{1}, D_{2}, \cdots, D_{j}\right]$ tais que $\rho\left(D_{i} \oplus D_{k}\right)=$ $\rho\left(D_{i}\right)+\rho\left(D_{k}\right) \leq(2,2)$. Seja $T=D_{i} \oplus D_{k}$. Claramente, $T$ é um invariante de $D_{i} \oplus D_{k}$. Além disso, como $D_{i}$ e $D_{k}$ são elementos de Seqlnv $[A]$, então $|T| \geq 2$ e $\rho_{0}\left(D_{k}\right)+\rho_{1}\left(D_{k}\right)>0$.

Uma vez que $\rho(T)=\rho\left(D_{i}\right)+\rho\left(D_{k}\right) \leq(2,2),|T| \geq 2$ e $T$ é um invariante de $D_{i} \oplus D_{k}$, então, pelo Lema 7.17, existe um elemento $B$ em Seqlnv $[A]$ tal que $\rho(B)=\rho(T)$ e $D_{1} \oplus$ $D_{2} \oplus \cdots \oplus D_{i-1} \oplus B=T_{i-1} \oplus B$ é invariante de $A$. Como $i \in\{1,2, \cdots, j\}$, então, pelo Lema 7.16, $\rho_{0}\left(D_{i}\right)+\rho_{1}\left(D_{i}\right)>\rho_{0}(B)+\rho_{1}(B)$. 
Uma vez que $\rho(B)=\rho(T)=\rho\left(D_{i}\right)+\rho\left(D_{k}\right)$, então

$$
\begin{aligned}
\rho_{0}\left(D_{i}\right)+\rho_{1}\left(D_{i}\right) \geq \rho_{0}(B)+\rho_{1}(B) & =\left(\rho_{0}\left(D_{i}\right)+\rho_{0}\left(D_{k}\right)\right)+\left(\rho_{1}\left(D_{i}\right)+\rho_{1}\left(D_{k}\right)\right) \\
& =\left(\rho_{0}\left(D_{i}\right)+\rho_{1}\left(D_{i}\right)\right)+\left(\rho_{0}\left(D_{k}\right)+\rho_{1}\left(D_{k}\right)\right) .
\end{aligned}
$$

Dessa forma, $\rho_{0}\left(D_{k}\right)+\rho_{1}\left(D_{k}\right) \leq 0$. Mas isto é um contradição, pois $\rho_{0}\left(D_{k}\right)+\rho_{1}\left(D_{k}\right)>0$. Portanto, todas as Restrições de $(a)$ a $(f)$, são válidas para $\operatorname{Path}_{A}\left[\{o\}, T_{j}\right]$.

Prova que as Restrições de $(g)$ a $(j)$ são válidas:

Suponha por absurdo que uma das restrições, de $(g)$ a $(j)$, não seja válida. Dessa forma, $c_{2,0}\left(\operatorname{Path}_{A}\left[\{o\}, T_{j}\right]\right)>0$ ou $c_{0,2}\left(\operatorname{Path}_{A}\left[\{o\}, T_{j}\right]\right)>0$. Conseqüentemente, existe um elemento $D_{k}$ em $\operatorname{Path}_{A}\left[\{o\}, T_{j}\right]$ tal que $\rho\left(D_{k}\right)=(2,0)$ ou $\rho\left(D_{k}\right)=(0,2)$. Consideraremos o caso $\rho\left(D_{k}\right)=(2,0)$. O outro caso $\rho\left(D_{k}\right)=(0,2)$ pode ser provado de uma maneira similar. Logo, pelas restrições $(g)$ ou $(h), c_{1,1}\left(\operatorname{Path}_{A}\left[\{o\}, T_{j}\right]\right)>0$ ou $c_{1,2}\left(\operatorname{Path}_{A}\left[\{o\}, T_{j}\right]\right)>0$, e, portanto, existe um elemento $D_{i}$ em Path $_{A}\left[\{o\}, T_{j}\right]$ tal que $\rho\left(D_{i}\right)=(1,1)$ ou $\rho\left(D_{i}\right)=(1,2)$. Uma vez que $\rho_{0}\left(D_{i}\right)+\rho_{1}\left(D_{i}\right) \geq \rho_{0}\left(D_{k}\right)+\rho_{1}\left(D_{k}\right)$, pela ordem escolhida para construir Seqlnv $[A]$, podemos considerar que $i<k$ (o caso $i>k$ é analógo).

Uma vez que, $\rho\left(C\left(D_{k}\right)\right)=\rho\left(D_{k}\right)=(2,0)$ (pela definição de vetor retangular), então $C\left(D_{k}\right)$ é uma linha horizontal com três pontos consecutivos. Dessa forma, existem duas linhas horizontais com dois pontos consecutivos cada um, digamos $L_{1}$ e $L_{2}$, tais que $C\left(D_{k}\right)=L_{1} \oplus L_{2}$. Claramente, $\rho\left(L_{1}\right)=\rho\left(L_{2}\right)=(1,0)$.

Assim, $C\left(D_{i}\right) \oplus C\left(D_{k}\right)=C\left(D_{i}\right) \oplus\left(L_{1} \oplus L_{2}\right)=\left(C\left(D_{i}\right) \oplus L_{1}\right) \oplus L_{2}$. Dessa forma, pela Proposição 3.15, $T=C\left(D_{i}\right) \oplus L_{1}$ é um invariante de $C\left(D_{i}\right) \oplus C\left(D_{k}\right)$. Além disso, pela Proposição 4.17, $\rho(T)=\rho\left(C\left(D_{i}\right)\right)+\rho\left(L_{1}\right) \leq(2,2)$, uma vez que $\rho\left(D_{i}\right)=(1,1)$ ou $\rho\left(D_{i}\right)=(1,2)$.

Uma vez que, $\rho(T) \leq(2,2), B$ é um invariante de $C\left(D_{i}\right) \oplus C\left(D_{k}\right)$ e $i<k$, então, pelo Lema 7.17, existe um elemento $B$ em Seqlnv[A] tal que $\rho(B)=\rho(T)$ e $D_{1} \oplus D_{2} \oplus \cdots \oplus$ $D_{i-1} \oplus B=T_{i-1} \oplus B$ é invariante de $A$. Dessa forma, uma vez que $i \in\{1,2, \cdots, j\}$, então, pelo Lema 7.16, $\rho_{0}\left(D_{i}\right)+\rho_{1}\left(D_{i}\right)>\rho_{0}(B)+\rho_{1}(B)$.

Uma vez que $\rho(B)=\rho(T)=\rho\left(C\left(D_{i}\right)\right)+\rho\left(L_{1}\right)$ e $\rho\left(C\left(D_{i}\right)\right)=\rho\left(D_{i}\right)$, então

$$
\begin{aligned}
\rho_{0}\left(D_{i}\right)+\rho_{1}\left(D_{i}\right) \geq \rho_{0}(B)+\rho_{1}(B) & =\left(\rho_{0}\left(C\left(D_{i}\right)\right)+\rho_{0}\left(L_{1}\right)\right)+\left(\rho_{1}\left(C\left(D_{i}\right)\right)+\rho_{1}\left(L_{1}\right)\right) \\
& =\left(\rho_{0}\left(D_{i}\right)+\rho_{0}\left(L_{1}\right)\right)+\left(\rho_{1}\left(D_{i}\right)+\rho_{1}\left(L_{1}\right)\right) \\
& =\left(\rho_{0}\left(D_{i}\right)+\rho_{1}\left(D_{i}\right)\right)+\left(\rho_{0}\left(L_{1}\right)+\rho_{1}\left(L_{1}\right)\right) .
\end{aligned}
$$

Assim, $\rho_{0}\left(L_{1}\right)+\rho_{1}\left(L_{1}\right) \leq 0$. Mas isto contradiz o fato de que $\rho\left(L_{1}\right)=(1,0)$. Logo, as Restrições de $(g)$ a $(j)$ são válidas para $\operatorname{Path}_{A}\left[\{o\}, T_{j}\right]$.

Prova que a Restrição $(k)$ é válida:

Suponha por absurdo que a Restrição $(k)$ não seja válida, ou seja, $c_{1,2}\left(\operatorname{Path}_{A}\left[\{o\}, T_{j}\right]\right)>$ 0 e $c_{2,1}\left(\right.$ Path $\left._{A}\left[\{o\}, T_{j}\right]\right)>0$. Sejam $D_{i}$ e $D_{k}$ dois elementos em Path ${ }_{A}\left[\{o\}, T_{j}\right]$ tais que, $\rho\left(D_{i}\right)=(1,2)$ e $\rho\left(D_{k}\right)=(2,1)$. Sem perda de generalidade suponha que $i<k$.

Pelo Lema 7.18, existe $B$ em Seqlnv $[A]$ tal que $\rho(B)=(2,2)$ e $D_{1} \oplus D_{2} \oplus \cdots \oplus D_{i-1} \oplus B=$ 
$T_{i-1} \oplus B$ é um invariante de $A$. Dessa forma, uma vez que $i \in\{1,2, \cdots, j\}$, pelo Lema 7.16, $\rho_{0}\left(D_{i}\right)+\rho_{1}\left(D_{i}\right)>\rho_{0}(B)+\rho_{1}(B)$. Mas, isto contradiz o fato de que $\rho_{0}\left(D_{i}\right)+\rho_{1}\left(D_{i}\right)=3 \mathrm{e}$ $\rho_{0}(B)+\rho_{1}(B)=4$. Logo, $c_{1,2}\left(\operatorname{Path}_{A}\left[\{o\}, T_{j}\right]\right)=0$ ou $c_{2,1}\left(\operatorname{Path}_{A}\left[\{o\}, T_{j}\right]\right)=0$ e, portanto, $c_{2,1}\left(\operatorname{Path}_{A}\left[\{o\}, T_{j}\right]\right) \cdot c_{1,2}\left(\operatorname{Path}_{A}\left[\{o\}, T_{j}\right]\right)=0$. 


\section{Capítulo 8}

\section{Representação Compacta de $W$-Operadores}

A importância dos operadores da família dos $W$-operadores na área de Visão Computacional é que eles são freqüentemente usados para resolver problemas de análise de imagens [41].

Vimos no Capítulo 3, nos Teoremas 3.35 e 3.36, (veja na página 30), que qualquer $W$ operador pode ser representado em termos de sup-geradores e inf-geradores. Neste mesmo capítulo, na Seção 3.5.3, apresentamos uma outra representação de um $W$-operador, que chamamos de sup-representação (respectivamente, inf-representação), dado pelo Teorema 3.38 (respectivamente, Teorema 3.39), que usa um número menor de sup-geradores (respectivamente, inf-geradores) que a representação canônica.

Neste capítulo, apresentamos uma nova sup-representação (respectivamente, inf-representação) para $W$-operadores que usa composições de sup-geradores (respectivamente, infgeradores) com dilatações (respectivamente, erosões) como outra família de sup-geradores (respectivamente, inf-geradores). Esta representação é chamada compacta, uma vez que ela usa um número menor (ou, no pior caso, o mesmo número) de operadores supgeradores (respectivamente, inf-geradores) que a sup-representação (respectivamente, infrepresentação) apresentada no Teorema 3.38 (respectivamente, Teorema 3.39).

Considerando os $W$-operadores que são anti-extensivos e idempotentes em um sentido estrito, nós obtivemos uma simplificação para a sua representação compacta. Além disso, colocando a hipótese de que os $W$-operadores são também crescentes, obtivemos uma realização minimal da representação clássica de Matheron para as aberturas e fechamentos IT's (como supremo de aberturas morfológicas ou ínfimo de fechamentos morfológicos) a partir da representação compacta simplificada.

Um corolário desses resultados é que a sup-representação pode ser convertida para a representação compacta por algoritmos simples e não sofisticados. Este método é útil, por exemplo, para otimizar programas da MaqM projetados por algoritmos de aprendizagem, 
uma vez que esses programas gerados automaticamente estão freqüentemente na forma de sup-representação e a representação compacta tem uma eficiência maior, pois ela usa um número menor de operadores sup-geradores que a sup-representação.

Na Seção 8.1 apresentamos a sup-representação e a inf-representação compacta dos $W$-operadores. Na Seção 8.2, mostramos uma especialização da sup-representação e da inf-representação compacta para operadores que não são crescentes. Na Seção 8.3, deduzimos a realização minimal da representação de Matheron para aberturas e fechamentos IT's a partir da representação compacta. Na Seção 8.4, apresentamos alguns exemplos de conversões da sup-representação para a sup-representação compacta. Finalmente, na Seção 8.5, fornecemos algumas conclusões deste capítulo.

\subsection{Representação Compacta}

Nesta seção, apresentaremos uma nova estrutura de decomposição para $W$-operadores que simplifica a sup-representação, no sentido de que o número de operadores sup-geradores envolvidos nesta nova decomposição é menor, ou no pior caso, igual ao número dos supgeradores na sup-representação. Por causa dessa propriedade, chamaremos essas novas decomposições de representação compacta.

Introduziremos agora uma relação de equivalência sobre uma coleção genérica de intervalos maximais $\mathbf{X}$. Sejam $[A, B]$ e $\left[A^{\prime}, B^{\prime}\right]$ dois elementos de $\mathbf{X}$. Dizemos que $[A, B]$ e $\left[A^{\prime}, B^{\prime}\right]$ são equivalentes sob translação se, e somente se, um pode ser construído a partir de uma translação do outro, isto é, $[A, B] \equiv\left[A^{\prime}, B^{\prime}\right]$ se, e somente se, existe $h \in E$ tal que $[A, B]=\left[A^{\prime}, B^{\prime}\right]_{h}$.

Uma vez que a equivalência sob translação definida acima é uma relação de equivalência, o conjunto de suas classes de equivalência (isto é, os conjuntos compostos exatamente por todos elementos equivalentes em $\mathrm{X}$ ) constitui uma partição de $\mathrm{X}$.

Denotaremos por $\mathbf{Q}(\mathbf{B}(\psi))$ o conjunto de todas as classes de equivalências (sob translação) sobre $\mathbf{B}(\psi)$, onde $\psi$ é um $W$-operador. Denotaremos por $\mathbf{E}(\psi)=\mathbf{R}(\mathbf{B}(\psi))$ o conjunto formado por exatamente um elemento de cada classe de equivalência em $\mathbf{Q}(\mathbf{B}(\psi))$, isto é, $\mathbf{E}(\psi)$ é um conjunto tal que $|\mathbf{E}(\psi)|=|\mathbf{Q}(\mathbf{B}(\psi))|$ e para cada $\mathbf{X} \in \mathbf{Q}(\mathbf{B}(\psi))$, existe $[A, B] \in \mathbf{E}(\psi)$ tal que $[A, B] \in \mathbf{X}$.

Seja $[A, B] \in \mathrm{E}(\psi)$, isto é, $[A, B]$ é um intervalo que representa um classe de equivalência. Denotaremos por $\mathbf{C}_{[A, B]}$ a classe de equivalência representada pelo intervalo $[A, B]$. Seguindo esta notação, temos que $\mathbf{Q}(\mathbf{B}(\psi))=\left\{\mathbf{C}_{[A, B]}:[A, B] \in \mathbf{E}(\psi)\right\}$.

Denotaremos por $C_{[A, B]}$ o subconjunto de $E$ que é composto por todos $h \in E$ tais que $[A, B]_{-h}$ está na classe de equivalência representada por $[A, B]$, isto é, para todo $[A, B] \in \mathbf{E}(\psi), C_{[A, B]}=\left\{h \in E:[A, B]_{-h} \in \mathbf{B}(\psi)\right\}$. Dessa forma, a classe de equivalência representada por $[A, B]$ é dada por $\mathbf{C}_{[A, B]}=\left\{[A, B]_{-h}: h \in C_{[A, B]}\right\}$. Note que, como $\mathbf{C}_{[A, B]} \subseteq \mathbf{B}(\psi)$ e $\mathbf{B}(\psi)$ é uma coleção de intervalos maximais, então, claramente, $\mathbf{C}_{[A, B]}$ 
é também uma coleção de intervalos maximais. Logo $\mathbf{C}_{[A, B]}=\left\{[A, B]_{-h}: h \in C_{[A, B]}\right\}=$ $\sqcup\left\{\left\{[A, B]_{-h}\right\}: h \in C_{[A, B]}\right\}=\sqcup\left\{\left\{[A, B]_{h}\right\}: h \in C_{[A, B]}^{t}\right\}=\sqcup\left\{\{[A, B]\}_{h}: h \in C_{[A, B]}^{t}\right\}$. Assim, pela definição de adição de Minkowski entre uma coleção de intervalos maximais e um conjunto, temos que $\mathbf{C}_{[A, B]}=\{[A, B]\} \oplus C_{[A, B]}^{t}$.

O teorema seguinte nos fornece a sup-representação compacta para qualquer $W$ operador $\psi$.

Teorema 8.1 Se $\psi \in \Psi_{W}$, então $\psi=\vee\left\{\delta_{C_{[A, B]}} \lambda_{A, B}^{W}:[A, B] \in \mathbf{E}(\psi)\right\}$.

Prova: Para todo $\psi \in \Psi_{W}$, temos que $\mathbf{Q}\left(\mathbf{B}_{W}(\psi)\right)=\left\{\mathbf{B}_{W}\left(\delta_{C_{[A, B]}} \lambda_{A, B}\right):[A, B] \in \mathbf{E}(\psi)\right\}$, uma vez que, para todo $[A, B] \in \mathbf{E}(\psi)$,

$$
\begin{aligned}
\mathbf{B}_{W}\left(\delta_{C_{[A, B]}} \lambda_{A, B}^{W}\right) & =\mathbf{B}_{W}\left(\lambda_{A, B}^{W}\right) \oplus C_{[A, B]}^{t} \\
& =\{[A, B]\} \oplus C_{[A, B]}^{t} \text { (pelas Proposições 3.24 e 3.41) } \\
& =\mathbf{C}_{[A, B]} .
\end{aligned}
$$

E a inf-representação compacta é dada pelo seguinte teorema.

Teorema 8.2 Se $\psi \in \Psi_{W}$, então $\psi=\wedge\left\{\varepsilon_{C_{[A, B]}^{t}} \mu_{A^{t}, B^{t}}^{W^{t}}:[A, B] \in \mathbf{E}\left(\psi^{*}\right)\right\}$.

Prova: Pelo Teorema 8.1, temos que, para todo $X \in \mathcal{P}(E)$,

$$
\psi^{*}(X)=\cup\left\{\delta_{C_{[A, B]}} \lambda_{A, B}(X):[A, B] \in \mathbf{E}\left(\psi^{*}\right\} .\right.
$$

Pela definição de $\psi^{*}$, temos que, $\psi=\nu \psi^{*} \nu$. Assim, para todo $X \in \mathcal{P}(E)$,

$$
\begin{aligned}
& \psi(X)=\nu \psi^{*}(\nu(X)) \\
& =\nu\left(\cup\left\{\delta_{C_{[A, B]}} \lambda_{A, B}^{W}(\nu(X)):[A, B] \in \mathbf{E}\left(\psi^{*}\right)\right\}\right) \\
& =\nu\left(\cup\left\{\delta_{\left.C_{[A, B]}\right]} \lambda_{A, B}^{W} \nu(X):[A, B] \in \mathbf{E}\left(\psi^{*}\right)\right\}\right) \\
& =\cap\left\{\nu \delta_{C_{[A, B]}} \lambda_{A, B}^{W} \nu(X):[A, B] \in \mathbf{E}\left(\psi^{*}\right)\right\} \\
& \text { (pela Lei de Morgan) } \\
& =\cap\left\{\nu \delta_{C_{[A, B]}} \nu \nu \lambda_{A, B}^{W} \nu(X):[A, B] \in \mathbf{E}\left(\psi^{*}\right)\right\} \\
& \text { (uma vez que } \nu \nu \text { é o operador identidade) } \\
& =\cap\left\{\left(\nu \delta_{C_{[A, B]}} \nu\right)\left(\nu \lambda_{A, B}^{W} \nu\right)(X):[A, B] \in \mathbf{E}\left(\psi^{*}\right)\right\} \\
& =\cap\left\{\varepsilon_{C_{[A, B]}^{t}} \mu_{A^{t}, B^{t}}^{W^{t}}(X):[A, B] \in \mathbf{E}\left(\psi^{*}\right)\right\} \\
& \text { (pelas Proposições } 3.30 \text { e } 3.34 \text { ) }
\end{aligned}
$$

Portanto, $\psi=\wedge\left\{\varepsilon_{C_{[A, B]}^{t}} \mu_{A^{t}, B^{t}}^{W^{t}}:[A, B] \in \mathbf{E}\left(\psi^{*}\right)\right\}$.

A sup-representação compacta pode ainda ser melhorada. De fato, se existe $\mathbf{X} \subseteq \mathbf{E}(\psi)$ tal que, para todo $[A, B] \in \mathrm{X}, C=C_{[A, B]}$, então, uma vez que $\delta_{C}$ comuta com o supremo (veja a Proposição 3.29 ), temos que 


$$
\vee\left\{\delta_{C_{[A, B]}} \lambda_{A, B}:[A, B] \in \mathbf{X}\right\}=\vee\left\{\delta_{C} \lambda_{A, B}:[A, B] \in \mathbf{X}\right\}=\delta_{C}\left(\vee\left\{\lambda_{A, B}:[A, B] \in \mathbf{X}\right\}\right) .
$$

Observe que, com esta fatorização, economizamos $|\mathbf{X}|-1$ dilatações.

\subsection{Representação Compacta para Operadores não Crescentes}

Nesta seção, mostraremos como a sup-representação (respectivamente, inf-representação) compacta é simplificada para $W$-operadores que são anti-extensivos (respectivamente, extensivos) e idempotentes em um sentido estrito.

Vimos no Capítulo 3 que um operador $\psi \in \Psi_{W}$ é chamado anti-extensivo se, e somente se, $\psi \leq \iota$, onde $\iota$ denota o operador identidade. Uma propriedade dos operadores antiextensivos $\psi \in \Psi_{W}$ é que, para todo $X \in \mathcal{K}(\psi)$, o $\in X$, uma vez que $o \in \psi(X) \subseteq X$. Portanto, $o \in A$, para todo $[A, B] \in \mathbf{B}(\psi)$.

Proposição 8.3 Se um operador $\psi \in \Psi_{W}$ é anti-extensivo, então, para todo $[A, B] \in$ $\mathbf{E}(\psi)$, temos que $C_{[A, B]} \subseteq A$.

Prova: Para todo $[A, B] \in \mathbf{E}(\psi), x \in C_{[A, B]}$ é equivalente a $[A, B]_{-x}=\left[A_{-x}, B_{-x}\right] \in$ $\mathrm{B}(\psi)$ e, uma vez que $\psi$ é anti-extensivo, $o \in A_{-x}$, temos que $x \in A$. Dessa forma, $C_{[A, B]} \subseteq A$.

Dizemos que um operador $\psi \in \Psi_{W}$ é autolimitado se para todo $[A, B] \in \mathbf{B}(\psi), X \in$ $[A, B]$ implica que $\psi(X) \cap W \in[A, B]$.

Lembramos o leitor que um operador $\psi \in \Psi_{W}$ é dito idempotente se, e somente se, $\psi \psi=\psi$. Por um lado, observe que a autolimitação não implica idempotência. Por exemplo, para qualquer $C \in \mathcal{P}(W)$ tal que $o \in C$, a dilatação $\delta_{C}$ é um operador autolimitado, mas $\delta_{C}$ não é idempotente. Por outro lado, um operador idempotente não é necessariamente autolimitado. Por exemplo, para todo $A, B \in \mathcal{P}(W)$ tais que $o \in A \subset B$ e $|B|-|A| \geq 2$, o operador $\psi=\delta_{A} \lambda_{A, A} \vee \delta_{A} \lambda_{B, B}$ é idempotente, mas não é autolimitado, uma vez que $B \in[B, B] \in \mathbf{B}(\psi)$ e $\psi(B)=A \notin[B, B]$.

Dizemos que um operador $\psi \in \Psi_{W}$ é um operador idempotente em um sentido estrito se, e somente se, $\psi$ é ao mesmo tempo idempotente e autolimitado.

A próxima proposição fornece uma condição suficiente para a idempotência de operadores não necessariamente crescentes.

Proposição 8.4 Se um operador $\psi \in \Psi_{W}$ é anti-extensivo e autolimitado, então $\psi$ é um operador idempotente. 
Prova: Por um lado, se $x \in \psi(\psi(X))$, então $o \in \psi\left(\psi\left(X_{-x}\right)\right)$, uma vez que $\psi$ é IT. Assim, temos que $o \in \psi\left(X_{-x}\right)$, uma vez que $\psi$ é anti-extensivo, e, conseqüentemente, $x \in \psi(X)$, uma vez que $\psi$ é IT. Por outro lado, se $x \in \psi(X)$, então $X_{-x} \cap W \in \mathcal{K}(\psi)$, uma vez que $\psi$ é IT e localmente definido em $W$. Assim, temos que existe um intervalo $[A, B] \in \mathbf{B}(\psi)$ tal que $X_{-x} \cap W \in[A, B]$. Logo, existe $[A, B] \in \mathbf{B}(\psi)$ tal que $\psi\left(X_{-x} \cap W\right) \cap W \in[A, B]$, uma vez que $\psi$ é autolimitado. Assim, $\psi\left(X_{-x} \cap W\right) \cap W \in \mathcal{K}(\psi)$ e, conseqüentemente, $o \in \psi\left(\psi\left(X_{-x} \cap W\right) \cap W\right)$. Dessa forma, como $\psi$ é localmente definido em $W$, temos que $o \in \psi\left(\psi\left(X_{-x} \cap W\right)\right)$ e $o \in \psi(\psi(X))$. Portanto, $x \in \psi(\psi(X))$, uma vez que $\psi$ é IT.

No teorema seguinte, provamos que operadores do tipo $\delta_{A} \lambda_{A, A}$, que foram extensivamente estudados por Ronse [39], constituem uma família de sup-geradores para operadores que são anti-extensivos e idempotentes em um sentido estrito. Para isto, apresentamos antes a seguinte proposição.

Proposição 8.5 Se um operador $\psi \in \Psi_{W}$ é autolimitado, então, para todo $[A, B] \in \mathbf{E}(\psi)$, temos que $\forall x \in A,\left[A_{-x}, B_{-x}\right] \subseteq \mathcal{K}(\psi)$.

Prova: Para todo $[A, B] \in \mathbf{E}(\psi)$ e para todo $Y \in[A, B]$, se $x \in A$, então $x \in \psi(Y) \cap W$, uma vez que $\psi$ é autolimitado e $A$ é o menor elemento de $[A, B]$. Assim, $x \in \psi(Y)$, e, dessa forma, $o \in \psi\left(Y_{-x}\right)$, uma vez que $\psi$ é IT, e, conseqüentemente, $\left[A_{-x}, B_{-x}\right] \subseteq \mathcal{K}(\psi)$.

Teorema 8.6 Se um operador $\psi \in \Psi_{W}$ é anti-extensivo e autolimitado, então

$$
\psi=\vee\left\{\delta_{A} \lambda_{A, B}:[A, B] \in \mathbf{E}(\psi)\right\} .
$$

Prova: Este teorema é uma conseqüência imediata das Proposições 8.3 e 8.5. A Proposição 8.3 garante que $\mathbf{B}(\psi)$ está completa e a Proposição 8.5 garante que todas outras possibilidades para esta representação estão incluídas em algum intervalo de $\mathbf{B}(\psi)$.

Recordamos o leitor que um operador $\psi \in \Psi_{W}$ é extensivo se, e somente se, $\iota \leq \psi$.

No próximo teorema, provamos que operadores do tipo $\varepsilon_{A} \mu_{A, B}$ constituem uma família de inf-geradores para todo operador $\psi \in \Psi_{W}$ tal que $\psi^{*}$ é anti-extensivo e idempotente em um sentido estrito.

Teorema 8.7 Se um operador $\psi \in \Psi_{W}$ tal que $\psi^{*}$ é anti-extensivo e autolimitado, então

$$
\psi=\wedge\left\{\varepsilon_{A^{t}} \mu_{A^{t}, B^{t}}^{W^{t}}:[A, B] \in \mathbf{E}\left(\psi^{*}\right)\right\} .
$$

Prova: Como $\psi^{*}$ é anti-extensivo e autolimitado, pelo teorema 8.6, temos que, para todo $X \in \mathcal{P}(E)$,

$$
\psi^{*}=\vee\left\{\delta_{A} \lambda_{A, B}:[A, B] \in \mathbf{E}\left(\psi^{*}\right)\right\}
$$


Como $\psi=\nu \psi^{*} \nu$, temos que, para todo $X \in \mathcal{P}(E)$,

$$
\begin{aligned}
& \psi(X)= \nu \psi^{*}(\nu(X)) \\
&= \nu\left(\cup\left\{\delta_{A} \lambda_{A, B}^{W}(\nu(X)):[A, B] \in \mathbf{E}\left(\psi^{*}\right)\right\}\right) \\
&= \nu\left(\cup\left\{\delta_{A} \lambda_{A, B}^{W} \nu(X):[A, B] \in \mathbf{E}\left(\psi^{*}\right)\right\}\right) \\
&= \cap\left\{\nu \delta_{A} \lambda_{A, B}^{W} \nu(X):[A, B] \in \mathbf{E}\left(\psi^{*}\right)\right\} \\
&= \quad \cap\left\{\nu \delta_{A} \nu \nu \lambda_{A, B}^{W} \nu(X):[A, B] \in \mathbf{E}\left(\psi^{*}\right)\right\} \\
& \quad(\text { uma vez que } \nu \nu \text { é o operador identidade) } \\
&= \cap\left\{\left(\nu \delta_{A} \nu\right)\left(\nu \lambda_{A, B}^{W} \nu\right)(X):[A, B] \in \mathbf{E}\left(\psi^{*}\right)\right\} \\
&= \cap\left\{\varepsilon_{A^{t}} \mu_{A^{t}, B^{t}}^{W^{t}}(X):[A, B] \in \mathbf{E}\left(\psi^{*}\right)\right\} \\
& \text { (pelas Proposições 3.30 e 3.34) }
\end{aligned}
$$

Portanto, $\psi=\wedge\left\{\varepsilon_{A^{t}} \mu_{A^{t}, B^{t}}^{W^{t}}:[A, B] \in \mathbf{E}\left(\psi^{*}\right)\right\}$.

Observamos que operadores anti-extensivos (respectivamente, extensivos) e idempotentes que não são autolimitados podem não ser representados pelo Teorema 8.6 (respectivamente, Teorema 8.7). O último exemplo antes da Proposição 8.4 prova isto.

Note que a implementação computacional de operadores anti-extensivos que são autolimitados pela decomposição do Teorema 8.1 é mais eficiente que a implementação pela decomposição do Teorema 8.6, uma vez que, para todo $[A, B] \in \mathbf{E}(\psi), C_{[A, B]} \subseteq A$.

\subsection{Representação Compacta para Aberturas e Fe- chamentos Invariantes por Translação}

Nesta seção mostraremos que a representação compacta de aberturas e fechamentos IT's é uma realização eficiente da representação de Matheron dada no Capítulo 3, Subseção 3.6.2.

Vimos na Subseção 3.6 .1 que se $\psi \in \Psi_{W}$ é um operador crescente, então os operadores $\lambda_{A, B}$ e $\mu_{A, B}$ das famílias de sup-geradores e inf-geradores dos Teoremas 3.38 e 3.39 tornamse, respectivamente, erosões e dilatações. Conseqüentemente, os operadores $\lambda_{A, B}$ e $\mu_{A, B}$ da representação compacta dados nos Teoremas 8.1 e 8.2 tornam-se erosões e dilatações.

Seja $\psi \in \Psi_{W}$ uma abertura. Como $\psi$ é crescente, anti-extensivo e idempotente, para todo $[A, B] \in \mathbf{B}(\psi), A$ é um invariante de $\psi$ (isto é, $\psi(A)=A$ ), e, conseqüentemente, $\psi$ é autolimitado. Como $\psi$ é crescente, anti-extensivo e autolimitado, a estrutura de decomposição do Teorema 8.6 reduz-se a um supremo de aberturas morfológicas. Tomando $W=E$, esta é a realização do resultado clássico de Matheron para representação de aberturas IT's (Teorema 3.48.a). Além disso, a proposição seguinte mostra que esta é uma realização minimal, no sentido da absorção granulométrica, isto é, aberturas morfológicas que são absorvidas por outras aberturas morfológicas não estão consideradas nesta decomposição.

Proposição 8.8 Seja $\psi \in \Psi_{W}$ uma abertura. Se $[A, W] \in \mathbf{B}(\psi)$, então não existem 
$h \in E$ e $A^{\prime} \in \mathcal{P}(W)$ tais que $A^{\prime} \neq A, A^{\prime}$ é um invariante de $A$ e $\left[A_{h}^{\prime}, W\right] \in \mathbf{B}(\psi)$.

Prova: Seja $[A, W] \in \mathbf{B}(\psi)$. Suponha por absurdo que exista $h \in E$ e $A^{\prime} \in \mathcal{P}(W)$ tais que $A^{\prime} \neq A, A^{\prime}$ é invariante de $A$ e $\left[A_{h}^{\prime}, W\right] \in \mathbf{B}(\psi)$.

Como $\left[A_{h}^{\prime}, W\right]$ e $[A, W]$ pertencem a $\mathbf{B}(\psi)$ e $A^{\prime} \neq A$, então podemos escolher $\mathbf{E}(\psi)$ tal que $\left[A_{h}^{\prime}, W\right],[A, W] \in \mathbf{E}(\psi)$. Pela independência da translação de $\gamma_{A^{\prime}}$ e pela simplificação do Teorema 8.6 para aberturas IT's, temos que $\gamma_{A_{h}^{\prime}}=\gamma_{A^{\prime}} \leq \psi$. Como $A^{\prime}$ é um invariante de $A$ e $A^{\prime} \neq A$, então, pela Proposição $3.49, \gamma_{A}<\gamma_{A^{\prime}}$. Pela Proposição 3.27 , temos que $\mathcal{K}(\cdot)$ é um isomorfismo de reticulado entre $\left(\Psi_{W}, \leq\right)$ e $(\mathcal{P}(\mathcal{P}(W)), \subseteq)$. Assim, $\gamma_{A}<\gamma_{A^{\prime}}=$ $\gamma_{A_{h}^{\prime}} \leq \psi \Leftrightarrow \mathcal{K}\left(\gamma_{A}\right) \subset \mathcal{K}\left(\gamma_{A^{\prime}}\right)=\mathcal{K}\left(\gamma_{A_{h}^{\prime}}\right) \subseteq \mathcal{K}(\psi)$.

Uma vez que $[A, W] \in \mathbf{E}(\psi)$ e $\psi$ é uma abertura (conseqüentemente, $\psi$ é antiextensivo), então $o \in A$.

Pela Proposição 3.47, temos que $\mathcal{K}\left(\gamma_{A}\right)=\left\{X \in \mathcal{P}(W): A_{-z} \subseteq X\right.$ e $\left.z \in A\right\}$ e $\mathcal{K}\left(\gamma_{A^{\prime}}\right)=\left\{X \in \mathcal{P}(W): A_{-z}^{\prime} \subseteq X\right.$ e $\left.z \in A^{\prime}\right\}$. Assim, como $o \in A$, então $A \in \mathcal{K}\left(\gamma_{A}\right)$. Além disso, como $A \in \mathcal{K}\left(\gamma_{A}\right) \subset \mathcal{K}\left(\gamma_{A^{\prime}}\right) \subseteq \mathcal{K}(\psi)$, então existe $z \in A^{\prime}$ tal que $A_{z}^{\prime} \subset A$ e $A_{z}^{\prime} \in \mathcal{K}(\psi)$. Dessa forma, $\left[A_{z}^{\prime}, W\right] \in \mathbf{B}(\psi)$ e, portanto, temos que $[A, W] \notin \mathbf{B}(\psi)$, uma vez que $[A, W]$ não é um intervalo maximal em $\mathbf{B}(\psi)$. Mas isto contradiz a hipótese de que $[A, W] \in \mathbf{B}(\psi)$. Portanto, não existem tais $A^{\prime}$ e $h$.

Seja $\psi \in \Psi_{W}$ um fechamento. Como $\psi$ é crescente, extensivo e idempotente, então, pela Proposição 3.18, $\psi^{*}$ é crescente, anti-extensivo e idempotente (isto é, $\psi^{*}$ é uma abertura). Assim, para todo $[A, B] \in \mathbf{B}\left(\psi^{*}\right), A$ é um invariante de $\psi^{*}$ (isto é, $\psi^{*}(A)=A$ ) e, conseqüentemente, $\psi^{*}$ é autolimitado. Como $\psi^{*}$ é crescente, anti-extensivo e autolimitado, então a estrutura de decomposição do Teorema 8.7 reduz $\psi$ a um ínfimo de fechamentos morfológicos, uma vez que se $B=W$ os operadores $\mu_{A, B}$ da representação compacta tornam-se dilatações. Tomando $W=E$, esta é a realização do resultado clássico de Matheron para representação de fechamentos IT's (Teorema 3.48.b). Além disso, a proposição seguinte mostra que esta é uma realização minimal, no sentido da absorção granulométrica, isto é, fechamentos morfológicas que são absorvidas por outros fechamentos morfológicos não estão considerados nesta decomposição.

Proposição 8.9 Seja $\psi \in \Psi_{W}$ um fechamento. Se $[A, W] \in \mathrm{B}\left(\psi^{*}\right)$, então não existem $h \in E$ e $A^{\prime} \in \mathcal{P}(W)$ tais que $A^{\prime} \neq A, A^{\prime}$ é um invariante de $A$ e $\left[A_{h}^{\prime}, W\right] \in \mathbf{B}\left(\psi^{*}\right)$.

Prova: Como $\psi^{*}$ é uma abertura, pela Proposição 8.8, não existem $h \in E$ e $A^{\prime} \in \mathcal{P}(W)$ tais que $A^{\prime} \neq A, A^{\prime}$ é um invariante de $A$ e $\left[A_{h}^{\prime}, W\right] \in \mathbf{B}\left(\psi^{*}\right)$. 


\subsection{Conversão da Sup-Representação para a Repre- sentação Compacta}

Foi implementado um programa que transforma automaticamente a sup-representação de um operador para a sua sup-representação compacta. A tarefa deste programa é primeiro identificar as classes de equivalência na base do operador e depois identificar as que podem ser fatoradas por ter o mesmo $C_{[A, B]}$.

Observe que no caso de operadores anti-extensivos que são autolimitados, temos a flexibilidade de escolher $C_{[A, B]}$ como qualquer $C$ tal que $C_{[A, B]} \subseteq C \subseteq A$. A idéia é escolher um $C$ que maximiza o efeito de fatoração.

A seguir, damos alguns exemplos da aplicação deste programa para alguns exemplos simples. Para estes exemplos, considere $A=1 \underline{11}, B=\frac{1}{1}, C=\frac{110}{01} \frac{1}{1}, D=\frac{1}{1}, F=1 \underline{0} 1$ e $G=0 \underline{11}$.

Seja $\psi_{1}=\delta_{A} \varepsilon_{A} \vee \delta_{B} \varepsilon_{B}$. A base de $\psi_{1}$ é $\mathbf{B}\left(\psi_{1}\right)=\left\{\left[A_{a}, W\right]: a \in A\right\}+\left\{\left[B_{b}, W\right]: b \in B\right\}$, onde + denota a união de conjuntos disjuntos. Logo, neste caso, o operador dado já está na forma da representação compacta. Agora, seja $\psi_{2}=\delta_{A} \varepsilon_{A} \vee \delta_{C} \varepsilon_{C}$. A base de $\psi_{2}$ é $\mathbf{B}\left(\psi_{2}\right)=\left\{\left[A_{a}, W\right]: a \in A\right\}+\left\{\left[C_{d}, W\right]: d \in D\right\}$. Logo, $\psi_{2}$ pode ser reescrito como $\psi_{2}=\delta_{A} \varepsilon_{A} \vee \delta_{D} \varepsilon_{C}$. Note que $\psi_{1}$ e $\psi_{2}$ são ambos operadores crescentes e a representação compacta de $\psi_{2}$ é melhor que a original, uma vez que $|D|<|C|$.

Seja $\psi_{3}=\delta_{A} \lambda_{A, A} \vee \delta_{C} \lambda_{C, C}$. A base de $\psi_{3}$ é $\mathbf{B}\left(\psi_{3}\right)=\left\{\left[A_{a}, A_{a}\right]: a \in A\right\}+\left\{\left[C_{c}, C_{c}\right]:\right.$ $c \in C\}$. Logo, o operador já está na forma da representação compacta. Agora, seja $\psi_{4}=\delta_{A} \lambda_{A, C} \vee \delta_{C} \lambda_{C, C}$. A base de $\psi_{4}$ é $\mathbf{B}\left(\psi_{4}\right)=\left\{\left[A_{a}, C_{a}\right]: a \in A\right\}+\left\{\left[C_{d}, C_{d}\right]: d \in D\right\}$. Logo, $\psi_{4}$ pode ser reescrito como $\psi_{4}=\delta_{A} \lambda_{A, C} \vee \delta_{D} \lambda_{C, C}$. Observe que $\psi_{3}$ e $\psi_{4}$ são ambos operadores não crescentes e a representação compacta de $\psi_{4}$ é melhor que a original, uma vez que $|D|<|C|$.

Seja $\psi_{5}=\delta_{F} \lambda_{F, C} \vee \delta_{G} \lambda_{G, C}$. A base de $\psi_{5}$ é $\mathbf{B}\left(\psi_{5}\right)=\left\{\left[F_{f}, C_{f}\right]: f \in F^{t}\right\}+\left\{\left[G_{g}, C_{g}\right]\right.$ : $\left.g \in G^{t}\right\}$. Mas, $G^{t}=\left(F^{t}\right)_{h}$, onde $h=(-1,0)$. Portanto, $\mathbf{B}\left(\psi_{5}\right)=\left\{\left[F_{f}, C_{f}\right]: f \in\right.$ $\left.F^{t}\right\}+\left\{\left[F_{f}, C_{h+f}\right]: f \in F^{t}\right\}$ Logo, $\psi_{5}$ pode ser reescrito como $\psi_{5}=\delta_{F} \lambda_{F, C} \vee \delta_{F} \lambda_{F, C_{h}}=$ $\delta_{F}\left(\lambda_{F, C} \vee \lambda_{F, C_{h}}\right)$. Neste caso, com esta fatoração, economizamos uma dilatação.

\subsection{Conclusão}

Introduzimos uma nova sup-representação e inf-representação para $W$-operadores: a representação compacta. A família de sup-geradores (respectivamente, inf-geradores) nesta representação é constituída de composições de sup-geradores da sup-decomposição com dilatações (respectivamente, de composições de inf-geradores da inf-decomposição com erosões). 
Uma propriedade importante da representação compacta é que ela pode usar um número menor (ou, no pior caso, o mesmo número) de operadores sup-geradores (respectivamente, inf-geradores) que na sup-decomposição (respectivamente, inf-decomposição). Esta propriedade implica que os operadores são mais eficientes quando implementados na representação compacta.

Apresentamos também uma simplificação da representação compacta para a família de operadores idempotentes em um sentido estrito e anti-extensivos (extensivos). Além disso, a partir desta forma simplificada, obtemos uma realização minimal da representação de Matheron para aberturas e fechamentos IT's.

Finalmente, mostramos que a sup-decomposição pode ser facilmente transformada para a sup-representação compacta e apresentamos alguns exemplos dessa transformação. 


\section{Capítulo 9}

\section{Sup-Decomposição para Decomposições Seqüenciais}

Como já vimos no Capítulo 3, a sup-decomposição tem algumas boas propriedades como caracterizar unicamente os $W$-operadores e possuir uma estrutura simples que pode ser facilmente utilizada para projeto automático de operadores. No entanto, esta representação tem um sério inconveniente: sua estrutura paralela não é eficiente para implementação em máquinas convencionais seqüenciais.

Neste capítulo, formalizamos o problema de transformar as sup-decomposições em decomposições puramente seqüenciais (quando elas existem). Uma versão especializada deste problema é o problema clássico de encontrar decomposições seqüenciais para erosões e dilatações (veja os Capítulos 4, 5, 6 e 7).

A teoria proposta consiste na formulação e solução de equações discretas em reticulados completos, cujo espaço de soluções tem uma forte natureza combinatória. As técnicas foram desenvolvidas para $W$-operadores em geral, depois, especializadas para $W$-operadores crescentes e, posteriormente, aplicadas sobre operadores construídos por composições de dilatações e erosões.

Os resultados apresentados aqui para $W$-operadores em geral são uma extensão de alguns resultados obtidos por Jones [27] para operadores crescentes IT's e introduzem alguns novos limites superiores e inferiores para o caso particular de $W$-operadores crescentes.

\subsection{Propriedades de Coleções de Intervalos Maximais}

Nesta seção, veremos algumas propriedades sobre coleções de intervalos maximais que serão usadas neste capítulo.

Proposição 9.1 Se $\mathrm{X} \in \Pi_{W}, C \in \mathcal{P}(E)$ e $h \in E$, então $\mathrm{X}_{h} \oplus C=\mathrm{X} \oplus C_{h}=(\mathrm{X} \oplus C)_{h}$. 
Prova: Primeiramente, mostramos que $(\mathrm{X} \oplus C)_{h}=\mathrm{X}_{h} \oplus C$.

$$
\begin{aligned}
(\mathrm{X} \oplus C)_{h} & =\left(\sqcup\left\{\mathbf{X}_{c}: c \in C\right\}\right)_{h} \\
& =\sqcup\left\{\mathbf{X}_{c+h}: c \in C\right\} \\
& =\sqcup\left\{\left(\mathbf{X}_{h}\right)_{c}: c \in C\right\} \\
& =\mathrm{X}_{h} \oplus C .
\end{aligned}
$$

Agora, provamos que $(\mathrm{X} \oplus C)_{h}=\mathrm{X} \oplus C_{h}$.

$$
\begin{aligned}
(\mathrm{X} \oplus C)_{h} & =\left(\sqcup\left\{\mathbf{X}_{c}: c \in C\right\}\right)_{h} \\
& =\sqcup\left\{\mathbf{X}_{c+h}: c \in C\right\} \\
& =\sqcup\left\{\mathbf{X}_{d}: d \in C_{h}\right\} \\
& =\mathrm{X} \oplus C_{h} .
\end{aligned}
$$

Proposição 9.2 Se X, Y $\in \Pi_{W}$ e $C \in \mathcal{P}(E)$, então

$$
(\mathbf{X} \sqcup \mathbf{Y}) \oplus C=(\mathbf{X} \oplus C) \sqcup(\mathbf{Y} \oplus C) e(\mathbf{X} \sqcap \mathbf{Y}) \ominus C=(\mathbf{X} \ominus C) \sqcap(\mathbf{Y} \ominus C) \text {. }
$$

Prova:

$$
\begin{aligned}
(\mathrm{X} \sqcup \mathrm{Y}) \oplus C & =\sqcup\left\{\left(\mathbf{X} \sqcup \mathrm{Y}_{h}: h \in C\right\}\right. \\
& =\sqcup\left\{\left(\mathrm{X}_{h} \sqcup \mathbf{Y}_{h}\right): h \in C\right\} \\
& =\left(\sqcup\left\{\mathbf{X}_{h}: h \in C\right\}\right) \sqcup\left(\sqcup\left\{\mathbf{Y}_{h}: h \in C\right\}\right) \\
& \quad \text { (pela Proposição 3.23.c) } \\
& =(\mathrm{X} \oplus C) \sqcup(\mathbf{Y} \oplus C) .
\end{aligned}
$$

Analogamente, pode-se provar que $(\mathrm{X} \sqcap \mathrm{Y}) \ominus C=(\mathrm{X} \ominus C) \sqcap(\mathrm{Y} \ominus C)$.

Como conseqüência da Proposição 9.2, temos o seguinte resultado.

Proposição 9.3 Sejam X, Y $\in \Pi_{W} e C \in \mathcal{P}(E)$. Se $\mathrm{X} \leq \mathrm{Y}$, então

$$
\mathrm{X} \oplus C \leq \mathrm{Y} \oplus C \text { e } \mathrm{X} \ominus C \leq \mathrm{Y} \ominus C \text {. }
$$

\section{Prova:}

$$
\begin{aligned}
\mathrm{X} \leq \mathrm{Y} & \Leftrightarrow \mathrm{X} \sqcup \mathrm{Y}=\mathrm{Y} \\
& \Leftrightarrow(\mathrm{X} \sqcup \mathrm{Y}) \oplus C=\mathrm{Y} \oplus C \\
& \Rightarrow(\mathrm{X} \oplus C) \sqcup(\mathrm{Y} \oplus C)=\mathrm{Y} \oplus C \\
& \Leftrightarrow(\mathrm{X} \oplus C) \leq \mathrm{Y} \oplus C .
\end{aligned}
$$

Analogamente, pode-se provar que $\mathrm{X} \ominus C \leq \mathrm{Y} \ominus C$.

Proposição 9.4 Se $\mathbf{X}_{W} \in \Pi_{W}$ e $C \in \mathcal{P}(E)$, então, para todo $h \in E, \mathbf{X}_{W} \oplus C=$ $\left(\mathrm{X}_{-h}\right)_{W_{-h}} \oplus C_{h}$. 
Prova: Sejam $W^{\prime} \in \mathcal{P}(E)$ e $\mathrm{Y}_{W^{\prime}} \in \Pi_{W^{\prime}}$ tais que $\mathrm{Y}_{W^{\prime}}=\mathrm{X}_{W} \oplus C$. Assim,

$$
\begin{aligned}
\mathrm{X} \oplus C=\mathrm{Y} & \Leftrightarrow(\mathrm{X} \oplus C)_{h}=\mathrm{Y}_{h} \\
& \Leftrightarrow \mathrm{X} \oplus C_{h}=\mathrm{Y}_{h} \\
& \Leftrightarrow\left(\mathrm{X} \oplus C_{h}\right)_{-h}=\mathrm{Y} \\
& \Leftrightarrow \mathrm{X}_{-h} \oplus \mathrm{C}_{h}=\mathrm{Y}
\end{aligned}
$$

(pela Proposição 9.1)

(pela Proposição 9.1).

\subsection{Equação de Fatoração de Minkowski}

Nesta seção definimos o seguinte problema:

Problema: Dada uma coleção de intervalos maximais $\mathbf{Y}_{W^{\prime}} \in \Pi_{W^{\prime}}$ e um conjunto $C \in$ $\mathcal{P}(E)$, encontrar todas coleçôes de intervalos maximais $\mathbf{X}_{W} \in \Pi_{W}$ tais que

$$
\mathrm{X}_{W} \oplus C^{t}=\mathrm{Y}_{W^{\prime}}
$$

Uma vez que $\mathrm{X}_{W} \oplus C^{t} \in \Pi_{W \oplus C^{t}}$ e $\mathrm{Y}_{W^{\prime}} \in \Pi_{W^{\prime}}$, então $W$ e $W^{\prime}$ satisfazem $W \oplus C^{t}=W^{\prime}$.

\subsubsection{Limite Superior para $W$}

No próximo resultado temos um limite superior para $W$ na Equação (1) e é uma conseqüência direta da relação de adjunção dada pela Proposição 3.11.

Proposição 9.5 Sejam $W, C, W^{\prime} \in \mathcal{P}(E)$. Se $W \oplus C^{t}=W^{\prime}$, então $W \subseteq W^{\prime} \ominus C^{t}$.

Dado um conjunto $C \in \mathcal{P}(E)$ e uma coleção de intervalos maximais $\mathrm{Y}_{W^{\prime}}$, pelas Proposições 9.5 e 3.24, as coleções $\mathbf{X}_{W} \in \Pi_{W}$ que satisfazem a Equação (1) podem ter suas representações mudadas para $\mathbf{X}_{W^{\prime} \ominus C^{t}}$. Agora, é suficiente considerar $W=W^{\prime} \ominus C^{t}$. De fato, se existe uma solução $\mathbf{X}_{W} \in \Pi_{W}$ tal que $W \subset W^{\prime} \ominus C^{t}$, então existe uma solução $\mathbf{X}_{W^{\prime} \ominus C^{t}} \in \Pi_{W^{\prime} \ominus C^{t} / W}$ tal que $\mathcal{W}^{-1}\left(\mathrm{X}_{W^{\prime} \ominus C^{t}}\right)=\mathrm{X}_{W}$. Assim, podemos considerar que $W=W^{\prime} \ominus C^{t}$.

\subsubsection{Limite Superior para $\mathrm{X}_{W}$}

Nesta subseção, vamos mostrar um limite superior para $\mathrm{X}_{W}$. Seja $C \in \mathcal{P}(E)$. Uma conseqüência imediata da definição da adição de Minkowski é que $o \in C^{t} \oplus C$. Assim, para todo $W \in \mathcal{P}(E)$, podemos ver facilmente que $W \subseteq W \oplus\left(C^{t} \oplus C\right)$. Dessa forma, pela propriedade associativa da adição de Minkowski (Proposição 3.8.f), $W \subseteq\left(W \oplus C^{t}\right) \oplus$ $C$. Logo, pela Proposição 3.24 , podemos mudar a representação de qualquer coleção de intervalos maximais $\mathbf{X}_{W} \in \Pi_{W}$ para $\mathbf{X}_{W^{\prime \prime}} \in \Pi_{W^{\prime \prime}}$, onde $W^{\prime \prime}=\left(W \oplus C^{t}\right) \oplus C$. 
$W^{\prime}=11 \underline{11} \quad C=\underline{011}$

(a) (b)

$$
\begin{aligned}
& {\left[A_{1}^{\prime}, B_{1}^{\prime}\right]=[00 \underline{1}, 01 \underline{11}] \quad\left[A_{2}^{\prime}, B_{2}^{\prime}\right]=[00 \underline{10}, 01 \underline{1} 1] \quad\left[A_{3}^{\prime}, B_{3}^{\prime}\right]=[01 \underline{00}, 01 \underline{11}]} \\
& {\left[A_{4}^{\prime}, B_{4}^{\prime}\right]=[00 \underline{1} 1,10 \underline{1} 1] \quad\left[A_{5}^{\prime}, B_{5}^{\prime}\right]=[00 \underline{0} 0,10 \underline{1} 1] \quad\left[A_{6}^{\prime}, B_{6}^{\prime}\right]=[00 \underline{01} 11 \underline{10} 1]} \\
& {\left[A_{7}^{\prime}, B_{7}^{\prime}\right]=[01 \underline{00}, 11 \underline{1} 1] \quad\left[A_{8}^{\prime}, B_{8}^{\prime}\right]=[00 \underline{1} 0,11 \underline{1} 0] \quad\left[A_{9}^{\prime}, B_{9}^{\prime}\right]=[01 \underline{00}, 11 \underline{1} 0]} \\
& \text { (c) } \\
& \mathbf{Y}_{W^{\prime}}=\left\{\left[A_{1}^{\prime}, B_{1}^{\prime}\right],\left[A_{2}^{\prime}, B_{2}^{\prime}\right],\left[A_{3}^{\prime}, B_{3}^{\prime}\right],\left[A_{4}^{\prime}, B_{4}^{\prime}\right],\left[A_{5}^{\prime}, B_{5}^{\prime}\right],\left[A_{6}^{\prime}, B_{6}^{\prime}\right],\left[A_{7}^{\prime}, B_{7}^{\prime}\right],\left[A_{8}^{\prime}, B_{8}^{\prime}\right],\left[A_{9}^{\prime}, B_{9}^{\prime}\right]\right\} \\
& \text { (d) } \\
& W=W^{\prime} \ominus C^{t}=1 \underline{11} \quad W^{\prime \prime}=\left(W \oplus C^{t}\right) \oplus C=11 \underline{11} \\
& \text { (e) } \\
& (f) \\
& Y_{W^{\prime}} \Theta C^{t}=\{[01 \underline{001}, 11 \underline{1101]},[00100,11 \underline{101}],[01001,11 \underline{011}],[00 \underline{0} 10,11 \underline{11}],
\end{aligned}
$$

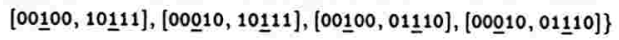

$$
\begin{aligned}
& \text { (g) } \\
& U_{W^{\prime \prime}}=\{(00 \underline{100}, 11 \underline{101}],[00 \underline{0} 10,11 \underline{011}],[00 \underline{100}, 10 \underline{111}],[00 \underline{010}, 10 \underline{11} 1]\} \\
& \text { (h) } \\
& \mathrm{U}_{W}=\{[0 \underline{10}, 1 \underline{11}],[0 \underline{01} 1,1 \underline{10}],[0 \underline{10} 0,011],[0 \underline{01}, 0 \underline{11}]\} \\
& \text { (i) } \\
& X_{1}=\{[0 \underline{10}, 110],[0 \underline{0} 1,101],[010,011],[0 \underline{10} 1,0 \underline{1} 1]\} \\
& \mathrm{X}_{2}=\{[0 \underline{10}, 1 \underline{1} 0],[0 \underline{10} 0,0 \underline{11}],[0 \underline{01}, 0 \underline{11}]\} \\
& \text { (j) }
\end{aligned}
$$

Figura 9.1: Limite superior para $\mathrm{X}_{W}$.

O próximo teorema mostra um limite superior para todos $\mathbf{X}_{W^{\prime \prime}} \in \Pi_{W^{\prime \prime}}$.

Teorema 9.6 Sejam $\mathbf{Y}_{W^{\prime}} \in \Pi_{W^{\prime}}$ e $C \in \mathcal{P}(E)$. Para todo $\mathbf{X}_{W} \in \Pi_{W}$ tal que $W \oplus C^{t}=W^{\prime}$ e $\mathbf{X}_{W} \oplus C^{t}=\mathbf{Y}_{W^{\prime}}$, temos que $\mathbf{X}_{W^{\prime \prime}} \leq \mathbf{Y}_{W^{\prime}} \ominus C^{t}$, onde $W^{\prime \prime}=\left(W \oplus C^{t}\right) \oplus C$.

\section{Prova:}

$$
\begin{aligned}
& \mathrm{X}_{W} \oplus C^{t}=\mathrm{Y}_{W^{\prime}} \Leftrightarrow \sqcup\left\{\left(\mathrm{X}_{h}\right)_{W \oplus C^{t}}: h \in C^{t}\right\}=\mathrm{Y}_{W^{\prime}} \\
& \Rightarrow \quad\left(\mathrm{X}_{h}\right)_{W^{\prime}} \leq \mathrm{Y}_{W^{\prime}} \\
& \Leftrightarrow \quad \mathrm{X}_{W_{-h}^{\prime}} \leq\left(\mathrm{Y}_{-h}\right)_{W_{-h}^{\prime}} \\
& \Leftrightarrow \quad \mathrm{X}_{W^{\prime} \oplus C} \leq\left(\mathrm{Y}_{-h}\right)_{W^{\prime} \oplus C} \\
& \text { (uma vez que } h \in C^{t} \text { e } W_{-h}^{\prime} \subseteq W^{\prime} \oplus C \text { ) } \\
& \Leftrightarrow \quad \mathrm{X}_{W^{\prime} \oplus C} \leq \sqcap\left\{\left(\mathrm{Y}_{-h}\right)_{W^{\prime} \oplus C}: h \in C^{t}\right\} \\
& \Leftrightarrow \mathrm{X}_{W^{\prime \prime}} \leq \mathrm{Y}_{W^{\prime}} \ominus C^{t} \text {. }
\end{aligned}
$$

Seja $\mathbf{X}_{W}$ uma solução da Equação (1). Assim, $\mathbf{X}_{W^{\prime \prime}} \in \Pi_{W^{\prime \prime} / W}$. Pelo Teorema 9.6, $\mathbf{X}_{W^{\prime \prime}} \leq \mathbf{Y}_{W^{\prime}} \ominus C^{t}$. Dessa forma, obtemos um limite superior para $\mathbf{X}_{W^{\prime \prime}}$. Agora, mostraremos um limite superior para $\mathbf{X}_{W}$. Uma vez que $\mathbf{X}_{W^{\prime \prime}} \leq \mathbf{Y}_{W^{\prime}} \ominus C^{t}$, então, para todo $[A, B] \in \mathbf{X}_{W^{\prime \prime}}$ existe $[L, R] \in \mathbf{Y}_{W^{\prime}} \ominus C^{t}$ tal que $[A, B] \subseteq[L, R]$. Como $\mathbf{Y}_{W^{\prime}} \ominus C^{t} \in \Pi_{W^{\prime \prime}}$, $L \subseteq A$ e $B \subseteq R \subseteq W^{\prime \prime}$. Uma vez que $[A, B] \in \mathrm{X}_{W^{\prime \prime}}$ e $\mathrm{X}_{W^{\prime \prime}} \in \Pi_{W^{\prime \prime} / W}$, então $A \subseteq W$ e $B-W=W^{c}$. Assim, $L \subseteq A \subseteq W$ e $W^{c}=B-W \subseteq R-W \subseteq W^{\prime \prime}-W=W^{c}$. 
Dessa forma, $R-W=W^{c}$, e, conseqüentemente, $\{[L, R]\} \in \Pi_{W^{\prime \prime} / W}$. Logo, para todo intervalo $[A, B] \in \mathbf{X}_{W^{\prime \prime}}$, existe um intervalo $[L, R] \in \mathrm{Y}_{W^{\prime}} \ominus C^{t}$, tal que $[A, B] \subseteq[L, R]$ and $\{[L, R]\} \in \Pi_{W^{\prime \prime} / W}$. Devemos alertar que pode existir um intervalo $[L, R] \in \mathbf{Y}_{W^{\prime}} \ominus C^{t}$ tal que $[L, R] \notin \Pi_{W^{\prime \prime} / W}$ (por exemplo, veja a Figura 9.1). Portanto, se $\mathbf{U}_{W^{\prime \prime}}=\{[L, R] \in$ $\left.\mathrm{Y}_{W^{\prime}} \ominus C^{t}:\{[L, R]\} \in \Pi_{W^{\prime \prime} / W}\right\}$ e $\mathbf{U}_{W}=\mathcal{W}^{-1}\left(\mathbf{U}_{W^{\prime \prime}}\right)$, então, para todo $\mathbf{X}_{W}$ que satisfaz a Equação (1), temos que $\mathbf{X}_{W} \leq \mathbf{U}_{W}$. Conseqüentemente, $\mathbf{U}_{W}$ é um limite superior para $\mathrm{X}_{W}$. Por exemplo, sejam $W^{\prime}$ e $C$ os dois conjuntos apresentados nas Figuras 9.1.a e 9.1.b. Seja $\mathbf{Y}_{W^{\prime}}$ a coleção de intervalos maximais apresentada na Figura 9.1.d. A coleção de intervalos maximais $\mathrm{Y}_{W^{\prime}} \ominus C^{t}$ está mostrada na Figura 9.1.g e os limites superiores $\mathrm{U}_{W^{\prime \prime}}$ para $\mathbf{X}_{W^{\prime \prime}}$ e $\mathbf{U}_{W}$ para $\mathbf{X}_{W}$ são os que estão apresentados na Figura 9.1.h e 9.1.i, respectivamente. Note que somente quatro intervalos de $\mathbf{Y}_{W^{\prime}} \ominus C^{t}$. aparecem em $\mathbf{U}_{W^{\prime \prime}}$. A Figura 9.1.j apresenta duas soluções para a Equação (1).

\subsubsection{Limites Inferiores para $\mathrm{X}_{W}$}

Nesta subseção, construiremos um conjunto $\Theta_{W^{\prime}}^{\mathbf{Y}_{W^{\prime}}, C} \subseteq \Pi_{W}$, onde, para cada solução $\mathbf{X}_{W}$ da Equação (1), existe um elemento $\mathbf{Z}_{W} \in \Theta_{W}^{\mathbf{Y}_{W^{\prime}}, C}$ tal que $\mathbf{Z}_{W} \leq \mathbf{X}_{W}$. Para isso, precisamos do seguinte resultado.

Proposição 9.7 Sejam $\mathrm{Y}_{W^{\prime}} \in \Pi_{W^{\prime}}, \mathrm{X}_{W} \in \Pi_{W}$ e $C \in \mathcal{P}(E)$. Se $\mathrm{X}_{W} \oplus C^{t}=\mathrm{Y}_{W^{\prime}}$, então, para cada intervalo $\left[A^{\prime}, B^{\prime}\right] \in \mathbf{Y}_{W^{\prime}}$, existem $[P, Q],[X, Y] \in \mathbf{X}_{W}$ e a,b $\in C^{t}$ tais que $P_{a}=A^{\prime}$ e $Y_{b}=B^{\prime}-\left(W_{b}\right)_{W^{\prime}}^{c}$.

Prova: Uma vez que $\mathrm{Y}_{W^{\prime}}=\mathrm{X}_{W} \oplus C^{t}=\sqcup\left\{\left(\mathrm{X}_{h}\right)_{W \oplus C^{t}}: h \in C^{t}\right\}$, então $W^{\prime}=$ $W \oplus C^{t}$ e $\mathbf{Y}_{W^{\prime}}$ é o supremo de $\left(\mathbf{X}_{h}\right)_{W \oplus C^{t}}, h \in C^{t}$. Assim, cada intervalo $\left[A^{\prime}, B^{\prime}\right] \in$ $\mathrm{Y}_{W^{\prime}}$ é construído pelo supremo de translações de intervalos em $\mathbf{X}_{W}$. Logo, $\mathbf{Y}_{W^{\prime}}=$ $\mathrm{M}\left(\cup\left\{\cup\left(\mathrm{X}_{h}\right)_{W \oplus C^{t}}: h \in C^{t}\right\}\right)$. Dessa forma, se $\left[A^{\prime}, B^{\prime}\right] \in \mathbf{Y}_{W^{\prime}}$, então existem dois pontos $h_{1}, h_{2} \in C^{t}$ e dois subconjuntos $A \in \mathcal{U}\left(\mathrm{X}_{h_{1}}\right)_{W \oplus C^{t}}$ e $B \in \mathcal{U}\left(\mathrm{X}_{h_{2}}\right)_{W \oplus C^{t}}$ tais que $A=A^{\prime}$ e $B=B^{\prime}$. Note que, se $A \in \mathcal{U}\left(\mathrm{X}_{h_{1}}\right)_{W \oplus C^{t}}$ e $A=A^{\prime}$ (respectivamente, $B \in \mathcal{U}\left(\mathrm{X}_{h_{2}}\right)_{W \oplus C^{t}}$ e $B=B^{\prime}$ ), então existe um intervalo $\left[L_{1}, R_{1}\right] \in X_{W}$ (respectivamente, um intervalo $\left.\left[L_{2}, R_{2}\right] \in X_{W}\right)$ tal que $\left(L_{1}\right)_{h_{1}}=A$ (respectivamente, $\left.\left(R_{2}\right)_{h_{2}}=B \cap W\right)$. Em outras palavras, para cada intervalo $\left[A^{\prime}, B^{\prime}\right] \in \mathrm{Y}_{W^{\prime}}$, existe um intervalo em $\mathrm{X}_{W}$ tal que sua translação contribui para gerar a extremidade esquerda do intervalo $\left[A^{\prime}, B^{\prime}\right]$. Da mesma forma, existe um outro intervalo em $\mathrm{X}_{W}$ tal que sua translação contribui para gerar a extremidade direita do intervalo $\left[A^{\prime}, B^{\prime}\right]$. Logo, para todo intervalo $\left[A^{\prime}, B^{\prime}\right] \in \mathrm{Y}_{W^{\prime}}$, existem dois intervalos $[P, Q],[X, Y] \in \mathrm{X}_{W}$ e dois pontos $a, b \in C^{t}$ tais que $P_{a}=A^{\prime}$ e $Y_{b} \cup\left(W_{b}\right)_{W^{\prime}}^{c}=B^{\prime}$. Assim, uma vez que $Y_{b} \subseteq W_{b}$, então, claramente, $Y_{b}=B^{\prime}-\left(W_{b}\right)_{W^{\prime}}^{c}$.

Agora, faremos duas definições para construir o conjunto $\Theta_{W^{\prime}, C}^{\mathbf{Y}_{W^{\prime}}}$. Primeiro, dado um intervalo $\left[A^{\prime}, B^{\prime}\right] \subseteq \mathcal{P}\left(W^{\prime}\right)$ e um conjunto $C \in \mathcal{P}(E)$, definiremos duas coleções de intervalos $\mathcal{L}_{W}^{\left[A^{\prime}, B^{\prime}\right], C}$ e $\mathcal{R}_{W}^{\left[A^{\prime}, B^{\prime}\right], C}$, contidos em $\mathcal{P}\left(\mathcal{P}(W)\right.$ ), onde $W=W^{\prime} \ominus C^{t}$. Com os intervalos destas duas coleções, podemos construir um conjunto de coleções de intervalos, denotada 
por $\mathcal{H}_{W}^{\left[A^{\prime}, B^{\prime}\right], C}$, e, finalmente, usando as coleções de intervalos em $\mathcal{H}_{W}^{\left[A^{\prime}, B^{\prime}\right], C}$ construiremos o conjunto de coleções de intervalos maximais $\Theta_{W}^{\mathbf{Y}_{W^{\prime}}, C}$. Após estas construções provaremos, no Teorema 9.8, que para toda solução $\mathbf{X}_{W}$ da Equação (1), existe uma coleção de intervalos maximais $\mathbf{Z}_{W} \in \Theta_{W}^{\mathbf{Y}_{W^{\prime}}, C}$ tal que $\mathbf{Z}_{W} \leq \mathbf{X}_{W}$.

Dado um intervalo $\left[A^{\prime}, B^{\prime}\right] \subseteq \mathcal{P}\left(W^{\prime}\right)$ e um conjunto $C \in \mathcal{P}(E)$, definimos as coleções de intervalos $\mathcal{L}_{W}^{\left[A^{\prime}, B^{\prime}\right], C}$ e $\mathcal{R}_{W}^{\left[A^{\prime}, B^{\prime}\right], C}$, contidos em $\mathcal{P}(\mathcal{P}(W))$, onde $W=W^{\prime} \ominus C^{t}$, como

$$
\mathcal{L}_{W}^{\left[A^{\prime}, B^{\prime}\right], C}=\left\{\left[A_{-x}^{\prime}, A_{-x}^{\prime}\right]: A^{\prime} \subseteq W_{x}, x \in C^{t}\right\}
$$

$\mathrm{e}$

$$
\mathcal{R}_{W}^{\left[A^{\prime}, B^{\prime}\right], C}=\left\{\left[B_{-x}^{\prime} \cap W, B_{-x}^{\prime} \cap W\right]: B^{\prime}-W_{x}=\left(W_{x}\right)_{W^{\prime}}^{c}, x \in C^{t}\right\} .
$$

Claramente, $\left|\mathcal{L}_{W}^{\left[A^{\prime}, B^{\prime}\right], C}\right| \leq|C|$ e $\left|\mathcal{R}_{W}^{\left[A^{\prime}, B^{\prime}\right], C}\right| \leq|C|$. A seguir, damos um exemplo de $\mathcal{L}_{W}^{\left[A^{\prime}, B^{\prime}\right], C}$ e $\mathcal{R}_{W}^{\left[A^{\prime}, B^{\prime}\right], C}$. Se $W^{\prime}=11 \underline{1} 11, C=0 \underline{1} 1$ e $\left[A^{\prime}, B^{\prime}\right]=[01 \underline{0000}, 11 \underline{101}]$, então $W=W^{\prime} \ominus C^{t}=$ $1 \underline{111}, \mathcal{L}_{W}^{\left[A^{\prime}, B^{\prime}\right], C}=\{[1 \underline{000}, 1 \underline{000}],[0 \underline{100}, 0 \underline{100}]\}$ e $\mathcal{R}_{W}^{\left[A^{\prime}, B^{\prime}\right], C}=\{[1 \underline{101}, 1 \underline{101}],[1 \underline{110}, 1 \underline{1} 10]\}$.

Agora, definimos o conjunto $\mathcal{H}_{W}^{\left[A^{\prime}, B^{\prime}\right], C}$ como

$$
\mathcal{H}_{W}^{\left[A^{\prime}, B^{\prime}\right], C}=\left\{\{[L, L]\} \sqcup\{[R, R]\}:[L, L] \in \mathcal{L}_{W}^{\left[A^{\prime}, B^{\prime}\right], C},[R, R] \in \mathcal{R}_{W}^{\left[A^{\prime}, B^{\prime}\right], C}\right\} .
$$

Claramente, $\left|\mathcal{H}_{W}^{\left[A^{\prime}, B^{\prime}\right], C}\right| \leq\left|\mathcal{L}_{W}^{\left[A^{\prime}, B^{\prime}\right], C}\right| \cdot\left|\mathcal{R}_{W}^{\left[A^{\prime}, B^{\prime}\right], C}\right| \leq|C|^{2}$. A seguir, damos um exemplo de $\mathcal{H}_{W}^{\left[A^{\prime}, B^{\prime}\right], C}$. Se $W^{\prime}=11 \underline{111}, C=1 \underline{11}$ e $\left[A^{\prime}, B^{\prime}\right]=[01 \underline{000}, 11 \underline{101}]$, então $W=W^{\prime} \ominus C^{t}=$ $1111 \mathrm{e}$

$$
\begin{aligned}
& \mathcal{H}_{W}^{\left[A^{\prime}, B^{\prime}\right], C}=\{\{[1 \underline{000}, 1 \underline{000}]\} \sqcup\{[1 \underline{1} 01,1 \underline{1} 01]\}, \\
& \{[1 \underline{000}, 1 \underline{000}]\} \sqcup\{[1 \underline{110}, 1 \underline{11} 0]\}, \\
& \{[0 \underline{100}, 0 \underline{100}]\} \sqcup\{[1 \underline{101}, 1 \underline{101}]\}, \\
& \{[0 \underline{100}, 0 \underline{100}]\} \sqcup\{[1 \underline{110}, 1 \underline{11} 10]\}\} . \\
& =\{\{[1 \underline{0} 00,1 \underline{0} 00],[1 \underline{101}, 1 \underline{101}]\}, \\
& \{[1 \underline{0} 00,1 \underline{000}],[1 \underline{1} 10,1 \underline{11} 10]\}, \\
& \{[0 \underline{100}, 0 \underline{100}],[1 \underline{101}, 1 \underline{101}]\} \text {, } \\
& \{[0 \underline{100}, 0 \underline{100}],][1 \underline{110}, 1 \underline{110}]\}\}
\end{aligned}
$$

Seja $I=\{1,2,3, \cdots, n\}$ um conjunto de índices. Sejam $\left.\mathbf{Y}_{W^{\prime}}=\left\{\left[A_{i}^{\prime}, B_{i}^{\prime}\right]: i \in I\right]\right\} \in$ $\Pi_{W^{\prime}}$ e $C \in \mathcal{P}(E)$. Definimos o conjunto

$$
\mathcal{S}_{W}^{\mathbf{Y}_{W^{\prime}}, C}=\mathcal{H}_{W}^{\left[A_{1}^{\prime}, B_{1}^{\prime}\right], C} \times \cdots \times \mathcal{H}_{W}^{\left[A_{n}^{\prime}, B_{n}^{\prime}\right], C} .
$$

Claramente, $\left|\mathcal{S}_{W^{W^{\prime}}, C}^{\mathbf{Y}}\right| \leq|C|^{2 n}$.

Dada uma coleção de intervalos maximais $\mathbf{Y}_{W^{\prime}} \in \Pi_{W^{\prime}}$ e um subconjunto $C \in \mathcal{P}(E)$, construiremos o conjunto de coleções de intervalos maximais $\Theta_{W}^{\mathbf{Y}_{W^{\prime}}, C}$, onde $W=W^{\prime} \ominus C^{t}$, como

$$
\Theta_{W}^{\mathbf{Y}_{W^{\prime}}, C}=\left\{\mathbf{Z}_{W} \in \Pi_{W}: \mathbf{Z}_{W}=\sqcup\left\{\mathbf{S}_{W}^{i}, i \in I\right\},\left(\mathbf{S}_{W}^{1}, \mathbf{S}_{W}^{2}, \cdots, \mathbf{S}_{W}^{n}\right) \in \mathcal{S}_{W}^{\mathbf{Y}_{W^{\prime}}, C}\right\} .
$$

Claramente, $\left|\Theta_{W}^{\mathbf{Y}_{W}, C}\right| \leq\left|\mathcal{S}_{W}^{\mathbf{Y}_{W^{\prime}}, C}\right| \leq|C|^{2 n}$. A seguir, damos um exemplo de $\Theta_{W}^{\mathbf{Y}_{W^{\prime}}, C}$. Sejam $W^{\prime}, C \in \mathcal{P}(E)$, os conjuntos apresentados nas Figuras 9.2.a e 9.2.b, respectivamente; seja 


$$
\begin{aligned}
& W^{\prime}=11 \underline{11} \quad C=\underline{011} \\
& \text { (a) (b) } \\
& {\left[A_{1}^{\prime}, B_{1}^{\prime}\right]=[00 \underline{1}, 01 \underline{1}] \quad\left[A_{2}^{\prime}, B_{2}^{\prime}\right]=[00 \underline{10} 0,01 \underline{11}] \quad\left[A_{3}^{\prime}, B_{3}^{\prime}\right]=[01 \underline{00}, 01 \underline{11}]} \\
& {\left[A_{4}^{\prime}, B_{4}^{\prime}\right]=[00 \underline{01}, 10 \underline{11}] \quad\left[A_{5}^{\prime}, B_{5}^{\prime}\right]=[00 \underline{10}, 10 \underline{11}] \quad\left[A_{6}^{\prime}, B_{6}^{\prime}\right]=[00 \underline{0} 1,11 \underline{01}]} \\
& {\left[A_{7}^{\prime}, B_{7}^{\prime}\right]=[01 \underline{00}, 11 \underline{01}] \quad\left[A_{8}^{\prime}, B_{8}^{\prime}\right]=[00 \underline{10}, 11 \underline{10}] \quad\left[A_{9}^{\prime}, B_{9}^{\prime}\right]=[01 \underline{00}, 11 \underline{10}]}
\end{aligned}
$$

(c)

$\mathbf{Y}_{W^{\prime}}=\left\{\left[A_{1}^{\prime}, B_{1}^{\prime}\right],\left[A_{2}^{\prime}, B_{2}^{\prime}\right],\left[A_{3}^{\prime}, B_{3}^{\prime}\right],\left[A_{4}^{\prime}, B_{4}^{\prime}\right],\left[A_{5}^{\prime}, B_{5}^{\prime}\right],\left[A_{6}^{\prime}, B_{6}^{\prime}\right],\left[A_{7}^{\prime}, B_{7}^{\prime}\right],\left[A_{8}^{\prime}, B_{8}^{\prime}\right],\left[A_{9}^{\prime}, B_{9}^{\prime}\right]\right\}$

(d)

$$
W=W^{\prime} \Theta C^{t}=1 \underline{1}
$$

(e)
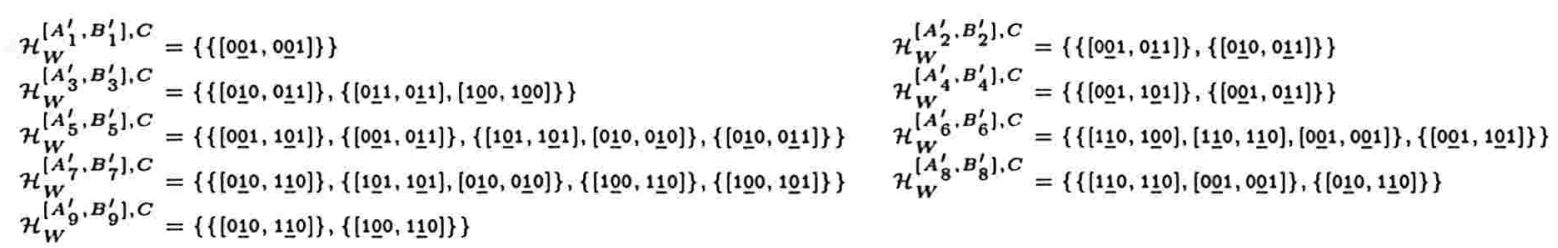

(f)

$$
S_{W}^{\mathbf{Y}_{W}}{ }^{\prime} \cdot C=\mathcal{H}_{W}^{\left[A_{1}^{\prime}, B_{1}^{\prime}\right], C} \times \cdots \times \mathcal{H}_{W}^{\left[A_{9}^{\prime}, B_{9}^{\prime}\right], C}
$$

$(a)$

$$
\begin{aligned}
& \mathbf{Z}_{1}=\{[\underline{100}, 1 \underline{1} 0],[0 \underline{0} 1,0 \underline{1} 1]\} \\
& \mathrm{Z}_{2}=\{[1 \underline{00}, 1 \underline{1} 0],[1 \underline{00}, 1 \underline{0} 1],[0 \underline{0} 1,1 \underline{0} 1],[0 \underline{01} 1,0 \underline{1} 1]\} \\
& Z_{3}=\{[1 \underline{00}, 1 \underline{1} 0],[0 \underline{10}, 1 \underline{1} 0],[0 \underline{10}, 0 \underline{11}],[0 \underline{01}, 0 \underline{11}]\} \\
& \mathbf{Z}_{4}=\{[0 \underline{10}, 1 \underline{10}],[0 \underline{0} 0,011],[0 \underline{01}, 0 \underline{11}]\}
\end{aligned}
$$

(h)

$$
\Theta_{W}^{\mathbf{Y}_{W^{\prime}}, C}=\left\{\mathbf{z}_{1}, \mathbf{z}_{2}, \cdots, \mathbf{z}_{6}\right\}
$$

(i)

Figura 9.2: Exemplo de $\mathcal{S}_{W}^{\mathbf{Y}_{W^{\prime}}, C}$ e $\Theta_{W}^{\mathbf{Y}_{W^{\prime}}, C}$.

$\mathrm{Y}_{W^{\prime}}$ a coleção de intervalos maximais apresentada na Figura 9.2.d. Note que $W^{\prime}, C$ e $\mathrm{Y}_{W^{\prime}}$ são exatamente o mesmos que foram apresentados nas Figuras 9.1.a, 9.1.b e 9.1.d, respectivamente. Assim, $\mathcal{S}_{W}^{\mathbf{Y}_{W^{\prime}}, C}=\mathcal{H}_{W}^{\left[A_{1}^{\prime}, B_{1}^{\prime}\right], C} \times \mathcal{H}_{W}^{\left[A_{2}^{\prime}, B_{2}^{\prime}\right], C} \times \cdots \times \mathcal{H}_{W}^{\left[A_{9}^{\prime}, B_{9}^{\prime}\right], C}$ e $\Theta_{W}^{\mathbf{Y}_{W^{\prime}}, C}=$ $\left\{\mathbf{Z}_{1}, \mathbf{Z}_{2}, \cdots, \mathbf{Z}_{6}\right\}$, onde $W=W^{\prime} \ominus C^{t}$ é o conjunto mostrado na Figura 9.2.c; $\mathcal{H}_{W}^{\left[A_{i}^{\prime}, B_{i}^{\prime}\right], C}$, para $i \in\{1,2, \cdots, 9\}$, são os conjuntos apresentados na Figura 9.2. $f$ e $\mathbf{Z}_{i}$, para $i \in\{1,2, \cdots, 6\}$, são as coleções de intervalos maximais de $\Pi_{W}$ mostradas na Figura 9.2.h.

O próximo resultado mostra quais são os limites inferiores para todas soluções $\mathbf{X}_{W}$ da Equação (1).

Teorema 9.8 Sejam $C \in \mathcal{P}(E)$ e $\mathbf{Y}_{W^{\prime}} \in \Pi_{W^{\prime}}$. Para todo $\mathbf{X}_{W} \in \Pi_{W}$ tal que $\mathbf{X}_{W} \oplus C^{t}=$ $\mathbf{Y}_{W^{\prime}}$, existe $\mathbf{Z}_{W} \in \Theta_{W}^{\mathbf{Y}_{W^{\prime}}, C}$ tal que $\mathbf{Z}_{W} \leq \mathbf{X}_{W}$.

Prova: Seja $\mathbf{Y}_{W^{\prime}}=\left\{\left[A_{i}^{\prime}, B_{i}^{\prime}\right]: i \in I\right\}$. Pela Proposição 9.7, para cada intervalo $\left[A_{i}^{\prime}, B_{i}^{\prime}\right] \in$ $\mathrm{Y}_{W^{\prime}}$, existem pontos $a_{i}, b_{i} \in C^{t}$ e intervalos $\left[P_{i}, Q_{i}\right],\left[X_{i}, Y_{i}\right] \in \mathrm{X}_{W}$ tais que $\left(P_{i}\right)_{a_{i}}=A_{i}^{\prime}$ e $\left(Y_{i}\right)_{b_{i}}=B_{i}^{\prime}-\left(W_{b_{i}}\right)_{W^{\prime}}^{c}$. Seja $\mathbf{S}_{W}^{i}=\left\{\left[P_{i}, P_{i}\right],\left[Y_{i}, Y_{i}\right]\right\}$. Seja $\mathbf{Z}_{W}$ a coleção de intervalos maximais de $\Pi_{W}$ tal que $\mathbf{Z}_{W}=\sqcup\left\{\mathbf{S}_{W}^{i}: i \in I\right\}$. 
Uma vez que $\left[P_{i}, P_{i}\right] \subseteq\left[P_{i}, Q_{i}\right]$ e $\left[Y_{i}, Y_{i}\right] \subseteq\left[X_{i}, Y_{i}\right]$, então temos que cada $\mathbf{S}_{W}^{i} \leq$ $\left\{\left[P_{i}, Q_{i}\right],\left[X_{i}, Y_{i}\right]\right\} \leq \mathbf{X}_{W}$. Assim, $\mathbf{Z}_{W}=\sqcup\left\{\mathbf{S}_{W}^{i}: i \in I\right\} \leq \mathbf{X}_{W}$.

Para provar que $\mathbf{Z}_{W} \in \Theta_{W}^{\mathbf{Y}_{W^{\prime}}, C}$, temos que mostrar que cada $\mathbf{S}_{W}^{i} \in \mathcal{H}_{W}^{\left[A_{i}^{\prime}, B_{i}^{\prime}\right], C}$. Para isso, devemos mostrar que $\left[P_{i}, P_{i}\right] \in \mathcal{L}_{W}^{\left[A_{i}^{\prime}, B_{i}^{\prime}\right], C}$ e $\left[Y_{i}, Y_{i}\right] \in \mathcal{R}_{W}^{\left[A_{i}^{\prime}, B_{i}^{\prime}\right], C}$.

Uma vez que $\left(P_{i}\right)_{a_{i}}=A_{i}^{\prime}$, então $\left(A_{i}^{\prime}\right)_{-a_{i}}=P_{i} \subseteq W$. Assim, $A_{i}^{\prime} \subseteq W_{a_{i}}$, e, conseqüentemente, $\left[P_{i}, P_{i}\right] \in \mathcal{L}_{W}^{\left[A_{i}^{\prime}, B_{i}^{\prime}\right], C}$.

Uma vez que $\left(Y_{i}\right)_{b_{i}}=B_{i}^{\prime}-\left(W_{b_{i}}\right)_{W^{\prime}}^{c}$ e $Y_{i} \subseteq W$, então, claramente, $Y_{i}=\left(B_{i}^{\prime}\right)_{-b_{i}} \cap W$. Portanto, $\left[Y_{i}, Y_{i}\right] \in \mathcal{R}_{W}^{\left[A_{i}^{\prime}, B_{i}^{\prime}\right], C}$.

Como uma conseqüência do Teorema 9.8, cada solução $\mathbf{X}_{W}$ da Equação (1) tem um limite inferior em $\Theta_{W}^{\mathbf{Y}_{W^{\prime}}, C}$. Mas, algumas coleções de intervalos maximais em $\Theta_{W}^{\mathbf{Y}_{W^{\prime}}, C}$ podem ser desconsideradas. Para o exemplo apresentado na Figura 9.2.h, somente os limites inferiores $\mathbf{Z}_{4}$ e $\mathbf{Z}_{6}$ são menores ou iguais ao limite superior $\mathbf{U}_{W}$ apresentado na Figura 9.1.i. Assim, neste particular exemplo, temos que $\mathbf{Z}_{i} \mathbb{Z} \mathbf{U}_{W}$, para $i \in\{1,2,3,5\}$, e conseqüentemente, eles não devem ser utilizados para encontrar as soluções da Equação (1). Agora, para as soluções apresentadas na Figura 9.1.j, temos que $\mathbf{Z}_{4} \leq \mathbf{X}_{1}, \mathbf{X}_{2} \leq \mathbf{U}_{W}$ e $\mathrm{Z}_{6} \leq \mathrm{X}_{1}, \mathrm{X}_{2} \leq \mathrm{U}_{W}$.

A seguir, apresentamos um algoritmo para encontrar todas soluções da Equação (1). Algoritmo SEARCH_1 $\left(C, \mathrm{Y}_{W^{\prime}}\right)$ :

Entrada: Um conjunto $C \in \mathcal{P}(E)$ e uma coleção de intervalos maximais $\mathrm{Y}_{W^{\prime}} \in \Pi_{W^{\prime}}$.

Saída: As coleções de intervalos maximais $\mathbf{X}_{W} \in \Pi_{W}$, tais que $\mathbf{X}_{W} \oplus C^{t}=\mathbf{Y}_{W^{\prime}}$, onde $W=W^{\prime} \ominus C^{t}$.

$W^{\prime \prime} \leftarrow\left(W \oplus C^{t}\right) \oplus C ;$

$\mathbf{U}_{W^{\prime \prime}} \leftarrow\left\{[L, R] \in \mathbf{Y}_{W^{\prime}} \ominus C^{t}:\{[L, R]\} \in \Pi_{W^{\prime \prime} / W}\right\}$.

para cada $\mathbf{Z}_{W} \in \Theta_{W}^{\mathbf{Y}_{W^{\prime}}, C}$ faça

para cada $\mathrm{X}_{W}$ tal que $\mathrm{Z}_{W} \leq \mathrm{X}_{W} \leq \mathrm{U}_{W}$ faça

se $\mathrm{X}_{W} \oplus C^{t}=\mathrm{Y}_{W^{\prime}}$ então

devolva $\mathbf{X}_{W}$.

Claramente, a cardinalidade do espaço de busca para o algoritmo SEARCH_1 $\left(C, \mathbf{Y}_{W^{\prime}}\right)$ é proporcional a $\left|\Theta_{W}^{\mathbf{Y}_{W^{\prime}}, C}\right| \leq|C|^{2 n}$, onde $n=\left|\mathbf{Y}_{W^{\prime}}\right|$, uma vez que cada limite inferior $\mathbf{Z}_{W}$ é retirado somente uma vez de $\Theta_{W}^{\mathbf{Y}_{W^{\prime}}, C}$. 


\subsection{Simplificação para Coleções de Extremidade Di- reita Fixa}

Definimos o conjunto $\mathcal{I}_{W} \subseteq \Pi_{W}$ como o conjunto de todas coleções de intervalos maximais tal que a extremidade direita de qualquer intervalo é a janela $W$. Chamaremos qualquer coleção de intervalos maximais de $\mathcal{I}_{W}$ por coleção de extremidade direita fixa.

Agora, considere o problema, apresentado na Seção 9.2 , restrita a $\mathcal{I}_{W}$ e $\mathcal{I}_{W^{\prime}}$, isto é, dada uma coleção de intervalos maximais $\mathrm{Y}_{W^{\prime}} \in \mathcal{I}_{W^{\prime}}$ e um conjunto $C \in \mathcal{P}(E)$, encontrar todas coleções de intervalos maximais $\mathrm{X}_{W} \in \mathcal{I}_{W}$ tais que $\mathrm{X}_{W} \oplus C^{t}=\mathbf{Y}_{W^{\prime}}$.

Por simplicidade de notação, onde não há risco de confusão, denotaremos os intervalos $[A, W]$ de $\mathrm{X} \in \mathcal{I}_{W}$ por $[A]$.

\subsubsection{Simplificação do Limite Inferior}

Podemos obter um novo limite inferior para $\mathrm{X}_{W} \in \mathcal{I}_{W}$, que é ele mesmo uma solução da Equação (1), restrita a coleções de extremidade direita fixa. Para isso, precisamos do seguinte resultado, que é um caso particular da Proposição 9.7, quando a extremidade direita dos intervalos em $\mathbf{Y}_{W^{\prime}}$ e $\mathbf{X}_{W}$ são, respectivamente, $W^{\prime}$ e $W$.

Proposição 9.9 Sejam $\mathbf{Y}_{W^{\prime}} \in \mathcal{I}_{W^{\prime}}, \mathbf{X}_{W} \in \mathcal{I}_{W}$ e $C \in \mathcal{P}(E)$. Se $\mathbf{Y}_{W^{\prime}}=\mathbf{X}_{W} \oplus C^{t}$, então, para cada intervalo $\left[A^{\prime}\right] \in \mathbf{Y}_{W^{\prime}}$, existe um intervalo $[P] \in \mathbf{X}_{W}$ e um ponto a $\in C^{t}$ tal que $P_{a}=A^{\prime}$.

Dado um intervalo $\left[A^{\prime}\right] \subseteq \mathcal{P}\left(W^{\prime}\right)$ e um subconjunto $C \in \mathcal{P}(E)$, definimos os conjuntos $\mathcal{L}_{W}^{\left[A^{\prime}\right], C}=\left\{\left[A_{-x}^{\prime}, W\right]: x \in C^{t}\right\}$ e $\mathcal{H}_{W}^{\left[A^{\prime}\right], C}=\left\{\{[P, W]\}:[P, W] \in \mathcal{L}_{W}^{\left[A^{\prime}\right], C}\right\}$. Claramente, $\left|\mathcal{H}_{W}^{\left[A^{\prime}\right], C}\right|=\left|\mathcal{L}_{W}^{\left[A^{\prime}\right], C}\right| \leq|C|$. A seguir, damos um exemplo de $\mathcal{L}_{W}^{\left[A^{\prime}\right], C}$ e $\mathcal{H}_{W}^{\left[A^{\prime}\right], C}$. Sejam $W^{\prime}$ e $C$ os conjuntos apresentados nas Figuras 9.3. $a$ e 9.3.b, respectivamente. Neste caso, $W=W^{\prime} \ominus C^{t}$ é o conjunto mostrado na Figura 9.3.e. Se $\left[A_{1}^{\prime}\right],\left[A_{2}^{\prime}\right],\left[A_{3}^{\prime}\right]$ são os intervalos apresentados na Figura 9.3.c, então os conjuntos $\mathcal{L}_{W}^{\left[A_{1}^{\prime}\right], C}, \mathcal{L}_{W}^{\left[A_{2}^{\prime}\right], C}$ e $\mathcal{L}_{W}^{\left[A_{3}^{\prime}\right], C}$ são aqueles que estão apresentados na Figura 9.3.f e os conjuntos $\mathcal{H}_{W}^{\left[A_{1}^{\prime}\right], C}, \mathcal{H}_{W}^{\left[A_{2}^{\prime}\right], C}$ e $\mathcal{H}_{W}^{\left[A_{3}^{\prime}\right], C}$ são aqueles que estão mostrados na Figura 9.3.g.

Seja $I=\{1,2,3, \cdots, n\}$ um conjunto de índices. Seja $\left.\mathrm{Y}_{W^{\prime}}=\left\{\left[A_{i}^{\prime}\right]: i \in I\right]\right\} \in \mathcal{I}_{W^{\prime}}$ e $C \in \mathcal{P}(E)$. Definimos o conjunto $\mathcal{F}_{W^{W^{\prime}}, C}^{\mathbf{Y}}=\mathcal{H}_{W}^{\left[A_{1}^{\prime}\right], C} \times \cdots \times \mathcal{H}_{W}^{\left[A_{n}^{\prime}\right], C}$. Claramente, $\left|\mathcal{F}_{W}^{Y_{W^{\prime}}, C}\right| \leq|C|^{n}$. A seguir, damos um exemplo de $\mathcal{F}_{W}^{\mathrm{Y}_{W^{\prime}}, C}$. Se $\mathrm{Y}_{W^{\prime}}$ e $C$ são a coleção de intervalos maximais e o conjunto apresentados nas Figuras 9.3.d e 9.3.b, respectivamente, então o conjunto $\mathcal{F}_{W^{W^{\prime}}, C}^{\mathbf{Y}}$ é aquele que está mostrado na Figura Figure 9.3.h.

Dada uma coleção de intervalos maximais $\mathbf{Y}_{W^{\prime}} \in \mathcal{I}_{W^{\prime}}$ e um subconjunto $C \in \mathcal{P}(E)$, definimos o conjunto de coleção de intervalos maximais $\Phi_{W^{\prime}}^{\mathrm{Y}^{\prime}, C}$, onde $W=W^{\prime} \ominus C^{t}$, como 


$$
\begin{aligned}
& W^{\prime}=11 \underline{11} \quad C=1 \underline{11} \\
& \text { (a) (b) } \\
& {\left[A_{1}^{\prime}\right]=[00 \underline{10} 0] \quad\left[A_{2}^{\prime}\right]=[00 \underline{100}] \quad\left[A_{3}^{\prime}\right]=[01000]} \\
& \text { (c) } \\
& \mathbf{Y}_{W^{\prime}}=\left\{\left[A_{1}^{\prime}\right],\left[A_{2}^{\prime}\right],\left[A_{3}^{\prime}\right]\right\} \\
& \text { (d) } \\
& W=W^{\prime} \theta C^{t}=1 \underline{11} \\
& \text { (e) } \\
& \mathcal{L}_{W}^{\left[A_{1}^{\prime}\right], C}=\{[0 \underline{10}],[0 \underline{0} 1]\} \quad \mathcal{L}_{W}^{\left[A_{2}^{\prime}\right], C}=\{[100],[010],[001]\} \quad \mathcal{L}_{W}^{\left[A_{3}^{\prime}\right], C}=\{[100],[010]\} \\
& \text { (f) } \\
& \mathcal{H}_{W}^{\left[A_{1}^{\prime}\right], C}=\{\{[0 \underline{10}]\},\{[0 \underline{0} 1]\}\} \quad \mathcal{H}_{W}^{\left[A_{2}^{\prime}\right], C}=\{\{[\underline{100}]\},\{[\underline{010}]\},\{[0 \underline{0} 1]\}\} \quad \mathcal{H}_{W}^{\left[A_{3}^{\prime}\right], C}=\{\{[1 \underline{0} 0]\},\{[0 \underline{10}]\}\} \\
& \text { (g) } \\
& \mathcal{F}_{W}^{Y_{W}^{\prime}, C}=\mathcal{H}_{W}^{\left[A_{1}^{\prime}\right], C} \times \mathcal{H}_{W}^{\left[A_{2}^{\prime}\right], C} \times \mathcal{H}_{W}^{\left[A_{3}^{\prime}\right], C} \\
& \mathrm{Z}_{1}=\{[\underline{001}],[100]\} \\
& \mathrm{Z}_{2}=\{[0 \underline{0} 1],[0 \underline{0} 0]\} \\
& \mathrm{z}_{3}=\{[100],[010],[001]\} \\
& \mathbf{Z}_{4}=\{[0 \underline{10} 0]\} \\
& \mathbf{Z}_{5}=\{[1 \underline{00}],[010]\} \\
& { }_{\Phi_{W}}^{\mathbf{Y}_{W^{\prime}}, C}=\left\{\mathbf{z}_{1}, \mathbf{z}_{2}, \cdots, \mathbf{z}_{5}\right\}
\end{aligned}
$$

(j)

Figura 9.3: Exemplo de $\mathcal{F}_{W}^{\mathbf{Y}_{W^{\prime}}, C}$ e $\Phi_{W}^{\mathbf{Y}_{W^{\prime}}, C}$.

$\Phi_{W^{W^{\prime}}, C}^{\mathbf{Y}}=\left\{\mathbf{Z}_{W} \in \mathcal{I}_{W}: \mathbf{Z}_{W}=\sqcup\left\{\mathbf{F}_{W}^{i}, i \in I\right\},\left(\mathbf{F}_{W}^{1}, \mathbf{F}_{W}^{2}, \cdots, \mathbf{F}_{W}^{n}\right) \in \mathcal{F}_{W}^{\mathbf{Y}_{W^{\prime}}, C}\right\}$. Claramente, $\left|\Phi_{W}^{\mathbf{Y}_{W^{\prime}}, C}\right| \leq\left|\mathcal{F}_{W^{\prime}}^{\mathbf{Y}_{W^{\prime}}, C}\right| \leq|C|^{n}$. Um exemplo de $\Phi_{W}^{\mathbf{Y}_{W^{\prime}}, C}$ pode ser visto na Figura 9.3.j.

Os limites superiores $\mathbf{U}_{W^{\prime \prime}}$ para $\mathbf{X}_{W^{\prime \prime}}$ e $\mathbf{U}_{W}$ para $\mathbf{X}_{W}$ estão apresentados nas Figuras 9.4.g e 9.4. $h$, respectivamente. Note que somente dois intervalos de $\mathrm{Y}_{W^{\prime}} \ominus C^{t}$ (apresentados na Figura 9.4.f) aparecem em $\mathbf{U}_{W^{\prime \prime}}$. A Figura 9.4.i apresenta duas soluções da Equação (1). Além disso, para este exemplo, somente o limite inferior $\mathbf{Z}_{4}$ é menor ou igual ao limite superior $\mathbf{U}_{W}$.

O próximo resultado mostra os limites inferiores para as soluções $\mathbf{X}_{W}$ da Equação (1) para coleções de extremidade direita fixa.

Teorema 9.10 Sejam $C \in \mathcal{P}(E)$ e $\mathbf{Y}_{W^{\prime}} \in \mathcal{I}_{W^{\prime}}$. Para todo $\mathbf{X}_{W} \in \mathcal{I}_{W}$ tal que $\mathbf{X}_{W} \oplus C^{t}=$ $\mathbf{Y}_{W^{\prime}}$, existe $\mathbf{Z}_{W} \in \Phi_{W}^{\mathbf{Y}_{W^{\prime}}, C}$ tal que $\mathbf{Z}_{W} \leq \mathbf{X}_{W}$ e $\mathbf{Z}_{W} \oplus C^{t}=\mathbf{Y}_{W^{\prime}}$.

Prova: Seja $\mathbf{Y}_{W^{\prime}}=\left\{\left[A_{i}^{\prime}\right]: i \in I\right\}$. Pela Proposição 9.9, para cada intervalo $\left[A_{i}^{\prime}\right] \in \mathbf{Y}_{W^{\prime}}$, existem $a_{i} \in C^{t}$ e $\left[P_{i}\right] \in \mathbf{X}_{W}$ tais que $\left(P_{i}\right)_{a_{i}}=A_{i}^{\prime}$. Seja $\mathbf{F}_{W}^{i}=\left\{\left[P_{i}\right]\right\}$, para $i \in I$. Seja $\mathbf{Z}_{W}$ a coleção de intervalos maximais de $\mathcal{I}_{W}$ tal que $\mathbf{Z}_{W}=\sqcup\left\{\mathbf{F}_{W}^{i}: i \in I\right\}$.

A prova de que $\mathbf{Z}_{W} \in \Phi_{W}^{\mathbf{Y}_{W^{\prime}}, C}$ e $\mathbf{Z}_{W} \leq \mathbf{X}_{W}$ pode ser feita de uma maneira similar ao do Teorema 9.8 .

Agora, provaremos que $\mathbf{Z}_{W} \oplus C^{t}=\mathbf{Y}_{W^{\prime}}$. Para isso, dividiremos esta prova em duas partes. Na primeira, provaremos que $\mathbf{Z}_{W} \oplus C^{t} \leq \mathrm{Y}_{W^{\prime}}$ e, na segunda, mostraremos $\mathbf{Y}_{W^{\prime}} \leq$ 


$$
\begin{aligned}
& W^{\prime}=11 \underline{11} \quad C=1 \underline{11} \\
& \text { (a) (b) } \\
& W=W^{\prime} \ominus C^{t}=1 \underline{11} \quad W^{\prime \prime}=\left(W \oplus C^{t}\right) \oplus C=111 \underline{1111} \\
& \text { (d) } \\
& \mathrm{Y}_{W^{\prime}} \ominus C^{t}=\{[0001000],[0010010],[0010000],[0100100]\} \\
& \text { (f) } \\
& U_{W^{\prime \prime}}=\{\{[0001000],[0010100]\} \\
& \text { (g) } \\
& \mathbf{U}_{W}=\{[010],[101]\} \\
& \text { (h) } \\
& \mathbf{X}_{1}=\{[1 \underline{01} 1],[0 \underline{10} 0]\} \\
& \mathrm{X}_{2}=\{[010]\} \\
& \text { (i) }
\end{aligned}
$$

Figura 9.4: Limite superior e as soluções para o exemplo apresentado na Figura 9.3.

$\mathrm{Z}_{W} \oplus C^{t}$

Uma vez que $\mathbf{Z}_{W} \leq \mathbf{X}_{W}$, então, pela Proposição 9.3 , podemos ver facilmente que $\mathrm{Z}_{W} \oplus C^{t} \leq \mathrm{X}_{W} \oplus C^{t}=\mathrm{Y}_{W^{\prime}}$.

Observe que $\left\{\left[\left(A_{i}^{\prime}\right)_{-a_{i}}, W\right]\right\} \oplus C^{t} \in \mathcal{I}_{W^{\prime}}$. Além disso, temos que $\left\{\left[\left(A_{i}^{\prime}\right)_{-a_{i}}, W_{-a_{i}}^{\prime}\right]_{a_{i}}\right\} \leq$ $\left\{\left[\left(A_{i}^{\prime}\right)_{-a_{i}}, W\right]\right\} \oplus C^{t}$, uma vez que cada $a_{i} \in C^{t}$. Assim, podemos ver facilmente que $\mathrm{Y}_{W^{\prime}}=\left\{\left[A_{i}^{\prime}\right]: i \in I\right\}=\left\{\left[\left(A_{i}^{\prime}\right)_{-a_{i}}, W_{-a_{i}}^{\prime}\right]_{a_{i}}: i \in I\right\} \leq \sqcup\left\{\left\{\left[\left(A_{i}^{\prime}\right)_{-a_{i}}, W\right]\right\} \oplus C^{t}: i \in I\right\}$. Dessa forma, uma vez que $\mathbf{F}_{W}^{i}=\left\{\left[\left(A_{i}^{\prime}\right)_{-a_{i}}, W\right]\right\}$, temos que $\mathbf{Y}_{W^{\prime}} \leq \sqcup\left\{\mathbf{F}_{W}^{i} \oplus C^{t}: i \in I\right\}$. Conseqüentemente, pela Proposição 9.2, $\mathbf{Y}_{W^{\prime}} \leq \sqcup\left\{\mathbf{F}_{W}^{i}: i \in I\right\} \oplus \bar{C}^{t}=\mathbf{Z}_{W} \oplus C^{t}$.

Como conseqüência do Teorema 9.10 , todos limites inferiores para toda solução $\mathbf{X}_{W}$ da Equação (1), restrita a coleções de extremidades fixas, estão em $\Phi_{W}^{\mathbf{Y}_{W^{\prime}}, C}$. De fato, dada uma solução $\mathbf{X}_{W}$ da Equação (1), existe $\mathbf{Z}_{W} \in \Phi_{W}^{\mathbf{Y}_{W^{\prime}}, C}$ tal que $\mathbf{Z}_{W} \oplus C^{t}=\mathrm{Y}_{W^{\prime}}$ e $\mathrm{Z}_{W} \leq \mathrm{X}_{W}$.

Apresentamos agora uma algoritmo para encontrar todas soluções da Equação (1), onde $\mathrm{Y}_{W^{\prime}} \in \mathcal{I}_{W^{\prime}}$.

Algoritmo SEARCH_2 $\left(C, \mathrm{Y}_{W^{\prime}}\right)$ :

Entrada: Um conjunto $C \in \mathcal{P}(E)$ e uma coleção de intervalos maximais $\mathrm{Y}_{W^{\prime}} \in \mathcal{I}_{W^{\prime}}$.

Saída: As coleções de intervalos maximais $\mathrm{X}_{W} \in \mathcal{I}_{W}$, tais que $\mathrm{X}_{W} \oplus C^{t}=\mathrm{Y}_{W^{\prime}}$, onde $W=W^{\prime} \ominus C^{t}$.

$W^{\prime \prime} \leftarrow\left(W \oplus C^{t}\right) \oplus C ; \quad \mathbf{U}_{W^{\prime \prime}} \leftarrow\left\{[L] \in \mathbf{Y}_{W^{\prime}} \ominus C^{t}:\{[L]\} \in \mathcal{I}_{W^{\prime \prime} / W}\right\}$.

para cada $\mathbf{Z}_{W} \in \Phi_{W}^{\mathbf{Y}_{W^{\prime}}, C}$ faça

se $\mathrm{Z}_{W} \oplus C^{t}=\mathrm{Y}_{W^{\prime}}$ então

para cada $\mathrm{X}_{W}$ tal que $\mathrm{Z}_{W} \leq \mathrm{X}_{W} \leq \mathrm{U}_{W}$ faça

se $\mathrm{X}_{W} \oplus C^{t}=\mathrm{Y}_{W^{\prime}}$ então

devolva $\mathbf{X}_{W}$. 
Claramente, a cardinalidade do espaço de busca do algoritmo SEARCH_2 $\left(C, \mathrm{Y}_{W^{\prime}}\right)$ é proporcional a $\left|\Phi_{W}^{\mathbf{Y}_{W^{\prime}}, C}\right| \leq|C|^{n}$, onde $n=\left|\mathbf{Y}_{W^{\prime}}\right|$, uma vez que cada limite inferior $\mathbf{Z}_{W}$ é retirado somente uma vez de $\Phi_{W^{W^{\prime}}, C}^{\mathbf{Y}}$. Além disso, se usamos o algoritmo SEARCH_2 $\left(C, \mathbf{Y}_{W^{\prime}}\right)$, quando $\mathbf{Y}_{W^{\prime}} \in \mathcal{I}_{W^{\prime}} \subseteq \Pi_{W^{\prime}}$, em vez do algoritmo SEARCH_1 $\left(C, \mathbf{Y}_{W^{\prime}}\right)$, o espaço de busca para escolha dos limites inferiores é reduzido de $O\left(|C|^{2 n}\right)$ para $O\left(|C|^{n}\right)$.

\subsubsection{Conjuntos viáveis para $C$}

Na Equação (1), o subconjunto $C \in \mathcal{P}(E)$ é um parâmetro fixo. Obviamente, dada uma coleção $\mathbf{Y}_{W^{\prime}} \in \mathcal{I}_{W^{\prime}}$, podem existir alguns subconjuntos $C$ em $\mathcal{P}(E)$ tais que a Equação (1) não tenha solução. Por exemplo, se $W^{\prime}$ e $\mathbf{Y}_{W^{\prime}}$ são o subconjunto e a coleção de intervalos mostrados nas Figuras 9.3.a e 9.3.d, respectivamente, é fácil ver que para $C=111 \underline{111}$, a Equação (1) não tem solução, uma vez que $W^{\prime}=11111$, e, conseqüentemente, $W=$ $W^{\prime} \ominus C^{t}=\emptyset$.

Dada uma coleção de intervalos $\mathrm{Y}_{W^{\prime}} \in \mathcal{I}_{W^{\prime}}$, os subconjuntos $C$ em $\mathcal{P}(E)$ tais que a Equação (1) tem pelo menos uma solução são chamados de conjuntos viáveis. Por exemplo, se $\mathbf{Y}_{W^{\prime}}$ é a coleção de intervalos apresentada na Figura 9.3.d, então o conjunto $C$ mostrado na Figura 9.3.b é viável.

Nesta subseção, estudaremos algumas propriedades da Equação (1) para dar algumas condições necessárias para a existência de conjuntos viáveis. Observe que, pela Proposição 9.4 , se um conjunto $C \in \mathcal{P}(E)$ é viável, então, para todo $h \in E$, o conjunto $C_{h}$ também é viável.

Seja $[A] \in \mathbf{X} \in \mathcal{I}_{W}$. Dizemos que a extremidade esquerda $A$ é minimal em $\mathbf{X}$ se, e somente se, $|A| \leq|B|$, para todo intervalo $[B] \in \mathbf{X}$. Claramente, se $|A|=|B|$, então tanto a extremidade esquerda de $[A]$ como a de $[B]$ são minimais. Por exemplo, sejam let $W=11 \underline{111}, A_{1}=00 \underline{101}, A_{2}=10 \underline{100}, A_{3}=01 \underline{000}, A_{4}=00 \underline{100}, A_{5}=00 \underline{0} 10 \mathrm{e}$ $\mathbf{A}_{W}=\left\{\left[A_{1}\right],\left[A_{2}\right],\left[A_{3}\right],\left[A_{4}\right],\left[A_{5}\right]\right\}$, então os intervalos $\left[A_{3}\right],\left[A_{4}\right]$ e $\left[A_{5}\right]$ têm extremidade esquerda minimal em $\mathbf{A}_{W}$.

Seja $\mathbf{Z} \in \mathcal{I}_{W}$. Denotaremos por $\operatorname{Min}(\mathbf{Z})$ o conjunto de todos intervalos em $\mathbf{Z}$ tais que suas extremidades esquerdas são minimais em $\mathbf{Z}$, ou $\operatorname{seja}, \operatorname{Min}(\mathbf{Z})=\{[A] \in \mathbf{Z}$ : $A$ é minimal em $\mathbf{Z}\}$. Para o último exemplo, $\operatorname{Min}(\mathbf{A})=\left\{\left[A_{3}\right],\left[A_{4}\right],\left[A_{5}\right]\right\}$.

Dada uma coleção de intervalos maximais $\mathbf{Z} \in \mathcal{I}_{W}$, para cada conjunto $A \in \mathcal{P}(E)$, definimos o conjunto $S_{A}^{\mathbf{Z}} \in \mathcal{P}(E)$ como $S_{A}^{\mathbf{Z}}=\left\{h \in E:\left[A_{-h}\right] \in \mathbf{Z}\right\}$. Para o último exemplo,

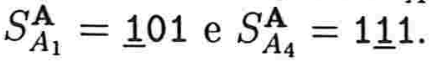

O próximo resultado fornece uma condição necessária para conjuntos viáveis.

Teorema 9.11 Sejam $\mathrm{Y}_{W^{\prime}} \in \mathcal{I}_{W^{\prime}}$ e $C \in \mathcal{P}(E)$. Se $C$ é um conjunto viável, então, para todo $\left[A^{\prime}\right] \in \operatorname{Min}\left(\mathbf{Y}_{W^{\prime}}\right)$, existe $a \in E$ tal que $C_{a} \subseteq S_{A^{\prime}}^{\mathbf{Y}_{W^{\prime}}}$ e $C$ é um invariante de $S_{A^{\prime}}^{\mathbf{Y}_{W^{\prime}}}$.

Prova: Uma vez que $C$ é viável, existe $\mathrm{X}_{W} \in \mathcal{I}_{W}$ tal que $\mathrm{X}_{W} \oplus C^{t}=\mathrm{Y}_{W^{\prime}}$. Dado um 
intervalo $\left[A^{\prime}\right]$ em $\operatorname{Min}\left(\mathbf{Y}_{W^{\prime}}\right)$, denotamos por $\mathbf{I}_{A^{\prime}}^{\mathbf{X}_{W}} \subseteq \mathbf{X}_{W}$ e $\mathbf{I}_{A^{\prime}}^{\mathbf{Y}_{W^{\prime}}} \subseteq \mathbf{Y}_{W^{\prime}}$ as coleções de intervalos tais que $\mathbf{I}_{A^{\prime}}^{\mathbf{X}_{W}}=\left\{\left[A_{-x}^{\prime}\right]: x \in S_{A^{\prime}}^{\mathbf{X}_{W}}\right\}$ e $\mathbf{I}_{A^{\prime}}^{\mathbf{Y}_{W^{\prime}}}=\left\{\left[A_{-y}^{\prime}\right]: y \in S_{A^{\prime}}^{\mathbf{X}_{W^{\prime}}}\right\}$. Agora, provaremos que $S_{A^{\prime}}^{\mathbf{Y}_{W^{\prime}}}=S_{A^{\prime}}^{\mathbf{X}_{W}} \oplus C$.

Por um lado, $S_{A^{\prime}}^{\mathbf{X}_{W}} \oplus C \subseteq S_{A^{\prime}}^{\mathbf{Y}_{W^{\prime}}}$. De fato, seja $\left[A_{-x}^{\prime}\right] \in \mathbf{I}_{A^{\prime}}^{\mathbf{X}_{W}}$ e $c \in C^{t}$. Mostraremos que $\left[A_{-x+c}^{\prime}, W^{\prime}\right] \in \mathbf{Y}_{W^{\prime}}$. Suponha por absurdo que $\left[A_{-x+c}^{\prime}, W^{\prime}\right] \notin \mathbf{Y}_{W^{\prime}}$. Assim, existe um intervalo $\left[X^{\prime}, W^{\prime}\right] \in \mathbf{Y}_{W^{\prime}}$ tal que $X^{\prime} \subseteq A_{-x+c}^{\prime}$ e $X^{\prime} \neq A_{-x+c}^{\prime}$. Assim, pela Proposição 2.1, $\left|X^{\prime}\right|<\left|A_{-x+c}^{\prime}\right|$. Mas, isto é uma contratriz o fato de que $A^{\prime}$ é minimal em $\mathbf{Y}_{W^{\prime}}$. Dessa forma, $\left[A_{-x+c}^{\prime}, W^{\prime}\right] \in \mathbf{Y}_{W^{\prime}}$. Logo, existe $y \in S_{A^{\prime}}^{\mathbf{Y}^{\prime}}$ tal que $\left[A_{-x+c}^{\prime}\right]=\left[A_{-y}^{\prime}\right]$, ou seja, $-x+c=-y$. Assim, $x+(-c)=y$ e , portanto $S_{A^{\prime}}^{\mathbf{X}_{W}} \oplus C \subseteq S_{A^{\prime}}^{\mathbf{Y}_{W^{\prime}}}$, uma vez que $c \in C^{t}$.

Por outro lado, $S_{A^{\prime}}^{\mathbf{Y}_{W^{\prime}}} \subseteq S_{A^{\prime}}^{\mathbf{X}_{W}} \oplus C$. Para provar isso, temos que mostrar que, dado um intervalo $\left[A_{-y}^{\prime}\right] \in \mathbf{Y}_{W^{\prime}}$, então existem $x \in S_{A^{\prime}}^{\mathbf{X}_{W}}$ e $c \in C^{t}$ tais que $-y=-x+c$. Pela Proposição 9.9, existem um intervalo $[P] \in \mathrm{X}_{W}$ e um ponto $c \in C^{t}$ tais que $P_{c}=A_{-y}^{\prime}$. Assim, $[P]=\left[A_{-c-y}^{\prime}\right]$ e, conseqüentemente, $[P] \in \mathbf{I}_{A^{\prime}}^{\mathbf{X}_{W}}$. Dessa forma, existe $x \in S_{A^{\prime}}^{\mathbf{X}_{W}}$ tal que $[P]=\left[A_{-x}^{\prime}\right]$. Dessa forma, $-y=-x+c$. Portanto, $y=x+(-c)$, e, uma vez que $y \in S_{A^{\prime}}^{\mathbf{Y}_{W^{\prime}}}, x \in S_{A^{\prime}}^{\mathbf{X}_{W}}$ e $c \in C^{t}$, então $S_{A^{\prime}}^{\mathbf{Y}_{W^{\prime}}} \subseteq S_{A^{\prime}}^{\mathbf{X}_{W}} \oplus C$.

Uma vez que $S_{A^{\prime}}^{\mathbf{Y}_{W^{\prime}}}=S_{A^{\prime}}^{\mathbf{X}_{W}} \oplus C$, então, pela Proposição 3.15, $C$ é um invariante de $S_{A^{\prime}}^{\mathbf{Y}_{W^{\prime}}}$. Falta ainda mostrar que existe $a \in E$ tal que $C_{a} \subseteq S_{A^{\prime}}^{\mathbf{Y}_{W^{\prime}}}$.

Seja $a \in S_{A^{\prime}}^{\mathbf{X}_{W}}$. Pela Proposição 3.10 e pela definição de dilatação, temos que $S_{A^{\prime}}^{\mathbf{Y}_{W^{\prime}}}=$ $\left(S_{A^{\prime}}^{\mathbf{X}_{W}}\right)_{-a} \oplus C_{a}$. Uma vez que $a \in S_{A^{\prime}}^{\mathbf{X}_{W}}$, então $o \in\left(S_{A^{\prime}}^{\mathbf{X}_{W}}\right)_{-a}$, e, pela definição e pela propriedade de comutatividade da adição de Minkowski, $C_{a} \subseteq C_{a} \oplus\left(S_{A^{\prime}}^{\mathbf{X}_{W}}\right)_{-a}=\left(S_{A^{\prime}}^{\mathbf{X}_{W}}\right)_{-a} \oplus$ $C_{a}=S_{A^{\prime}}^{\mathbf{Y}_{W^{\prime}}}$.

Devemos observar que, dada uma coleção de intervalos maximais $\mathbf{Y}_{W^{\prime}} \in \mathcal{I}_{W^{\prime}}$, como uma conseqüência da Proposição 9.4, se $C$ é uma solução viável, então, para todo $h \in E$, $C_{h}$ também o é. Assim, para encontrar todos conjuntos viáveis $C_{h}$, é suficiente procurar somente um conjunto viável $C$ que represente todos os conjuntos viáveis $C_{h}$. Chamaremos o conjunto viável $C$ que representa todas as suas translações por representante do conjunto viável.

Dada uma coleção de intervalos maximais $\mathrm{Y}_{W^{\prime}} \in \mathcal{I}_{W^{\prime}}$, pelo Teorema 9.11, se $C$ é viável, então, para todo $\left[A^{\prime}\right] \in \operatorname{Min}\left(\mathbf{Y}_{W^{\prime}}\right)$, uma translação de $C$, digamos $C_{a}$, é um subconjunto de $S_{A^{\prime}}^{\mathbf{Y}_{W^{\prime}}}$. Pela última observação, $C_{a}$ é também um conjunto viável. Portanto, podemos encontrar todos os representantes dos conjuntos viáveis $C$ procurando $C \subseteq S_{A^{\prime}}^{\mathbf{Y}_{W^{\prime}}}$ tal que $C$ é um invariante de $S_{A^{\prime}}^{\mathbf{Y}^{\prime}}$.

Agora, dada uma coleção de intervalos maximais $\mathrm{Y}_{W^{\prime}} \in \mathcal{I}_{W^{\prime}}$, apresentamos o algoritmo SeArch_All que tem como saída pares $\left(C, \mathrm{X}_{W}\right) \in \mathcal{P}(E) \times \mathcal{I}_{W}$ tais que $\mathrm{X}_{W} \oplus C^{t}=\mathrm{Y}_{W^{\prime}}$. Algoritmo SEARCh_AlL $\left(\mathbf{Y}_{W^{\prime}}\right)$ : 
Entrada: Uma coleção de intervalos maximais $\mathrm{Y}_{W^{\prime}} \in \mathcal{I}_{W^{\prime}}$.

Saída: $\quad$ Pares $\left(C, \mathrm{X}_{W}\right) \in \mathcal{P}(E) \times \mathcal{I}_{W}$, tais que $\mathrm{X}_{W} \oplus C^{t}=\mathrm{Y}_{W^{\prime}}$, com $W=W^{\prime} \ominus C^{t}$.

Seja $\left[A^{\prime}\right] \in \operatorname{Min}\left(\mathbf{Y}_{W^{\prime}}\right)$ tal que, para todo $\left[D^{\prime}\right] \in \operatorname{Min}\left(\mathbf{Y}_{W^{\prime}}\right),\left|S_{A^{\prime}}^{\mathbf{Y}_{W^{\prime}}}\right| \leq\left|S_{D^{\prime}}^{\mathbf{Y}_{W^{\prime}}}\right|$.

para todo $C \subseteq S_{A^{\prime}}^{\mathbf{Y}^{\prime}}$ faça

se $C$ é um invariante de $S_{A^{\prime}}^{\mathbf{Y}^{\prime}}$ então

começo

seja $\left\{\mathrm{X}_{1}, \mathrm{X}_{2}, \cdots, \mathrm{X}_{n}\right\}$ a saída de SEARCH_2 $\left(C, \mathrm{Y}_{W^{\prime}}\right)$;

para cada $i=1,2, \cdots, n$ devolva o par $\left(C, \mathrm{X}_{i}\right)$;

fim.

Dada uma coleção de intervalos maximais $Y_{W^{\prime}} \in \mathcal{I}_{W^{\prime}}$, o algoritmo SEARCH_ALL $\left(\mathrm{Y}_{W^{\prime}}\right)$ procura os representantes dos conjuntos viáveis $C$ para a Equação (1) e, depois, ele chama SEARCH_2 $\left(C, \mathbf{Y}_{W^{\prime}}\right)$ para encontrar todas as soluções $\mathbf{X}_{W}$ da Equação (1). Em outras palavras, este algoritmo soluciona completamente a Equação (1) quando $\mathbf{Y}_{W^{\prime}} \in$ $\mathcal{I}_{W^{\prime}}$.

\subsection{Conversão da Sup-Decomposição para Decompo- sições Seqüenciais}

Denotaremos por $\Upsilon_{W}$ o conjunto de todos $W$-operadores que podem ser construídos por composições de erosões e dilatações. Note que o conjunto dos filtros alternados seqüenciais [43], LD's em uma janela $W$, é um subconjunto de $\Upsilon_{W}$. Dada uma base de um operador $\psi \in \Upsilon_{W}$, descrevemos nesta seção como encontrar uma representação seqüencial para $\psi$.

Vimos no Capítulo 3, na Subseção 3.6.1, que a base de um operador $\psi \in \Psi_{W}$ se simplifica no caso em que $\psi$ é crescente: a extremidade direita de todo intervalo na base de $\psi$ é a própria janela $W$, ou seja, $\psi$ é crescente se, e somente se, para todo intervalo $[A, B] \in \mathbf{B}_{W}(\psi)$, temos que $B=W$. Assim, podemos ver facilmente que, $\psi \in \Psi_{W}$ é crescente se, e somente se, $\mathbf{B}_{W}(\psi) \in \mathcal{I}_{W}$.

Note que se $\psi \in \Upsilon_{W}$, então $\psi$ é um operador crescente, uma vez que dilatações e erosões são operadores crescentes [24, p. 86] e composições de operadores crescentes geram operadores crescentes [24, p. 46]. Assim $\Upsilon_{W} \subseteq \mathcal{I}_{W}$. Além disso, se $\psi \in \Upsilon_{W}$, então $\psi^{*} \in \Upsilon_{W}$, uma vez que, pela Proposição 3.30, $\varepsilon_{C}^{*}=\delta_{C^{t}}$ e $\delta_{C}^{*}=\varepsilon_{C^{t}}$.

Se $\psi$ é um operador em $\Upsilon_{W}$, então uma representação de $\psi$ pode começar por uma dilatação ou uma erosão, ou seja, $\psi$ pode ser reescrito como $\delta_{C} \psi_{1}$ ou $\varepsilon_{C} \psi_{1}$, onde $C \in \mathcal{P}(E)$.

Proposição 9.12 Se $\psi \in \Upsilon_{W}$ e $C \in \mathcal{P}(E)$, então $\mathbf{B}_{W \oplus C}\left(\left(\varepsilon_{C^{t}} \psi\right)^{*}\right)=\mathbf{B}_{W}\left(\psi^{*}\right) \oplus C^{t}$. 


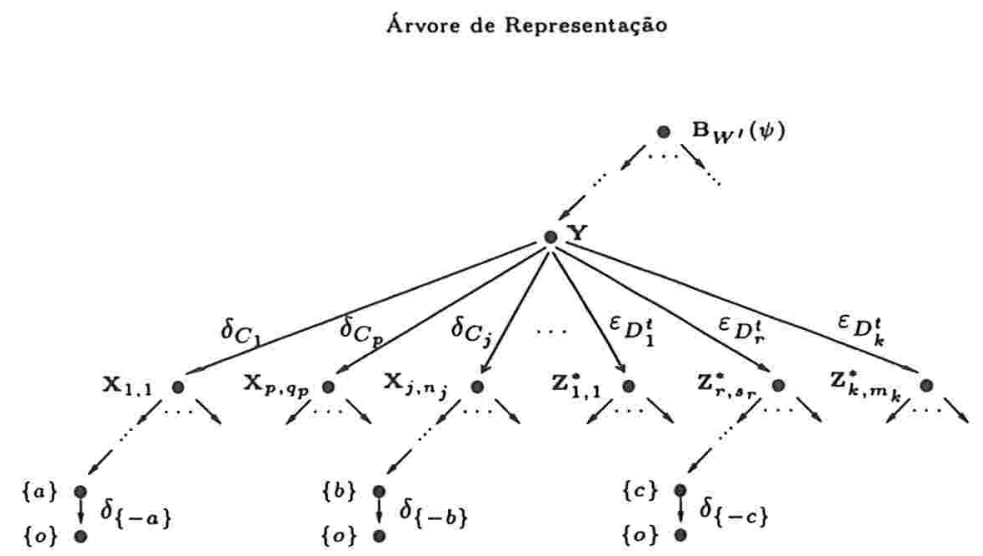

Figura 9.5: Um diagrama da árvore de representação.

Prova: Proposição $3.30, \varepsilon_{C^{t}}^{*}=\delta_{C}$ e, pela Proposição 3.41 , temos que $\mathrm{B}_{W \oplus C^{t}}\left(\delta_{C} \psi\right)=$ $\mathbf{B}_{W}(\psi) \oplus C^{t}$. Logo, $\mathbf{B}_{W \oplus C^{t}}\left(\left(\varepsilon_{C^{t}} \psi\right)^{*}\right)=\mathbf{B}_{W \oplus C^{t}}\left(\varepsilon_{C^{t}}^{*} \psi^{*}\right)=\mathbf{B}_{W \oplus C^{t}}\left(\delta_{C} \psi^{*}\right)=\mathbf{B}_{W}\left(\psi^{*}\right) \oplus C^{t}$.

Seja $\psi \in \Upsilon_{W}$. Como, pela Proposição 3.46, podemos construir a base de $\psi^{*}$ a partir da base de $\psi$, então, dada a base de um operador $\psi \in \Upsilon_{W}$ que começa por uma dilatação (respectivamente, erosão), podemos, pela Proposição 3.41 (respectivamente, Proposição 9.12), encontrar uma representação de $\psi$ aplicando o procedimento SEARCH_ALL para $\mathbf{B}_{W}(\psi)$ (respectivamente, para $\mathbf{B}_{W}\left(\psi^{*}\right)$ ). Se $(C, \mathbf{X})$ é uma saída do procedimento SEARCH_All $\left(\mathbf{B}_{W}(\psi)\right)$ (respectivamente, $\mathbf{B}_{W}\left(\psi^{*}\right)$ ), então $\psi$ pode ser reescrito como $\psi=\delta_{C} \psi_{1}$ (respectivamente, como $\left.\psi=\varepsilon_{C^{t}} \psi_{1}\right)$, onde $\psi_{1}$ é um $W$-operador crescente e $\mathbf{B}\left(\psi_{1}\right)=\mathbf{X}$ (respectivamente, $\mathbf{B}\left(\psi_{1}^{*}\right)=\mathbf{X}^{*}$ ).

Assim, dada a base de um operador $\psi \in \Upsilon_{W}$, podemos construir uma árvore que representa o espaço de busca de todas representações seqüenciais possíveis de $\psi$ da seguinte forma. A raiz é a base de $\psi$. Um nó é uma coleção de intervalos maximais $\mathrm{Y}_{W} \in \mathcal{I}_{W}$. Se $\mathrm{Y}_{W}=\{[\{o\}]\}$, então $\mathrm{Y}_{W}$ não tem filhos. Se $\mathbf{Y}_{W}=\{[\{a\}]\}, a \neq o$, então o filho de $\mathbf{Y}_{W}$ é $\{[\{o\}]\}$ e a aresta que liga $\mathrm{Y}_{W}$ e seus filhos é rotulada por $\delta_{\{-a\}}$. Caso contrário, construa $\mathrm{Y}_{W}^{*}$ usando o resultado da Proposição 3.46 e aplique o procedimento SEARCH_ALL para $\mathrm{Y}_{W}$ e $\mathrm{Y}_{W}^{*}$. Se $(C, \mathrm{X})$ é uma saída de SeArch_All $\left(\mathrm{Y}_{W}\right)$, então $\mathrm{X}$ é um filho de $\mathbf{Y}_{W}$ e a aresta que liga $\mathrm{Y}_{W}$ a $\mathrm{X}$ é rotulada por $\delta_{C}$. Se $(C, \mathrm{X})$ é uma saída do procedimento SEARCh_All $\left(\mathrm{Y}_{W}^{*}\right)$, então $\mathrm{X}^{*}$ é um filho de $\mathrm{Y}_{W}$ e a aresta que liga $\mathrm{Y}_{W}$ a $\mathrm{X}^{*}$ é rotulada por $\varepsilon_{C^{t}}$. Chamaremos esta árvore de árvore de representação.

Note que, dada a base de um operador $\psi \in \Upsilon_{W}$, os rótulos das arestas que estão no caminho da raiz a um nó $\mathrm{Y}_{W}=\{[\{o\}]\}$ forma uma representação seqüencial de $\psi$.

Na Figura 9.5, apresentamos um diagrama que descreve graficamente os filhos de um 
nó Y na árvore de representação. Para este diagrama, todas saídas de SEARCH_AlL(Y) são

$$
\begin{array}{cccc}
\left(C_{1}, \mathbf{X}_{1,1}\right) & \left(C_{1}, \mathbf{X}_{1,2}\right) & \cdots & \left(C_{1}, \mathbf{X}_{1, n_{1}}\right) \\
\left(C_{2}, \mathbf{X}_{2,1}\right) & \left(C_{2}, \mathbf{X}_{2,2}\right) & \cdots & \left(C_{2}, \mathbf{X}_{2, n_{2}}\right) \\
\vdots & \vdots & \vdots & \vdots \\
\left(C_{j}, \mathbf{X}_{j, 1}\right) & \left(C_{j}, \mathbf{X}_{j, 2}\right) & \cdots & \left(C_{j}, \mathbf{X}_{j, n_{j}}\right)
\end{array}
$$

e todas saídas de $\operatorname{SEARCH} \_\operatorname{ALL}\left(\mathrm{Y}^{*}\right)$ são

$$
\begin{array}{cccc}
\left(D_{1}, \mathbf{Z}_{1,1}\right) & \left(D_{1}, \mathbf{Z}_{1,2}\right) & \cdots & \left(D_{1}, \mathbf{Z}_{1, m_{1}}\right) \\
\left(D_{2}, \mathbf{Z}_{2,1}\right) & \left(D_{2}, \mathbf{Z}_{2,2}\right) & \cdots & \left(D_{2}, \mathbf{Z}_{2, m_{2}}\right) \\
\vdots & \vdots & \vdots & \vdots \\
\left(D_{k}, \mathbf{Z}_{k, 1}\right) & \left(D_{k}, \mathbf{Z}_{k, 2}\right) & \cdots & \left(D_{k}, \mathbf{Z}_{k, m_{k}}\right)
\end{array}
$$

Dessa forma, os filhos de $\mathrm{Y}$ são as coleções de intervalos maximais $\mathrm{X}_{p, q_{p}}$ e $\mathbf{Z}_{r, s_{r}}^{*}$, onde $p \in\{1,2, \cdots, j\}, q_{p} \in\left\{1,2, \cdots, n_{r}\right\}, r \in\{1,2, \cdots, k\}$ e $s_{r} \in\left\{1,2, \cdots, m_{t}\right\}$. A aresta que liga $\mathrm{Y}$ e $\mathrm{X}_{q, q_{p}}$ (respectivamente, $\mathbf{Z}_{r, s_{r}}^{*}$ ) é rotulada por $\delta_{C_{p}}$ (respectivamente, $\varepsilon_{D_{r}^{t}}$ ).

\subsection{O Algoritmo Implementado para Mudança de Es- trutura}

Implementamos os algoritmos SEARCh_1, SEARCh_2 e SEARCH_All apresentados nas Seções 9.2 e 9.3. Usando a implementação do algoritmo SEARCH_ALL, implementamos também um procedimento para transformar automaticamente a sup-decomposição de um dado operador $\psi \in \Upsilon_{W}$ em todas possíveis representações seqüenciais de $\psi$. A tarefa deste procedimento é visitar todos nós da árvore de representação aplicando o algoritmo SEARCH_All. Quando um nó $\{o\}$ é encontrado, o procedimento devolve uma representação de $\psi$ percorrendo e imprimindo os rótulos das arestas do caminho da raiz até esse nó. Existem muitas maneiras de visitar os nós de uma árvore. Para o algoritmo descrito acima, utilizamos a busca em profundidade [37, p. 39].

\subsection{Exemplos de Aplicação do Algoritmo}

Nesta seção, mostraremos algumas aplicações do método discutido na Seção 9.4.

Na Figura 9.6, mostramos um exemplo simples para encontrar uma representação seqüencial de um operador $\psi \in \Upsilon_{W^{\prime}}$. Neste exemplo, a base de $\psi$ é a coleção de intervalos maximais $\mathbf{Y}_{1}=\mathrm{Y}_{W^{\prime}}$ apresentada na Figura 9.6.a. A rais da árvore de representação é a base de entrada, ou seja, $\mathbf{Y}_{1}$. 

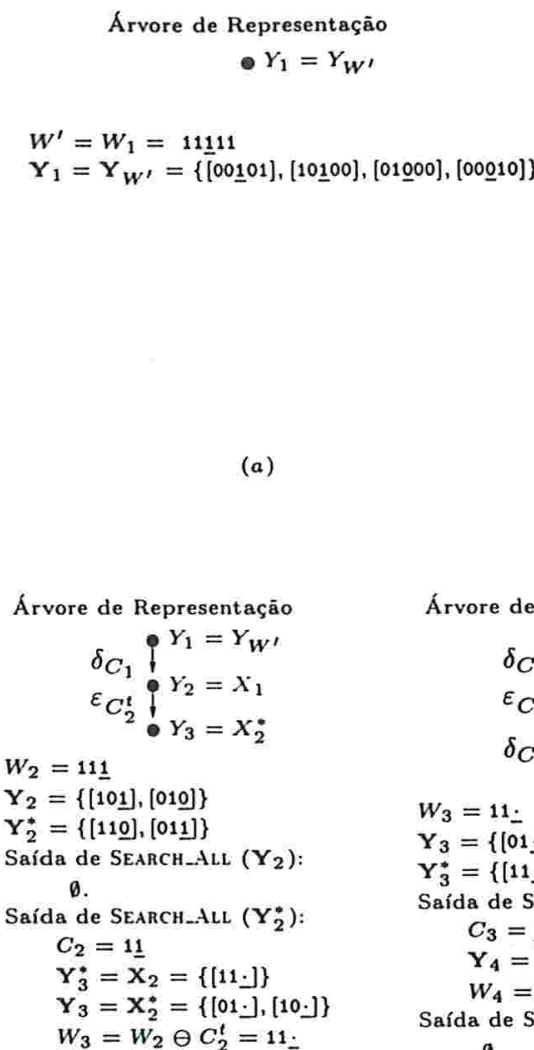

(c)

(a)
Árvore de Representação

$$
\delta_{C_{1}}\left\{\begin{array}{l}
Y_{1}=Y_{W^{\prime}} \\
Y_{2}=X_{1}
\end{array}\right.
$$

$W^{\prime}=W_{1}=11 \underline{111}$

$Y_{1}=Y_{W^{\prime}}=\{[00101],[10 \underline{100}],[01000],[00 \underline{010}]\}$

$\mathbf{Y}_{1}^{*}=\mathbf{Y}_{W^{\prime}}^{*}=\{[11 \underline{11}],[01 \underline{110}]\}$

Saída de SEARCh_All $\left(\mathbf{Y}_{1}\right)$ :

$C_{1}=10 \underline{1}$

$\mathrm{Y}_{2}=\mathrm{X}_{1}=\{[101],[010]\}$

$W_{2}=W^{\prime} \ominus C_{1}^{t}=111$

Saída de SEARCH_ALL ( $\left.Y_{i}^{*}\right)$ :

b.

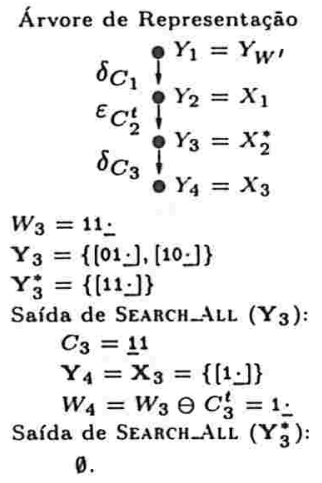

(d)
Figura 9.6: Applicação do método proposto para um exemplo simples.

A Figura 9.6.b mostra que as saídas do procedimento SEARCH_ALL quando aplicado a $\mathrm{Y}_{1}$ e $\mathrm{Y}_{1}^{*}$ são, respectivamente, $\left(C_{1}, \mathrm{X}_{1}\right)$ e $\emptyset$. Assim, na árvore de representação, o filho do nó raiz $\mathrm{Y}_{1}$ é $\mathrm{Y}_{2}=\mathrm{X}_{1}$ e a aresta que liga $\mathrm{Y}_{1}$ e $\mathrm{Y}_{2}$ é rotulada por $\delta_{C_{1}}$ (veja a Figura 9.6.b).

$\mathrm{Na}$ Figura 9.6.c, vemos que as saídas de SEARCH_AlL quando aplicado a $\mathrm{Y}_{2}$ e $\mathrm{Y}_{2}^{*}$ são, respectivamente, $\emptyset \mathrm{e}\left(C_{2}, \mathrm{X}_{2}\right)$. Assim, na árvore de representação, o filho do nó $\mathrm{Y}_{2}$ é $\mathrm{Y}_{3}=\mathrm{X}_{2}^{*}$ e a aresta que liga $\mathrm{Y}_{2}$ e $\mathrm{Y}_{3}$ é rotulada por $\varepsilon_{C_{2}^{t}}$, como mostrado na Figura 9.6.c.

Na Figura 9.6.d, mostramos que as saídas de SEARCH_AlL quando aplicado a $\mathrm{Y}_{3} \mathrm{e}$ $\mathrm{Y}_{3}^{*}$ são, respectivamente, $\left(C_{3}, \mathrm{X}_{3}\right)$ e $\emptyset$. Assim, na árvore de representação, o filho do nó $\mathrm{Y}_{3}$ é $\mathrm{Y}_{4}=\mathrm{X}_{3}$ e a aresta que liga $\mathrm{Y}_{3}$ e $\mathrm{Y}_{4}$ é rotulada por $\delta_{C_{3}}$, como mostra a Figura 9.6.d.

Finalmente, na Figura 9.6.e, $\mathrm{Y}_{4}=\{[\{a\}]\}$, onde $a=(-1,0)$. Uma vez que $a \neq o$, então o filho de $\mathrm{Y}_{4}$ é $\mathrm{Y}_{5}=\{[\{o\}]\}$ e a aresta que liga $\mathrm{Y}_{4}$ e $\mathrm{Y}_{5}$ é rotulada por $\delta_{C_{4}}$ com $C_{4}=\{-a\}$.

Os rótulos das arestas sobre o caminho da raiz até o nó $\mathrm{Y}_{5}$ forma uma representação seqüencial de $\psi$, ou seja, $\delta_{C_{1}} \varepsilon_{C_{2}^{t}} \delta_{C_{3}} \delta_{C_{4}}$ é uma representação seqüencial de $\psi$. Note que, 
para este exemplo, a sup-decomposição de $\psi$ tem 4 deslocamentos, 2 intersecções e 3 uniões. A representação seqüencial de $\psi$ tem 4 deslocamentos, 2 uniões e 1 intersecção. Assim, o número de deslocamentos, intersecções e uniões para implementar $\psi$ usando sua sup-decomposição é 9; se a representação seqüencial encontrada é usada, este número decresce para 7.

Como podemos ver neste exemplo simples, transformamos a sup-decomposição de $\psi$ (que tem uma estrutura paralela) para uma representação puramente seqüencial de $\psi$. A vantagem de representações seqüenciais sobre a sup-decomposição é que implementar representações seqüenciais é geralmente mais eficiente em máquinas convencionais seqüenciais.

A seguir damos alguns resultados experimentais da aplicação do algoritmo. Estes experimentos foram realizados usando uma máquina com processador Pentium III, $450 \mathrm{MHz}$. O tempo da CPU é medido em minutos (m) e segundos (s).

Sejam $W_{1}, W_{2}, W_{3}, W_{4}, W_{5}$ os subconjuntos apresentados nas Figuras 9.7.a, 9.7.d, 9.7.f, 9.7.h e 9.7.j, respectivamente. Sejam $\psi_{1}, \psi_{6} \in \Omega_{W_{1}} \psi_{2} \in \Omega_{W_{2}}, \psi_{3} \in \Omega_{W_{3}}, \psi_{4} \in \Omega_{W_{4}}$, $\psi_{5} \in \Omega_{W_{5}}$, tais que $\mathbf{B}_{W_{1}}\left(\psi_{1}\right)=\left\{\left[A_{1}\right], \cdots,\left[A_{8}\right]\right\}, \mathbf{B}_{W_{1}}\left(\psi_{6}\right)=\left\{\left[B_{1}\right], \cdots,\left[B_{9}\right]\right\}, \mathbf{B}_{W_{2}}\left(\psi_{2}\right)=$ $\left\{\left[D_{1}\right], \cdots,\left[D_{8}\right]\right\}, \mathbf{B}_{W_{3}}\left(\psi_{3}\right)=\left\{\left[F_{1}\right], \cdots,\left[F_{16}\right]\right\}, \mathbf{B}_{W_{3}}\left(\psi_{4}\right)=\left\{\left[G_{1}\right], \cdots,\left[G_{27}\right]\right\}$ e $\mathbf{B}_{W_{3}}\left(\psi_{5}\right)=$ $\left\{\left[H_{1}\right], \cdots,\left[H_{38}\right]\right\}$, onde $A_{1}, A_{2}, \cdots, A_{8}$ e $B_{1}, B_{2}, \cdots, B_{9}$ são os subconjuntos de $W_{1}$ apresentados nas Figuras 9.7.b e 9.7.c, respectivamente; $D_{1}, D_{2}, \cdots, D_{9}$ são os subconjuntos de $W_{2}$ apresentados na Figura 9.7.e; $F_{1}, F_{2}, \cdots, F_{16}$ são os subconjuntos de $W_{3}$ apresentados na Figura 9.7.g; $G_{1}, G_{2}, \cdots, G_{27}$ são os subconjuntos de $W_{4}$ apresentados na Figura 9.7.i e, finalmente, $H_{1}, H_{2}, \cdots, D_{38}$ são os subconjuntos de $W_{5}$ apresentados na Figura 9.7.k. Aplicando-se o método discutido na Seção 9.4, temos que as melhores representações seqüenciais de $\psi_{1}, \psi_{2}, \psi_{3}, \psi_{4}, \psi_{5}$ e $\psi_{6}$ são: $\psi_{1}=\delta_{C_{1}} \varepsilon_{C_{1}}, \psi_{2}=\delta_{C_{11}} \delta_{C_{12}} \varepsilon_{C_{11}} \varepsilon_{C_{12}}$, $\psi_{3}=\varepsilon_{C_{2}} \delta_{C_{3}} \varepsilon_{C_{3}} \psi_{4}=\delta_{C_{4}} \varepsilon_{C_{5}} \delta_{C_{5}}, \psi_{5}=\delta_{C_{6}} \varepsilon_{C_{5}} \delta_{C_{5}}, \psi_{6}=\delta_{C_{7}} \delta_{C_{8}} \delta_{C_{9}} \delta_{C_{10}} \varepsilon_{C_{7}} \varepsilon_{C_{8}} \varepsilon_{C_{9}} \varepsilon_{C_{10}}$, onde $C_{1}, C_{2}, \cdots, C_{12}$ são os conjuntos apresentados na Figura 9.7.l.

A Tabela 9.1 resume os resultados experimentais. Nesta tabela, usamos a seguinte notação:

NSD: número de EE's na sup-decomposição;

OSD: número de deslocamentos, intersecções e uniões usando a sup-decomposição;

NS: número de soluções encontradas pelo algoritmo;

OBS: número de deslocamentos, intersecções e uniões usando a melhor solução;

NBS: número de EE's na melhor solução;

$\mathrm{T}$ : tempo para o algoritmo encontrar todas representações seqüenciais.

A Coluna 2 da Tabela 9.1 mostra que o número de intervalos (ou equivalentemente, o número de EE's) na sup-decomposição do operador apresentado na Coluna 1 . Na Coluna 3, mostramos o número de deslocamentos, intersecções e uniões efetuados pelo operador se implementamos na forma de sua sup-decomposição. A Coluna 4 apresenta 


$$
\begin{aligned}
& W_{1}=\begin{array}{l}
11111 \\
11111 \\
11111 \\
1111
\end{array}
\end{aligned}
$$

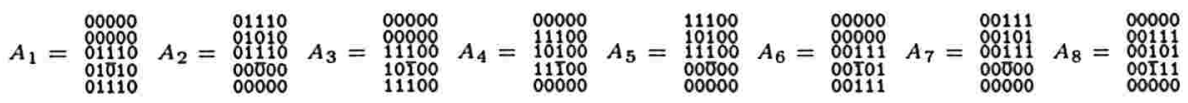

$$
\begin{aligned}
& \text { (b) }
\end{aligned}
$$

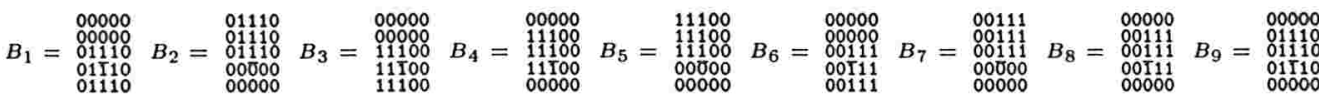

$$
\begin{aligned}
& \text { (c) }
\end{aligned}
$$

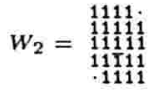

$$
\begin{aligned}
& \text { (d) }
\end{aligned}
$$

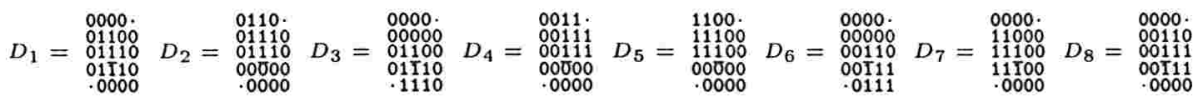

$$
\begin{aligned}
& \text { (e) } \\
& W_{3}=\underset{\substack{111111 \\
1.111}}{11111} \\
& \text { (f) }
\end{aligned}
$$

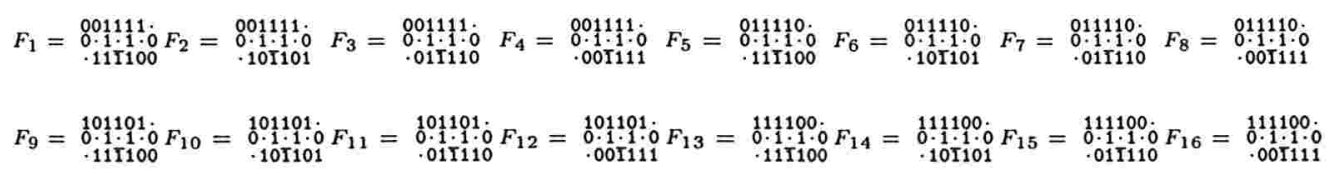

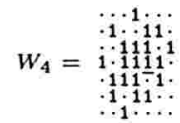

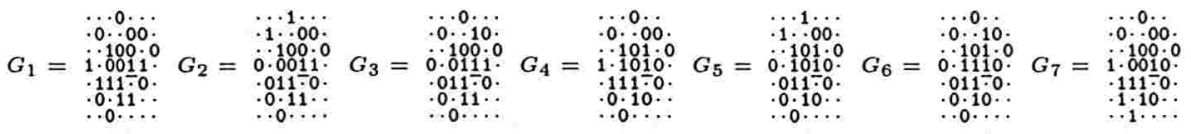

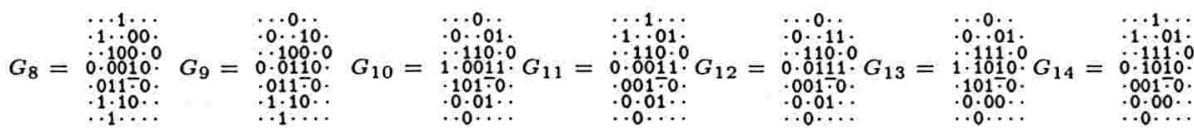

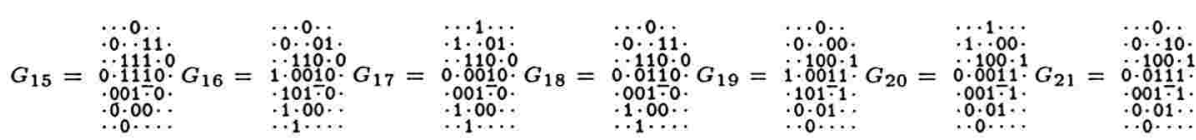

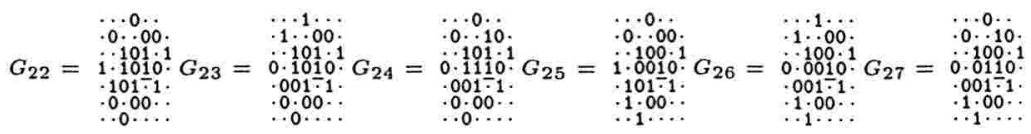




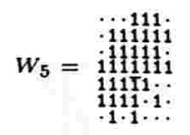

(j)

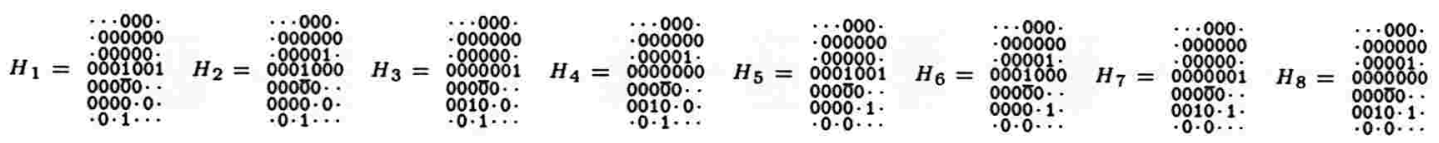

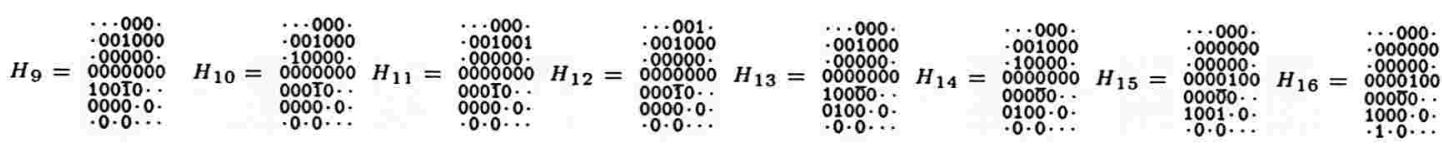

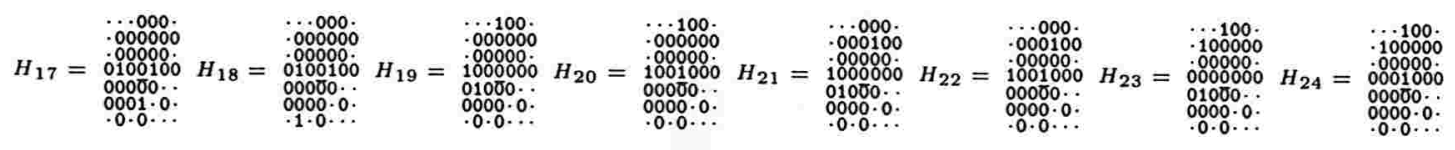

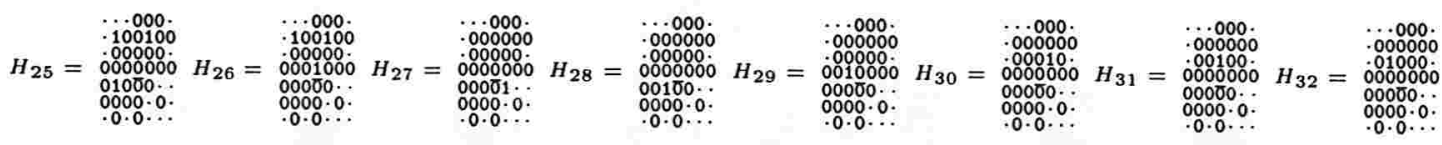

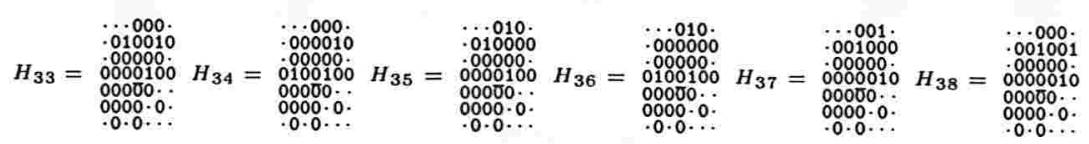

(k)

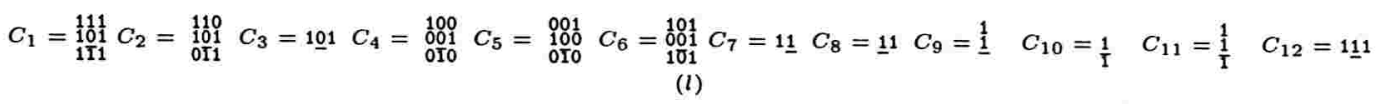

Figura 9.7: Alguns exemplos de transformação da sup-decomposição para decomposições seqüenciais.

o número de representações seqüenciais encontradas pelo algoritmo. A melhor solução encontrada pelo algoritmo é uma solução que usa o menor número de operações (isto é, deslocamentos, intersecções e uniões). Na Coluna 5, apresentamos o número de operações da melhor solução. A Coluna 6 mostra o número de EE's na melhor solução. O tempo gasto pelo algoritmo para encontrar todas representações seqüenciais estão apresentadas na Coluna 7 da Tabela 9.1. Comparando as Colunas 3 e 5, observamos que o número de operações na representação seqüencial é muito menor que na sup-decomposição.

Embora os resultados apresentados na Tabela 9.1 sejam bons, devemos observar que o algoritmo para encontrar a melhor representação seqüencial tem complexidade exponencial.

\subsection{Conclusão}

Neste capítulo, estudamos o problema de transformar a sup-decomposição de $W$-operadores em decomposições seqüenciais mais eficientes (quando elas existem). 


\begin{tabular}{ccccccc}
\hline Operador & NSD & OSD & NS & OBS & NBS & T \\
$\psi_{1}$ & 8 & 111 & 1 & 30 & 2 & $1.8 \mathrm{~s}$ \\
$\psi_{2}$ & 8 & 111 & 169 & 16 & 6 & $12.0 \mathrm{~s}$ \\
$\psi_{3}$ & 16 & 319 & 1 & 17 & 3 & $1.0 \mathrm{~s}$ \\
$\psi_{4}$ & 27 & 431 & 1 & 20 & 3 & $4.2 \mathrm{~s}$ \\
$\psi_{5}$ & 38 & 187 & 1 & 15 & 3 & $48 \mathrm{~m} 23 \mathrm{~s}$ \\
$\psi_{6}$ & 9 & 143 & 676 & 16 & 8 & $30 \mathrm{~m} 43 \mathrm{~s}$ \\
\hline
\end{tabular}

Tabela 9.1: Tempo utilizado pelo algoritmo para encontrar todas representações seqüenciais.

Definimos o problema da equação de fatoração de Minkowski e fornecemos limites superiores e inferiores para o espaço de soluções para o caso geral e para o caso de intervalos com extremidades direita fixa.

A teoria apresentada aqui foi implementada e aplicada para construir representações seqüenciais de operadores crescentes para os quais existem decomposições seqüenciais em termos de composições de dilatações e erosões.

Uma versão especializada deste problema é o problema clássico de encontrar decomposições seqüenciais para erosões e dilatações. De fato, um próximo passo para este trabalho pode ser encontrar somente os conjuntos viáveis $C$ de maior cardinalidade e posteriormente utilizar os resultados dos Capítulos 4, 5, 6 e 7 para decompor seqüencialmente $\delta_{C}$ ou $\varepsilon_{C^{t}}$.

Resumindo, estudamos formalmente o problema de transformar a sup-decomposição em representações seqüenciais e exploramos o problema estabelendo limites inferiores e superiores para o espaço de busca para solucioná-lo. No entanto, estamos longe de dar respostas definitivas para este problema tão complexo. O caminho aparenta ser bastante promissor, mas cheio de aventuras e desafios. 


\section{Capítulo 10}

\section{Conclusão Final}

Neste capítulo, na Seção 10.1, resumiremos e discutiremos os principais resultados desta tese. Na Seção 10.2, proporemos novos horizontes para a continuidade das pesquisas realizadas nesta tese.

\subsection{Conclusões e Contribuições}

Nesta seção, resumiremos as principais contribuições desta tese. Com estes resultados, foram acrescentados alguns avanços no estado da arte em MM, no que se refere à transformação de estruturas de decomposição.

O problema geral de mudança da sup-decomposição de um $W$-operador $\psi$ para outras estruturas de representação de $\psi$ que usam os operadores elementares de forma que o número de operações na MaqM seja mínimo é extremamente difícil e foi pouco explorado até o momento. Na literatura não há muitas referências a respeito deste problema. Apresentamos, neste trabalho, nos Capítulos 8 e 9, alguns resultados que vão na direção melhorar esta perspectiva, abrindo novos caminhos para pesquisas futuras nesta área ainda pouco explorada.

Como o problema geral de se encontrar a melhor representação de um operador, a partir de sua sup-decomposição, é extremamente difícil, muitos pesquisadores restrigiram o estudo desse problema para certas classes de operadores. De fato, existe um número considerável de estudos desse problema para a família das erosões e dilatações. No entanto, os estudos clássicos desse problema, restrito a famílias das erosões e dilatações, fornecem como resultados complexos algoritmos, onde o processo de otimização de uma solução encontrada está subdividido em vários intrincados subcasos. Além disso, as soluções exibidas por estes algoritmos podem ser melhoradas, ou seja, existem decomposições que implementam uma erosão ou uma dilatação de uma forma mais eficiente do que as decomposições geradas por esses algoritmos. Apresentamos, nesta tese, nos Capítulos 4, 5, 6 e 7, alguns resultados que melhoram estes estudos clássicos, introduzindo novos algoritmos 
mais simples e que geram soluções mais eficientes.

A seguir, nas Subseções de 10.1.1 a 10.1.5, detalhamos esses resutados comentados acima.

\subsubsection{Decomposição de Elementos Estruturantes Convexos}

Para o caso de EE's convexos, avançamos no estado da arte em MM apresentando um novo algoritmo que usa estratégia gulosa para decompor tais EE's. Os EE's que estão na decomposição gerada pelo algoritmo proposto não são necessariamente convexos. A complexidade desse algoritmo é $O\left(n^{3}\right)$, onde $n$ é o valor máximo entre as coordenadas do vetor retangular do EE convexo de entrada.

Embora a complexidade de tempo do algoritmo do Xu [49] seja $O\left(n^{2}\right)$, todos elementos na decomposição encontrada por este algoritmo são convexos.

Logo, como a complexidade computacional dos algoritmos que implementam erosões e dilatações depende do número de pontos do $\mathrm{EE}$, o algoritmo proposto tem uma vantagem sobre o algoritmo do Xu.

Uma outra qualidade de algoritmo proposto é que seu processo de otimização é muito simples comparado ao algoritmo do Xu.

Finalmente, uma análise teórica (prova de corretude e da complexidade de tempo) do algoritmo proposto foi apresentada.

\subsubsection{Decomposição de Elementos Estruturantes Simplesmen- te Conexos}

Para o caso de EE's simplesmente conexos, mostramos que o algoritmo de Park e Chin [35] para decompor tais EE's não decompõe três famílias infinitas de EE's decomponíveis simplesmente conexos. Isto significa que o algoritmo de Park e Chin é limitado no sentido de que ele não é capaz de decompor os EE's dessas famílias.

Teoricamente, o algoritmo de Park e Chin é um algoritmo polinomial, mas a sua implementação não é viável, uma vez que, em um determinado passo do algoritmo deles, é necessário encontrar uma solução inteira de um sistema linear com um número fixo de variáveis. De fato, para cada número inteiro positivo $n$, existe um algoritmo polinomial para encontrar soluções inteiras de sistemas de inequações lineares com $n$ variáveis [40, p. 256]. No entanto, este resultado é somente teórico, e a implementação desse algoritmo na prática não é viável. No caso geral, resolver sistemas lineares de solução inteira é um problema bastante difícil [40, p. 227]. 


\subsubsection{Decomposição de Elementos Estruturantes Arbitrários}

No caso geral, apresentamos um algoritmo que usa um método de "branch and bound", com estratégias de podas baseadas em propriedades geométricas e algébricas, deduzidas formalmente, da soma de Minkowski, para decompor EE's arbitrários. Esse algoritmo pode ser usado como prova automática de que uma erosão (respectivamente, dilatação) tem uma decomposição seqüencial ou não. A prova da existência é construtiva e uma solução ótima é exibida.

O algoritmo proposto não é eficiente para todos os casos, mas generaliza importantes resultados clássicos (Zhuang and Haralick, Xu, Park e Chin). Uma análise teórica do algoritmo, juntamente com alguns resultados experimentais, ilustra estes fatos. Em particular, mostramos que, se o EE de entrada $A$ é convexo, então o algoritmo encontra uma decomposição ótima de $A$ em tempo $O\left(n^{4}\right)$, onde $n=\max \left\{\rho_{0}(A), \rho_{1}(A)\right\}$.

O algoritmo combinatório apresentado pode ser melhorado se novos limites ou estratégias de podas forem descobertos.

\subsubsection{Representação Compacta de $W$-Operadores}

Introduzimos uma nova sup-representação e inf-representação para $W$-operadores: a representação compacta. A família de sup-geradores (respectivamente, inf-geradores) nesta representação é constituída de composições de sup-geradores da sup-decomposição com dilatações (respectivamente, de composições de inf-geradores da inf-decomposição com erosões).

Uma propriedade importante da representação compacta é que ela pode usar um número menor (ou, no pior caso, o mesmo número) de operadores sup-geradores (respectivamente, inf-geradores) que na sup-decomposição (respectivamente, inf-decomposição). Esta propriedade implica que os operadores são mais eficientes quando implementados na representação compacta.

Apresentamos também uma simplificação da representação compacta para a família de operadores idempotentes em um sentido estrito e anti-extensivos (extensivos). Além disso, a partir desta forma simplificada, obtemos uma realização minimal da representação de Matheron para aberturas e fechamentos IT's. Mostramos também que a sup-decomposição pode ser facilmente transformada para a sup-representação compacta e apresentamos alguns exemplos dessa transformação.

\subsubsection{Decomposição Seqüencial de $W$-Operadores}

Estudamos o problema de transformar a sup-decomposição de $W$-operadores em decomposições seqüenciais mais eficientes (quando elas existem). 
Definimos o problema da equação de fatoração de Minkowski e fornecemos limites superiores e inferiores para o espaço de soluções para o caso geral e para o caso de intervalos com extremidades direita fixa.

A teoria apresentada foi implementada e aplicada para construir representações seqüenciais de operadores crescentes para os quais existem decomposições seqüenciais em termos de composições de dilatações e erosões.

\subsection{Perspectivas Futuras}

Podemos comparar o trabalho de pesquisa com um trilhar por caminhos onde há trechos conhecidos e desconhecidos. O percurso conhecido já foi percorrido e marcado. O trecho desconhecido é vibrante e desafiador.

O percurso do caminho sobre problema geral de mudança da sup-decomposição de um $W$-operador $\psi$ para outras estruturas de representação de $\psi$ que usam os operadores elementares de forma que o número de operações na MaqM seja mínimo está apenas no início. Com os resultados desta tese, avançamos mais um pequeno, mas considerável, trecho desse caminho. No entanto, ainda falta percorrer um longo caminho para dar respostas definitivas para este problema. O caminho ainda desconhecido aparenta ser bastante promissor, mas cheio de aventuras e desafios.

Apresentamos, a seguir, alguns desafios que podem ser feitos a partir dos resultados desta tese.

O algoritmo DecCon apresentado no Capítulo 5 pode ser simplificado colocandose na seqüência SeqQ somente os elementos de SeqQ que são subconjuntos convexos do quadrado elementar. Com esta simplificação, poderíamos obter um algoritmo de mesma complexidade do algoritmo do $\mathrm{Xu}$.

A teoria apresentada no Capítulo 9 foi implementada e aplicada para construir representações seqüenciais de operadores que podem ser construídos por composições de dilatações e erosões.

Um próximo passo para este trabalho pode ser encontrar somente os conjuntos viáveis $C$ de maior cardinalidade e posteriormente utilizar os resultados dos Capítulos 4, 5, 6 e 7 para decompor seqüencialmente $\delta_{C}$ ou $\varepsilon_{C^{t}}$.

Outro próximo passo pode ser especializar este estudo para o caso de filtros alternados seqüenciais e tentar, usando algumas propriedades algébricas dessa classe de operadores, diminuir o espaço de busca para a solução da equação de fatorização de Minkowski.

Finalmente, podemos tentar encontrar algoritmos paralelos para os problemas que foram estudados nesta tese. 


\section{Apêndice A}

\section{Classificação de Texturas a partir de Transformações Morfológicas}

Uma ferramenta morfológica para classificação automática de texturas é proposta e aplicada a um enorme conjunto de amostras de revestimento de paredes. Começando a partir de medidas de dados morfológicos (covariança, velocidade de erosão e dilatação, distribuição de tamanho, densidade de picos e vales) sobre imagens em níveis de cinza, uma análise de correspondência é usada para aprender um procedimento de visualização de superfícies em um espaço reduzido, e, assim, selecionar as medidas mais pertinentes com respeito à classificação de texturas.

\section{A.1 Introdução}

A classificação de textura por análise de imagens tem muitas aplicações na indústria. O principal objetivo deste estudo é fornecer e demonstrar a utilidade de um algoritmo de classificação de textura que usa algumas medidas morfológicas e análise de dados estatísticos (análise de correspondência) para classificar texturas aleatórias em $k$ diferentes classes. Isto é aplicado para o desenvolvimento de um algoritmo rápido para classificar automaticamente algumas amostras de revestimento de paredes, para, posteriormente, usá-lo como um procedimento de inspeção visual em larga escala.

Este trabalho está dividido em dois passos: treinamento e classificação. No passo do treinamento, as medidas morfológicas feitas sobre imagens das amostras produzem uma enorme quantidade de dados, que são submetidos a uma análise estatítica para conseguir a melhor separação de representação de pontos em $k$ regiões. O passo de classificação é obtido pela projeção experimental de dados em uma dimensão menor que representa um sub-espaço dos dados medidos.

A principal dificuldade é encontrar as melhores medidas morfológicas que devem ser executadas. Neste trabalho, mostraremos que as medidas morfológicas: (i) curva de 


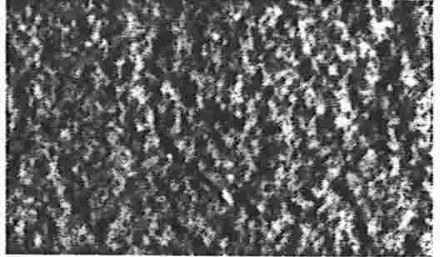

(a)

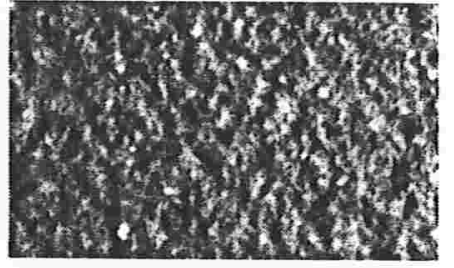

(b)

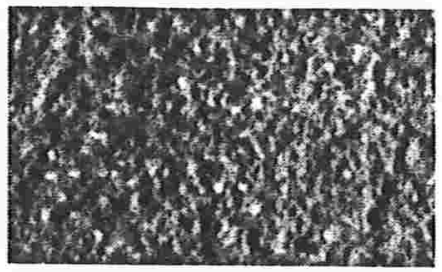

(c)

Figura A.1: (a) Qualidade $Q 1$ ("too early"), (b) Qualidade $Q 2$ ("normal"), (c) Qualidade $Q 3$ ("too late").

covariança; (ii) curvas de dilatação e erosão; e (iii) distribuição granulométrica e antigranulométrica não fornecem a mesma precisão para a classificação. Mostraremos como extrair as melhores funções morfológicas e os valores mais discriminantes para uma boa classificação das amostras. Este apêndice está organizado da seguinte forma. A Seção A.2 descreve as amostras estudadas. A Seção A.3 apresenta as medidas morfológicas utilizadas neste trabalho. A Seção A.4 descreve a análise estatística de dados. A Seção A.5 apresenta os resultados experimentais e finalmente, as conclusões estão resumidas na Seção A.6.

\section{A.2 Amostras e Aquisição de Imagens}

As amostras de revestimento de paredes estudadas neste trabalho são colocadas em um catálogo para serem dados aos clientes da indústria. As amostras produzidas apresentam três tipos de qualidades de texturas (denotadas por $Q 1, Q 2$ e Q3), devido a alguma instabilidade na elaboração destas texturas. Estas qualidades dependem do tempo de secagem do cimento antes de ser raspado. É possível definir as seguintes categorias, a partir do aspecto morfológico das amostras: "too early", "normal" e "too late". Alguns exemplos de tais amostras estão apresentados na Figura A.1

O problema de classificação estudado aqui é, dado uma coleção de imagens das amostras de um grupo $G$ contendo diferentes tipos de texturas, classificá-las em classes $Q 1, Q 2$ e $Q 3$, segundo o tipo de texturas.

Neste trabalho, apresentaremos um estudo com 300 imagens de amostras.

No processo de aquisição de imagens das amostras de revestimento de parede, um dispositivo simples composto por uma câmera e duas lâmpadas foram usadas. Uma iluminação normal e isotrópica incide obliquamente a 45 graus. As sombras, que o revelo da amostra projetam, fornecem uma textura não isotrópica. Nas imagens, os níveis de cinza não representam diretamente a elevação do ponto considerado, mas são, no entanto, representantes da topografia: as imagens obtidas apresentam sombras, devido a presença forte de relevos (Figura A.1).

Todas imagens adquiridas apresentaram um ruído periódico horizontal, que foi removido por um filtro de freqüência, usando a Transformação Rápida de Fourier (TRF). As 
imagens finais contêm $485 \times 285$ pixels.

\section{A.3 Medidas Morfológicas}

Quando se trabalha com texturas, é conveniente considar as imagens como realizações de estruturas aleatórias (conjuntos aleatórios no caso de imagens binárias [32, 41], e funções aleatórias para imagens em níveis de cinza). Estas estruturas aleatórias podem ser caracterizados pela capacidade de Choquet [32, 41, 25], a partir do qual são derivados a maioria das medidas morfológicas.

\section{A.3.1 A Capacidade de Choquet}

Uma função aleatória, semicontínua, limitada superiormente $Z(x)$ é caracterizada pela sua capacidade de Choquet, $T(g)$, que pode ser definida [25] sobre funções contínuas limitadas inferiormente $g$ com um suporte compacto $K$ :

$$
T(g)=P\left\{x \in D_{Z}(g)\right\} ; D_{Z}(g)^{c}=\{x, Z(y)<g(y-x), \forall y \in K\}
$$

Um caso particular e muito utilizado é a lei espacial definida sobre um número finito de pontos $x_{1}, x_{2}, \ldots, x_{n}$ da seguinte forma,

$$
F\left(x_{1}, x_{2}, \ldots, x_{n}, z_{1}, z_{2}, \ldots, z_{n}\right)=P\left\{Z\left(x_{1}\right)<z_{1}, Z\left(x_{2}\right)<z_{2}, \ldots, Z\left(x_{n}\right)<z_{n}\right\}
$$

Outros casos particulares fornecem as transformações por $\vee$ (supremo), chamado uma dilatação ou por $\wedge$ (ínfimo), chamado uma erosão, que geram novas funções aleatórias:

$$
\begin{aligned}
& Z_{\vee}(K)=\vee_{x \in K}\{Z(x)\}=Z \oplus K \\
& Z_{\wedge}(K)=\wedge_{x \in K}\{Z(x)\}=Z \ominus K
\end{aligned}
$$

Como um caso particular, quando o conjunto compacto $K$ é limitado no ponto $x$ e $g(x)=z$, recobrimos a função distribuição $F(z)$ :

$$
F(z)=P\{Z(x)<z\}=1-T(g)
$$




\section{A.3.2 Medidas Morfológicas}

\section{A.3.2.1 Função de Covariança}

A função de covariança centrada e reduzida de uma função aleatória estacionária $Z(x)$ é definida por:

$$
C(h)=\frac{E\{Z(x) Z(x+h)\}-(E\{Z(x)\})^{2}}{E\{Z(x) Z(x)\}-(E\{Z(x)\})^{2}}
$$

onde, $E\{\cdot\}$ denota a esperança matemática de uma variável aleatória. O termo $E\{Z(x)$ $Z(x+h)\}$ é estimado pela média do produto $\{Z(x) Z(x+h)\}$ sobre os pixels das imagens. Como este produto pode ser interpretado em termos de um produto de convolução, ele é obtido por TRF. Adicionamos o valor 0.5 para a covariança centrada e reduzida, dessa forma, evitando valores negativos para a análise de correspondência. As medidas foram feitas por valores $h$ entre 1 to 100 nas duas principais direções da imagem (horizontal e vertical).

\section{A.3.2.2 Curva de Abertura e Fechamento (Antigranulometria e Granulome- tria)}

Uma granulometria é o estudo de distribuições de tamanho dos objetos em uma imagem [32, 41]. Calculamos, ao mesmo tempo, uma granulometria (distribuições de tamanhos de picos) usando aberturas morfológicas e uma antigranulometria (distribuições de tamanhos de vales) usando fechamentos morfológicos. O seguinte estimador para a densidade de tamanhos é equivalente a uma função de densidade de probabilidade, enquanto a função $S(k)$ tem o papel de uma função distribuição acumulativa [23]:

$$
\tilde{S}=S(k)-S(k+1)
$$

com,

$$
S(k)=\frac{1}{N} \cdot \frac{1}{\operatorname{Vol}\{M \ominus 2 k B\}} \cdot \begin{cases}\operatorname{Vol}\{(M \ominus 2 k B) \cap((Z-\operatorname{Av}\{Z\}) \circ k B)\} & \text { if } k \geq 0 \\ \operatorname{Vol}\{(M \ominus 2|k| B) \cap((Z-\operatorname{Av}\{Z\}) \bullet|k| B)\} & \text { if } k<0\end{cases}
$$

onde:

- $Z \circ k B$ é a abertura de $Z$ por $k B$,

- $Z \bullet|k| B$ é o fechamento de $Z$ por $k B$,

- $N$ é o número de níveis de cinza na imagem (no nosso caso $N=255$ ),

- Vol $\{\cdot\}$ é o volume da imagem, ou seja, $\operatorname{Vol}\{X\}=\sum_{w \in X} X(w)$, 
- $\operatorname{Av}\{\cdot\}$ é a imagem média constante, ou seja, $\operatorname{Av}\{X\}$ é uma imagem de mesmo tamanho que $X$ com altura constante igual a $\frac{1}{S} \cdot \operatorname{Vol}\{X\}$, onde $S$ é o número de pixels de $X$,

- $Z$ é a imagem de entrada,

- $M$ é a imagem máscara,

- $B$ é o elemento estruturante (quadrado; segmento horizontal ou vertical), e

- $M \cap Z$ é a restrição da imagem $Z$ à mascara $M$.

A Figura A.2 fornece um exemplo de curvas de abertura e fechamento. O lado direito (valores positivos do eixo horizontal) é a curva de abertura enquanto que o lado esquerdo (valores negativos do eixo horizontal) é a curva de fechamento.

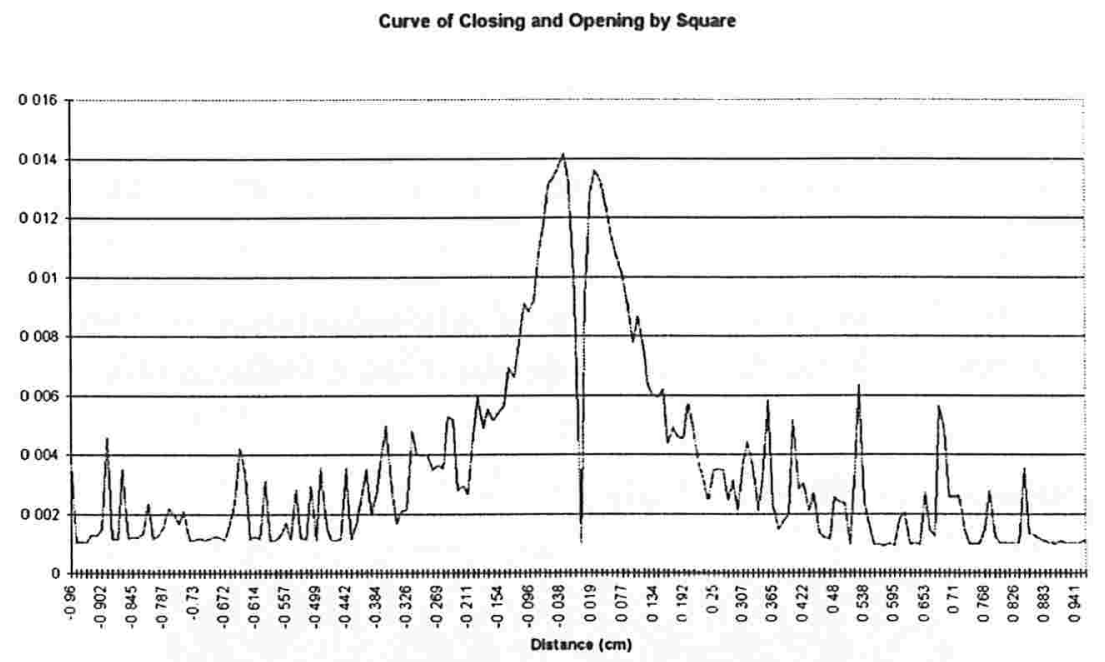

Figura A.2: Exemplo de uma curva de fechamento (lado esquerdo) e uma curva de abertura (lado direito) por elementos estruturantes quadrados.

\section{A.3.2.3 Curva de Erosão e Dilatação}

Nós também usamos uma "pseudo-granulometria" e "pseudo-antigranulometria" obtidas por uma substituição do fechamento e abertura por, respectivamente, dilatação e erosão [23]. Mais formalmente,

$$
S(k)=\frac{1}{N} \cdot \frac{1}{\operatorname{Vol}\{M \ominus k B\}} \cdot \begin{cases}\operatorname{Vol}\{(M \ominus k B) \cap((Z-\operatorname{Av}\{Z\}) \ominus k B)\} & \text { if } k \geq 0 \\ \operatorname{Vol}\{(M \ominus|k| B) \cap((Z-\operatorname{Av}\{Z\}) \oplus k B)\} & \text { if } k<0\end{cases}
$$

A velocidade da erosão é definida como a diferença $|S(k)-S(k+1)|$. Na Figura A.3 fornece um exemplo de uma curva de erosão e dilatação por segmentos verticais. Como 
no caso da granulometria, erosões e dilatações por quadrados (com tamanhos entre 1 a 100 pixels), segmentos horizontais e verticais (com tamanhos entre 1 a 100 pixels) foram usadas para a classificação de texturas.

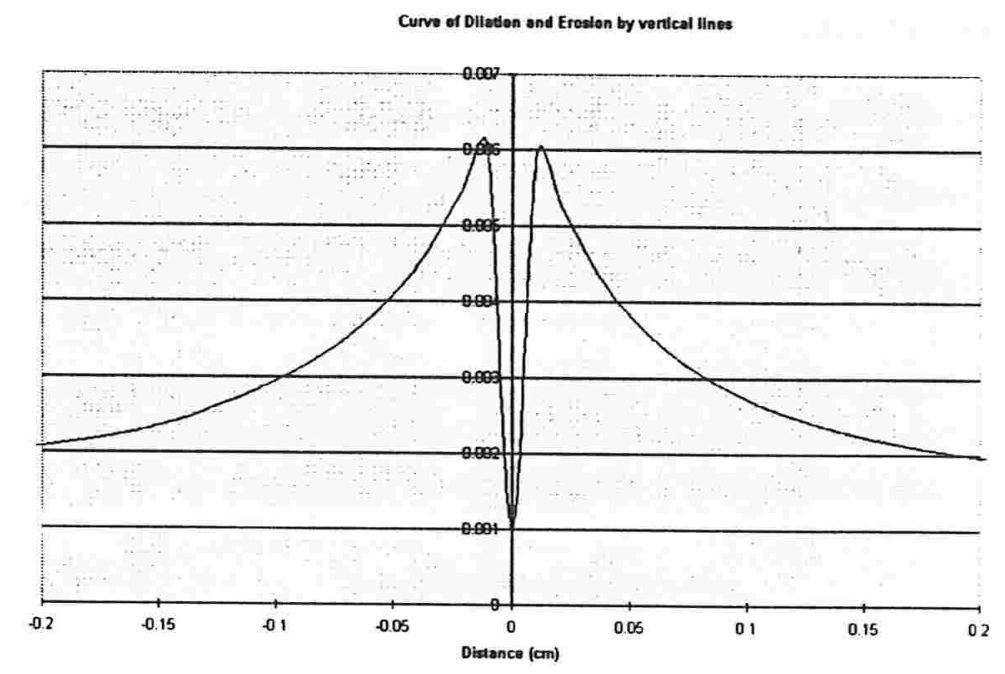

Figura A.3: Exemplo de uma curva de erosão (lado direito) e uma curva de dilatação (lado esquerdo) por segmentos verticais.

Observamos o cálculo das curvas de erosão e dilatação pode ser feito de uma maneira muito mais rápida que o cálculo das curvas de abertura e fechamento.

\section{A.3.2.4 Medidas sobre Picos e Vales}

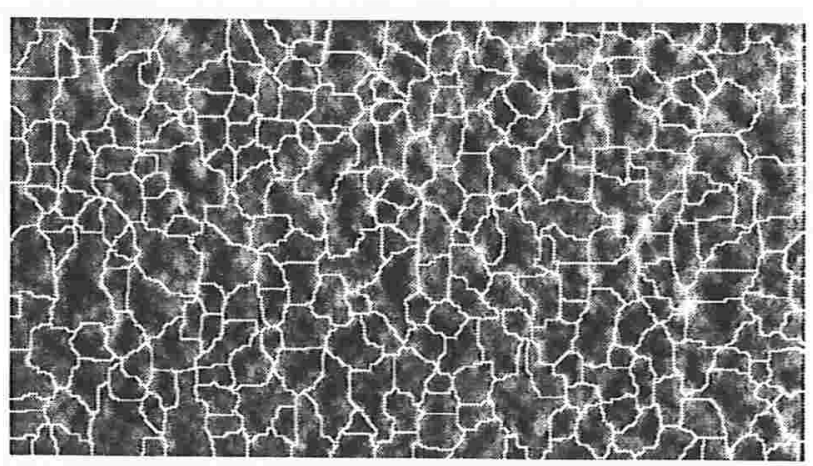

Figura A.4: Exemplo de "watersheds"

Para se obter informação sobre a conectividade dos picos e vales, extraímos os "watersheds" das imagens (um exemplo é dado na Figura A.4). A partir dessas imagens segmentadas, medimos o número de vales com uma dada área $S$, ou com um dado volume $V$ (pela integração dos valores dos níveis de cinza para cada "watershed") e, analogamente, para o número de picos. Este tipo de informação está ilustrado na Figura A.5, onde 
a parte esquerda refere-se aos picos e a parte direita aos vales. Estes dados morfológicos foram usados em um estudo preliminar de superfícies, mas não foram relevantes para a classificação das texturas deste trabalho.

Curve of Area Histogram

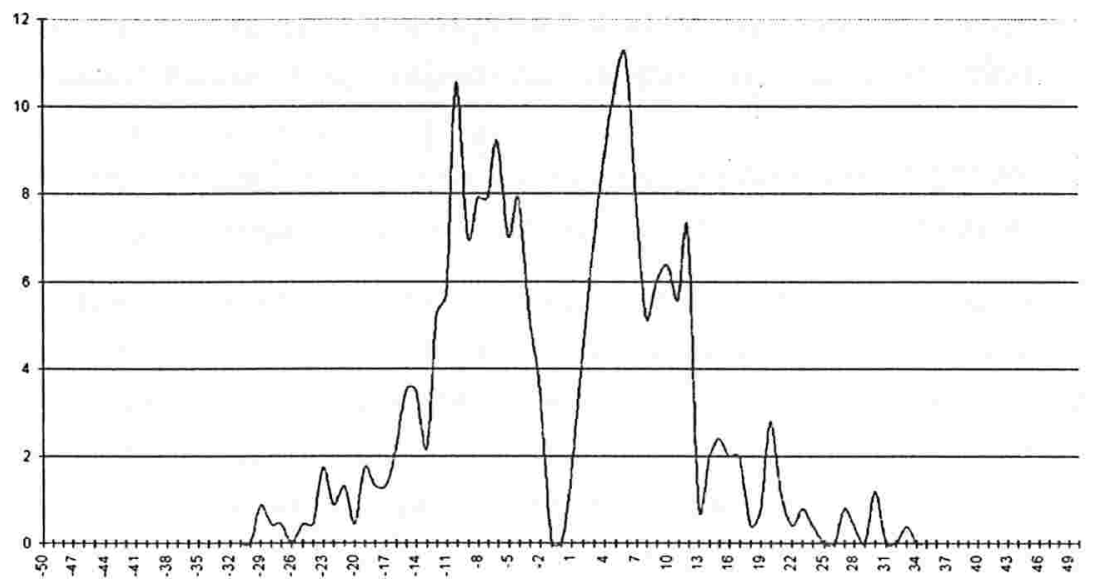

Figura A.5: Exemplo da distribuição de picos (parte esquerda) e de vales (parte direita)

\section{A.4 Introdução à Análise de Correspondências}

Para analisar as medidas morfológicas descritas na Seção A.3.2 e produzir uma classificação das amostras, usamos a análise de correspondência [13]. Um tratamento análogo foi feito em [26] para a classificação automática de inclusões não metálicas em rodas. Foi usado mais recentemente, para a classificação de superfícies rugosas [2], e para simulações de conjuntos aleatórios [3]. Os valores das medidas estão armazenados em uma tabela, onde toda linha corresponde a uma amostra (ou a uma observação) e toda coluna corresponde a uma medida (ou a uma variável). Definimos a coordenada linha de uma dada superfície (observação) como o vetor de $M$ dimensões, e a coordenada coluna de uma dada medida (variável) como um vetor de $N$ dimensões.

Técnicas alternativas de análise de fatores poderiam ser utilizadas para este propósito, como análise de fatores discriminantes, ou ainda análise de discriminantes penalizados [46]. Estas análises dividem com a análise de correspondência o fato de que uma combinação linear ótima das variáveis é procurada para a discriminação dos dados, e, na prática, estas análises fornecem resultados muito similares. Além disso, pode-se provar que a análise de correspondência é um caso especial da análise de fatores discriminantes, baseado sobre a métrica do "chi" quadrado [13].

A análise de correspondência permite construir um mapeamento das coordenadas linhas e colunas (pontos com $M$ e $N$ dimensões, respectivamente) em um espaço de di- 
mensão reduzida (por exemplo, um espaço de dimensão dois) gerando critérios sintéticos (neste sentido, o método é um tipo de análise de fator [13]).

Nem todas as variáveis utilizadas na análise são importantes para separar as amostras. Esta análise permite encontrar as variáveis mais significativas entre as variáveis iniciais para a separação das amostras.

A análise de correspondência permite uma representação de nuvens de pontos linhas e colunas em um espaço reduzido, que separa na medida do possível todos pontos por uma maximização da inércia. Além disso, esta análise permite a interpretação dos eixos (ou fatores) do plano da representação em termos das variáveis iniciais. Isto é precisamente a principal vantagem deste método (com respeito à análise clássica de fatores).

Com uma indicação da qualidade dos eixos sintéticos é possível estimar a precisão da representação simplificada e a perda de informação, a partir da parte correspondente à inércia que cada eixo carrega. Por exemplo, se a primeira direção significa $45 \%$ de inércia, então ela sintetiza $45 \%$ da informação total. Obviamente, uma representação de pontos em um plano significa a soma de frações de inércia para cada eixo. Isto é importante para observar que podemos usar a "distância" entre imagens, bem como a "distância" entre as variáveis.

No exemplo do estudo mostrado na Figura A.6, observamos que a parte de inércia é $88.4 \%$ para o primeiro fator e $5.8 \%$ para o segundo. Isto significa que um vetor de 200 dimensões pode ser representado em um espaço de 2 dimensões mantendo o total de $95 \%$ de informação. A visualização dos pontos colunas permite identificar as medidas mais discriminantes. Para concluir esta parte, lembramos que a análise de correspondência permite:

- Visualizar as superfícies em um espaço reduzido (tipicamente 2 ou 3 dimensões) com a ajuda de fatores sintéticos.

- Dar uma interpretação para esta representação.

- Propor a redução do número de variáveis, útil para diminuir o tempo de medida para a etapa de classificação.

\section{A.5 Resultados Experimentais}

O estudo deste trabalho foi feito em três etapas. Na primeira, a importância dos elementos estruturantes direcionais são estimados (esta escolha é justificada pela iluminação direcional feita na aquisição das imagens das amostras). Em uma segunda etapa, as variáveis mais discriminantes são determinadas, a fim de reduzir o número de dados. Estas duas primeiras etapas foram feitas em uma base de dados reduzido (30 imagens de amostras). $\mathrm{Na}$ etapa final, estudamos a base de dados completa (300 imagens de amostras) sobre um número reduzido de medidas. 


\section{A.5.1 Primeira Etapa: Análise das Medidas}

A primeira parte do estudo pretende avaliar se as texturas obtidas são realmente determinantes para caracterizar as diferentes qualidades do produto. As curvas de abertura e fechamento não forneceram bons resultados discriminantes, principalmente devido a fato que as curvas experimentais apresentaram muitas oscilações (veja a Figura A.2). Imagens maiores de cada amostra seriam requisitadas para proceder a análise estatística. Na parte restante do estudo, limitamos as medidas para as curvas de erosão e dilatação (e, assim, abandonando as medidas de abertura e fechamento). Elementos estruturantes direcionais aparentaram ser bons para a investigação desse estudo.

\section{A.5.2 Segunda Etapa: Redução do Número de Variáveis}

As projeções das variáveis no primeiro plano de fatores mostram a influência de cada variável sobre a discriminação. De fato, as variáveis mais dispersas nos planos de fatores são mantidas (duas variáveis próximas terão a mesma influência sobre a representação e classificação, e, portanto, uma só pode ser utilizada). Para a parte restante do estudo, é possível manter as seguintes medidas:

- A curva de covariança (horizontal e vertical), para distâncias entre 1 e 50 pixels.

- As curvas de erosão e dilatação, usando como elementos estruturantes segmentos horizontais e verticais com tamanho entre 1 e 30 pixels.

- As curvas de erosão e dilatação, usando elementos estruturantes quadrados com lados entre 1 e 50 pixels.

As medidas foram feitas para uma base de dados com 100 amostras de cada tipo de qualidade.

\section{A.5.3 Estudo Final}

Primeiramente, um resultado muito importante deste estudo é que a função de covariança não é suficientemente eficiente para discriminar as três categorias das amostras, enquanto que as operações morfológicas (erosão e dilatação) fornecem discriminação satisfatória das amostras. Usando uma simples regressão linear para definir os melhores limites para os diferentes domínios, obtemos, para estes exemplos, as pontuações dadas nas Figuras A.6 e A.7. Observamos que, no caso de operadores com elementos estruturantes com direção perpendicular à incidência da iluminação (Figura A.7), estes fornecem o melhor resultado (90\% de pontuação da classificação). Isto é muito interessante, pois existem algoritmos muito rápidos para calcular erosões e dilatações direcionais. Como mostrado nas Figuras A.6 e A.7, algumas amostras não foram classificadas corretamente. A razãos para 
a classificação que não foi feita corretamente é que as texturas das amostras são muito similares às correspondentes amostras das outras famílias. Devemos observar que não é fácil separar as amostras por uma simples inspeção manual.

\section{A.6 Conclusão}

Apresentamos uma ferramente automática de classificação de texturas. Esta ferramente, usada aqui, é um estudo prático e baseado na análise de correspondência de dados morfológicos. Esta análise permite a visualização em um espaço sintético (aqui de duas dimensões), onde o significado de cada eixo é obtido pela projeção das variáveis neste mesmo espaço. A partir de observações das variáveis neste plano, as variáveis mais discriminantes podem ser extraídas. A classificação automática baseada nas curvas de erosão e dilatação (usando elementos estruturantes quadrados ou segmentos horizontais e verticais) fornecem resultados muito satisfatórios que a função de covariança neste estudo. Isto é consistente com a caracterização teórica de estruturas aleatórias pela capacidade de Choquet. Trabalhando-se com poucos dados otimizados (curvas de erosão e dilatação com elementos estruturantes de tamanho 30 ou 50), podemos obter uma boa classificação de texturas muito similares, com a pontuação de $90 \%$. O controle de amostras mal classificadas mostra que elas são muito difícies de discriminar, mesmo para um observador experiente.

Este estudo é, de fato, em uma primera fase, uma aprendizagem para a classificação: usando a base de dados de referência, as melhores direções (ou fatores) que discriminam estas amostras são calculadas. Posteriormente, em uma segunda fase, para classificar uma nova amostra, temos que somente utilizar a projeção dela nestas direções calculadas no passo anterior (isto é, usa-se os resultados da etapa da aprendizagem, não é necessário recalcular novos fatores). Como resultado deste estudo, aparentemente erosões e dilatações lineares são bastante discriminantes para texturas muito similares, onde a covariança não funciona para separar tais amostras de superfícies.

\section{A.7 Agradecimentos}

Este estudo foi feito com a apoio financeiro da empresa "Weber \& Broutin", que forneceu as amostras de revestimento de parede. Este trabalho também teve o apoio financeiro do CNPq, proc. 200973/96-7. 
Curve Erosion and Dilation by Square

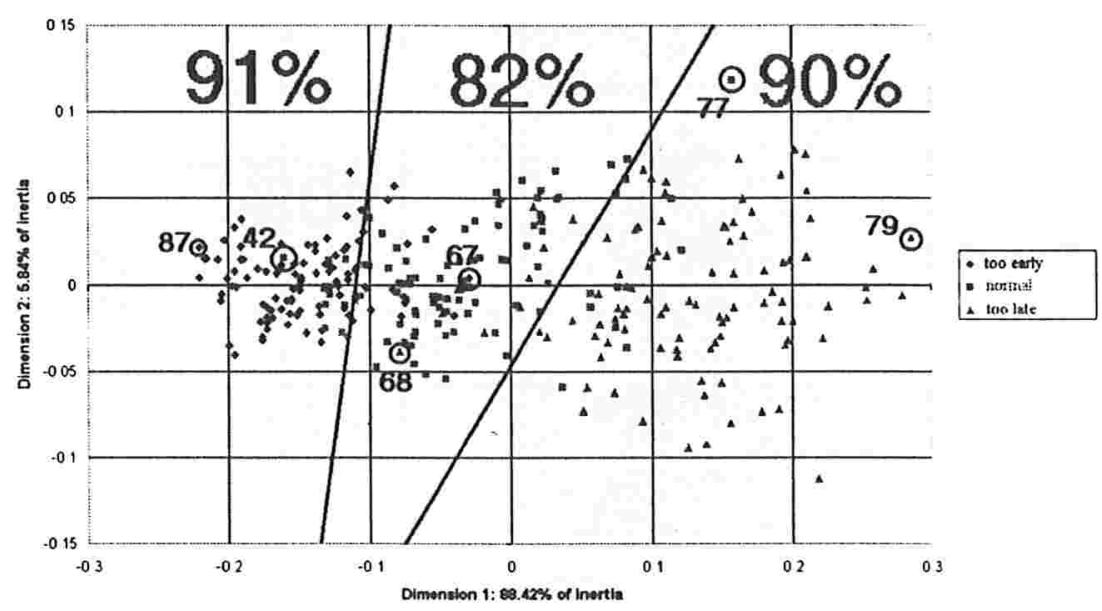

Curve Erosion and Dilation by Square Gratte 2

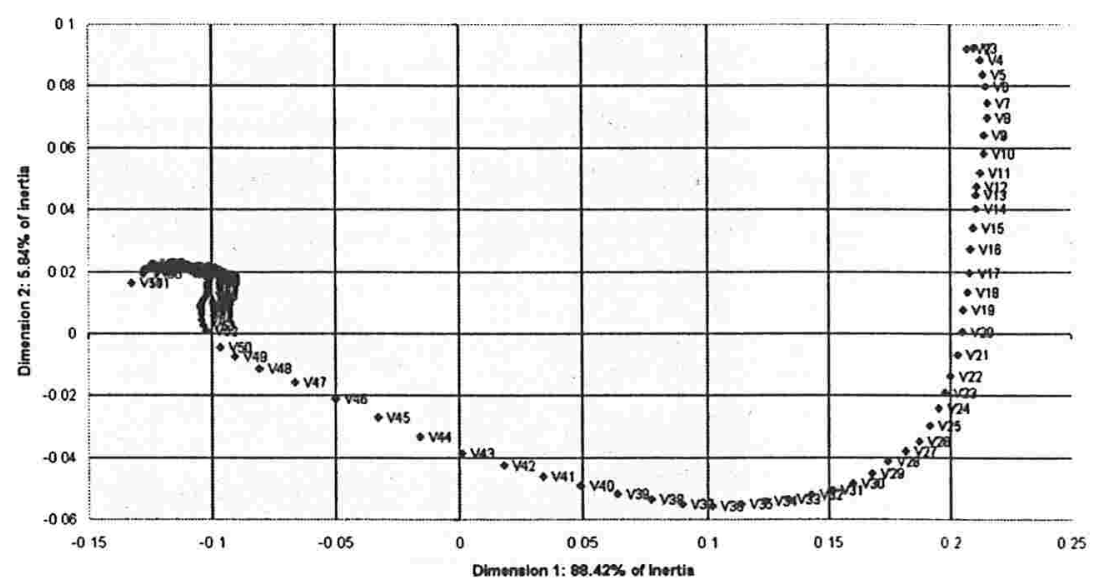

Figura A.6: Pontuação da classificação para as curvas de erosão e dilatação pelo quadrado e a correspondente representação das variáveis no primeiro plano de fatores. 

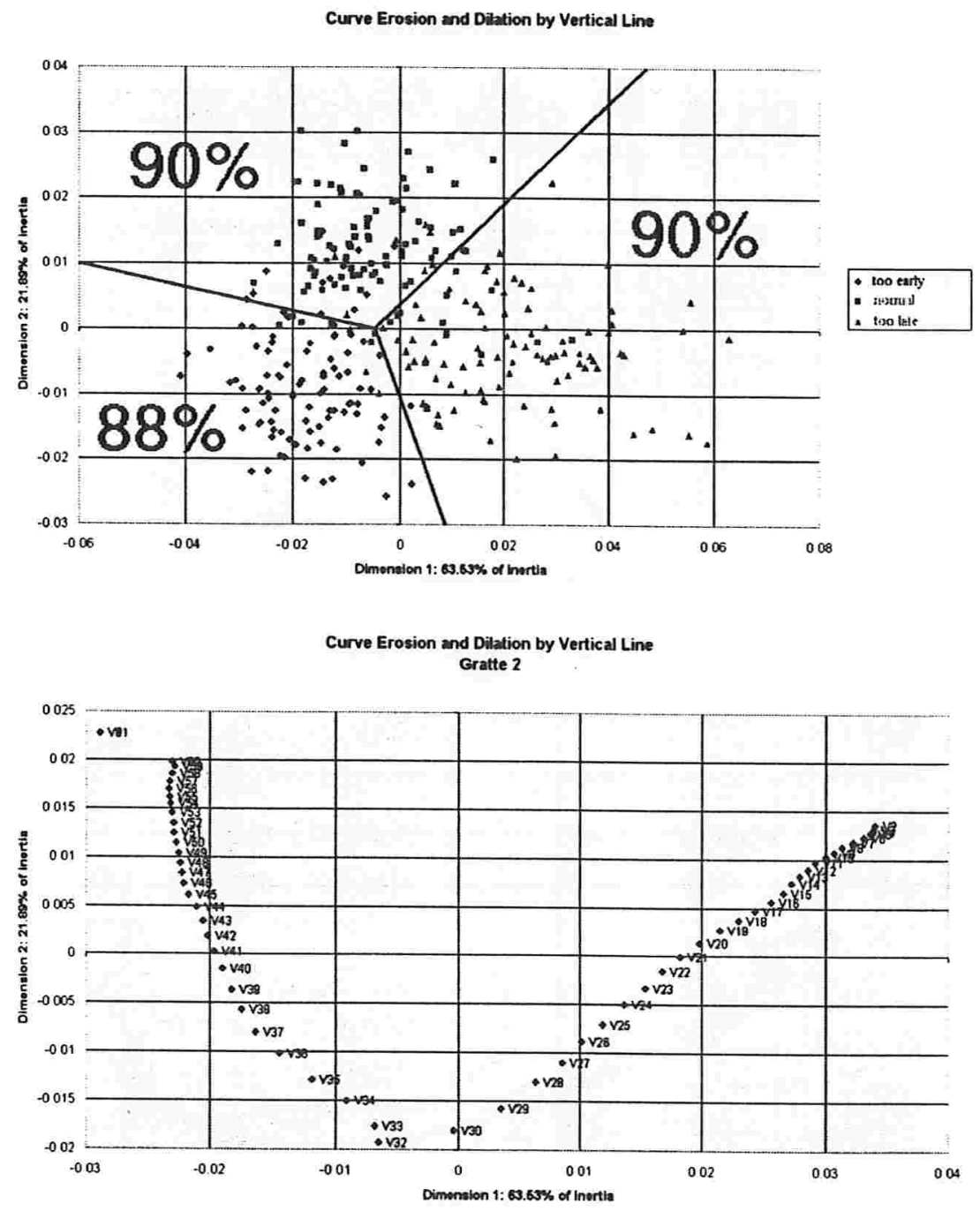

Figura A.7: Pontuação da classificação para as curvas de erosão e dilatação por linhas verticais e a correspondente representação das variáveis no primeiro plano de fatores. 


\section{Apêndice B}

\section{Artigos Publicados e Submetidos a Revistas e Congressos}

\section{Artigos Completos Publicados e Submetidos em Pe- riódicos}

1. R. F. Hashimoto, J. Barrera and C. E. Ferreira. A Combinatorial Optimization Technique for the Sequential Decomposition of Erosions and Dilations. Journal of Mathematical Imaging and Vision, 13(1):17-33, August, 2000.

2. R. F. Hashimoto and J. Barrera. A Greedy Algorithm for Decomposing Convex Structuring Elements. IEEE Transactions on Pattern Analysis and Machine Intelligence, 2000. Paper Submitted.

3. R. F. Hashimoto and J. Barrera. A Note on Park and Chin's Algorithm. IEEE Transactions on Pattern Analysis and Machine Intelligence, 2000. Paper Submitted.

4. R. F. Hashimoto and J. Barrera. From the Sup-Decomposition to Sequential Decompositions. Journal of Mathematical Imaging and Vision, 2000. Paper Submitted. 


\section{Trabalhos Completos Publicados em Eventos}

1. R. F. Hashimoto, J. Barrera and E. R. Dougherty. From the Sup-Decomposition to a Sequential Decomposition. In International Symposium On Mathematical Morphology (ISMM'2000), Palo Alto. Mathematical Morphology and its Applications to Image and Signal Processing. Kluwer Academic Publishers, 2000. p.13-22.

2. A. Aubert, D. Jeulin and R. F. Hashimoto. Surface Texture Classification from Morphological Transformations. In International Symposium On Mathematical Morphology (ISMM'2000), Palo Alto. Mathematical Morphology and its Applications to Image and Signal Processing. Kluwer Academic Publishers, 2000. p. 253-262.

3. R. F. Hashimoto and J. Barrera. A Simple Algorithm for Decomposing Convex Structuring Elements. In XII Brazilian Symposium on Computer Graphics and Image Processing, 1999, Campinas. Proceedings of SIBGRAPI'99. Los Alamitos: IEEE Computer Society, 1999. p.275-282.

4. R. F. Hashimoto. An Extension of an Algorithm for Finding Sequential Decomposition of Erosions and Dilations. In International Symposium on Computer Graphics, Image Processing and Vision, 1998, Rio de Janeiro. Proceedings SIBGRAPI'98. Los Alamitos: IEEE Computer Society, 1998. p.443-449.

5. J. Barrera and R. F. Hashimoto. Compact Representation of W-Operators. In 10th Annual Symposium on Eletronic Imaging, 1998, San Jose. SPIE Press, 1998. p.84-94.

6. J. Barrera, C. E. Ferreira and R. F. Hashimoto. Finding Optimal Sequential Decompositions of Erosions and Dilations. In International Symposium on Mathematical Morphology (ISMM'98), 1998, Amsterdam. Mathematical Morphology and its Applications to Image and Signal Processing. Kluwer Academic Publishers, 1998. v.12. p.299-306.

7. J. Barrera, G. P. Salas and R. F. Hashimoto. Set Operations on Closed Intervals and their applications to the Automatic Programming of MMACH's. In International Symposium on Mathematical Morphology, 1996, Atlanta. Mathematical Morphological and its Applications to Image and Signal Processing. Norwell: Kluwer Academic Publishers, 1996. v.11. p.377-384. 


\section{Referências Bibliográficas}

[1] G. Anelli, A. Broggi, and G. Destri. Decomposition of Arbitrarily Shaped Binary Morphological Structuring Elements Using Genetic Algorithms. IEEE Transactions on Pattern Analysis and Machine Intelligence, 20(2):217-224, February 1998.

[2] A. Aubert and D. Jeulin. Classification Morphologique de Surfaces Rugueuses. To appear in Revue de Métallurgie, February 2000.

[3] A. Aubert and D. Jeulin. Estimation of the Influence of Second and Third Order Moments on Random Sets Reconstructions. Pattern Recognition, 33(6):1083-1103, April 2000.

[4] S. Baase. Computer Algorithms. Addison-Wesley, 1988.

[5] D.M. Ballard and C.M. Brown. Computer Vision. Prentice Hall, Englewood Cliffs, New York, 1977.

[6] G. J. F. Banon and J. Barrera. Minimal Representations for Translation-Invariant Set Mappings by Mathematical Morphology. SIAM J. Appl. Math., 51(6):1782-1798, December 1991.

[7] G. J. F. Banon and J. Barrera. Decomposition of Mappings between Complete Lattices by Mathematical Morphology, Part I. General Lattices. Signal Processing, 30:299-327, 1993.

[8] G. J. F. Banon and J. Barrera. Bases da Morfologia Matemática para Análise de Imagens Binárias. IX Escola de Computação, Pernambuco, 1994.

[9] J. Barrera and G. J. F. Banon. Expressiveness of the Morphological Language. In Image Algebra and Morphological Image Processing III, volume 1769, pages 264-274, San Diego, California, 1992. SPIE.

[10] J. Barrera, F.S.C. da Silva, and G. J. F. Banon. Automatic Programming of Binary Morphological Machines. In Image Algebra and Morphological Image Processing, volume 2300, pages 229-240, San Diego, 1994. SPIE Proceedings. 
[11] J. Barrera, E. R. Dougherty, and N. S. Tomita. Automatic Programming of Binary Morphological Machines by Design of Statistically Optimal Operators in the Context of Computational Learning Theory. Journal of Electronic Imaging, 6(1):54-67, January 1997.

[12] J. Barrera and G. P. Salas. Set Operations on Collections of Closed Intervals and their Applications to the Automatic Programming of Morphological Machines. Journal of Eletronic Imaging, 5(3):335-352, July 1996.

[13] J. P. Benzécri. L'analyse des Données: L'analyse des Correspondances. Dunod, Paris, 1973.

[14] G. Birkhoff. Lattice Theory. American Mathematical Society, Providence, Rhode Island, 1967.

[15] T. H. Cormen, C. E. Leiserson, and R. L. Rivest. Introduction to Algorithms. McGraw-Hill, 1990.

[16] R. O. Duda and P. E. Hart. Pattern Classification and Scene Analysis. John Wiley and Sons, 1973.

[17] E. A. Filho. Teoria Elementar dos Conjuntos. Livraria Nobel S.A., São Paulo, 1980.

[18] H. Freeman. Computer Processing of Line-Drawing Images. Computer Surveys, 6(1):57-97, March 1974.

[19] P. Gader. Separable Decompositions and Approximations for Gray-Scale Morphological Templates. Computer Vision, Graphics, and Image Processing, 53:288-296, July 1991.

[20] P. Gader and S. Takriti. Decomposition Techniques for Gray-Scale Morphological Templates. volume 1350, pages 431-442. SPIE Press, July 1990.

[21] M. R. Garey and D. S. Johnson. Computers and Intractability - A Guide to the Theory of NP-Completeness. W. H. Freeman and Company, San Francisco, 1979.

[22] R. C. Gonzalez and R. E. Woods. Digital Image Processing. Addison-Wesley Publishing Company, 1992.

[23] J. Goutsias and K. Sivakumar. Discrete Morphological Size Distributions and Densities: Estimation Techniques and Applications. Journal of Electronic Imaging, 6:31-53, January 1997.

[24] H. J. A. M. Heijmans. Morphological Image Operators. Academic Press, 1994.

[25] D. Jeulin. Modèles Morphologiques de Structures Aléatoires et de Changement d'Échelle. PhD thesis, University of Caen, April 1991. 
[26] D. Jeulin and J. Serra. Pour Reconnaitre les Inclusions: Chartes ou Analyseurs de Textures? Mémoires et Etudes Scientifiques de la Revue de Métallurgie, 72:745-751, October 1975.

[27] R. Jones. The Transformation of the Basis Representation into Cascaded Representations. In Mathematical Morphology and its Applications to Signal Processing, pages 239-244, Barcelona, Spain, 1993.

[28] T. Kanungo and R. Haralick. Vector-Space Solution for a Morphological ShapeDecomposition Problem. Journal of Mathematical Imaging and Vision, 2:51-82, 1992.

[29] T. Y. Kong and A. Rosenfeld. Digital Topology: Introduction and Survey. Computer Vision, Graphics and Image Processing, 48:357-393, 1989.

[30] P. A. Maragos. A Unified Theory of Translation-Invariant Systems with Applications to Morphological Analysis and Coding of Images. PhD thesis, School of Elect. Eng. - Georgia Inst. Tech., 1985.

[31] David Marr. Vision. W. H. Freeman, 1982.

[32] G. Matheron. Random Sets and Integral Geometry. John Wiley, 1975.

[33] P. M. Pardalos P. Sussner and G. X. Ritter. On Integer Programming Approches for Morphological Template Decomposition Problems in Computer Vision. In D. Du, editor, In Journal of Combinatorial Optimization, volume 1, pages 165-178. Kluwer Academic Publishers, 1997.

[34] H. Park and R. T. Chin. Optimal Decomposition of Convex Morphological Structuring Elements for 4-Connected Parallel Processors. IEEE Transactions on Pattern Analysis and Machine Intelligence, 16(3):304-313, March 1994.

[35] H. Park and R. T. Chin. Decomposition of Arbitrarily Shaped Morphological Structuring Elements. IEEE Transactions on Pattern Analysis and Machine Intelligence, 17(1):2-15, January 1995.

[36] T. Pavlidis. Algorithms for Graphics and Image Processing. Computer Science Press, Rockville, Maryland, 1982.

[37] E. Rich and K. Knight. Artificial Intelligence. McGraw-Hill, USA, second edition, 1991.

[38] C. H. Richardson and R. Schafer. A Lower Bound for Structuring Element Decompositions. IEEE Transactions on Pattern Analysis and Machine Intelligence, 13(4):365-369, April 1991.

[39] C. Ronse. A Lattice-Theoretical Morphological View on Template Extraction in Images. Journal of Visual Communication and Image Representation, 7(3):273-295, 1996. 
[40] A. Schrijver. Theory of Linear and Integer Programming. Wiley-Interscience series in discrete mathematics. Jon Wiley \& Sons, 1986.

[41] J. Serra. Image Analysis and Mathematical Morphology. Academic Press, 1982.

[42] J. Serra. Image Analysis and Mathematical Morphology. Volume 2: Theoretical Advances. Academic Press, 1988.

[43] J. Serra and L. Vincent. An Overview of Morphological Filtering. Circuits Systems Signal Process, 11(1):47-108, 1992.

[44] P. Sussner and G. X. Ritter. Rank-based Decompositions of Morphological Templates. To appear in IEEE Transactions on IP (manuscript avaliable from authors).

[45] P. Sussner and G. X. Ritter. Decomposition of Gray-Scale Morphological Templates using the Rank Method. IEEE Transactions on Pattern Analysis and Machine Intelligence, 19(6):649-658, June 1997.

[46] T. A. Buja T. Hastie and R. Tibshirani. Penalized Discriminant Analysis. In Annals of Statistics, volume 23, pages 73-102, 1995.

[47] S. Takriti and P. Gader. Local Decomposition of Gray-Scale Morphological Templates. Journal of Mathematical Imaging and Vision, 2:39-50, 1992.

[48] M. Vanrell and J. Vitrià. Optimal $3 \times 3$ Decomposable Disks for Morphological Transformations. Image and Vision Computing, 15:845-854, 1997.

[49] J. Xu. Decomposition of Convex Polygonal Morphological Structuring Elements into Neighborhood Subsets. IEEE Transactions on Pattern Analysis and Machine Intelligence, 13(2):153-162, February 1991.

[50] H. Yang and S. Lee. Optimal Decomposition of Morphological Structuring Elements. In Proceedings of IEEE International Conference on Image Processing, volume 3, pages 1-4, Lausanne-Switzerland, 1996. IEEE Computer Society.

[51] X. Zhuang and R. Haralick. Morphological Structuring Element Decomposition. Computer Vision, Graphics, and Image Processing, 35:370-382, 1986. 


\section{Índice Remissivo}

$W$-operador, 27

SeqB $[A], 43$

$n$-ésima potência de um conjunto, 9

$n$-upla ordenada, 9

árvore de decomposição, 98

árvore de representação, 147

ínfimo, 15

SeqQ, 43

"branch and bound", 98

aberto de um conjunto, 18

abertura, 20

adição de Minkowski, 16, 25

algoritmo guloso, 56

análise de correspondência, 165

anti-simétrica

relação binária, 9

arestas de um EE, 48

base de um operador, 31

cardinalidade de um conjunto finito, 6

classe de equivalência, 10

coleção de extremidade direita fixa, 141

complemento, 15

composição de duas funções, 11

comprimento de uma decomposição, 42

concatenação de seqüências, 42

conjunto, 6

conjunto das partes, 6

conjunto de funções binárias, 26

conjunto finito, 6

conjunto infinito, 6

conjunto invariante, 18

conjunto parcialmente ordenado, 14

conjunto quociente, 10 conjunto vazio, 6

conjuntos disjuntos, 7

conjuntos iguais, 6

contradomínio de uma função, 11

coordenada coluna, 165

coordenada linha, 165

decomponível, 42

decomposição ótima, 42

decomposição de forma, 42

decomposição seqüencial, 42

decomposição seqüencial de um EE, 42

decomposição seqüencial de um conjunto, 42

dilatação, 28

disco euclidiano, 112

domínio de uma função, 11

dual-isomorfismo, 16

elemento estruturante, 28

elemento estruturante convexo, 39

elemento estruturante simplesmente conexo, 39,85

equivalência relação binária, 9

equivalência sob translação de conjuntos, 42

equivalência sob translação de intervalos, 124

erosão, 28

extremidade de um lado, 50

extremidade direita

intervalo, 21

extremidade esquerda

intervalo, 21

extremidade esquerda minimal, 144 
fechamento, 20

fecho convexo, 39

função, 11

função bijetora, 11

função composta, 11

função injetora, 11

função inversa, 11

função sobrejetora, 11

função teto, 11

granulometria de absorção, 34

grupo, 12

grupo abeliano, 12

imagem de uma função, 11

inf-decomposição de um operador, 31

inf-representação de um operador, 31

inteiro não negativo, 7

inteiro positivo, 7

intersecção de $n$ conjuntos, 7

intersecção de dois conjuntos, 7

intervalo, 21

intervalo maximal, 22

invariante de um conjunto, 18

invariante de um operador, 34

invariante por translação, 26

inversa de uma relação binária, 9

isomorfismo de reticulado, 16

kernel de um operador, 27

lado anterior, 49

lados de um EE, 48

limitante inferior, 14

limitante superior, 14

linguagem morfológica, 30

localmente definido, 27

máquina morfológica, 30

método guloso, 56

mapeamento, 11

Minkowski

adição, 16

subtração, 16

multiplicidade de um invariante, 42 nó invariante mais à esquerda, 108

nó viável, 100

núcleo de um operador, 27

nível de um nó, 100

observação, 165

operador, 19

operador abertura, 20

operador anti-extensivo, 19, 126

operador crescente, 19

operador de negação, 19

operador dual, 19

operador extensivo, 19

operador fechamento, 20

operador idempotente, 19

operador idempotente em um sentido estrito, 126

operador identidade, 19

operador inf-gerador, 29

operador propriamente limitado, 126

operador sup-gerador, 29

origem de $E, 16$

par ordenado, 8

poset, 14

poset dual, 16

próximo lado, 49

princípio da dualidade, 16

produto cartesiano de $n$ conjuntos, 9

produto cartesiano de dois conjuntos, 9

projeção ortogonal normalizada, 47

quadrado de um conjunto, 9

quadrado elementar, 36

reflexiva

relação binária, 9

relação binária anti-simétrica, 9

relação binária de equivalência, 9

relação binária inversa, 9

relação binária reflexiva, 9

relação binária simétrica, 9

relação binária transitiva, 9

relação de adjunção, 17 
relação de ordem, 14

relação ordenada, 9

representação compacta, 124

representação de um operador, 30

reticulado, 15

reticulado Booleano, 15

reticulado complementado, 15

reticulado completo, 15

reticulado distributivo, 15

reticulados dual-isomorfos, 16

reticulados isomorfos, 16

seqüência crescente, 64

seqüência crescente atual, 64

seqüência crescente atual maximal, 66

seqüência crescente maximal, 66

seqüência de nós mais à esquerda maximal, 108

seqüência de invariantes, 43

seqüência de nós mais à esquerda, 108

seqüência de subconjuntos, 41

seqüência decrescente, 63

seqüência decrescente atual, 63

seqüência decrescente atual maximal, 66

seqüência decrescente maximal, 66

seqüência elemento retangular, 69

seqüência gerada, 68

seqüência geradora, 68

seqüência possível, 102

seqüência restante, 63

seqüência retangular, 68

simétrica

relação binária, 9

solução parcial, 63

solução parcial atual, 63

sub-reticulado, 15

subconjunto, 6

subconjunto limitado, 14

subconjunto próprio, 6

subseqüência, 41

subseqüência de forma, 42

subseqüência de translação, 42

subtração de Minkowski, 16, 25 sucessão de $n-1$ adições de Minkowski, 36

sup-decomposição de um operador, 31 sup-representação de um operador, 31 superconjunto, 6

supremo, 14

transformação, 11

transitiva

relação binária, 9

transposto de um conjunto, 16

união de $n$ conjuntos, 7

união de dois conjuntos, 7

valor de uma função, 11

variável, 165

vetor projeção, 51

vetor retangular, 53 